\title{
Factors Affecting Reading for Pleasure Practices in Community Libraries in Thailand
}

By

Chommanaad Boonaree

A thesis submitted to the Victoria University of Wellington in fulfilment of the requirements for the degree of Doctor of Philosophy in Information Systems

Victoria University of Wellington 



\section{Abstract}

Studies have identified that reading for pleasure (RfP) has a positive influence on reading behaviour and is more important for academic success than family socioeconomic status (SES) (OECD, 2010). RfP can play a significant role in promoting literacy development, and reading behaviour in areas of low SES. In Thailand, however, RfP is a contested reading concept because reading is traditionally associated with academic purposes. To investigate the current status of RfP in Thailand and the role played by community libraries (CLs) in its promotion, a study was undertaken in the largest and most economically disadvantaged part of Thailand, the Northeast region, or Isan. This research lies within the social constructivism paradigm and uses an applied multiple case study design. The study has two objectives: 1 ) to identify the factors affecting RfP reading promotion practice in CLs in Isan, and 2) to determine how these factors affect RfP reading promotion practice in CLs in Isan. Data were collected in eight CLs by 1) semistructured interviews, 2) observations during library visits and library activity attendance, and 3) documentary analysis conducted on documentary sources. Qualitative data were analysed by open coding using NVivo11. The generation of a preliminary set of codes was deductively derived from literature and the research framework which was based on 1) Krashen's Free Voluntary Reading (FVR), and 2) Asselin \& Doiron's Ecological Framework for Community Library Developments.

Four theoretical categories were derived from the iterative inductive data analysis and the deductive literature review: 1) Thai Reading Context, 2) Thai Cultural and Social Factors, 3) Isan Socioeconomic Influences, and 4) CL Factors. They were used to write case reports on four types of $\mathrm{CL}: 1$ ) CLs established by an expert organization, 2) CLS under the Office of the Non-Formal and Informal Education (ONIE), 3) CLs established by health promotors, and 4) CLs established by individuals. This was followed by cross-case analysis derived from combining, comparing or contrasting the findings from each case, providing a higher level of interpretation showing in four themes: Theme A. Provision of Book Access in Disadvantaged Areas; Theme B. Literacy Development Support; Theme C. Provision of an Inclusive, Safe Atmosphere; and Theme D. Active Roles of Key Individuals in RfP. 
The study contributes to theory by redefining Duncan's conceptualisation of RfP which she asserts, "usually denotes an individual, silent activity" (2013, p. 5). People in a collective and oral society like to read together at CLs because the talk and socialisation bring joy and companionship. Control over book choice and a great variety of reading materials (Krashen, 2011; Asselin \& Doiron, 2013) were found to be crucial factors affecting RfP in both children and adult readers. However, an authoritarian approach by Thai adults frequently limits children's control over their reading choices. CLs in the study played an important role in enabling book access and literacy development support but they did not strategically position themselves as "central players in personal, social, cultural and economic improvement in their communities" (Asselin, Abebe \& Doiron, 2014, p. 5).

The study makes suggestions at the policy level relating to reading policy; event-based reading promotion strategies; the teacher-based learning style; lack of home-school partnerships; the knowledge-oriented mind-set; and the lack of reading to young children. A more strategic application of the Early Childhood Literacy Fund and the provision of better selected materials for CLs and school libraries would equalise access to children's books by those in marginalised communities. Reading Culture Promotion for Thai Learning Society Framework 2017-2020 needs to focus on intervening in the complex consignment system in the book market, identifying and developing reading leaders/volunteers, and raising awareness of RfP and media literacy. For LIS practice, understanding the impact of a low-anxiety atmosphere, informality and flat relationships in CLs could make library operations more relevant to the majority of Thai people who are often low on the socio-economic ladder but are generally eager to access books and read for pleasure. 


\section{Acknowledgements}

During my PhD journey, I received wonderful support, and advices from my supervisors, Professor Anne Goulding and Dr. Philip Calvert. Dr. Dan Dorner, who was the Research Degrees Programme Director in 2014 and my first secondary supervisor also greatly contributed to the smooth start of my study. Without their support, I would not have accomplished this endeavour.

My supportive parents, particularly my mother provides me endless love and resources, deserved the pride of the degree. I am also lucky to have friends in Wellington, who have always provided moral support, happy moments, and opportunities to explore more of this beautiful country. To name some, Jirayu Brennen, Wisetlak Wongpram-Jenkins, Pitchaya Aberg, Jo Ferguson, and Paul Cordue. You are my family. Sureepan Lemamnuay and Ngoc Ha Pham are my research companions who shared my ups and downs during this long journey, many thanks for their companionships.

I am greatly thankful to Victoria University of Wellington for granting me the VUW PhD Scholarship. Special thanks to administrative and academic staff members of the School of Information Management (SIM) for their strong support. Usha Varatharaju, Jean Grant, Dr. Janet Toland, and all others have been very supportive. All my PhD colleagues at SIM, made this journey enjoyable and possible by sharing their valuable experiences, and thoughts since I started my study. Thank you for your kind support.

Finally, all my research participants academically and practically widened my horizon on reading promotion. Their insightful and tireless contributions are invaluable for me and the country. 


\section{Table of Contents}

ABSTRACT

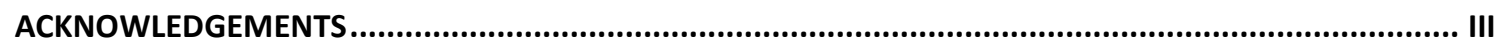

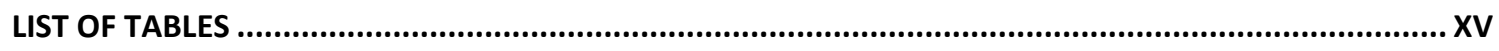

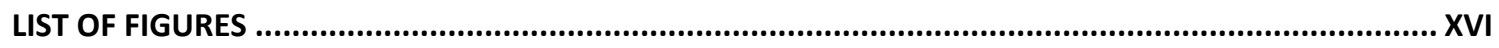

LIST OF ACRONYMS

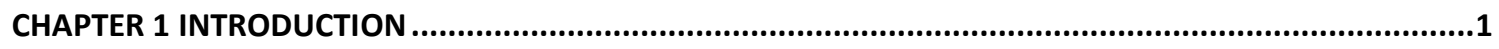

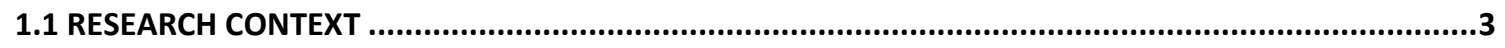

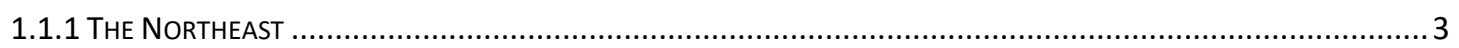

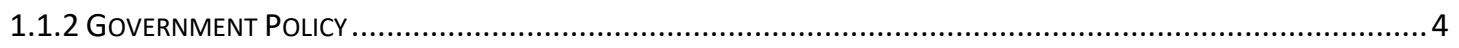

1.2 THE READING PROMOTION CONTEXT ..........................................................................................

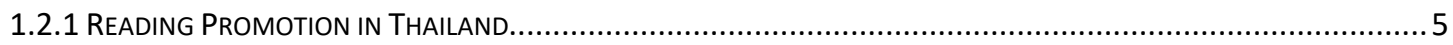

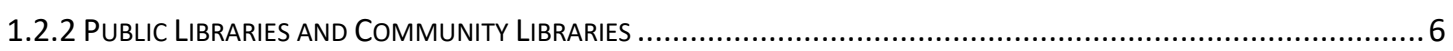

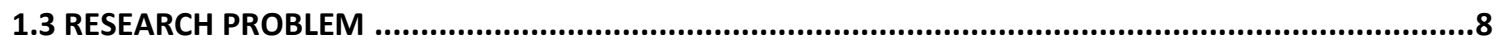

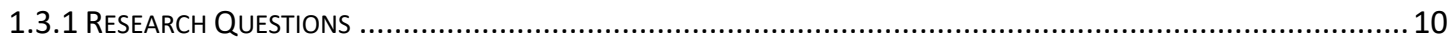

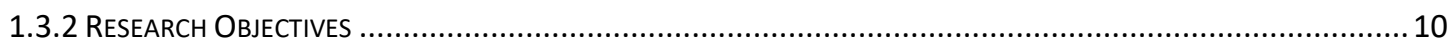

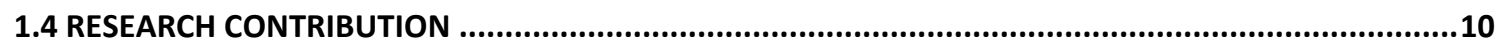

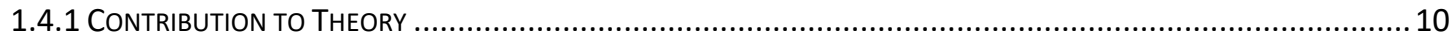

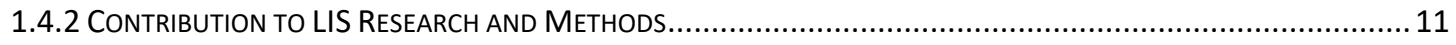

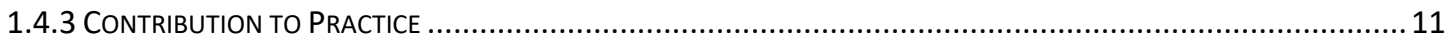

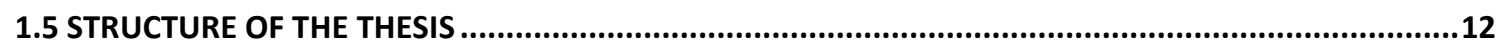

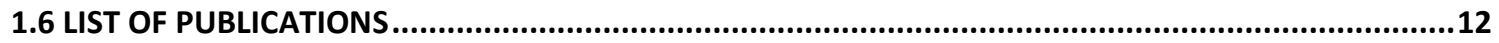

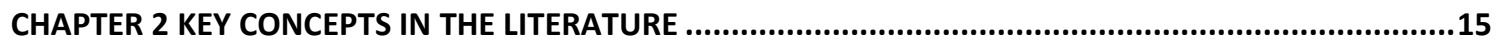

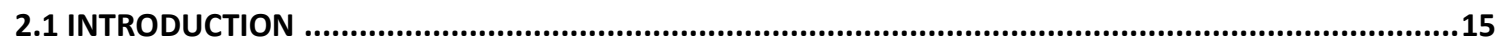

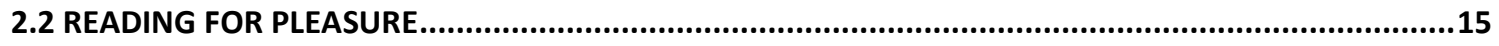

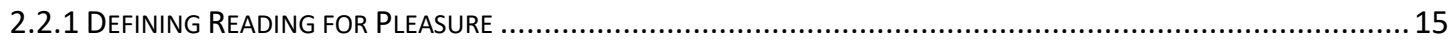

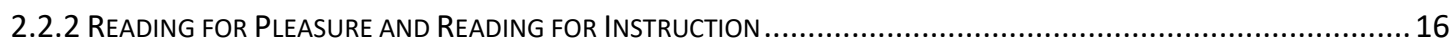

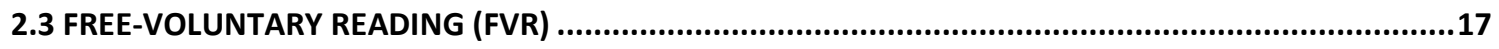

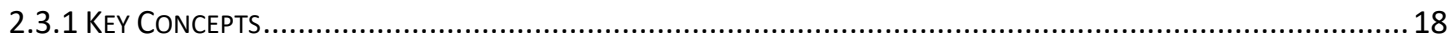

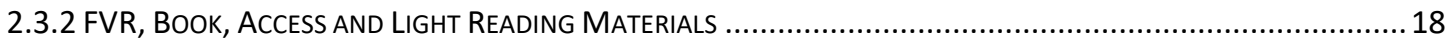




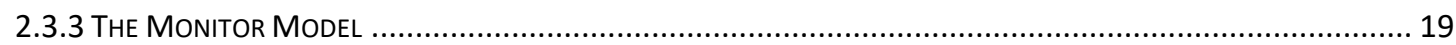

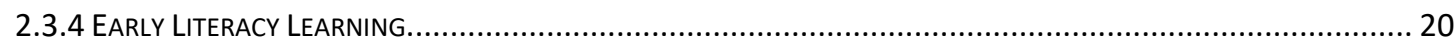

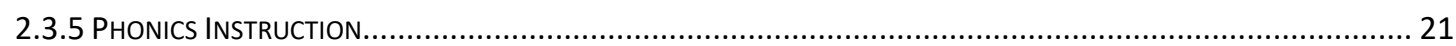

2.4 THE ECOLOGICAL FRAMEWORK FOR COMMUNITY LIBRARY DEVELOPMENT .............................. 21

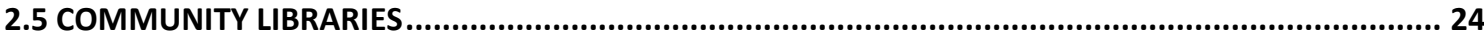

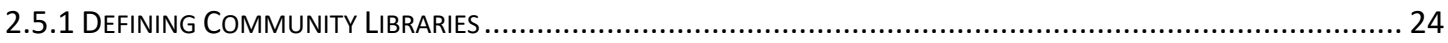

2.5.2 COMMUNITY LIBRARIES IN THE DEVELOPING WORLD .................................................................. 25

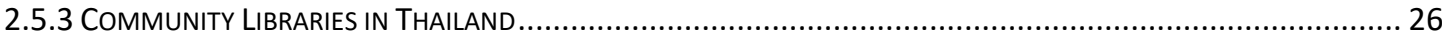

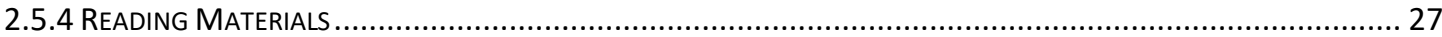

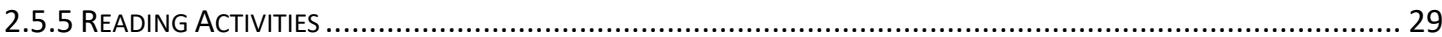

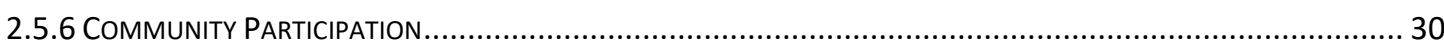

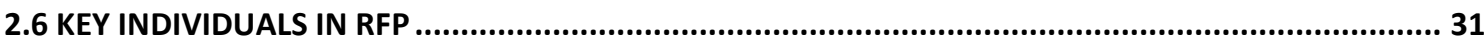

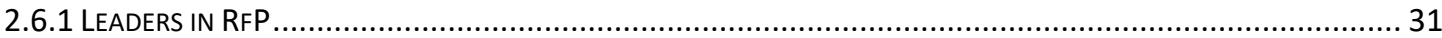

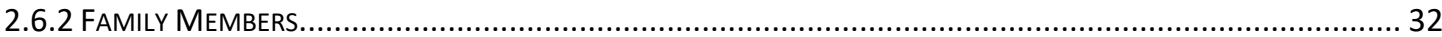

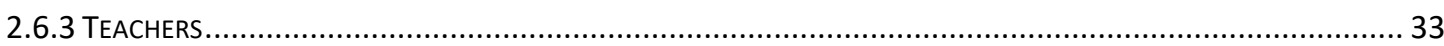

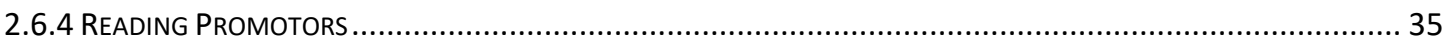

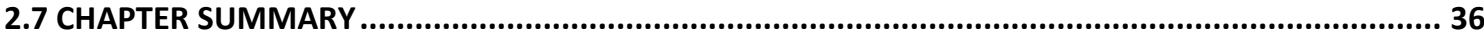

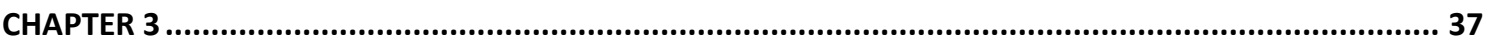

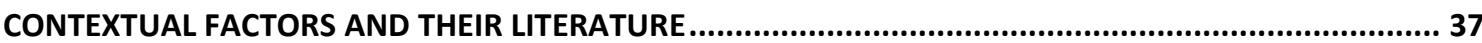

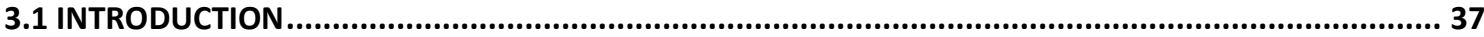

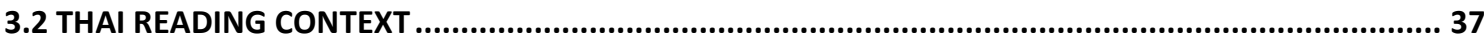

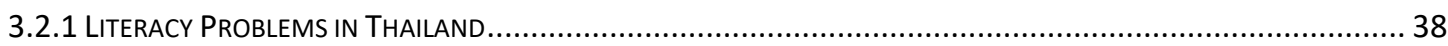

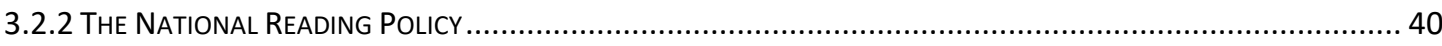

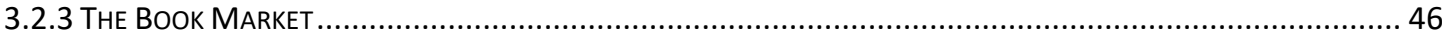

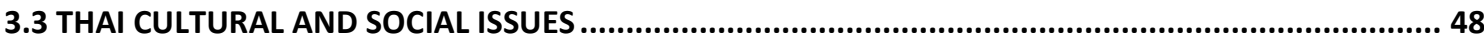

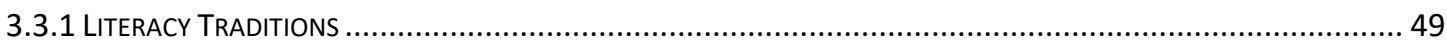

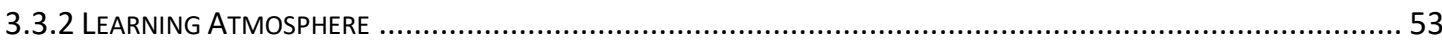

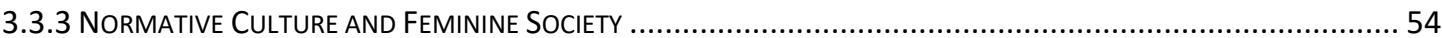

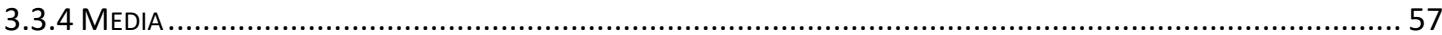

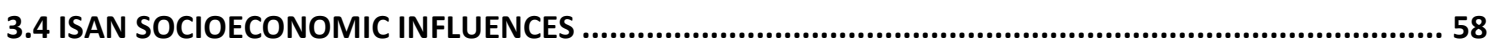

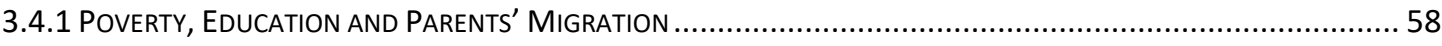

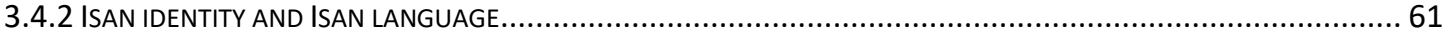




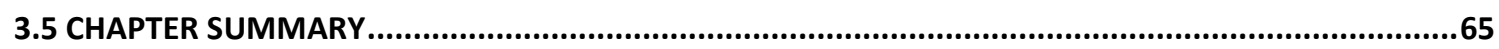

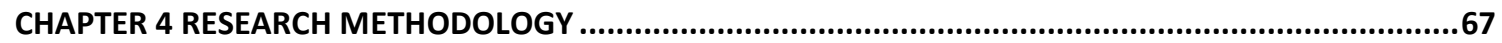

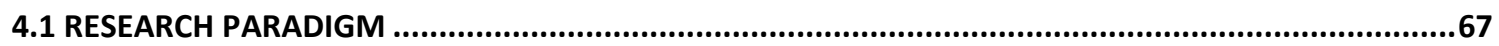

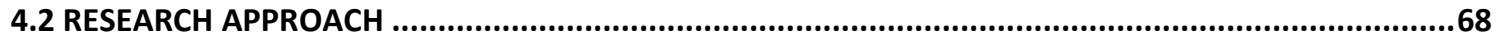

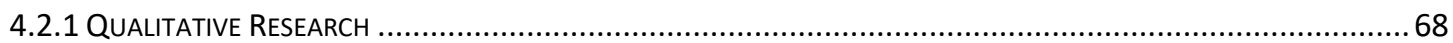

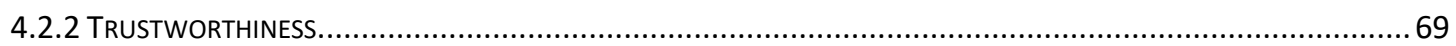

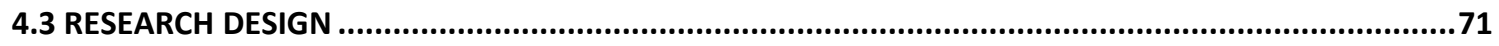

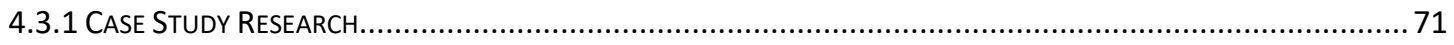

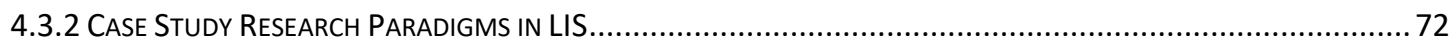

4.4 SELECTION OF RESEARCH SITES AND PARTICIPANTS ..........................................................73

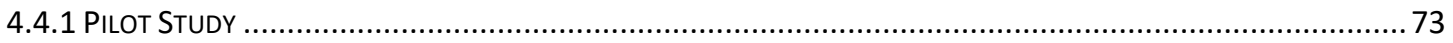

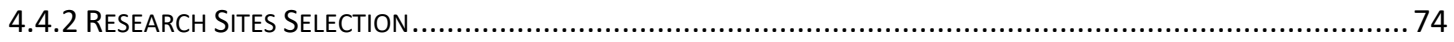

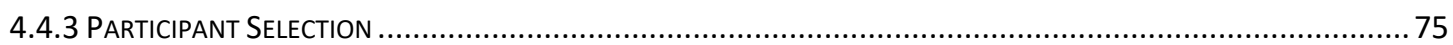

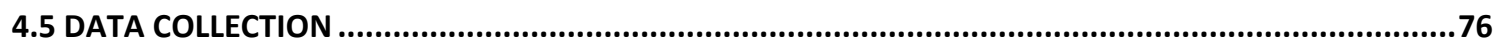

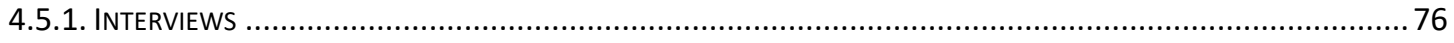

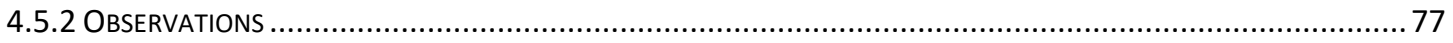

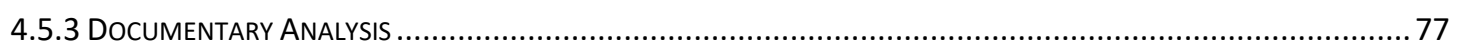

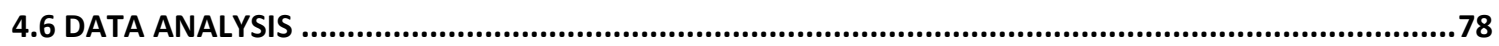

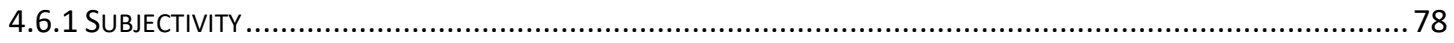

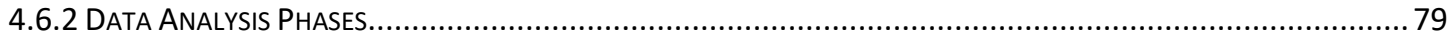

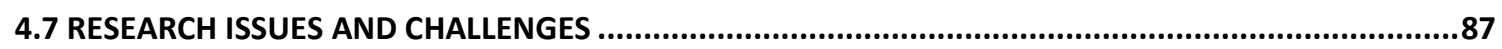

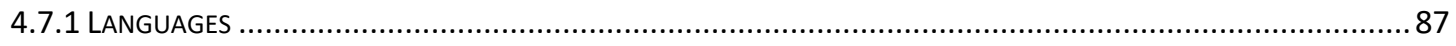

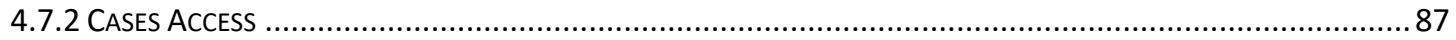

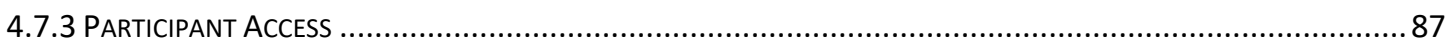

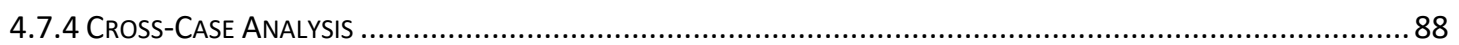

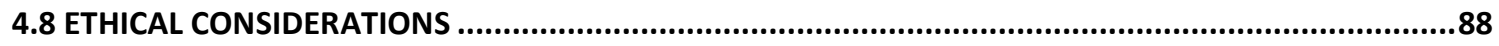

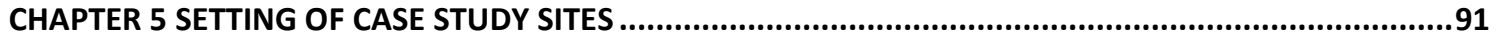

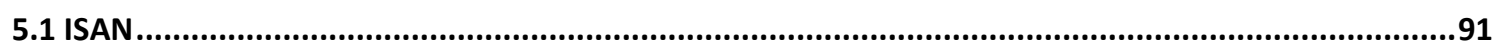

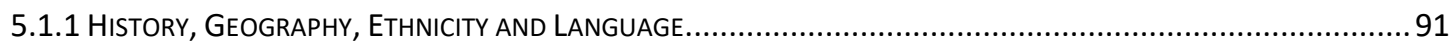

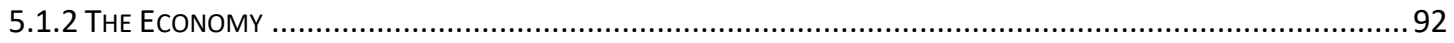

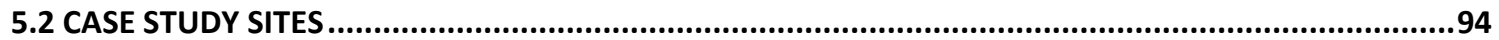


5.2.1 CASE StUdy 1: CLS ESTABLISHED BY AN EXPERT ORGANISATION ..................................................... 97

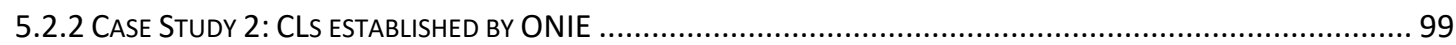

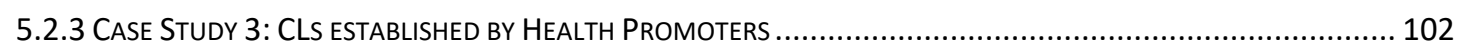

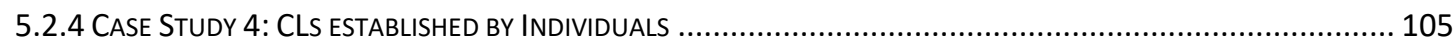

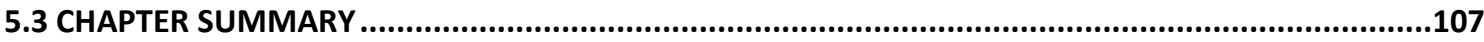

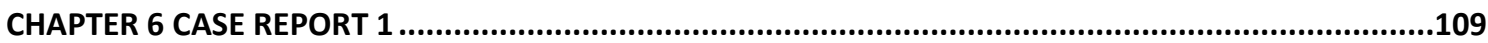

CLS ESTABLISHED BY AN EXPERT ORGANISATION ..............................................................109

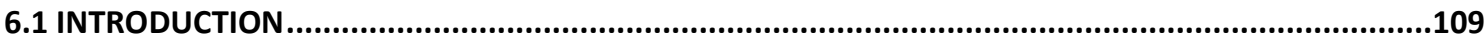

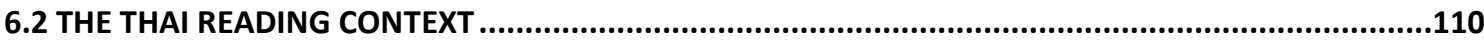

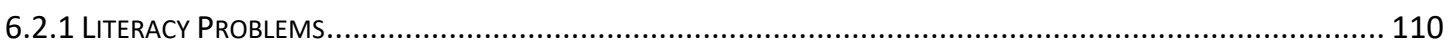

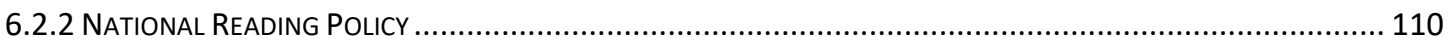

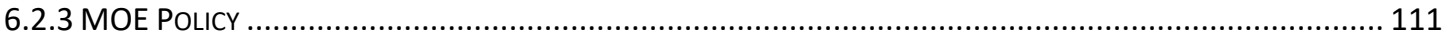

6.2.4 COORDINATION BETWEEN SUPPORTING ORGANISATIONS ............................................................... 112

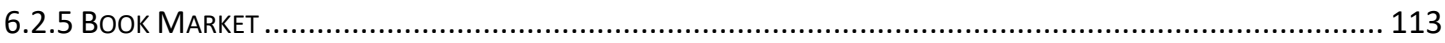

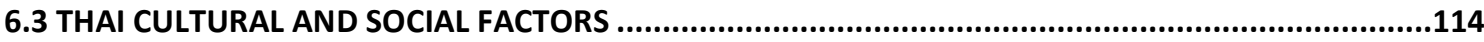

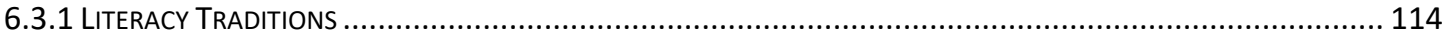

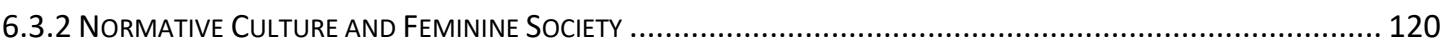

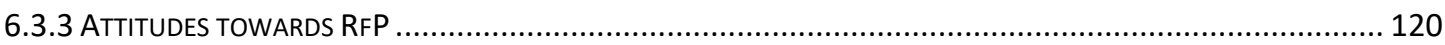

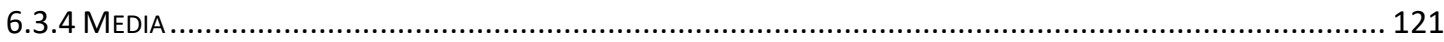

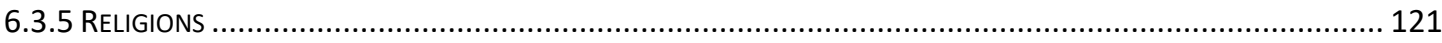

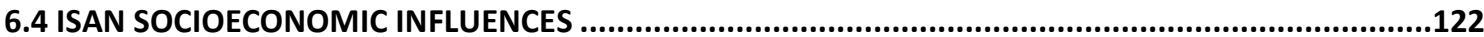

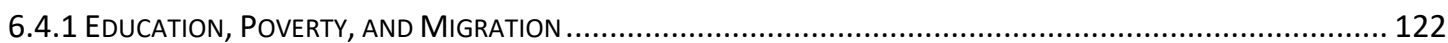

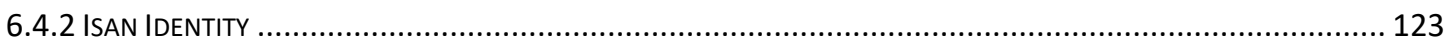

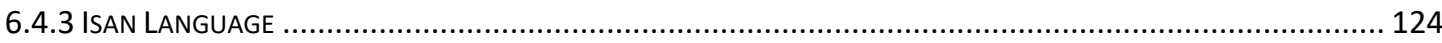

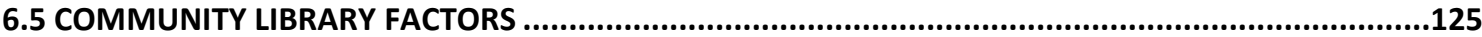

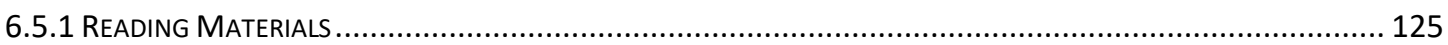

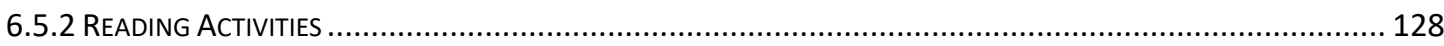

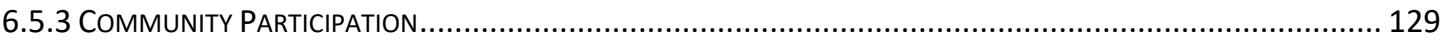

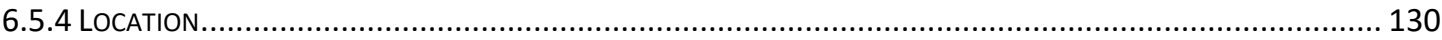

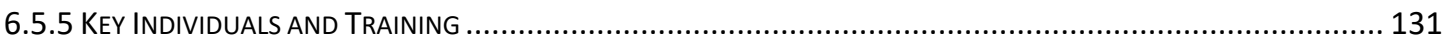

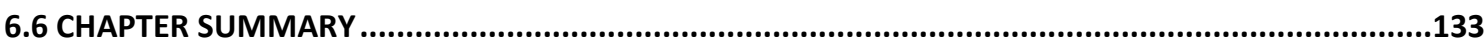

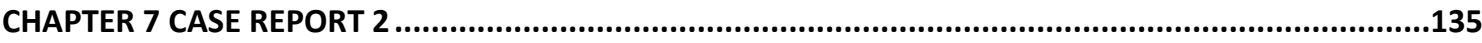

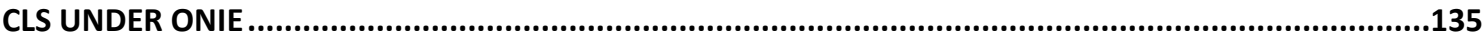




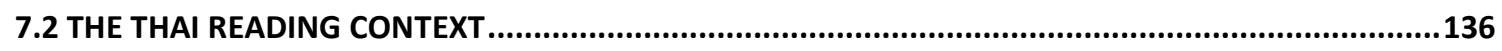

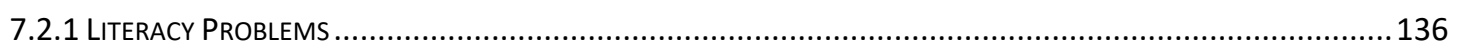

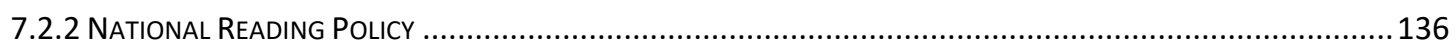

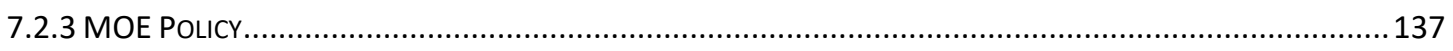

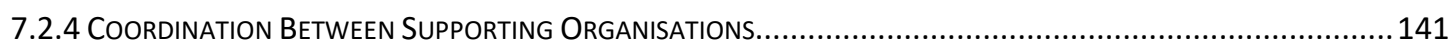

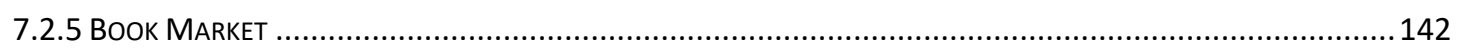

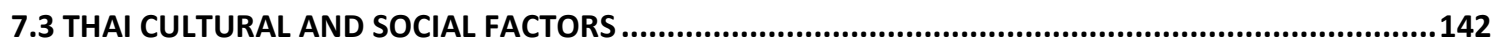

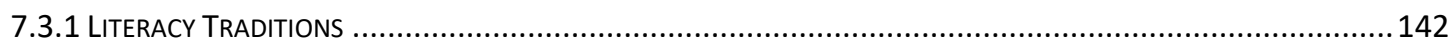

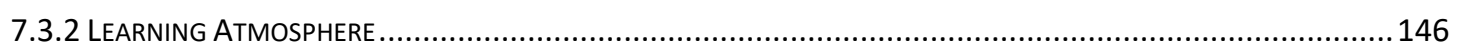

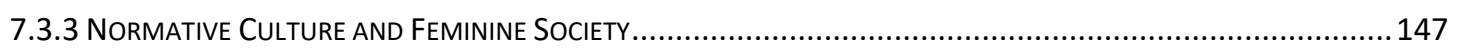

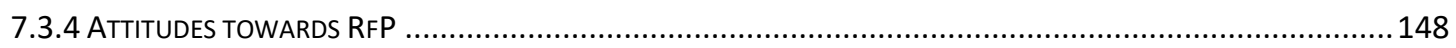

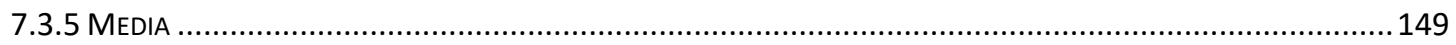

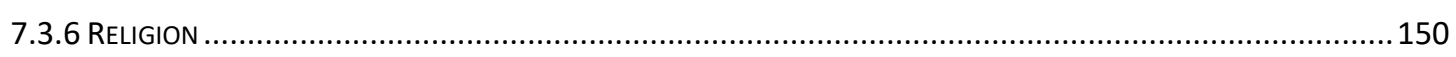

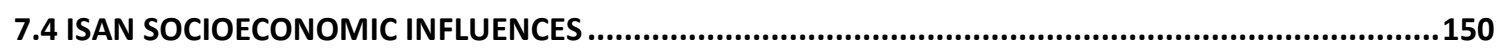

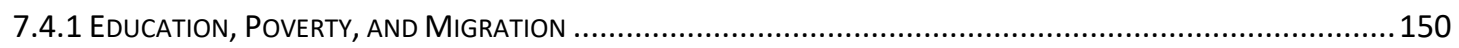

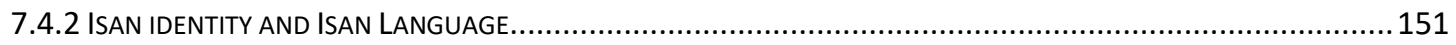

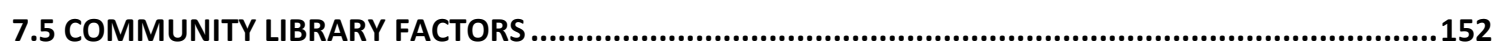

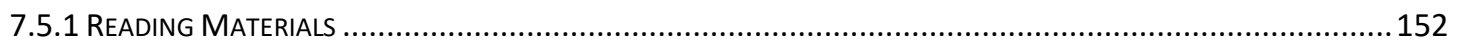

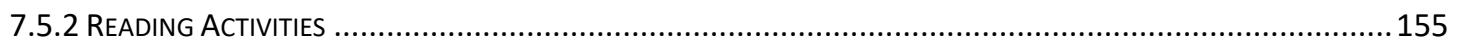

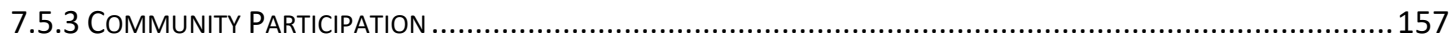

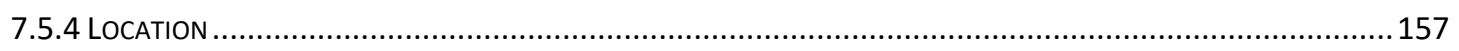

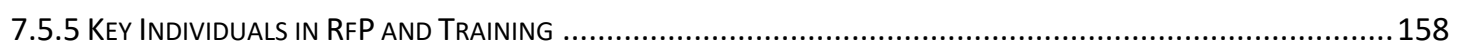

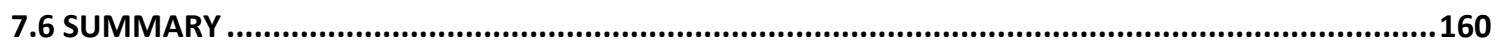

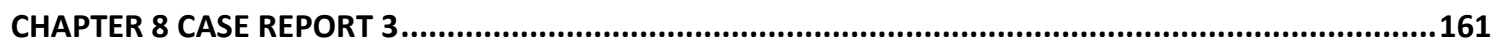

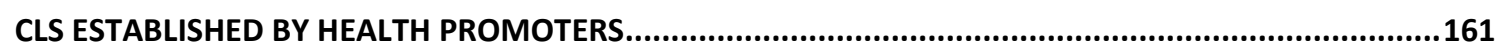

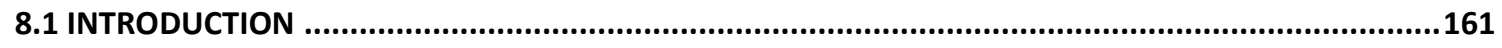

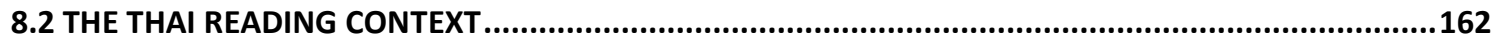

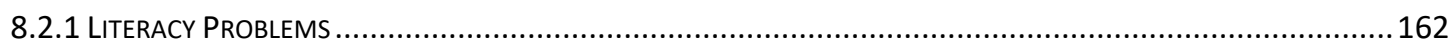

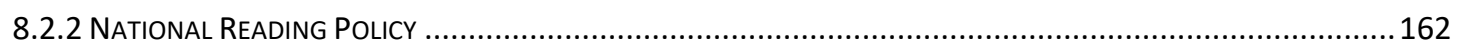

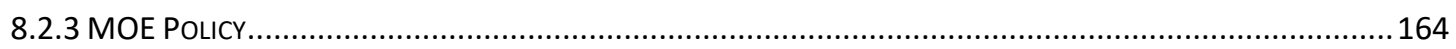

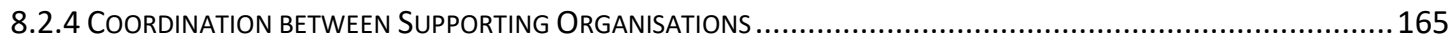

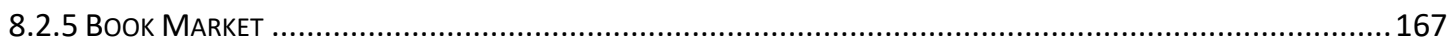




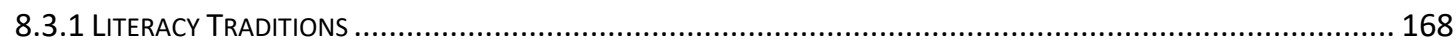

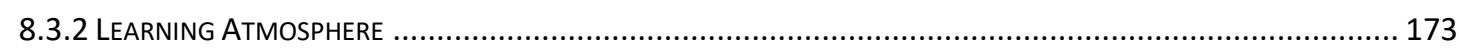

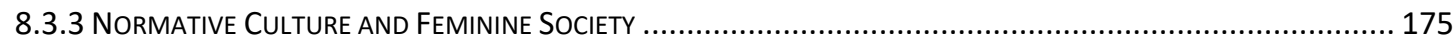

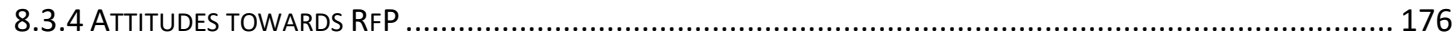

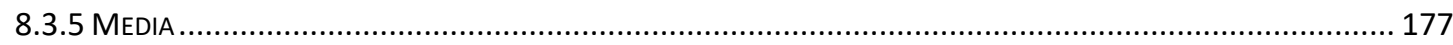

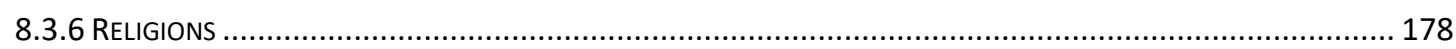

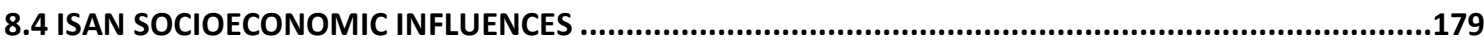

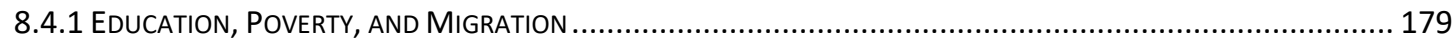

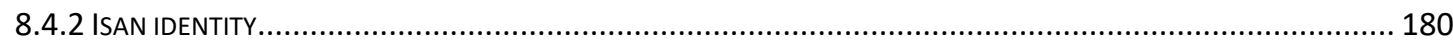

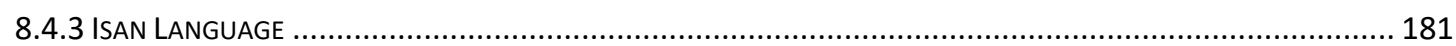

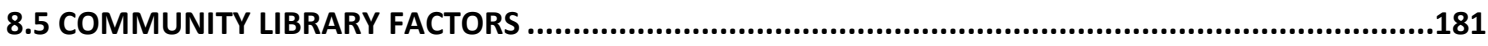

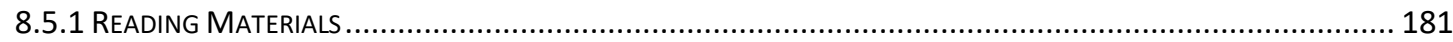

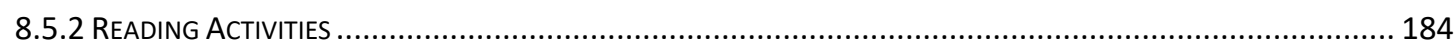

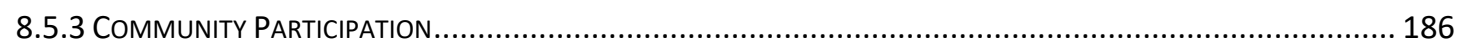

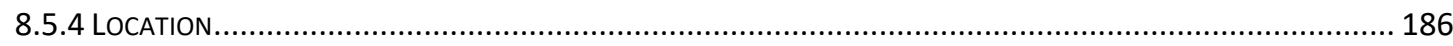

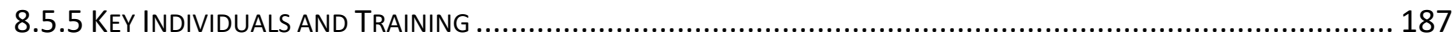

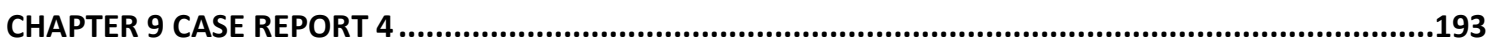

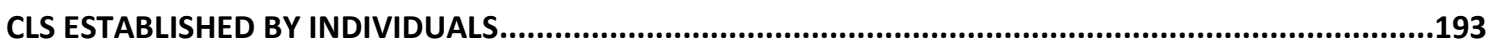

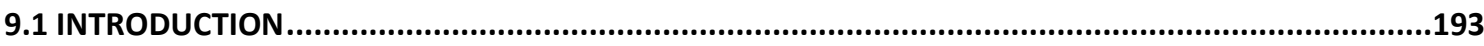

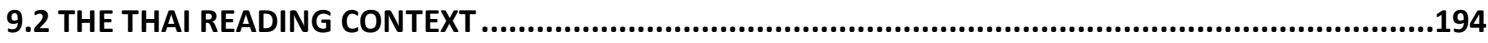

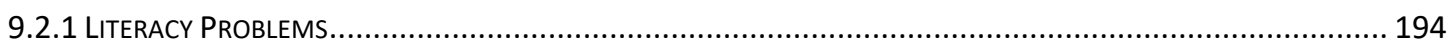

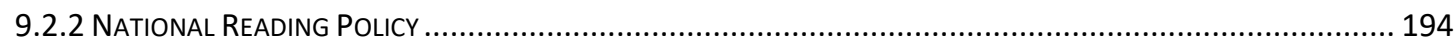

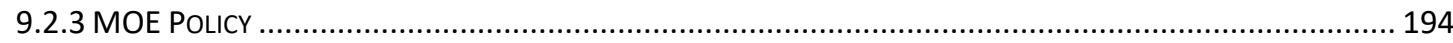

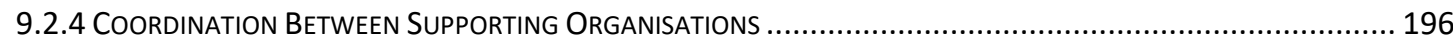

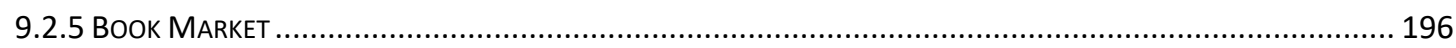

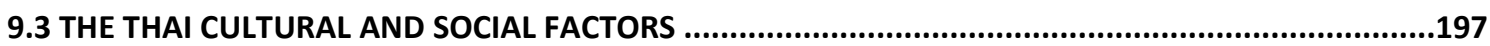

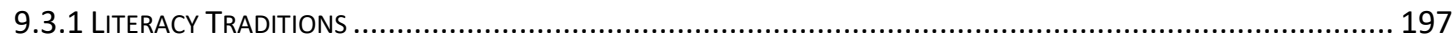

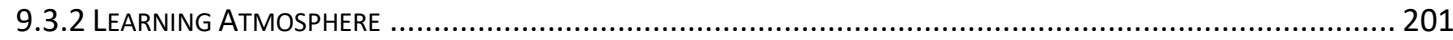

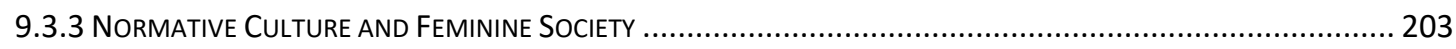

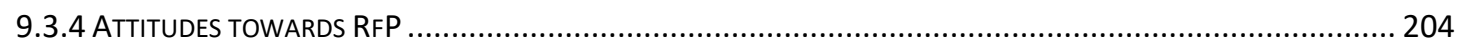

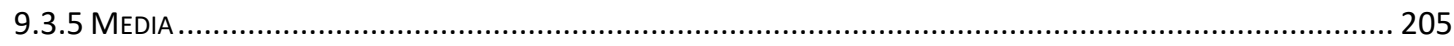

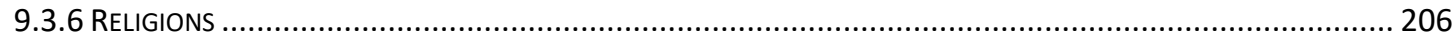




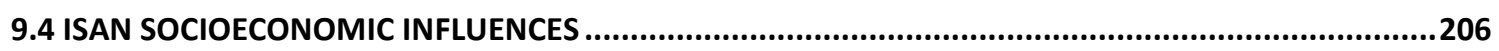

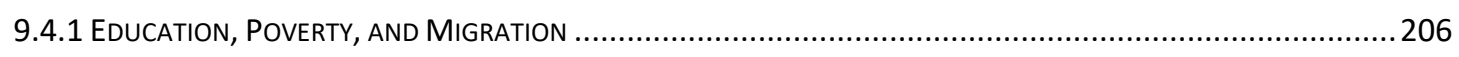

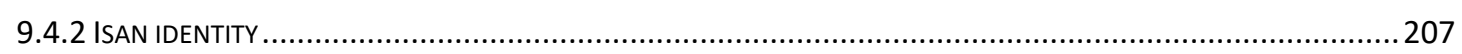

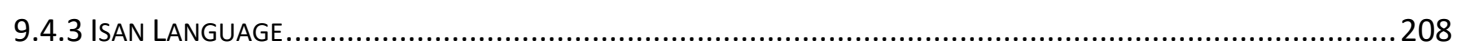

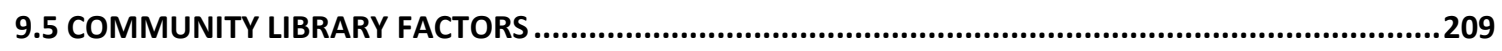

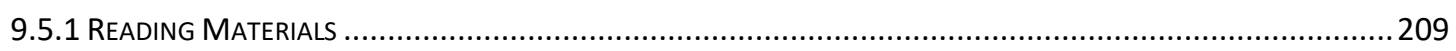

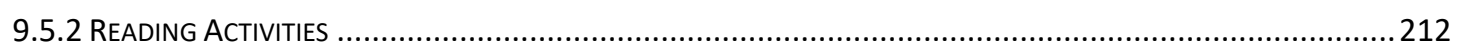

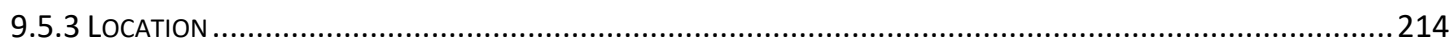

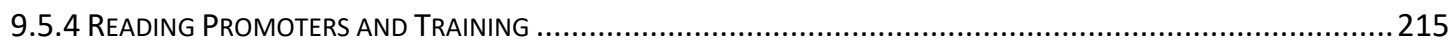

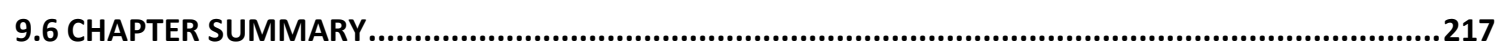

CHAPTER 10 FACTORS AFFECTING READING FOR PLEASURE IN COMMUNITY LIBRARIES...................219

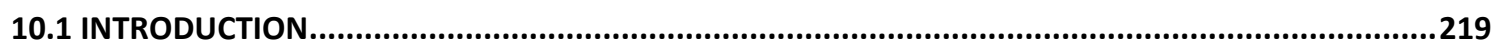

10.2 THEME A: PROVISION OF BOOK ACCESS IN DISADVANTAGED AREAS ....................................221

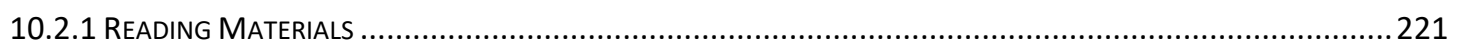

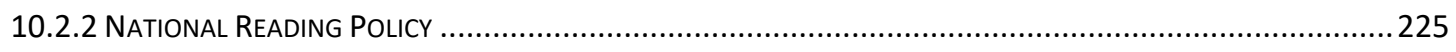

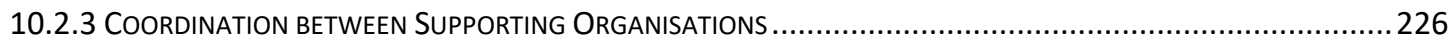

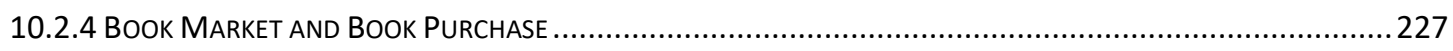

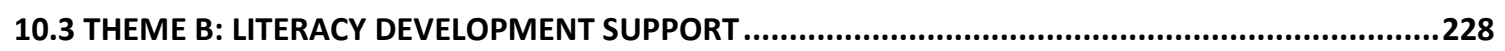

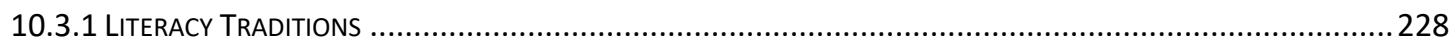

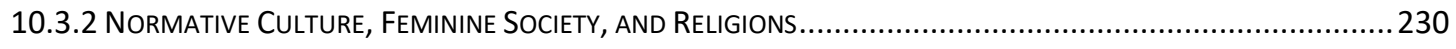

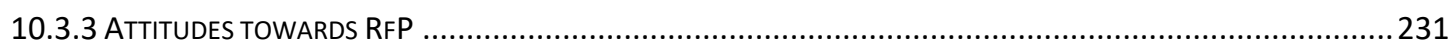

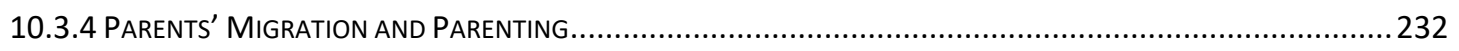

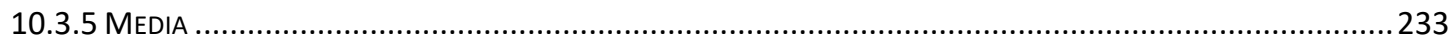

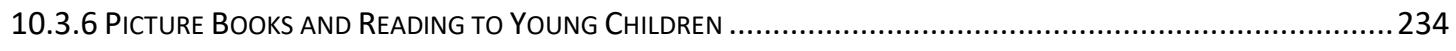

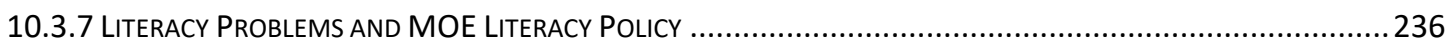

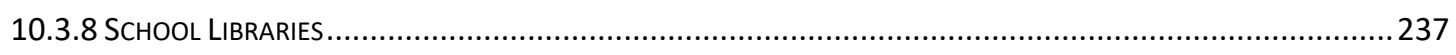

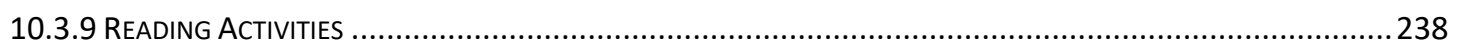

10.4 THEME C: PROVISION OF AN INCLUSIVE, RELAXED ATMOSPHERE.....................................242

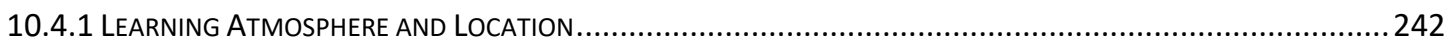

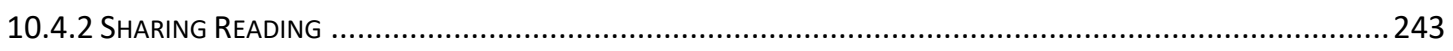

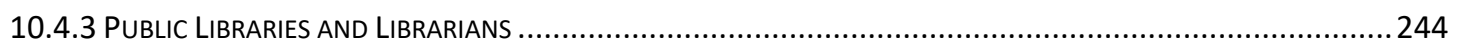

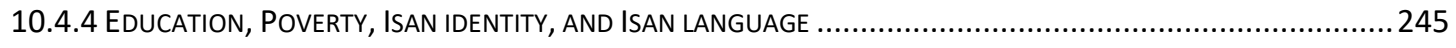

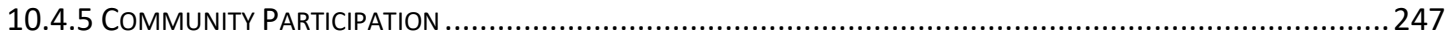




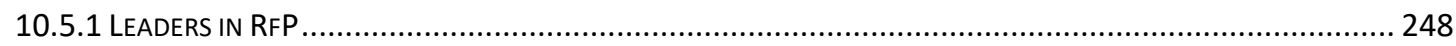

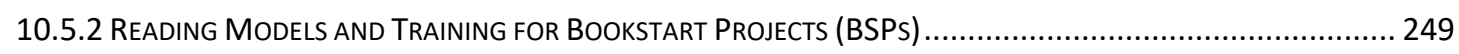

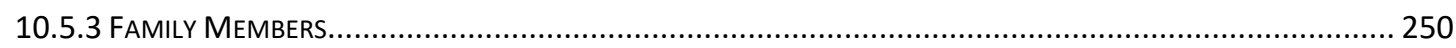

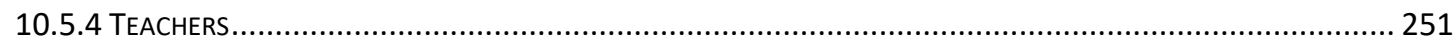

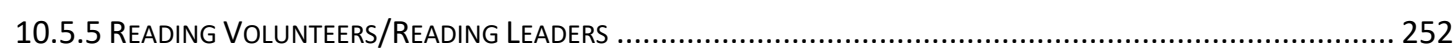

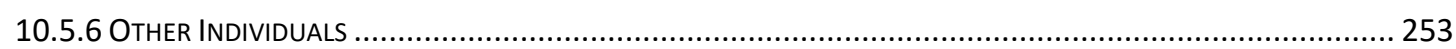

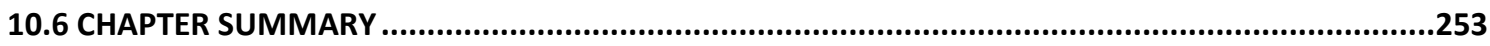

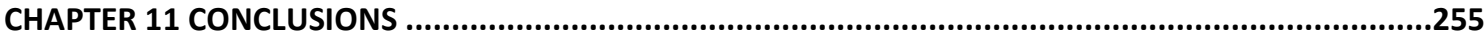

11.1 THEME A: PROVISION OF BOOK ACCESS IN DISADVANTAGED AREAS ....................................255

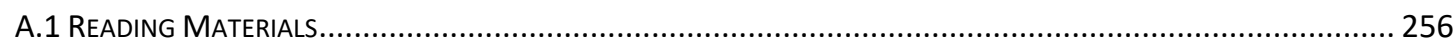

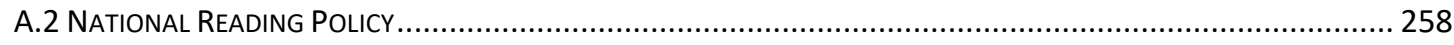

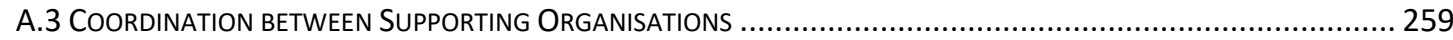

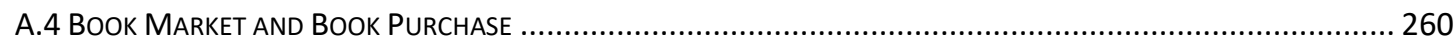

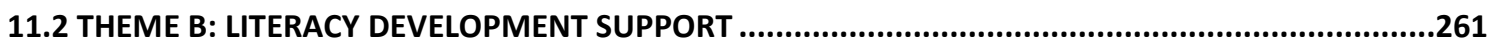

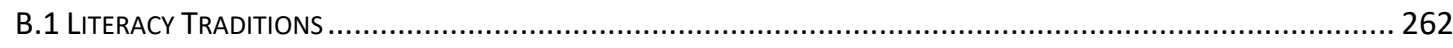

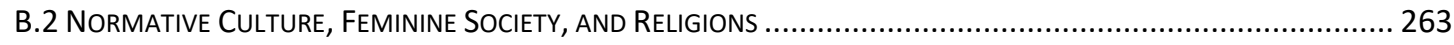

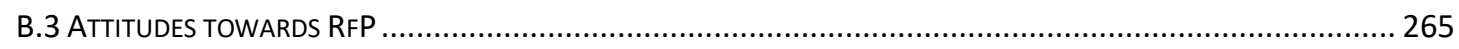

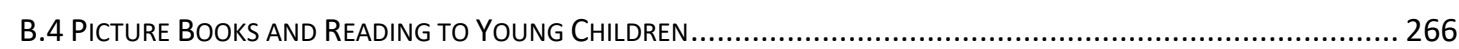

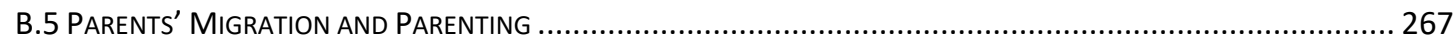

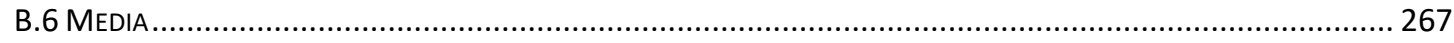

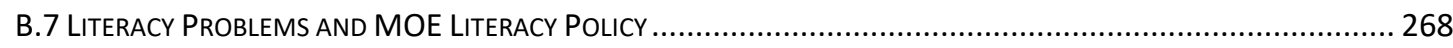

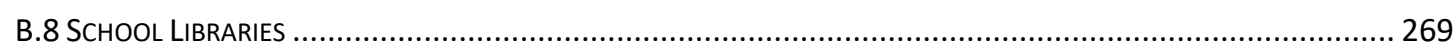

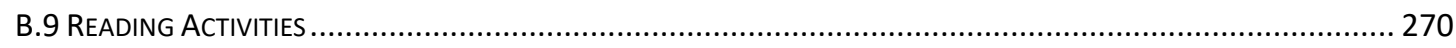

11.3 THEME C: PROVISION OF INCLUSIVE, SAFE ATMOSPHERE .....................................................272

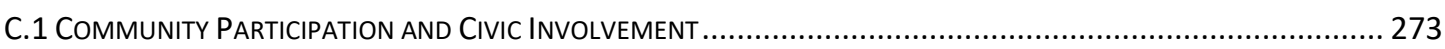

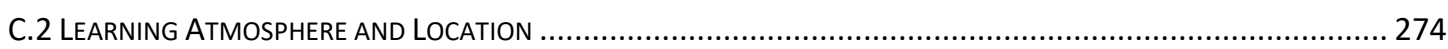

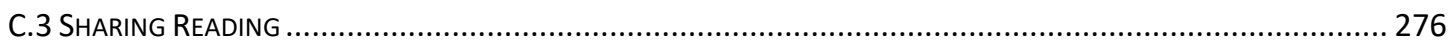

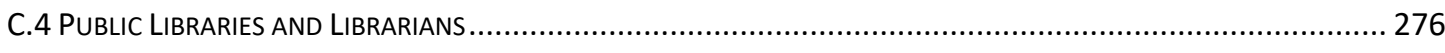

C.5 EdUCATION, POVERTY, ISAN IDENTITY, AND ISAN LANGUAGE ............................................................ 277

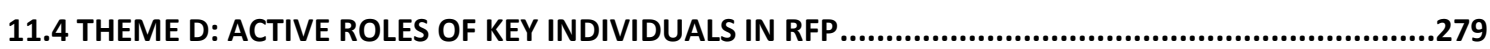

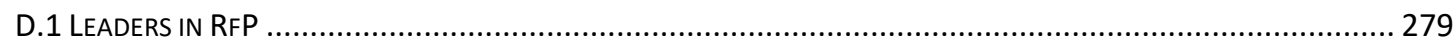

D.2 Reading ModelS ANd Training for Bookstart Projects (BSPS) ................................................. 280

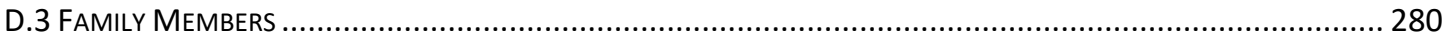




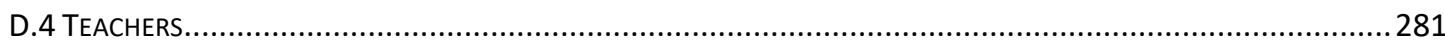

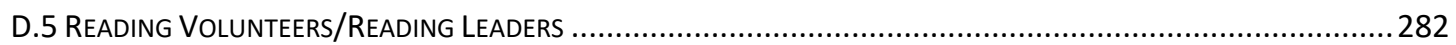

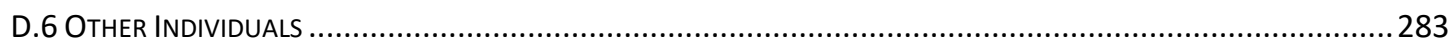

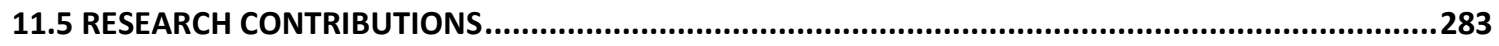

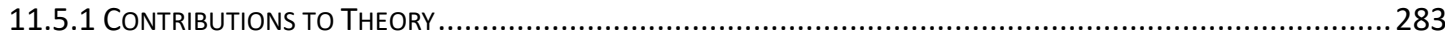

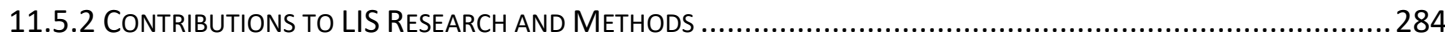

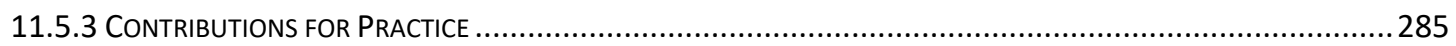

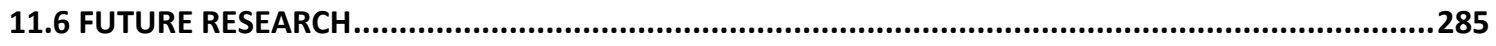

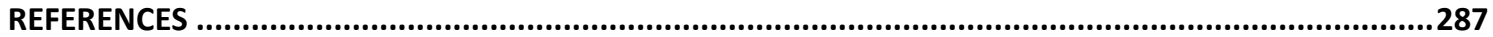

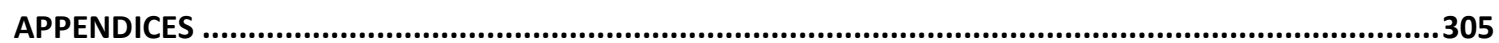




\section{List of Tables}

Table 2.1 Reading for pleasure and reading for instruction

Table 2.2 Mapping of community library management elements, Ecological Framework elements, and effective practices in reading promotion and literacy development elements

Table 3.1 Reading Culture Promotion for Thai Learning Society Framework 20172020

Table 3.2 Public expenditure on education per student by region, 2008 (Thai baht)

Table 3.3 Total students by region and level, 2008

Table 4.1 Summary of methodology elements

Table 4.2 Criteria and methods to ensure quality in qualitative research

Table 5.1 Regional GDP per capita in Thailand

Table 5.2 Details of case study sites

Table 5.3 Details of selected participants

Table 10.1 Themes and factors affecting RfP reading promotion practice in CLS 


\section{List of Figures}

Figure 2.1 Model of relationships of reading enjoyment, attitudes, behaviour and attainment

Figure 3.1 Proportion of functionally illiterate 15-year-olds in 2012 by type of location

Figure 3.2 The Reading System for Healthy Living by Happy Reading

Figure 3.3 Thailand minimum daily wage 2008-2018

Figure 3.4 Hofstede's National Culture Dimensions

Figure 4.1 Organisational categories

Figure 4.2 Substantive categories after data condensation and the first round of coding

Figure 4.3 Theoretical categories

Figure 4.4 Nvivo codes

Figure 4.5 Theoretical categories and theme

Figure 5.1 Map of Isan

Figure 5.2 GDP per capita and population data for the provinces in Isan in 2015

Figure 11.1 Relationship between factors in Theme A

Figure 11.2 Relationship between factors in Theme B

Figure 11.3 Relationship between factors in Theme C

Figure 11.4 Relationship of factors and themes in the study 


\section{List of Acronyms}

\begin{tabular}{|c|c|}
\hline BSP & bookstart project \\
\hline CDC & child development centre \\
\hline $\mathrm{CL}$ & community libraries \\
\hline CT & Central Thai (language) \\
\hline DPL & district public librarian \\
\hline ECD & Early Childhood Development \\
\hline ECLF & Early Childhood Literacy Fund \\
\hline FVR & Free Voluntary Reading \\
\hline \multirow[t]{2}{*}{ IFLA } & The International Federation of Library Associations and \\
\hline & Institutions \\
\hline MOC & Ministry of Culture \\
\hline MOE & Ministry of Education \\
\hline OBEC & The Office of the Basic Education Commission \\
\hline NGOs & Non-governmental organizations \\
\hline ONIE & Office of the Non-Formal and Informal Education \\
\hline $\mathrm{PHC}$ & public health centre \\
\hline PISA & The Programme for International Student Assessment \\
\hline PUBAT & The Publishers and Booksellers Association of Thailand \\
\hline $\mathrm{Rfl}$ & reading for instruction \\
\hline RfP & reading for pleasure \\
\hline SES & socioeconomic status \\
\hline $\mathrm{SBH}$ & Smart Book Houses \\
\hline SOT & sub-district ONIE teachers \\
\hline TAO & Tambon (Sub-District) Administration Organizations \\
\hline TK Park & Thailand Knowledge Park \\
\hline VHV & village health volunteer \\
\hline WA & Wicha Association \\
\hline
\end{tabular}





\section{CHAPTER 1 Introduction}

Over the decade of 1987-1996, driven by domestic and foreign investment, Thailand's economy was the fastest growing in the world (Warr, 2007). However, the World Bank (1996) and Asian Development Bank (Deolalikar, 2002) have discussed the negative effects of the robust economic success in Thailand suggesting that while it definitely reduced poverty, at the same time it increased income inequality.

Liamputtong (2014), the editor of Contemporary Socio-Cultural and Political Perspectives in Thailand, also noted that persisting inequalities alongside social and cultural changes in the country brought about new social movements and identities such as "the rise of new middle class and 'lower class' groups" (p. xv). The division between the two classes is wide and polarised; while most of the pro-government supporters (Red-Shirts) are poor farmers from rural Northeastern and Northern Thailand, the anti-government People's Democratic Reform Committee (PDRC) are middle class and high-class Thais (The Asia Foundation, 2013).

As a consequence of income inequality, the socio-economic status (SES) gap in society is significantly wide, particularly in Isan, the Northeast region of Thailand, the country's most economically and socially disadvantaged region (McCargo \& Hongladarom, 2004; Campbell, 2014). With one-third of the country's population, the region accounted for $40 \%$ of poor people in 2011 , the highest in the country. This is due to a large casual workforce and most occupations being clustered in the agriculture sector (Bird, Hattel, Sasaki \& Attapich, 2011).

Domestic migration of Isan people leading to acute social disintegration (Wasee, 2011) and economic disadvantage has resulted in educational disparities and a low SES (Fry \& $\mathrm{Bi}, 2013)$. The Reading of the Population Survey by the National Statistical Office (2012; $2014 ; 2016)$ also indicated that the region had the lowest reading rate/reading by young children under 6 years of age in the country in 2011, 2013, and 2015 (49.1, 53.5, and $55.9 \%$ respectively). 
Equity in educational opportunity is critical for the Thai economy; in 2015 the World Bank country director for Southeast Asia asserted that "The single most important thing for Thailand is to improve its education and skills outside Bangkok" (Changsorn, 2015). Successive Thai governments have been concerned about inequality in education and have initiated projects to cope with literacy problems since 1940 (Suwanpitak, 2008). However, literacy problems involving poor reading behaviour, functional illiteracy, and low reading attainment persist. Since reading behaviour and functional illiteracy are rather difficult to measure, the most readily available evidence demonstrating severe literacy problems is the reading attainment assessment by the Programme for International Student Assessment (PISA). The PISA 2015 results revealed Thailand had a ranking of 54th out of 70 OECD countries and that there had been a sharp drop in reading scores from 441 points in 2010 to 409 points in 2015. In the same test, Singapore ranked 1 st, and Vietnam ranked $8^{\text {th }}$. The detailed results showed the failure of Thai governments to allocate educational resources equally because the students in elite schools in Bangkok had very high scores and those in rural schools performed poorly. The distribution of scores was different from those in Singapore and Vietnam (Frederickson, 2016).

Moreover, the most recent UNESCO 2017/8 Global Education Monitoring Report identified that only $50 \%$ of Thai students who finished junior high school (age 15) "have a minimum proficiency level in reading" and there are 3.9 million illiterate adults in Thailand (UNESCO, 2017). There are 70 languages spoken in Thailand and the report highlighted that $50 \%$ of Thai students, ethnolinguistic minorities, are not educated in their mother-tongue languages.

It has been found that leisure reading or reading for pleasure (RfP) is an effective mechanism to improve reading ability and is more important for academic success than family SES (OECD, 2010; Sullivan \& Brown, 2013). It should be noted that RfP is crucial not only for children but also for emerging adult readers (Duncan 2013). Stephen D. Krashen, a prominent linguist who has promoted his theory on RfP called Free Voluntary Reading (FVR) for more than 20 years, insists that access to printed materials through school and public libraries and FVR are two highly effective approaches to enhance literacy development amongst children in poverty (1993; 2011a; 2013). 
Adkins and Brendler (2015) also suggested that library material provision and fostering a literate environment and freedom in reading choices, are crucial factors underpinning reading motivation in teenagers. The universal findings conform with Krashen's observation that:

The frequent rationale for not funding libraries is the complaint that "giving them books won't help; they won't read anyway." There are two responses to this complaint. The first is that if there are no books, it is certain that no reading will take place. The second is that "they won't read" accusation is wrong. It is incorrect for English reading and reading in other languages. Given access to books, young people read (Krashen, 2011, p.28). Therefore, book access via library collection and RfP can play significant roles in literacy development for people in disadvantaged areas. This study seeks to understand the phenomenon of reading for pleasure in community libraries, a new type of library provision (see 2.5), in Northeast Thailand. The following sections present the research context of this specific disadvantaged part of Thailand and the reading promotion context in Thailand. The research problem, the research questions and objectives, and the research contributions are then provided in the remainder of the chapter.

\subsection{Research Context}

As noted above, over the past 30 years, Thailand has enjoyed remarkably successful economic development. However, the negative impact of development, income inequality, has been an undesirable side effect (Warr, 2007). The impact of wealth inequality can be observed perhaps most acutely in the Northeast region of Thailand.

\subsubsection{The Northeast}

Thailand can be roughly divided into four distinct regions according to geography and ethnicity: the North, the Northeast, the Central region and the South. Historically, each region has its own language (both written and spoken) and religious practice (different Buddhist practices), resulting in a distinctive culture (Keyes, 1989). However, to avoid European expansion in the late 19th century, there was a drive to homogenise ancient Siam and create a modern nation state. Wyatt $(1969$, p. 379) stated that "If the Thai did not reform and innovate, they knew that they stood little chance of maintaining their 
independence and their identity." Therefore, persistent efforts to establish national ideology, administer the outlying areas of Siam, and standardise various aspects of Siam's social and economic life were initiated by King Chulalongkorn the Great (Rama V, 1853-1910) (Keyes, 1989).

In 1892, the Lao-speaking area, the domain of Siam in the northeast (Isan) was integrated into Siam (Keyes, 1989). It resulted in a loss of autonomy for Isan's cities and in 1871 the Isan written language used in traditional education in temples, was legally banned (Tambiah, 1968). However, at present, around 20 million Northeasterners, the largest minority group in Thailand, still speak Lao Isan as a vernacular language (Draper, 2010). Therefore, they learn Central Thai (CT) at school as a second language. As a result, they have become a literacy disadvantaged group. Fry and Bi (2013) noted that people in the region have the lowest access to pre-school in Thailand (ages 3-5), have the highest percentage of mid-year school dropouts, have the lowest subsidy per head per student, and have the lowest national test scores.

Education reforms of the late 19th century have had a significantly negative impact on the Thai education system. Fry and Bi (2013) stated in their study that the reforms yielded the unexpected result of over-centralisation and a related consequence has been that the education system pays little attention to local needs and has a disregard for cultural democracy.

The disregard of this ethnolinguistic minority, the Isan, leads to the continuing disempowerment and low self-esteem of Isan people. Choices of library access of rural and urban people may not only be due to the proximity of facilities but also the inferiority complex of rural Isan people (Draper, 2010; Myers, 2005).

\subsubsection{Government Policy}

Decentralised policy implementation has recently been the predominant approach of successive Thai governments. However, it has encountered a range of difficulties in its practical application. Possible impediments hindering Thailand's development are vertical social structures that favour the advantaged classes (Wasee, 2014). Cuesta and Madrigal (2014) found that despite a decentralised education policy, education expenditure amongst regions in the country is not "pro-poor" but rather "pro-rich"; thus, 
"the current decentralised allocation of educational spending is not consistent with an equity-enhancing goal" (p. 239). The outcome of this is that the per student public expenditure on education in the Northeast lags behind other regions, especially Bangkok (Tables 3.2 and 3.3).

In conclusion, the wide gap between the new middle class and the lower class (mostly Isan people) in Thai society has been identified by a number of contemporary studies. This is particularly pronounced in the Northeast region which suffers from economic and academic disparities. To help develop the quality of education (which in turn will help address socioeconomic inequality), a major issue of concern is literacy development. One community-based element with the role/potential to support literacy development throughout the country is the community library.

\subsection{The Reading Promotion Context}

\subsubsection{Reading Promotion in Thailand}

In Thailand, there is no central authority responsible for reading promotion in the country and, until 2017, there was no national reading policy. The library system and its activities to support literacy and reading are, therefore, fragmented and limited. The major reading initiatives in Thailand led by government agencies have been ineffective, especially those involving primary school libraries and public libraries which operate under two separate offices in the Ministry of Education (MOE). The ineffectiveness, which is reportedly caused by personnel and budget issues, have been ignored for a long time (Pradit, 2006; Sacchanand, Prommaphan, \& Sacchanand, 2008).

In contrast, the private sector is evidently more active. Cooperation between the Publishers and Booksellers Association of Thailand (PUBAT) and 32 organisations in 2007 successfully advocated for reading to be promoted as a government national agenda. This resulted in "the Decade of Reading 2009-2018" campaign (Na Mahachai \& Thoopkrajae, 2007).

Independent organisations have been established by the government to promote reading, as an alternative to bureaucratic ministries. The first was Thailand Knowledge Park (TK Park). While TK Park focuses on urban areas, the second organisation, Reading 
Culture Promotion Programme (Happy Reading), aims to build links between reading promotion organisations and to promote a reading culture among Thais in deprived areas (Happy Reading, 2015).

In 2017, the first national reading policy was announced by the Ministry of Culture (MOC). However, the fragmentation of responsibility for reading promotion under the complex ministerial administrations persist. The policy manual (Ministry of Culture, Ministry of Education, and Ministry of Interior, 2017) lists 31 major organisations involved with reading promotion in Thailand. This number includes only the lead organisations; in detail it lists five organisations under the MOC, five organisations under the MOE, 10 organisations under the Ministry of Interior, and three organisations under the Ministry of Public Health (MOPH) as being involved with reading promotion. Although MOC, TK Park, and Happy Reading work as linking agencies between these organisations, these three agencies do not have legal authority, nor the budget to mobilise projects in each organisation listed. As of 2018, the practical application of the policy relies upon cooperation from organisations who may see themselves as informally or accidentally involved with the MOC's reading culture promotion initiative. This is different from the streamlined reading promotion activity in Singapore, for example, where the National Library Board administers all aspects of reading promotion in the country.

\subsubsection{Public Libraries and Community Libraries}

Public libraries in Thailand are operated by the Office of Non-Formal and Informal Education (ONIE) under the MOE (ONIE, 2014). However, due to severe problems arising from insufficient budgets and staff, public libraries have failed to meet the minimum standards expected of a modern public library service (Sacchanand, Prommaphan \& Sacchanand, 2006). Additional efforts have been made to extend the services into rural areas in the form of mobile libraries. Methods used to deliver books to people who live in different parts of the country include elephants, boats, and book vans (Butdisuwan, 2000). Cheunwattana (2003) provided the example of portable or mobile libraries projects established in the 1980s by universities aimed at children in rural areas. 
In 1972, the Office of the Non-Formal Education Commission (the former name of ONIE) established village reading centres/corners to increase the literacy rate among the rural Thai population. These were mainly aimed at school leavers and adults. The vast number established by $1999(35,514)$ made it one of the most important reading resources for rural people (Cheunwattana, 1999).

Stranger (2014, p.93) defined a CL in developing countries as "a library, or a library-like collection and lending service, that is not part of a public library program and is run by one or more people from the community it serves." These libraries are diverse in nature and were started in response to people's needs in deprived areas (Asselin, \& Doiron, 2013; Hog, 2014). In Thailand, Changket (2011), identified eight types of CL initiatives in the country. However, her definition of $\mathrm{CL}$ covers libraries under public library programmes supported by local government, therefore falling outside the definition given by Stranger above.

Most CLs in Thailand originated as village reading centres, initiated by the government and operated by ONIE. Despite the fact that the centres have not functioned well (Cheunwattana, 1999), the national reading survey in 2009 (National Statistical Office, 2010) revealed that Thais in both urban and rural areas ranked village/community libraries as one of the top three most effective strategies to encourage reading behaviour. Moreover, a study by TK Park (2010) indicates that people with primary education, and those who work in the agricultural sector use the village/community library the most.

In 2013, ONIE initiated the newest CL project, the Smart Book Houses Project, as a part of the national reading agenda. However, the uncomfortable fact discovered in Malaysia (Abu, 2014), Indonesia (Håklev, 2010) and other developing countries (Hog, 2014) is that top-down government library models are not viable and sustainable. Thus, additional studies need to be undertaken to seek ways to support various types of $\mathrm{CL}$, as it is the newest type of library in the developing world (Chisita, \& Chiparausha, 2017) and proven to be an effective way to increase literacy rates and encourage reading behaviour among rural people (Ahmed, 2010; READ Global, 2015; Shrestha \& Krolak, 2015). 


\subsection{Research Problem}

The problem this research seeks to address is that little is known about how CLs in Thailand support people's reading for pleasure practices. A clearer understanding of the factors impacting on CLs capacity to support RfP is needed for the development of appropriate policy and practice to help improve reading behaviour and reading attainment in the country. Studies in Thailand have indicated that although Thai people generally highly value the ability to read, a lack of reading enjoyment results in Thais reading less than people in other south east Asian countries, such as Singapore, Vietnam, Japan and Korea (TK Park, 2008; 2010). This reading behaviour problem is more pronounced in disadvantaged families (both rural and urban-poor areas), where people cannot afford books (National Statistical Office, 2014).

Village/community libraries are crucial for disadvantaged people in Thailand for several reasons. First, the National Economic and Social Development Board (2011, p.103) identified that "the creation of educational opportunity for the majority of Thai people is in non-formal education". Thus, public and community libraries can serve more people than school or university libraries. Second, there is a clear division of library choices, with the less educated choosing community libraries and those with more extensive education opting for public libraries (TK Park, 2010). Third, Makut Ornrudee, a crusader for reading, persistently argues that in the Thai context, reading encouragement cannot fully be the responsibility of families, because the majority of Thai families live in poverty. He insists that quality book access via school libraries and CLs are keys to the love of reading. His $\mathrm{CL}$ projects in a southern province showed that rural people are eager to read if a variety of quality books is provided (Kongrut, 2012). This book access principle supports Krashen's FVR principle on literacy development by libraries for children in poverty (Krashen, 2011; 2013).

RfP practices in CLs have the potential to be an effective mechanism to enhance reading behaviour, leading to stronger literacy skills and educational accomplishment, thus helping address problems associated with poverty in disadvantaged Isan. The RfP concept has been widely accepted in the west as a way of encouraging increased reading behaviour (Save the Children Fund, 2014; Carnoy, \& Rothstein, 2013). In New Zealand, 
for example, the National Library of New Zealand (2017) proposed in its strategic directions to 2030 that: "Our aspiration is for a prosperous nation that ensures New Zealanders everywhere have easy access to knowledge, that engages everyone in reading for pleasure, and that celebrates words as a valued part of our diverse heritage...".

In Thailand, however, RfP has not been officially discussed by the government as a potential approach to address the low-reading habit of Thai people (see section 3.2.2 The National Reading Policy). Previous Thai studies (TK Park, 2008; 2010) considered pleasure solely as the outcome of reading, not also as a means of increasing reading behaviour. Elsewhere, however, numerous studies have identified that RfP is one of the most effective ways libraries can enhance reading behaviour in both children and adults (Clark \& Rumbold, 2006; Duncan, 2013).

RfP has been implicitly mentioned in Thailand's reading surveys for a long time, as the National Statistical Office $(2010 ; 2012 ; 2014)$ defined reading as "the reading of all material out of studying and working hours including digital reading but excluding short messages (SMS) or e-mails." This suggests that when "reading" is mentioned in a reading promotion context in Thailand it includes RfP. Moreover, in the past 10 years, the active Happy Reading organisation, which focuses on promoting reading among underprivileged people and at community-level, has supported the RfP concept.

Paradoxically, the leisure reading concept and the word "pleasure" or "enjoyment" has not been mentioned at all in reading promotion guidelines by the Office of the Basic Education Commission (OBEC, 2011), the main authority responsible for school libraries in the country. The OBEC (2011) presentation at the 2011 Thailand Conference on Reading confirmed the arguments from two scholars, Eoseewong (2011) and Ngamwittayaphong, (2011), that school or formal education is the major cause of the non-reading habit of Thai children. The formality and repeated focus on "knowledge" and "life-long learning" in school reading promotion have unintentionally yielded the reverse outcome. A qualitative study by TK Park (2008) partly confirmed this, identifying that negative experiences at a young age, caused by excessive reading demands from parents and teachers, result in non-readers. 
Therefore, RfP is a crucial concept to explore as a potential basis for more successful reading promotion in Thai society. However, it is academically underexplored in Thailand, especially in non-formal educational settings which have the potential to engage more people in RfP, according to Krashen's low anxiety reading concept. This study focuses on four types of CLs in different areas of Northeast of Thailand where RfP is being applied.

\subsubsection{Research Questions}

The research questions are:

1. What are the factors affecting reading for pleasure practice in community libraries in the Northeast region of Thailand?

2. How do these factors facilitate or hinder reading for pleasure practice in community libraries in the Northeast region of Thailand?

\subsubsection{Research Objectives}

The objectives of the study are to:

1. identify the factors affecting reading for pleasure practice in community libraries in the Northeast region of Thailand

2. determine how these factors affect the reading for pleasure practice in community libraries in the Northeast region of Thailand

\subsection{Research Contribution}

\subsubsection{Contribution to Theory}

The study makes a significant contribution to RfP theory and community librarianship in the specific context of Thailand. It should be noted that despite the fact that the RfP is an explicit approach underpinning the development of the Thai public library as demonstrated by the establishment of the ultra-modern TK Park, a 'living library' in a luxury mall in Bangkok (Kongrut, 2012), the term has been rarely used by Thai scholars. Therefore, this study facilitates the understanding and clarification of the term and concept within the Library and Information Science (LIS) community context in Thailand. 
In terms of community librarianship, the research adds to the body of knowledge on CLS in developing countries. Although CLs have emerged as a new type of library, there has been only one study on a specific type of $\mathrm{CL}$ in Thailand, that under local government (Changket, 2011). The study, therefore, contributes to CLs literature by investigating the RfP practice of other types of CLs in Thailand defined by their establishing bodies: 1) an expert organisation (Wicha Association - WA), 2) ONIE, 3) health promoters, and 4) individuals.

\subsubsection{Contribution to LIS Research and Methods}

As Hoq $(2014$, p.14) suggested in his extensive review of literature on rural libraries and information services in Asia and Africa, most studies tend to discuss the issue in "a rather simplistic and linear view." Therefore, this study applies a qualitative approach to look critically into reading promotion practice in context. To extend knowledge of reading promotion in context, the study applies sociocultural perspectives, which are not commonly found in reading promotion research (Asselin \& Doiron, 2013).

In literacy research, the sociocultural perspective is also not as common as psychologicalcognitive approaches, which treats literacy as an isolated intellectual ability without context. There is a large body of educational research on reading in the psychologicalcognitive tradition in Thailand, as synthesised in Phosri's thesis (2014) as well as studies on Thai reading behaviour by TK Park (2008; 2010) (see 2.5.4.1 and 3.2.1.1). Therefore, the study takes the sociocultural approach, which not only generates more in-depth and context-based findings but also contributes a new perspective to LIS qualitative research methods (in Thailand).

\subsubsection{Contribution to Practice}

As a professional field, the contribution to practice tends to be the ultimate goal of most LIS research (Wildemuth, 2009). As there have been no studies about the four types of quite independent CLs or RfP practice in reading promotion in Thailand previously, this study contributes to the understanding of community-based reading promotion practice in Thailand. The study explores not only the perception but also the contextual 
circumstances of both professionals and non-professionals who act as library practitioners in this developing country.

The study supports the Thai government's attempts to identify appropriate practices to promote reading behaviour amongst the Thai population in the most disadvantaged and largest part of the country, the Northeast. It explores the possibility that a deeper understanding of factors contributing to the success of RfP could help narrow the educational and socioeconomic disparity between Isan and other regions.

\subsection{Structure of the Thesis}

The thesis has 11 chapters. After this introductory chapter, chapter 2 provides an overview of relevant literature elaborating on the two major concepts in the research, RfP, and CLs. Chapter 3 reviews the literature on the specific Thai and Isan contextual factors affecting RfP in CLs. Chapter 4 outlines the methodological approach utilised in the research following by chapter 5 outlining the physical characteristics, ethnic, and economic setting of the Isan region. Details of sites, participants, and the context of the eight case study sites are also presented. Chapter 6-9 reports case findings then the cases are crossed-analysed and presented in chapter 10. Chapter 11 synthesises the major findings of the study and, examines each factor through the conceptual lenses used and literature described in chapters 2 and 3 . Research contributions and future research are also presented.

\subsection{List of Publications}

1. Boonaree, C., Goulding, A., \& Calvert, P. (2018). Community library spaces for promoting reading for pleasure in Thailand. Paper presented at IFLA WLIC 2018, 24-30 August, 2018. Kuala Lumpur, Malaysia.

2. Boonaree, C., Goulding, A., \& Calvert, P. (2018). Opening minds: The Thai literacy traditions affecting reading for pleasure. Paper presented at The 1st International Conference on Library and Information Science, 17 - 18 August, 2018. Bangkok, Thailand.

3. Boonaree, C., Goulding, A., \& Calvert, P. (2017). Community library strategies for promoting reading for pleasure in Thailand. Paper presented at The 8th Asia-Pacific 
Conference on Library and Information Education and Practice (A-LIEP) 13-15 November, 2017. Bangkok, Thailand.

4. Boonaree, C., Goulding, A., \& Calvert, P. (2017). Reading for pleasure (RfP) and literacy problems in Thailand. Thai Library Association Research Journal. 10(2), 36-48.

5. Boonaree, C., Goulding, A., \& Dorner, D. (2015). Reading for pleasure practices in community libraries in Thailand. Paper presented at The 16th General Conference Congress of Southeast Asian Librarians (CONSAL XVI). 11 - 13 June, 2015. Bangkok, Thailand. 


\section{CHAPTER 2 Key Concepts in the Literature}

\subsection{Introduction}

This chapter provides an overview of relevant literature elaborating on the two research themes identified in Chapter 1; reading for pleasure (RfP), and community libraries (CLs). This chapter firstly synthesises studies on RfP. The major concepts used as lenses in the study are: 2.3) Krashen's Free-Voluntary Reading concept; and 2.4) Asselin \& Doiron's Ecological Framework for Community Library Development. The latter half of the chapter describes important elements in CLs affecting RfP (2.5) and key individuals involved in RfP (2.6).

\subsection{Reading for Pleasure}

Reading for Pleasure (RfP), an academic term for leisure reading (Duncan, 2013), has been identified as a tool to overcome low socioeconomic status (SES) (Krashen, 2011; OECD, 2010; Sullivan \& Brown, 2013). CLs can support one of the principles of RfP theory, Free Voluntary Reading (FVR). This section defines RfP, explores the relationship of RfP with reading attainment and reading behaviour, and discusses FVR.

\subsubsection{Defining Reading for Pleasure}

Clark and Rumbold (2006, p.5) aggregated extensive research evidence on RfP focussing on the United Kingdom where the authors say that inequality persists and literacy development is relevant for poverty eradication. They defined it as "reading that we do with our free will anticipating the satisfaction that one will get from the act of reading... It typically involves materials that reflect our own choice, at a time and place that suits us". Duncan (2013, p. 5) added that RfP focuses on the purpose of reading for leisure and "usually denotes an individual, silent activity ... and often indicates that reading will not be followed by activities or assignments common to reading in many educational settings". Therefore, if students are given reading to do followed by some sort of assessment task, by their definition it is not RfP. 


\subsubsection{Reading for Pleasure and Reading for Instruction}

As reading is generally treated as an academic skill, in Thailand's school teachers tend to teach students to read for instruction (RfI). Thus, RfP is a term used to differentiate between these two orientations. RfP is different from Rfl because RfP aims to nurture the personal will to read when the person engages in reading voluntarily. Regardless of the fact that they are different, the two types of reading have a significant interrelationship. RfP is the aim of $\mathrm{Rfl}$, and helps sustain reading behaviour in children (Clark \& De Zoysa, 2011). In addition, developing a habit of RfP is an indicator of reading skill (Williams \& Wavell, 2001). The United Kingdom Literacy Association's (2008) explanation of the differences between RfP and RfI is presented in Table 2.1.

Table 2.1 Reading for pleasure and reading for instruction

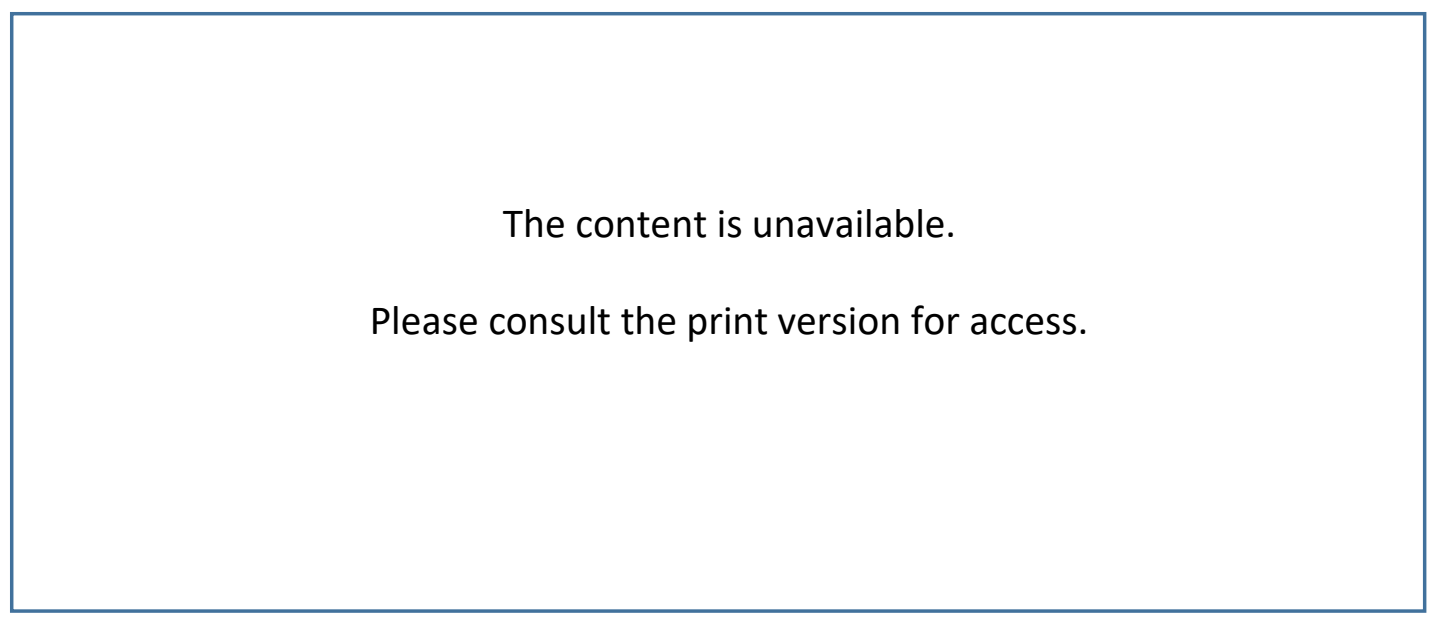

(Source: The United Kingdom Literacy Association, 2008)

A quantitative study of the relationships between reading enjoyment, reading attitudes, behaviour and attainment, indicated that reading enjoyment is twice as effective as reading attitudes in positively influencing reading behaviour (Clark \& De Zoysa, 2011). The desired reading attainment (Rfl) originates primarily from enjoyment through reading behaviour. The model is reproduced in Figure 2.1 (with bolder paths indicating stronger relationships). This finding was supported by an in-depth study confirming that reading enjoyment strongly affected reading attainment (Clark \& Douglas, 2011). 
Figure 2.1 Model of relationships of reading enjoyment, attitudes, behaviour and attainment

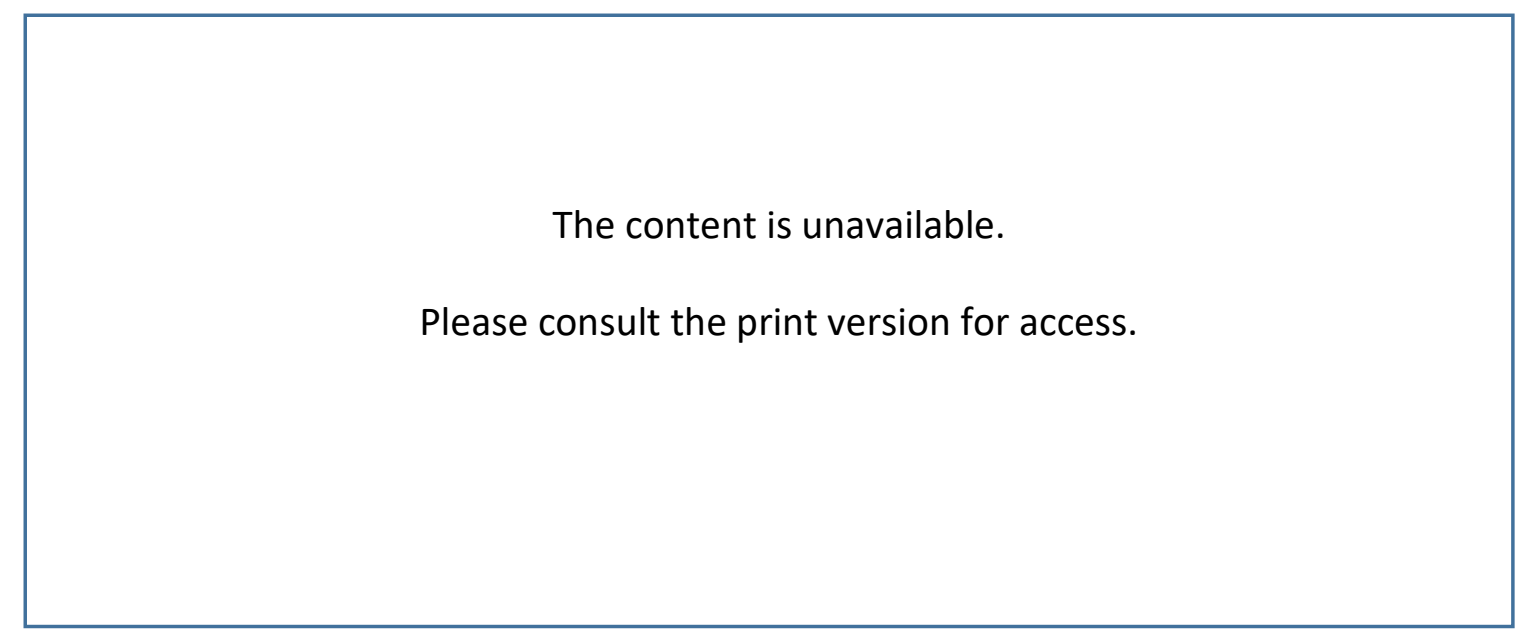

(Source: Clark \& De Zoysa, 2011, p. 5)

\subsection{Free-Voluntary Reading (FVR)}

Stephen Krashen coined the term "Free Voluntary Reading" or FVR in 1993. He defined FVR as "reading because you want to, self-selected reading for pleasure" (Krashen, 2014a). The FVR concept provides a useful lens to investigate the problem of this study because it focuses on reading enjoyment, the missing component in reading behaviour within the Thai population according to TK Park (2008; 2010). Moreover, Krashen is a linguist who advocates for book access and library access for children of low SES, which is at the core of this study (Krashen, 2016; 2014a; 2014b). In addition, his language education theory of second language acquisition, the Monitor Model, focusing on comprehensible input, and positive low anxiety feelings (the affective filter) is well connected to this study (see 2.3.2).

In this section, two constructs of FVR: 2.3.1) Key Concepts; and 2.3.2) FVR, Book, Access and Light Reading Materials are presented. Subsequently, the relevant language acquisition principles are synthesised in 2.3.3 Monitor Model, 2.3.4 Early Literacy Learning, and 2.3.4 Phonics Instruction. 


\subsubsection{Key Concepts}

Based on the Comprehension Hypothesis that says humans learn language by understanding it, FVR challenges the traditional Skill-Building approach (phonics, grammar, vocabulary, and spelling). A key principle in FVR is the accessibility of understandable text in a low-anxiety situation and the omission of accountability (Krashen, 2011a).

Krashen (2011a) proposed four hypotheses about FVR that focus on: 1) forgetting; 2) effortless reading; 3) unawareness of acquisition; and 4) the omission of comprehension checking. These hypotheses suggest reading materials need to be easy and interesting so that readers feel no obstacle to reading and concentrate only on the message. The readers will feel flow or become 'lost in the book' so that they forget that the materials contain new grammar and vocabulary. As a result, readers will be unaware of their language acquisition. The last hypothesis is crucial, as comprehension checking will force readers to memorise the text with the outcome that they do not 'forget', and therefore do not flow with the text. Thus, they will be less engaged in reading and will not enjoy it. Krashen believed that FRV is a route to language acquisition, either in a first or second language. In schools in the U.S.A. FVR was known as Sustained Silent Reading (SSR). Krashen (2011a) provided guidelines for SSR: 1) information resources; 2) activities; and 3) accountability and rewards.

\subsubsection{FVR, Book, Access and Light Reading Materials}

Over 20 years of advocating for FVR, Krashen (2011b; 2014; Krashen, Lee, \& McQuillan, 2012) has emphasised the need for libraries for children from low SES backgrounds. He believes that FVR and library access are the most powerful means to help children in poverty develop their literacy skills. Pribesh, Gavigan, and Dickinson (2011) examined school library media centres in North Carolina and Virginia and found evidence for Krashen's assertion that children from low SES have less access to libraries than those from better off families. The library access gap was found in the number of library personnel, annual new acquisitions, and library hours. 
The three aspects of library access and resources identified by Krashen (2011a) as important for encouraging FVR are as follows:

- Provide access to a great variety of books and other materials.

- Put minimum censorship on materials.

- Acquire comic books, magazines, easy books, hard books, and books written for language students of different levels.

On censorship, Krashen and Ujiie (2005) found the assumption that only quality literature should be selected for reading runs contrary to the reality that students do not generally like to read those books, so by providing only this type of reading material libraries and schools may miss the opportunity to turn students into avid readers.

Krashen strongly supports light reading as he believes that bestsellers, series novels, magazines, or comic books engage readers to read more and flow with the text, so they naturally gain literacy development naturally. His belief in the value of light reading materials is reflected in his extensive works, for example Mason and Krashen (2017), Cho and Krashen (2016), and Choi and Krashen (2005).

Krashen (2004) also emphasised that access to light reading in libraries is important for lexicon development in children. He suggested that "light reading can serve as a conduit to heavier reading" (p. 103). A comparison of common and uncommon words/found in children's conversation and printed materials revealed that a variety of light reading material encouraged the development of vocabularies, and children's books provide nearly three times (30.9\%) rarer words than adult conversation with children $(9.9 \%)$ (Hayes \& Ahrens, 1998).

\subsubsection{The Monitor Model}

The affective aspect of FVR links to an aspect of Krashen's second language acquisition theory, which can possibly explain why Isan people find it hard to learn Central Thai (CT) language. Therefore, this research also uses this aspect of second language acquisition theory which is focused on illiteracy problems in disadvantaged Isan rather than considering it as a reading behaviour problem. 
The affective aspect of a low anxiety environment is not described much in FVR but explained more extensively in Krashen's theories of second language learning, the Monitor Model. In this Krashen says that different learners perceive or interact differently to comprehensible linguistic input as the input is filtered out by each individual's affective filters, for example, their anxiety, poor self-esteem or low motivation. Learners with a low affective filter will feel more comfortable and will be efficient language learners, whereas learners who have a high affective filter will feel uncomfortable interacting with others and embarrassed when making mistakes, for example, and therefore will not learn the language as efficiently.

FVR and the Monitor Model are, in fact, difficult to separate from each other. Krashen insisted that: "When second language acquirers read for pleasure, they can continue to improve in their second language without classes, without teachers, without study..." (Krashen, 2004, p. 147).

\subsubsection{Early Literacy Learning}

Krashen believes that in terms of human literacy development, there is no difference between our first language acquisition and other subsequent languages. The fundamental way we acquire language ability is by listening attentively to spoken language that is meaningful to us. His FVR encourages adults to read or tell stories to children, stating:

\footnotetext{
Parents need to know that children will get far more benefit from being read to, from seeing parents read for pleasure, and from reading comics, graphic novels, magazines and books than they will from working through workbooks on sale at the local drug store (Krashen, 2004, p. 151).
}

Recently he emphasised the importance of storytime by putting storytelling and readaloud as the first of three stages to develop 'high levels' of literacy (Krashen, 2018, 16). He contended that storytime is a necessary preparation for FVR and later narrow reading that suits the individual's interest. 


\subsubsection{Phonics Instruction}

This research primarily employs Krashen's FVR lense that aims to enhance "reading behaviour" in the general population who can read by themselves, not for beginners who, he identified in his guidelines, "need other kinds of comprehensible text" (Krashen, 2014 , p. 10). Therefore, not every aspect of Krashen's FVR principles applies to young children aged 0-5, who generally cannot read, nor children who come from low SES families and tend not to be read to. These children may need explicit phonic reading instruction when they enter school.

The relative benefits of whole language (discovery-based) and phonic (structured) teaching approaches have been an on-going debate in Australia, New Zealand, the UK and the U.S. (Chapman and Tunmer, 2015; Tunmer, Chapman, Greaney, Prochnow \& Arrow, 2013). Hempenstall (2016) reviewed extensive scientific evidence on the teaching of reading and found that systematic explicit, structured reading instruction is generally more effective than discovery-based approaches (constructivism), and specifically important for "children at risk of reading failure". Schwartz (2015), however, recommends a balanced approach, suggesting that the integration of both whole language and traditional phonics-based interventions is usually most effective for early literacy development.

This study also uses the concept of emergent literacy which views literacy as a developmental continuum, starting in social contexts before formal literacy instruction. In summary, this study primarily employed FVR, a whole language approach, as a conceptual lens to explore RfP practices but was also mindful that phonics instruction can be an important intervention for children of low SES in disadvantaged Isan.

\subsection{The Ecological Framework for Community Library Development}

The second concept, Asselin and Doiron's Ecological Framework (2013), provides lenses for both $\mathrm{CL}$ operation and RfP in rural African areas, which has some similarities with the Isan context. Elements in the framework were found to be crucial factors affecting the sustainability of CLs in Malaysia (Abu, 2014), and Indonesia (Håklev, 2010). 
The Ecological Framework is a guideline for CLs to position themselves as "central players in personal, social, cultural and economic improvement in their communities" (Asselin, Abebe \& Doiron, 2014, p. 5). The framework identified four components: 1) context/ environment; 2) inclusion/equity/social justice; 3) partnerships /interactions; and 4) action/research. Although their model is abstract compared with CL models proposed by previous authors (i.e. Mostart, 1998; Dent, 2006; Ahmed, 2010), the model identified a sociocultural perspective to explore literacy development. The focus on community participation is the same principle that READ Global, the Library of Congress Literacy Awards 2014 winner for READ Nepal (READ Global, 2015), used when establishing their enterprise-model CLs in South Asia (Shrestha \& Krolak, 2015).

Later, Asselin, Abebe and Doiron (2014) demonstrated the Framework's clearer application in a training initiative for staff of CODE-Ethiopia CLs. Previously, the training programme had focused on library management and collection development. However, in its 2012-2016 phases, the initiative added a unit on a reading promotion programme (RPP) and literacy support principles and strategies using the Ecological Framework. The training also resulted in the seven standards of library programme development (2014, p.9) The standards and the application of the framework identified the key elements in the Ecological Framework which in fact match with important community library management elements identified by previous studies as mapped in the last column of Table 2.2.

Asselin and Doiron (2013) noted that CLs are unique in nature and therefore preferred to use the word "effective" rather than "best practice" in their suggested solution for reading promotion in CLs. They proposed five effective practices in reading promotion and literacy development which focus on RfP before proposing their Ecological Framework for ongoing $\mathrm{CL}$ developments. Their effective practices are: 1) choice of reading material; 2) opportunity to read voluntarily and independently; 3) supportive physical and social atmosphere; 4) reading models; and 5) opportunity to share reading. Their practices align with FVR principles, and also with Ngamwittayaphong's (2011) study of the promotion of a reading culture in the Thai context, discussed in 2.6.4. and throughout Chapter 3. The practices are mapped with italics in Table 2.2. 
Table 2.2 Mapping of $\mathrm{CL}$ management elements, Ecological Framework elements, and effective practices in reading promotion and literacy development elements

\begin{tabular}{|c|c|c|}
\hline $\begin{array}{l}\text { Community } \\
\text { Library } \\
\text { Management } \\
\text { Elements }\end{array}$ & $\begin{array}{c}\text { Elements in Ecological Framework } \\
\text { (1=context/environment, } 2=\text { inclusion/equity/ } \\
\text { social justice, } 3=\text { partnerships/interactions, } \\
4=\text { action/research) }\end{array}$ & $\begin{array}{l}7 \text { Standards of CL Programme } \\
\text { Development } \\
\text { (for librarians) }\end{array}$ \\
\hline \multirow{2}{*}{$\begin{array}{l}\text { Library } \\
\text { materials }\end{array}$} & collection to suit the needs of all people (2) & (1. choice of reading material) \\
\hline & $\begin{array}{l}\text { interaction with community members to identify } \\
\text { issues to be solved, and responding to community } \\
\text { needs with materials and programmes (4) }\end{array}$ & \\
\hline \multirow{4}{*}{$\begin{array}{l}\text { Responsive- } \\
\text { ness to local } \\
\text { needs }\end{array}$} & $\begin{array}{l}\text { libraries' role in inclusion and responsiveness to } \\
\text { changing social and political values (2) }\end{array}$ & $\begin{array}{l}\text { ability to include activities for } \\
\text { girls/women and families }\end{array}$ \\
\hline & programmes that include all people (2) & \\
\hline & $\begin{array}{l}\text { attempts to meet the needs and interests of } \\
\text { community members ( } 3 \text { ) }\end{array}$ & \\
\hline & library staff able to undertake users studies (3) & \\
\hline \multirow{3}{*}{$\begin{array}{l}\text { Library } \\
\text { Activities } \\
\text { /reading } \\
\text { promotion } \\
\text { programme } \\
\text { (RPP) }\end{array}$} & $\begin{array}{l}\text { librarians share results of activities with the } \\
\text { community and identity new ways to build their RPPs } \\
\text { (4) }\end{array}$ & $\begin{array}{l}\text { (2. opportunity to read } \\
\text { voluntorily and independently) } \\
\text { (5. opportunity to share } \\
\text { reading) (4. reading models) }\end{array}$ \\
\hline & $\begin{array}{l}\text { developing action plans, seeking feedback on the } \\
\text { actions taken and revising the action plan in a } \\
\text { continuous cycle of reflection, planning, action and } \\
\text { back to reflection (4) }\end{array}$ & $\begin{array}{l}\text { - ability to complete a variety } \\
\text { of literacy activities in the } C L \\
\text { - ability to create a detailed } \\
\text { plan for a RPP }\end{array}$ \\
\hline & librarians must document their activities (4) & \\
\hline \multirow[t]{3}{*}{ Staffing } & Library Management Committee roles (1) & \\
\hline & $\begin{array}{l}\text { library staff change their book guardians image to } \\
\text { that of learners, leaders, promoters and researchers } \\
\text { (3) }\end{array}$ & $\begin{array}{l}\text { ability to use CODE Ethiopia } \\
\text { Books effectively in reading } \\
\text { promotion programmes }\end{array}$ \\
\hline & $\begin{array}{l}\mathrm{CL} \text { operates in welcoming ways and is inclusive for } \\
\text { diverse gender, cultural, and linguistic needs ( } 2 \text { ) }\end{array}$ & $\begin{array}{l}\text { (3. supportive social } \\
\text { atmosphere) }\end{array}$ \\
\hline Training & training workshops for librarians (2) & $\begin{array}{l}\text { an understanding of librarian } \\
\text { roles in reading and literacy } \\
\text { supports for schools }\end{array}$ \\
\hline \multirow[t]{2}{*}{ Site selection } & physical location as a centre of community (1) & \multirow{2}{*}{$\begin{array}{l}\text { (3. supportive physical } \\
\text { atmosphere) }\end{array}$} \\
\hline & $\begin{array}{l}\text { CL provides safety, and is accessible to schools and } \\
\text { community services (1) }\end{array}$ & \\
\hline \multirow{3}{*}{$\begin{array}{l}\text { Community } \\
\text { participation/ } \\
\text { Coordination }\end{array}$} & $\begin{array}{l}\text { strategic positioning of library as a key component of } \\
\text { community development (1) }\end{array}$ & \multirow{2}{*}{$\begin{array}{l}\text { an understanding of the role of } \\
\text { the library and the librarian in } \\
\text { the community }\end{array}$} \\
\hline & $\begin{array}{l}\text { library staff forge meaningful partnerships and work } \\
\text { with their community stakeholders, for example } \\
\text { primary and secondary schools teachers ( } 3 \text { ) }\end{array}$ & \\
\hline & $\begin{array}{l}\text { library staff are capable of building community } \\
\text { connections and demonstrating the vital role of the } \\
\text { library in education and community development (3) }\end{array}$ & $\begin{array}{l}\text { ability to monitor and share } \\
\text { successes from RPP }\end{array}$ \\
\hline Budget & Library Management Committee roles (1) & \\
\hline
\end{tabular}




\subsection{Community Libraries}

This section reviews the literature on community libraries by dividing it into six parts:

1) defining community libraries; 2) community libraries in the developing world; 3) community libraries in Thailand; 4) reading materials; 5) reading activities; and 6) community participation. In each subsection, general concepts are discussed before focusing specifically on studies in the Thai context.

\subsubsection{Defining Community Libraries}

Stranger (2014, p. 93) defined community libraries (CLs) broadly as "a library, or a librarylike collection and lending service, that is not part of a public library program and is run by one or more people from the community it serves". This definition is broader than Shrestha and Krolak's (2015, p. 403) explanation of characteristics of these libraries, i.e., that they are small in size, not funded by the government, target newly literate people, and are mostly located in rural areas.

These two definitions are based on the African context where CLs generally have three key characteristics: 1) the library was fully or partly community initiated; 2) the library materials and services are provided to satisfy the information and literacy needs of its community; and 3) the library seeks to cooperate with the community and schools and establish a sense of ownership among community members (Stranger, 2014). Therefore, reading materials, reading activities, and community participation are key areas of CLs that are discussed further in 2.5.4, 2.5.5, and 2.5.6.

In the Thai context where most CLs have not been initiated by the community. Changket (2011, p. 36) defined and categorised CLs in her study by the nature of their operating agencies and selected only libraries under local government administration in six areas of Thailand. Thus, the CLs in her study included district libraries that were part of public library provision.

This study, however, uses Stranger's (2014) broad definition for several reasons. First, although the context of CLs in Africa is different from that in Thailand, the definition broadly covers most types of CLs in Thailand. Although some CLs in the study (CL3 and CL4) were established by ONIE, they were not fully funded by the public library system 
which is different from those in the West. Second, this definition clearly distinguishes 'community library' from 'rural library services', the closest heading listed in the International Encyclopaedia of Information and Library Science (2003). 'Rural library services' is defined there as a service that "operates in an environment of geographical remoteness, characterized by small population units and consequent modest financial support" (p. 558).

The two terms are closely related as they share one crucial similarity: the target area. Therefore, some authors may use them interchangeably, or use additional terms to be more specific, for example, rural community library, rural information services, or rural information provision. Commonly, CLs are located in rural areas; however, CLs also exist in deprived urban areas and have played a significant role in improving access to reading for the urban poor population (Pyati \& Kamal, 2012). Thus, this study includes two CLs (CL2 and CL8) in deprived urban areas.

\subsubsection{Community Libraries in the Developing World}

Since the 1970s, social movements in marginalised regions of the world, where public library systems have generally not been effective, have mobilised communities to establish their own libraries (Shrestha \& Krolak, 2013). In South Africa, the role of CLs in tackling inequalities has been manifest. Historically, CLs in Africa have been established as an alternative to post-colonial public libraries which, it is argued, cannot serve the information needs of contemporary African populations (Alemna, 1995). Chisita and Chiparausha (2017) found that rural African people have the perception that public libraries are expensive and only for the literate elite, hindering them from accessing the libraries. In addition, some library staff were ineffective in their communication with rural people because they tend to favour the elite and lack cultural orientation. In Nicaragua, public libraries also maintain an academic orientation, strict rules and do not provide materials to read for pleasure (Ndumu \& Mon, 2018).

These are the reasons that CLs can better serve community needs. Asselin and Doiron (2013) concluded that CLs represent the newest type of libraries in the developing world. An IFLA publication, Global Library and Information Science (2017) confirmed their 
conclusion by mentioning that CLs are one of the "New Initiatives for the Provision of Public Libraries" in Africa (Chisita, \& Chiparausha, 2017, p.35).

In Asia, Håklev (2010a; 2010b) explored the CL movement in Indonesia and found that Chinese book rental shops, pop culture in certain areas, and individual commitment contributed to the flourishing of the 'reading gardens' movement in the country. In India, Pyati and Kamal (2012) found that the library was an important positive space for the urban poor community in Bangalore.

\subsubsection{Community Libraries in Thailand}

Cheunwattana's (1998) study identified that the public library system was not responsive to the diverse needs of rural people and called for the reorganisation of the system. Alternatively, she argued, rural development policy needed to be added to the existing public library goals. She mentioned both the functional and recreational purposes of public libraries and emphasised that there was a need for appropriate reading material, cooperation and greater community involvement and empowerment.

As mentioned in 1.2.2, during the period 1972-1999, ONIE established village reading centres and started to operate mobile libraries in several forms. In the Isan area, the Bookmobile Programme is operated by the Department of Library and Information Science, Khon Kaen University; it is one of the oldest outreach library services for rural school children in Thailand (Cheunwattana, 2003).

In 2001, the ONIE's reading centres with newspaper subscriptions were administratively transferred to local Tambon Administration Organisations (TAOs). In 2003, however, to maintain its role in adult education, ONIE initiated a new form of CL called "Smart Book Houses" (SBHs). These have been established in 41,800 villages in the country some revived from the old village reading centres. ONIE sought to find premises provided by local volunteers and supplied them with daily newspapers, a weekly review magazine, and a monthly magazine. Therefore, they were aimed at improving adult literacy.

However, in 2016, the ONIE subscription budget for SBH project was cut by the Office of the Auditor General of Thailand (OAGT). The OAGT investigated the daily newspaper subscriptions and distribution and found that the use of money was ineffective because 
large number of $\mathrm{CL}$ subscriptions duplicated those that the TAOs had been subsidising since 2001. By law, TAOs were the local administrative authority that had been granted a fund to subscribe to newspapers for local communities, and not ONIE. After the budget cut, ONIE continued the project under the new name "Community Book Houses" and created the Book Voyage donation project instead.

\subsubsection{Reading Materials}

Reading materials are the most important technical element in RfP but many libraries in Thailand focus on bricks and mortar (Fry \& Bi, 2013). Sacchanand, Prommaphan and Sacchanand (2006) found that, in general, public libraries in the country have failed to meet minimum standards in several aspects, including the provision of reading materials. During the data collection period for this study (2015-2016), each public library was allocated only 20,000 baht ( 800 NZD) per year to acquire new titles.

\subsubsection{The Variety of Books}

A study of reading culture in Thailand by Ngamwittayaphong (2011) found that the number of books, the variety of books, reader freedom to select books, and a regular rotation of new books to supply readers, are enabling factors for a reading culture in the country. These are similar to the conditions underpinning FVR and the Ecological Framework. In addition, a study of book rotation systems in small primary schools in Thailand (Vongsirinavarn \& Pleehajinda, 2010) and book circulation in CLs (Kongrut, 2012) revealed positive results in the Thai context, suggesting variety in reading materials is a key factor encouraging RfP.

Krashen's FVR focus on the importance of a variety of reading material aligns with a study on Thai reading behaviour and the reading index (TK Park, 2010) that revealed in all 16 reading indices studied through three dimensions: 1) reading behaviour; 2) reading ability; and 3) reading outcomes, the most important indicator was reading comprehension, which is also the most important aspect in FRV theory. Moreover, the same study found that in the reading behaviour dimension, the most important factor for reading behaviour in Thailand, and the only significant indicator derived from second order confirmatory factor analysis, was the variety of books to read, and not any other indicators studied, for example, quantity of books, time spent on reading, and money 
spent on reading (TK Park, 2010). Therefore, it seems that to support RfP, CLs should have a great variety of books to suit the diverse reading abilities and interests of their target readers and it is beneficial if readers can find understandable and meaningful texts that suit their interests and ability to read without pressure.

A qualitative study of reading behaviour in an organic farming group in a district of an Isan province, revealed that where there was no village reading centre, participants were eager to borrow a variety of books, newspapers, and light reading materials, for example novels, comics and popular magazines, from neighbours, village schools, temples, and local hair salons (Wech-o-sotsakda, 2015). The study's findings suggest that reading policy-makers should realise that villagers' reading interests require a wide variety of reading materials. The study did not cover enough male participants to represent their reading interests, however.

In the rural Isan context, CLs established with the primary aim of supporting non-formal education also support students with their formal education. Boonaree and Tuamsuk (2013) found that the largest group of users of the learning resources organised by Buddhist monasteries in Isan were school students. Their use reflected the fact that the school libraries and public libraries in the area are inadequate and so students rely on any library available in their communities. In conclusion, CLs in Thailand have served almost all people in the community, including students in the formal school system.

\subsubsection{Book Donation}

Book donation has been documented as a problem in developing countries (Alemna, 1995; Sturges, 2014; Asselin, Abebe \& Doiron, 2014; Dent, 2006; Hoq, 2014). Studies in Thailand have also identified problems regarding the quantity and quality of both locally donated and acquired materials (Ahmed, 2010; Leowarin, 2010).

The former President of PUBAT criticised the poor quality of book donations to CLs and schools, which many Thai believed to be a form of Buddhist merit-making, and said it had not been an effective way to promote reading in rural villagers and a reading culture overall (Kongrut, 2017). Donated books in Thailand are often out-dated and not attractive to villagers. He asserted that in order to attract people to read, the mind-set of book donation needs to change radically. 


\subsubsection{Reading Activities}

RfP reading activities should occur in home environments at a young age because children who do not enjoy reading, tend not to read for pleasure when they grow up (Clark \& Rumbold, 2006).

\subsubsection{Control over Book Choice}

Krashen (2011a) identified that successful reading promotion for children should focus on their freedom to choose books, to stop reading whenever they want to and to read for short periods of time each day. These concepts confirm the United Kingdom Literacy Association's (2008) explanation of RfP because it reflects a child-directed orientation. This crucial aspect will produce a low-anxiety situation, a key concept in FVR.

The independence to choose books by themselves has been found to be crucial for teenagers' enjoyment of reading. Manuel and Carter (2015) found that for teenagers aged $12-16$ in Australia, $62.5 \%$ of female participants and $78 \%$ of male participants identified that the worst book choices came from teachers/class novel/compulsory books. Therefore, that balance between reader preferences and teacher selection is an important factor affecting RfP.

\subsubsection{Affective Aspect of Reading Activities}

Fletcher (2017) found that for young adolescents aged 11-13, the critical period for children to develop their interest in RfP, the right affective conditions are crucial and sociocultural environments similar to those in Ngamwittayaphong's study (2011) nurtured those conditions. It is good to provide creative support from teachers and adults to make young people feel that reading is fun and 'cool', and to provide access to a wide variety of books appropriate for their age. However, the most important factor for reading development is reading motivation, which Clark and Rumbold $(2006,14)$ defined as "the internal states that make people read". This allies with Krashen's FRV principle on motivating children to read because of their intrinsic interest and not by giving extrinsic rewards.

In addition, the sociocultural aspects of reading activities have been mentioned in the literature on CLs. Two of Asselin and Doiron's (2013) practices in the African context, the 
physical and social atmosphere, and the opportunity to share reading, are reflected in the desirable reading promotion activities in CLs identified by Ngamwittayaphong (2011). Activities need to be appropriate for the participants and sufficient to meet demand. Her case study also focussed on the affective aspects (delight, happiness, pride), local support from individuals and organisations, creative activities arranged to open more reading spaces, and appropriateness to age, gender, educational background, life context and community context.

\subsubsection{Accountability and Reward}

For accountability, Krashen (2013) suggested reading promoters should use minimum measures by not requiring book reports and tests. He explains that accountability will send messages of memorisation and distrust. He does not support the use of rewards for reading but advocates using direct encouragement because rewards send the message that reading is not pleasurable by itself and that we need to be bribed to do it (Krashen, 2013). The affective or 'voluntary' aspect, in his view, tends to increase not only reading comprehension but also positive attitudes toward reading. This notion holds true according to Clark and Rumbold (2006) who reviewed literature on RfP and found evidence that revealed that intrinsic motivation, i.e. reading activity through personal interest, "leads to conceptual understanding and higher level thinking skills, while extrinsic motivation tends to lead to 'surface' rather than deep learning" (p.16).

Therefore, if children read because of a reward, to get better grades, to please teachers or parents or seek recognition for success, it will not guarantee that they deeply understand the text, enjoy reading, and sustain their reading behaviour. In Thailand Ngamwittayaphong (2011) also identified that extrinsic rewards or marketing tactics introduced by private companies in reading promotion activities did not yield sustainable results.

\subsubsection{Community Participation}

Community participation and inclusiveness are important concepts in Asselin and Doiron's (2013) framework presented in section 2.4. Community participation also includes reading activity participation which not only helps individuals achieve personal pleasure and skills but also means that family and community can connect (Duncan, 
2013). Ngamwittayaphong (2011) stated that group gatherings bring enjoyment, support and sharing that will unintentionally nurture the love of reading similar to that in Malaysia (Pandian, 2011). The studies are discussed more in 3.2.2.2 Sharing Reading.

In some countries, for example Indonesia and Japan, civic movements such as Reading Gardens and Bunko have driven community reading promotion. Håklev (2010a) found that in Indonesia, the most successful type of reading gardens were the independent ones started by small NGOs (Non-governmental organizations) or citizens' groups, not those set up by the government or run by local or regional government. In Malaysia, Abu (2014) identified that the management of rural public libraries in Malaysia takes a topdown approach that is not responsive to the needs of rural communities. In Thailand, Ahmed (2010) found that the community-led library services have had a beneficial impact for rural people. These studies established significant empirical findings that the keys to success in sustaining CLs are community participation, connectedness/ inclusiveness, civic involvement, and the capacity building of local information workers, and not solely money.

\subsection{Key Individuals in RfP}

Public librarians generally play a major role in RfP. However, most CLs in this study are informal organisations involving a range of individuals who facilitate book access within the community. The key individuals in RfP in CLs in Thailand discussed in this section are: 1) Leaders in RfP; 2) Family Members; 3) Teachers; and 4) Reading Promoters.

\subsubsection{Leaders in RfP}

In Asia, Håklev (2010a; 2010b) found that individuals' commitment helped the CL movement in Indonesia flourish. Similarly, in Thailand, there are individuals who generally have higher education than local reading promotors and seek resources, specifically quality reading materials for the CLs, and also provide educational support impacting on villagers' attitudes toward RfP. Renowned authors, for example Cheewan Wisasa, and a National Artist in Literature, Makut Ornrudee, are examples of leaders in RfP. They are committed not only to produce high-quality children's books but also to initiate creative projects supporting literacy development throughout Thailand. 
Makut Ornrudee initiated a CL project in Muslim mosques in rural Southern Thailand. In the successful project, eight mosques were provided with 7,000 new books and he found a reading role model who used RfP to inspire community life. In addition, he has fought for and proposed many measures to promote reading among the general Thai population, although his proposal to the government for an independent body to regulate every aspect of the book industry and to encourage reading has not succeeded. He argued that when a majority of families are in poverty, reading behaviour should be the practical responsibility of school libraries and CLs, not the family (Kongrut, 2012). His beliefs about the roles of school libraries and the CL project combine well with Krashen's assertion about library and children in poverty - rural people read if there are quality books provided for them.

\subsubsection{Family Members}

Reading is a contextual and complex skill in which family and the home environment influence young children from birth through the rest of their lives (Fletcher, 2017). Evidence from around the world has shown that SES and parents' education greatly affects RfP (Dickenson, 2014). In low SES families, support from libraries and other programmes makes a difference because parents cannot always fully support their children's literacy development. Research evidence shows that poorer parents have higher levels of stress and anxiety, thus affecting their ability to support their children's early development (Cooper \& Stewart, 2013).

Home and the community provide opportunities to support emergent literacy skills, and thereby prepare young children for school, by reading to young children and having quality conversations with them (Anderson \& Fearnley-Sander, 2015). Family literacy, "the way parents, family and community members use literacy at home and in their communities" (Ohio Literacy Resource Center, 2015) is a significant contextual issue affecting RfP. The definition given does not consider "family literacy" as occurring only in the family home but also in the community because reading is a social activity (Adkins \& Brendler, 2015).

The supportive environments for early literacy development identified by Baumann and Duffy (1997), are: 1) print-rich environments; 2) language-rich environments; 3) 
knowledge-rich environments; 4) (RfP) nurturing environments; 5) home-school connection environments. Two important environments for RfP are as follows:

\footnotetext{
1) print-rich environments, where [children] are read to and given opportunities to read, where they see their caregivers read, have opportunities to engage in pretend play, are encouraged to interact with environmental print, and visit the public library; ...4) (RfP) nurturing environments, where they interact with a variety of people who believe that one purpose of literacy is to entertain (Baumann and Duffy, 1997, p. 22).
}

A study in Thailand also found that the development of an appropriate environment through which children can easily access books is crucial for increasing/nurturing reading behaviour (TK Park, 2010). This confirms findings from studies undertaken in other countries: that book access and a literate environment are the most crucial factors to grow reading behaviour (Clarke \& Douglas, 2011; Krashen, 2011; IFLA, 2007). Therefore, book provision, being role models, reading to young children and family literacy are four major factors influencing RfP.

Generally, people in poverty need to prioritise securing the necessities for sustaining life before education and leisure (Jongudomkarn \& Camfield, 2006). Thus, the provision of cheap access to books is key to nurturing reading activities in deprived areas. The reading culture in Japan which developed after WWII focused on providing equal access to quality reading materials for local communities (at village level) both in the form of subsidies to school libraries and CLs. Similarly, in Vietnam, the socialist government did not just develop library access in major urban areas but also spread book access to rural villages (TK Park, 2009).

\subsubsection{Teachers}

As noted above, Makut argued that, due to poverty, reading in Thailand should be promoted through practical programmes in school libraries and CLs (Kongrut, 2012). For people in poverty, teachers and librarians are crucial for mobilising RfP. Happy Reading. (2017c) also identified that in rural Thailand, teachers and education administrators are important stakeholders in creating a reading culture. However, not every teacher can play this role; to promote RfP successfully they need to have sufficient knowledge of reading and books for young people. 
Manuel and Carter (2015) identified that in Australia, students valued book selection by informed teachers. They suggested a balance of materials derived from four approaches to selection: teacher; teacher-student negotiations; student-student negotiations; and student self-selected reading material. However, in Asian societies, teachers normally play parental roles that require children to obey. Ismail (2014) identified this in Malaysia, and Dorner and Gorman (2011) identified it in Laos; teachers tend to have high Power Distance. Hofstede Insights (2018b) defines this as, "the extent to which the less powerful members of institutions and organisations within a country expect and accept that power is distributed unequally". Teachers treat their students more like their children, and students need to show high regard for teachers. With this hierarchical orientation, children are expected to obey and not argue with adults. Therefore, negotiations do not normally occur between teachers and students. This may have negative results such as silent resistance; for example, in TK Park's (2010) study, Thai youth asserted that they are non-readers due to the excessive demands for reading for instruction from parents and teachers during their childhood.

TK Park (2008) identified the encouragement of RfP in parents and teachers as one strategy to promote reading behaviour. The study recommended their development as strong role models who practice RfP, leading to the eradication of the attitude that reading is only for educational purposes. Most importantly, teachers and parents need to be aware that they cannot simply command children to read but they need to model good practice by reading themselves (Krashen, 2004; Asselin \& Doiron 2013).

In Thailand, Tamrackitkun (2010, p. 37) identified that the teacher's role as a reading model is an important element as the teacher is "an active member of the classroom reading community, demonstrating what it means to be a reader and the [intrinsic] rewards of being a reader". Intrinsic reward aligns with Krashen's assertion that reading is rewarding by itself and no other reward is needed. The teacher who is well informed and demonstrates positive reading behaviour will also be able to suggest further reading material to suit each student.

Krashen (2004) stated that “...when teachers are relaxing with a good book during SSR sessions, teachers are doing their jobs" (p. 151). Perhaps this is the most important and 
most difficult factor to change; a Thai Scholar, Lao (2013), commented on reading behaviour and the typical parental roles of Thai adults that:

First, I would like all adults to talk less and read more. Stop telling the kids that they read too little because a lot of the elite in our society, a lot of our policy-makers don't read enough either. Second, respect the kids. Children are sometimes smarter than adults.

Early childhood teachers and caregivers are particularly important persons as RfP needs to start in the early years. However, the quality of early childhood education, including the quality of teachers and caregivers in Thailand has been an issue. UNICEF (2016) identified that only $67 \%$ of some 20,000 early childhood development centres in the country passed the quality assessment survey conducted by the Department of Health in 2013.

\subsubsection{Reading Promotors}

Reading Promotors (RPs) receive training in RfP concepts from the leaders in RfP (section 2.7.1) and mobilise RfP activities in their communities. RPs in the study can be any persons involved with community reading promotion, for example, community library officers, village health volunteers, or youth volunteers. These individuals need to share some fundamental abilities, however. Ngamwittayaphong (2011) identified that RPs, as a mechanism to support and stimulate reading, needed to be pragmatic, have ability to make friends, have ability to analyse the context and situation to design appropriate activities, and have ability to promote the participation of target groups and related parties.

Ahmed (2010) found that the training of library committee members and staff members before libraries were established was crucial for the success of community-led library initiatives in Thailand.

Since the 1960s, the village health volunteers (VHVs) have been an important mechanism to link public health services and families in rural communities in Thailand. They have been a crucial factor in supporting early literacy development for young children, specifically children aged 0 - 3. Kauffman's (1997) ethnographic study of VHVs in Isan identified that due to urbanisation their general healthcare roles were becoming 
less important. However, they adjusted their practices to address "evolving social problems resulting from urbanization" (p 249). Kowitt, Emmerling, Fisher, and Tanasugarn (2015) found that VHV programmes in Thailand had significant strengths in terms of strong collaboration with public health officials, and community trust.

\subsection{Chapter Summary}

This chapter has reviewed relevant literature on concepts central to RfP that are used as lenses in this study to investigate CLs in the Isan area. Krashen's FVR concept is presented first as it the principal lens used to look at the phenomena in the study. Asselin and Doiron's Ecological Framework for Community Library Development is the secondary lens because it touches on most elements in FVR with additional sociocultural views and practices used in the CL context.

The latter half of the chapter presented CL concepts affecting RfP and the key individuals involved in RfP. These core elements are based on both international and Thai literature synthesised with FVR, the major lens in the study. Both supportive and contested aspects are reviewed to provide a realistic picture of the CLs phenomena and the application of the FVR concept in the Thai context. 


\section{CHAPTER 3}

\section{Contextual Factors and Their Literature}

\subsection{Introduction}

The contextual factors affecting RfP in Thailand are tightly interwoven with reading promotion policy and the sociocultural context underlying literacy problems. Research on drafting the National Learning and Reading Frameworks synthesised studies on reading behaviour in Thailand and revealed the complexity of the reading problem. At the same time, the learning tradition requiring Thai children to be obedient unintentionally discourages them from reading, thinking beyond expected norms, and asserting their different opinions (Hallinger, 2010; TK Park, 2018). Most importantly, some families take little interest in their child's development and reading activities. In the reading environment there are limited reading materials, in general libraries are few and of low quality, and teachers and librarians have limited knowledge about how to encourage reading. The lack of a national reading policy and preference for digital media has also been mentioned (Dechwittayapon, 2015). Therefore, it is nearly impossible to set clear boundaries between the contextual factors affecting RfP.

The first section in this chapter, 3.2 Thai Reading Context, synthesises studies on the Thai reading context involving: literacy problems; reading policy; and related activities at the national level affecting RfP. The next section, 3.3 Thai Cultural and Social Issues, identifies specific Thai sociocultural aspects, the impact of which may not have been fully realised previously because they are embedded in the way of life. The last major contextual factor, 3.4 Isan Socioeconomic Influences, covers specific factors influencing the way Isan people interact with literacy, reading activities, and library access.

\subsection{Thai Reading Context}

This section synthesises studies at the national level involving: 1) Literacy Problems in Thailand, 2) The National Reading Policy, 3) Cooperation between Supporting Organisations in Society, and 4) The Book Market. 


\subsubsection{Literacy Problems in Thailand}

Literacy problems are a complex social phenomenon in Thailand involving three major problems: 1) Reading Behaviour; 2) Illiteracy; and 3) Reading Attainment.

\subsubsection{Reading Behaviour Problem}

The reading behaviour problem is directly relevant to RfP. Clarke and De Zoysa (2011) defined reading behaviour as "the frequency, width and breadth of a person's reading". In their quantitative study, reading behaviour was measured by one variable, the frequency with which children read outside of class. Similarly, Ngamwittayaphong (2011) indicated two desirable behaviours: more time spent reading voluntarily and more diversity in reading, as the primary traits in reading culture in Thailand. Therefore, they agree that variation of reading materials is critical to nurture reading behaviour.

In addition, a conflict between the expressed values and actual behaviour of Thai people in relation to reading was revealed by a study of Thai Reading Index by TK Park (2010). The index derived from a mixed method study employed focus group, content analysis and second order confirmatory factor analysis. The reading outcome index was as high as 69.413 but the reading behaviour index was only 41.938 , which is lower than the reading ability index (63.654). The reading outcome statistics indicated that Thai people highly value reading outcomes, composed of five indicators arranged by the most to the least important: life skills; academic achievement; career achievement; adaptability (57.7-74.3\%); and pleasure (22.9\%). The percentages show that Thais think that reading will generally bring life skills, academic achievement, career achievement, and ability to adapt to changing circumstances. Only $22.9 \%$ of respondents value the pleasure or enjoyment that comes from reading. As they do not view reading as an activity that brings pleasure, Thai people tend to read less than people in countries who perceive it differently (TK Park, 2008; 2010). The 2010 study also confirmed the previous TK Park study (TK Park, 2008) that, in general, both young Thai people and their parents value reading as an advantageous activity in terms of: 1 ) knowledge; 2 ) spare time usage; 3 ) widening perspectives; 4) imagination and creativity generation.

The most recent study by the PUBAT (2015) revealed different results from previous research, showing that Thais are now reading books as a hobby and the first reason for 
reading is RfP and entertainment (49.2\%). The favourite genre (and contents) are comics/graphic novels (34.4\%), well-being (28.1\%), non-fiction (23.1\%), Thai novels (21.8\%), and travel (21.3\%). In addition, an important segment of the population, youth under 20 years of age, indicated that they mostly read for pleasure while people aged over 50 participated in RfP the least. This may signify that Thai society is in a transitional period regarding RfP.

\subsubsection{Functional Illiteracy Problems}

The Reading of the Population Survey by the National Statistical Office which has been undertaken five times since 2003, focusses on leisure reading in Thailand. The survey found that in 2014 Thais generally read slightly less than 2011. The notable figure was that $27.5 \%$ ( 3.1 million) of the population said that they do not read because they cannot (National Statistical Office, 2014). This figure was different from the national literacy rate, which was reported as high as 91\% in 2013 (The National Statistical Office, 2013), and the youth (age 15-24) literacy rate from the Multiple Indicator Cluster Survey (MICS) 2015-2016, which was $94.5 \%$ for females and $94.7 \%$ for males (National Statistical Office, 2016, p. 129). The number was high because MICS assessed literacy on a minimal test, that is, "the ability of the respondent to read a short, simple statement or based on school attendance." This roughly reflects the outcomes of basic primary education (ages 6 - 12) but does not show adequate reading skill and comprehension, important elements for RfP.

Based on the Programme for International Student Assessment (PISA) 2012 reading assessment data, nearly one-third (32\%) of 15-year-old Thai students (grade 9) scored below the minimum level of functional literacy. Moreover, $47.2 \%$ of these young Thai who were functionally illiterate were in village schools, while only $15.5 \%$ were in schools in large cities (Figure 3.1). 
Figure 3.1 Proportion of functionally illiterate 15 -year-olds in 2012 by type of location

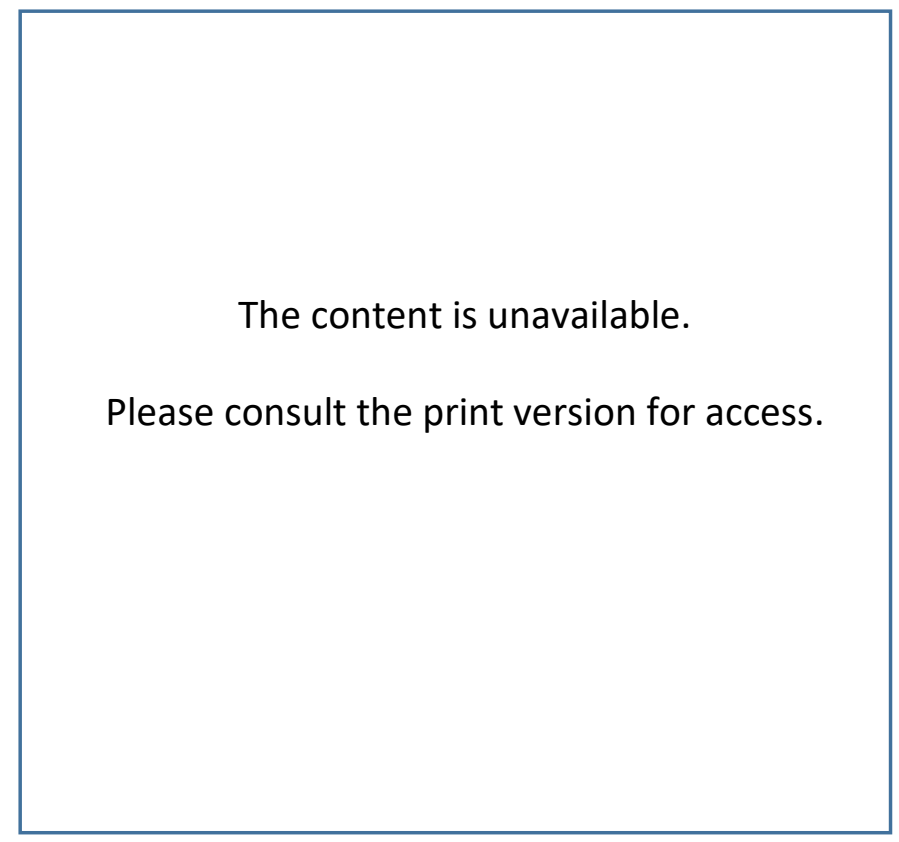

(Source: Lathapipat \& Sondergaard, 2015, p. 16)

\subsubsection{Reading Attainment Problem}

The low reading ability amongst Thai students was reported in PISA (Frederickson, 2016) as mentioned in Chapter 1. The analysis of PISA 2015 test scores by PISA Thailand (2015) confirmed that the continuing disparity between the quality of education provision in rural and urban areas in Thailand remains a major problem. An education system that is modelled on Bangkok schools is not practical nationwide. What is important for this study was that Thai students who study in rural village schools score extremely poorly for reading. Therefore, this is an area that RfP in CLs can help address, because Krashen (2011), OECD (2010) and Sullivan and Brown (2013) found that RfP is the most powerful mechanism to help children in poverty develop their literacy skills and escape low SES.3.2.2 The National Reading Policy3.2.2.1 National Learning and Reading Frameworks 2017Although reading promotions have been active in Thailand for 40 years, the country has never had a reading policy and the commitment of the government to reading promotion has been questioned (TK Park, 2008). A study of Thai reading promotion policy by TK Park (2009) identified that in the 20 years previously (1988-2008) reading 
policy in Thailand was based on the National Education Act 2003 and the 10th National Economic and Social Development Plan (2007-2011).

The Department of Cultural Promotion (2017) within the MOC wrote the National Learning and Reading Frameworks that were submitted to the Cabinet in late 2015. In August 2016, the name was changed to the Reading Culture Promotion for Thai Learning Society Framework 2017-2020 (B.E. 2560-2564). Four strategies (Table 3.1) were announced and related projects from authorities/organisations, for example the MOE, MOC, Ministry of Interior, local administrative organisations, and Happy Reading, were brought together to draft the action plan for the framework. Table 3.1 indicates the four strategies and their goals.

Table 3.1 Reading Culture Promotion for Thai Learning Society Framework 2017- 2020

The content is unavailable.

Please consult the print version for access.

(Source: Department of Cultural Promotion, 2017)

This framework is rather knowledge-oriented, focusing on knowledge and learning resources which align with the OBEC reading promotion policies presented in the next section. The 2017 frameworks make no mention of the leisure reading concept and the words "pleasure" or "enjoyment" in reading promotion. 


\subsubsection{Ministry of Education Policy}

As mentioned in 1.3 OBEC is responsible for school libraries. ONIE, another authority under MOE operate public libraries and CLs. However, the overall quality of education in Thailand and MOE's performance has faced severe problems for decades; educational reforms have been initiated and failed several times (Fry \& Bi, 2013).

Hallinger and Lee (2011, p.1) found that stagnation and instability in policy made educational reform rather "an impossible dream". There was reform stagnation, especially on a change in teaching approach from teacher-centred to learner-centred, and the strong cultural issue of top-down administration that fails to engage teachers in policy design and decisions. The instability in policy resulting from politicians' interference and frequent changes in leadership in MOE was identified as one of the most important factors affecting MOE policy (Hallinger, 2010). Recently, The Global Competitive Index 2017-2018 (World Economic Forum, 2018) reported that among 137 countries in the world, the quality of primary education in Thailand ranked $89^{\text {th }}$, and transparency of government policy making ranked $83^{\text {rd }}$.

Most importantly, in the past 10 years, MOE has not recognised that the RfP concept can be part of the solution to literacy problems in the country. A presentation on OBEC reading promotion policies at the Thailand Conference on Reading 2011, showed that reading policy in Thailand is derived from the 10th and 11th National Economic and Social Development Plans (NESDP) and focuses on learning (for academic and career purposes) and knowledge (OBEC, 2011). The presentation also identified that the Decade of Reading Agenda (2009 -2018) focused on: 1) Reading skills promotion; 2) Reading environment promotion; and 3) Social awareness promotion. As reading skills have been prioritised as the first focus, and there was no explicit inclusion of RfP, this tends to guide schools towards a reading attainment focus, which can obstruct RfP (The United Kingdom Literacy Association, 2008).

The Bookstart project initiated by the Books for Children Foundation (ThailBBY) with the support of the PUBAT, was mentioned in the OBEC presentation. However, it was a small scale project, not the nationwide book-gifting schemes to early-years children similar to Bookstart projects in the UK and Japan. 
Due to the severe problems of reading among students in grades 1-3 (age 6 - 8) the Ministry of Education declared 2015 to be "a year for no illiterate students" by setting a target of $100 \%$ of first grade students (ages 6 and 7) able to read and write. OBEC has reported progress on the project and the percentage of students who cannot read has clearly declined. It achieved this by using a variety of reading instruction approaches, for example phonics instruction (Ramasut \& Rohitsatien, 2016).

However, Happy Reading (2017a) reported that the percentage of children with literacy development problems was still high at $38.2 \%$, much higher than the $5-15 \%$ found in developed countries. This may be due to the focus of the two previously mentioned $10^{\text {th }}$ and $11^{\text {th }}$ NESDPs and the misguided ECD approach mentioned by the ECD drafting committee and discussed in 3.3.1.2.

In relation to $\mathrm{CL}$ initiatives, Cheunwattana (1999) identified that the implementation of reading promotion policy in rural areas depends on various factors, especially organisational structures and resources. In her case studies of rural library service under the government, several organisational frameworks focused on educational activities (literacy), thus putting leisure reading promotion activities as a lesser priority.

MOE, especially OBEC, receives a huge annual budget to support students in poverty. However, the problems affecting RfP seem to be not so much about having insufficient money to buy books, but inappropriate management arising from ineffective guidelines and supervision, a common problem in Thai government policy implementation (UNICEF Thailand, 2014). In 2016, eight years after the establishment of the "Decade of Reading Agenda", the president of PUBAT commented that the government had not put enough effort into promoting reading among children and young people (Suchiva, 2016).

\subsubsection{Happy Reading}

Allied with the Decade of Reading Agenda, The Reading Culture Promotion Programme (Happy Reading) was established in 2010 under the Thai Health Promotion Foundation, which is an autonomous state agency established under the Health Promotion Foundation Act 2001. Happy Reading is one of the Thai Health Promotion Foundation's master plans for health promotion and strengthening civil society, which are proactively and strategically executed through its partners nationwide. It actively supports the 
production of materials and reading projects both by NGOs and other agencies in rural areas around the country.

Figure 3.2 shows their Reading System for Healthy Living involving the public health and education systems. The Early Childhood Literacy Fund (ECLF) is the major focus promoted by Happy Reading. As there are no major organisations responsible for community reading in Thailand, the system shows that CLs need to work holistically with schools and health promotion organisations to promote RfP.

Figure 3.2 The Reading System for Healthy Living by Happy Reading

The content is unavailable.

Please consult the print version for access.

(Source: Happy Reading, 2015)

Happy Reading funded Ngamwittayaphong (2011) to undertake a qualitative study of factors affecting the promotion of a reading culture in Thailand. She defined the culture of reading as "the behaviour of continuous reading until it becomes the person's habit covering all of his/her lifestyle areas, in which readers see the value and benefits of reading hence also [to] teach or promote reading to others". In 2017, Happy Reading 
synthesised the experiences of 11 leaders in community reading, showing how their growth mind-set supports initiatives around the country (Happy Reading, 2017b). The publication contains the discussion of most of the factors reviewed in Chapters 2 and 3. In 2017 Happy Reading also played a vital role in ECD, for example, collaborating with the MOE and others to organise the event Building Thailand 4.0 by Reading to Young Children. It also introduced the "Smart Kids Smart Reading Home" project in 15 communities around the country. This project aims to: 1 ) identify reading promoters; 2 ) seek reading spaces/reading corners for children and families; and 3) record the use and outcome of the reading spaces (reading homes) service. Happy Reading's role will be to visit and monitor the homes, support the homes in accessing funding, and support literacy development knowledge within the CLs (Happy Reading, 2017c).3.2.3 Cooperation between Supporting Organisations in Society

Cheunwattana (1999) found that rural library services under the government had limited reading materials and were, therefore, less able to support RfP. However, local/civic involvement could bring success as local resources were mobilised leading to institutional cooperation.

Due to the ineffective and bureaucratic management of public and school libraries, over the past decade, successive Thai governments have initiated independent agencies to support reading promotion. TK Park, a unit founded in 2004 under the Prime Minister's Office, is active in promoting the benefits of reading and learning among Thai people. Alongside a prototype library in a modern mall in central Bangkok, it has cooperated with ONBEC to manage prototype school libraries and with ONIE to establish model public libraries in provincial areas (TK Park, 2015).

Subsequently, in 2010 Happy Reading was established to cooperate with stakeholders in rural areas, as mentioned in the previous section. This section also presents the Tambon Administration Organisation and the private sector, which play influential roles in both specific local areas and at the national level.

\subsubsection{Tambon Administration Organisation (TAO)}

The Decentralisation Act of 1999 provided authority and resources to local communities in Thailand, and the Tambon (Sub-District) Administration Organisations (TAOs) were 
established to administer community reading centres that were previously the responsibility of ONIE. Since TAOs are responsible for the welfare of women, children, youth, elderly people, and people with disabilities, child development centres (CDCs) were also transferred to TAOs. Therefore, since 2000 TAOs have played a major role in local education and reading for all age groups.

However, the TAOs' budget was known to be focused primarily on "construction rather than on education/welfare oriented projects" (Rajchagool, n.d. p. 41). Also, each TAO had different capacities to support the integration of fragmented education and public health services, especially for young children. UNICEF (2016) identified that in Thailand there were eight organisations responsible for ECD but there was no linking authority to coordinate them. Happy Reading therefore recently worked as a catalyst in the public health system by promoting the establishment of the ECLF in local communities using the social security budget allocated by the National Health Security Office to the TAOs.

\subsubsection{Private Sector}

PUBAT and other reading organisations, for example, Happy Reading, ONIE and OBEC collaborate with each other and involve private companies seeking to make reading a part of their corporate social responsibility activities. CP Seven Eleven Public Company Limited supports BSPs in many areas of the country, aiming to nurture the love of reading from an early age. Similarly, The Siam Cement Foundation (2017) cooperates with experts in children's literature to select classic picture books from the world and publish them at cheap prices (less than 100 Baht or 4 NZD) and thus encourage book ownership. The project was called "Bring Good Books to Thai Children".

\subsubsection{The Book Market}

Governments in countries where books are affordable for the general public, for example, Sweden, Japan, and Vietnam, have established mechanisms to control book prices. In Vietnam, the socialist state controls book prices, while the Swedish government establishes financial incentives to promote the publishing industry, and decreased the value added tax on books (TK Park, 2009). In Thailand, the unique book distribution system has been a problem for the publishing industry as book selling is based on the consignment system (Yutisri, 2015). An International Publishers Association 
Board Member (International Publishers Association, 2014) gave insights into both distribution and price, the most critical challenges in the Thai publishing industry:

In Thailand, distribution is a problem. Book stores, particularly independents, are feeling the strain, under pressure from a combination of high urban rents and a pricing structure that means their margins are too thin... There is a sharp divide between urban and rural. In cities, where purchasing power is higher, there's a thirst for knowledge and people are increasingly demanding access to information. Habits are changing slower in the countryside, where books remain a luxury item.

A decade ago Makut Ornrudee was invited by the government to consider the specific reading promotion legislation issue and the establishment of an independent authority to support the book industry. He came up with many innovative ideas to assist writers and small publishers, including a state-owned printing house to publish quality books at more affordable prices. The ideas were shelved until being partly revived by the MOC in 2016. However, the National Book Institute, an authority established by MOC in 2017 involving Makut Ornrudee did not have any authority over the book industry nor in national-scale reading promotion (Kongrut, 2012).

The quantitative data presented by Chongsatitwatana (2011) about Nanmeebook, one of the largest publishers in the country, confirmed the problems faced by the book industry in Thailand. It revealed that in 2011 the large chain book store, SE-ED Book Center, had 390 bookstores in the major urban areas of Thailand and in total it had approximately 3500 bookstores. She calculated that at the end of 2011, 1 bookstore in Thailand served 32,953 people.

The most striking figure was that the average price for a book was 168.15 baht (6.70 NZD) while the minimum daily wage in the city area was approximately 215 baht (8.6 NZD) in 2011. The historical minimum daily wage income shown in figure 3.3 explains why the majority of rural villagers, who do not earn cash daily, cannot afford books. Currently in 2018, the minimum daily wage is 325 baht (13 NZD) (Trading Economics, 2018). In rural areas where people generally rely on seasonal cropping work, the daily income is less than that. Thus, books are still generally unaffordable for rural people. In 2016, the PUBAT President stressed that the country's draft National Learning and 
Reading Frameworks should focus on rural areas where he says parents can neither afford to buy books, nor have much access to books (Suchiva, 2016).

Figure 3.3 Thailand minimum daily wage 2008-2018

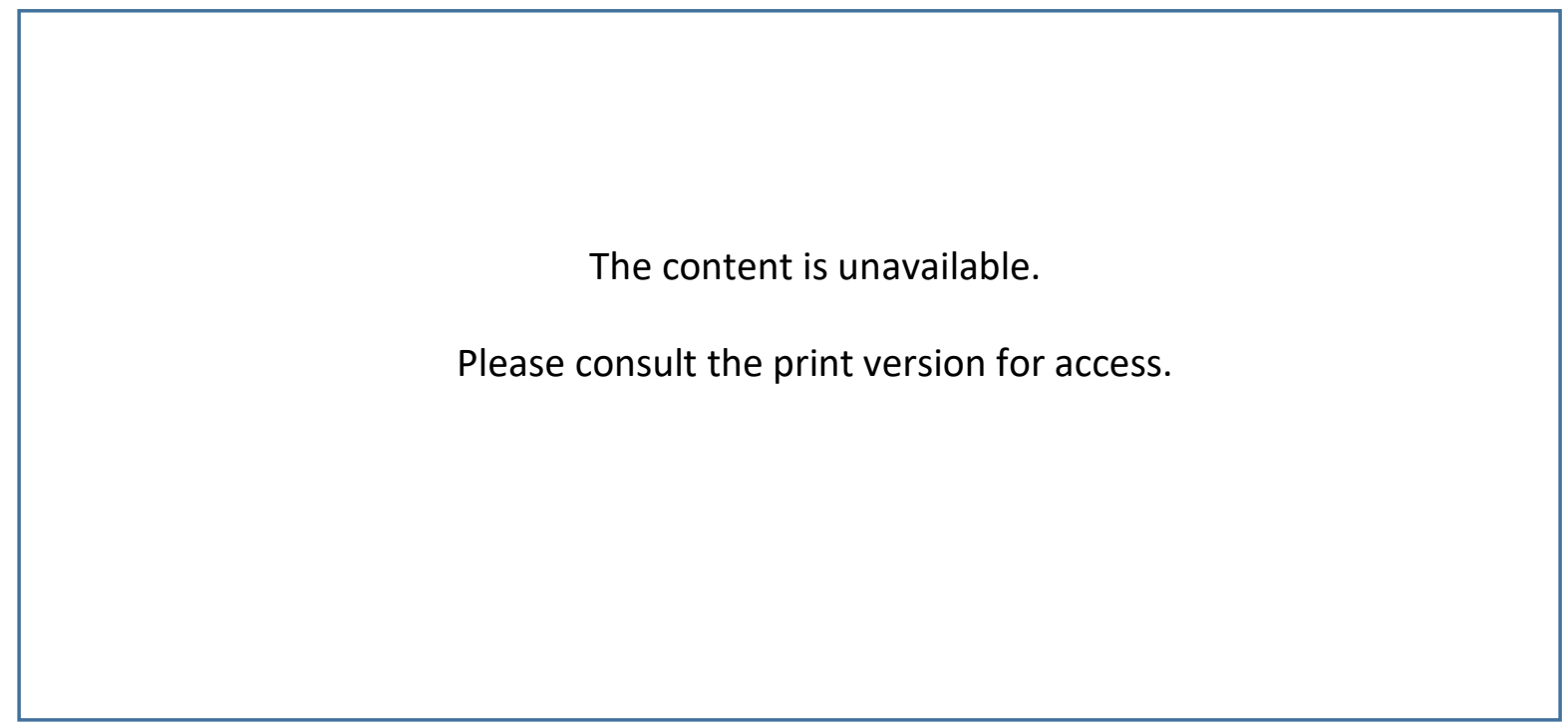

(Source: Trading Economics, 2018)

In all, book distribution, book prices, and book access are interconnected factors hindering RfP. Unequal access to reading materials between urban and rural areas generally limits rural people's literacy development and RfP.

\subsection{Thai Cultural and Social Issues}

A vertical social structure is at the core of Thai society and defines almost every act within it. The structure is firmly rooted at all levels in Thai society and fundamentally affects equality in society. People generally comply with social hierarchy and accept the greater power held by administrators and seniors with a deference that can hinder change (Halinger, 2010). This important dimension tends to contribute to other characteristics affecting RfP practice, for example, income distribution and the didactic and authoritarian roles of parents, teachers and seniors, as well as the prevailing learning tradition.

This section discusses some influential Thai traditions as overarching factors affecting RfP for the whole of Thailand. Next in 3.4 Isan Socioeconomic Influences, specific SES elements that have evolved from Isan's historical and ethnolinguistic development are presented. It also should be noted that contemporary Thai society is confronting a cultural transition, and the extent to which people follow the traditional way of living varies greatly 
between urban and rural areas and between generations (Jirapornkul \& Yolles, 2010). Thus, there is a fluidity of social and cultural elements worth investigating in this study.

\subsubsection{Literacy Traditions}

\subsubsection{Home-school partnerships and Teacher-based learning style}

The ancient tradition that exclusively allows only teachers to teach and transfer knowledge to students means that home-school partnerships are rare in Thai culture. The Thai way of maintaining a harmonious atmosphere and order in school is by emphasising the importance of students' obedience to teachers' instructions. Extreme vertical social relationships focussed on order, formality, and total obedience still exist in Thai education overall (Ekkachai, 2017). In Thai culture, children's attitudes, literacy development, and intellectual development are considered the sole responsibility of teachers because traditionally Thai parents respect the expert role of the teacher (Hallinger, 2010). Deveney (2005) investigated how aspects of Thai culture affected Thai students and stressed that the cultural aspects discourage parents from becoming involved with the school and participating in two-way communication between teachers and students.

As parental involvement is found to be a more powerful factor for RfP than other family background variables (Flouri \& Buchanan, 2004; OECD, 2010), and teachers are only one element in supporting reading (Fletcher, 2017), this hinders mutual interaction between teachers and students and tends to discourage freedom in reading choice and the omission of accountability, both crucial aspects in FVR.

It should be noted that OBEC started to emphasise parental involvement as an important aspect of education reform policies in 2008 (Hallinger \& Lee, 2011). However, Hallinger and Lee (2011) found that without the prior engagement of school staff and adequate training, the cultural transformation and overall education reform progressed slowly.

What makes the Thai case more problematic in terms of RfP is that the teacher-based learning tradition is similar to reading for instruction in schools, which is oriented toward teacher-direction and teacher-ownership (United Kingdom Literacy Association, 2008). 
The alignment of reading promotion with RfP, which is oriented toward child-direction and child-ownership, tends to be a highly contested idea in Thai education (Easeewong, 2011).

\subsubsection{Knowledge-Oriented Mind-set}

A knowledge-oriented mind-set in education is deeply rooted in the Thai learning tradition; reading is normally associated with serious study and knowledge (Ngamwittayaphong, 2011). As mentioned in 3.2.2.2 there was strong evidence from OBEC that reading policy in Thailand focuses on learning and knowledge (OBEC, 2011). Thus, enjoyment, the major component of RfP, has possibly been ignored. TK Park (2008) believed that considering reading as a "living necessity" without RfP is a significant factor contributing to the lower reading rate by Thai people and suggested that the encouragement of RfP by parents and teachers was a strategy to promote reading behaviour.

During 2017-2018, concerns began to be expressed in the Thai government that the knowledge-oriented approach was negatively affecting ECD, parenting, and Thai education. In 2017, the Drafting Committee for Early Childhood Development (2017) contended that a major factor contributing to the low literacy development of children aged 0 - 5 was that many parents and kindergartens with this mind-set inappropriately force young children to learn written alphabet letters and words, and expose them to excessive academic content. This can yield the unexpected result of lifelong low executive function (EF) skills, which ought to be nurtured by free play at this age. The Committee proposed a "Draft Early Childhood Education (ECE) Reform" identifying an urgent need to change existing literacy practices and instead support children to learn naturally by playing and learning from experience using multiple senses.

Consequently, in August 2017, MOE announced the 2017 Early Childhood Curriculum. It identifies age-appropriate suggestions and 23 key experiences in language development. In this curriculum, reading to young children, exposure to picture books, and using role models in reading are clearly stated in the form of eight key reading experiences and three listening experiences (Online Teachers and Educational Professionals Association of Thailand, 2017). 
In late 2017, a respected psychologist and children's rights advocate, Dr. Prasert Palitpholkarnpim, stressed that the government had to find a radical mechanism to change the mind-set at the root of Thai education failure.

\footnotetext{
"We definitely can wait for reading and writing skills until 7, we need not rush. The most important thing to do is that the government must unlock the Thai education focusing on a competitive system... However, the state must support several core practices and structures [for early literacy development], for example, the practice of reading to young children, cheaper picture books, parents' welfare to support them to read for children at least in the first 3 years, and the discouragement of screen technologies before 2 ..." (Thai Tribune, 2017).
}

He mentioned that in the previous 40 years, parents, schools and the Thai education system overall had supported the knowledge-oriented mind-set, and this had resulted in an obsession with competition, tutorial sessions before age 6 , and examinations to enter good schools from grade 1 (age 6).

\subsubsection{Reading to Young Children and Picture Books}

In the West, storytime has been a common practice in public libraries for a century (Bowers, Howard \& Brown, 2018) and Krashen strongly supports the practice as a bridge to FVR, as mentioned in 2.3.4. Storytime is not only used to support literacy development but can also help develop family bonding (McDonald, 2017). However, reading to young children is a rather new practice in Thailand for a variety of reasons. The most important barriers are literacy traditions, and limited knowledge about ECD, as mentioned in previous sections. Another reason Thai caregivers do not read to young children is that they think they are too young to understand language (PUBAT, 2015).

Similarly, the Head of the Child Development Unit of Ramathibodi Hospital pointed out in 2015 that most Thai parents generally do not read to young children as they consider children under the age of 6 are too young. She stressed that parents do not understand that young children enjoy language stimulation from birth. The sooner parents read to their babies and the more books parents read to them, the more receptive babies are to literacy development (MGR Online, 2015). The Bookstart project (BSP) is particularly important for families in poverty as they often cannot afford children's books. For that reason, the paediatrician asked the Department of Public Health (MOPH) to revive BSPs 
to help parents read more to their young children. Her unit found that the BSP motivated $60-70 \%$ of parents to acquire additional picture books, which would not have occurred if the picture books were not initially provided for free (MGR Online, 2015).

An important UN agenda, A World Fit for Children, proposed that there should be a minimum of three children's books in every home that has young children (United Nations, 2002) but the Thailand Multiple Indicator Cluster Survey (MICS) conducted in 2012 and 2016 revealed the disparity of children's book ownership/access at home in Thailand. In the 2016 survey, the percentage and pattern of book access were strikingly similar to that of 2012. Urban children were exposed to more children's books than those living in rural areas and in Isan the presence of children's books in the home was the lowest at 33.8\%, exactly the same percentage as in 2012 (National Statistical Office, 2012; 2016). This mean in terms of children's book access, during 2012-2015 there had been no change both in the whole country and in Isan.

A number of activities that support ECD were added to the MICS 2016 survey. The most important for RfP were the involvement of adults with children reading books or looking at picture books, telling stories, and singing songs, and adults spending time with children naming, counting or drawing things. The most striking disparity lay in household wealth status and the mother's education. Children in the richest wealth quintile were around 3 times more likely to own three or more children's books compared to those in the poorest wealth quintile ( $72.6 \%$ and $22.9 \%$ respectively). This is the same pattern for mothers with no education who are three times less likely to provide children's books for their children compared to mothers with higher education $(15.4 \%$ and $63.4 \%$ respectively).

In all, book ownership and parental factors may contribute to the fact that Isan children aged 3 - 5 had the lowest literacy-numeracy skills in the country in 2012 (59.1\%). In 2016 their skills were higher (67.7\%) than those in the North (62.4\%) but was still around $10 \%$ lower than those in Bangkok (76.0\%) and the Central region (73.5\%).

In the Isan case, a BSP study found that if caregivers are guided and trained, they can perform well and greatly appreciate the results of reading to their young children (Pholkaw, 2009). During caregiver training, the study found that leaders in RfP in rural 
areas need to arrange an event to showcase reading models, pairings of a caregiver and a child who are successful cases of reading to young children, to communicate effectively with rural caregivers (Pholkaw, 2009).

A recent PUBAT study (2015) found that parents and caregivers in Thailand had more knowledge than in the past about the importance of reading to their children aged 0 - 6. However, while young children in Bangkok read and were read to the most (73.8\%), the children of the Northeast read and were read to the least (55.9\%). The pattern of reading within the general population is the same, with those in Bangkok reading the most and those in the Northeast reading the least, both in 2011 and 2013 (National Statistical Office, 2011; 2014).

\subsubsection{Learning Atmosphere}

The learning atmosphere may be the most crucial cultural issue for RfP in Thailand since it relates to the vertical social structure focussed on seniority. Thus, RfP, which needs to be oriented toward child/student ownership (The United Kingdom Literacy Association, 2008) could be a challenge in Thailand. This section presents literature on 1) the desirable low-anxiety situation and the effect of vertical social structure, and 2) sharing reading, an aspect of the learning atmosphere that supports FVR.

\subsubsection{Low-anxiety Situation and Vertical Social Structure}

TK Park (2008) identified the provision of libraries with a pleasant atmosphere as an important environmental factor encouraging reading behaviour. Krashen suggested a low-anxiety situation is the best for nurturing RfP (Krashen, 2011). Ngamwittayaphong's first essential condition for the development of a reading culture in Thailand confirmed Krashen's (2011) ideas. Her study indicated that affective elements, for example, an informal, comfortable, friendly and relaxing atmosphere, and horizontal social relationships are necessary conditions to nurture RfP. Thus, reading promotion, which previously has been predominantly undertaken in school settings in Thailand "will not guarantee the emergence of a reading culture" because the school atmosphere is too formal (Ngamwittayaphong, 2011, p.5). Wech-o-sotsakda (2015) similarly found that formality did not create a comfortable feeling when reading for villagers. In gatherings, 
they preferred not to sit in formal chair and table arrangements but traditionally on mats or low day beds.

\subsubsection{Sharing Reading}

Ngamwittayaphong (2011) identified that in Thailand, group gatherings around written materials bring enjoyment, support and sharing that will unintentionally nurture the love of reading. In Malaysia, Pandian (2011) also found the same positive effect of relaxation among community reading participants.

Book sharing also brings positive effects at the community level. The community library project in eight Islamic mosques in rural Southern Thailand in 2010 led by Makut Ornrudee revealed that as people share books before returning them, books socially bind people and distant communities together, leading to more understanding among people and between communities (Kongrut, 2012).

\subsubsection{Normative Culture and Feminine Society}

Long Term Orientation is a dimension in Hofstede's National Culture dimension that characterises how normative or pragmatic a society is. Hofstede Insights (2018b) explained that:

Every society has to maintain some links with its own past while dealing with the challenges of the present and the future. Societies prioritize these two existential goals differently. Societies who score low on this dimension, for example, prefer to maintain time-honoured traditions and norms while viewing societal change with suspicion. Those with a culture which scores high, on the other hand, take a more pragmatic approach: they encourage thrift and efforts in modern education as a way to prepare for the future. In the business context, this dimension is referred to as "(short-term) normative versus (long-term) pragmatic" (PRA). With a score of 32 on Long Term Orientation as shows in Figure 3.4, the Thai society is a normative one. When comparing Thailand with other Asian countries that have been successful in reading promotion, for example Japan and Vietnam, 32 is rather low when compared to 88 , and 57 respectively. This cultural aspect may contribute to the slow adjustment to new ideas including RfP (Eawseewong, 2011). 
The content is unavailable.

Please consult the print version for access.

(Source: The Hofstede Centre, 2018b)In addition, the current National Council for Peace Order (NCPO) guidelines for education support the normative notion that the aim of Thai education is:

To promote patriotism and national interest among Thai youth. To promote love for the monarchy, pride in Thai history and ancestors and instil a sense of gratitude to the nation, not pushing for the way forward and abandoning the good of the past entirely (Thongnoi, 2014).

This cultural dimension critically relates to RfP; Ngamwittayaphong (2011) identified that reading promoters, as a mechanism to support and stimulate reading, needed to be pragmatic and consider the context within which they work. The pragmatic aspects include the ability to adjust activities, the analytical ability to evaluate the context and situation to design activities appropriate for each community, and the ability to exchange knowledge with others promoters. Successful reading promotion in CLs, thus, cannot be a "one size fit all" model, but should be designed for each local community. Moreover, with the lowest masculinity ranking in Asia (The Hofstede Centre, 2018), Thai society tend not to value material achievement, heroism, and assertiveness. Thailand's 
distinct extreme feminine character (34 on Masculinity score) compared to that of Japan which has a very high Masculinity score of 95, means that Thais prefer to show modesty. Therefore, standing out from the crowd is acceptable or admirable in Japan but not in Thailand. This cultural aspect tends to discourage change and civic participation.3.3.4 Attitudes towards RfP

The everyday use of the term "reading" in discussions of RfP in literate societies can be confusing. Duncan $(2013$, p.5) noted that RfP:

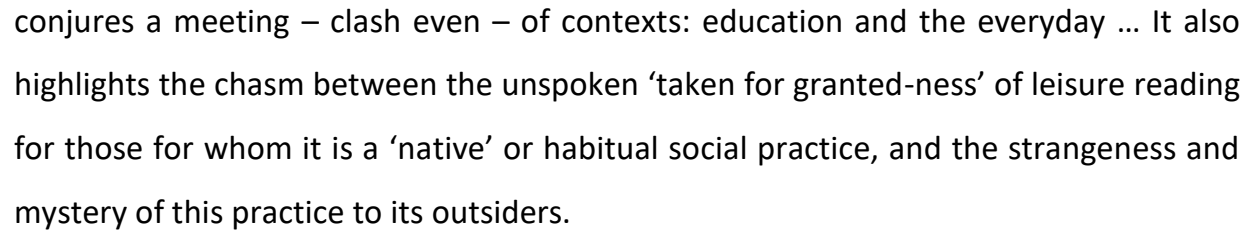

A review of literature from Thailand found that the use of the term is extremely limited. Perhaps there are two dimensions to being an 'RfP native' in the country. The first dimension is the SES of middle class and the lower class Thais. A different SES is characterised by differences in purchasing power, reading behaviour, and library use between the two groups of Thais (TK Park, 2010; PUBAT, 2015), which have been discussed previously. Thus, members of the modern Thai middle class are much more likely to be RfP natives while their rural counterparts are non-natives.

A knowledge-oriented mind-set, the traditional value that associates reading with serious study and knowledge, is another dimension inhibiting RfP in Thailand (Ngamwittayaphong, 2011). On the one hand, RfP natives, for example, reading promotion experts, accept leisure reading as a commonplace concept (TK Park, 2009; Nimkannon, 2006). On the other hand, RfP non-natives such as the Office of the Basic Education Commission (OBEC), the major authority responsible for school libraries (OBEC, 2011), makes no mention of the concept in its solution for the reading problem in Thailand nor in their 2001 Basic Education Curriculum.

As PUBAT (2015) found, the younger generation of Thais do read for pleasure. However, due to low SES, the development of RfP in rural Thailand is likely to be much slower. Therefore, the study needs to explore whether Krashen's ideas hold true in the low SES 
Isan areas. The point of concern will be closely related to Isan identity, Isan language, and the socio-spatial class divide in Thailand.

\subsubsection{Media}

Preference for digital media is an important factor affecting reading behaviour (Dechwittayapon, 2015). Nearly $40 \%$ (37.9\%) of the population prefer watching television than reading for pleasure (National Statistical Office, 2014). More importantly, the survey reported that the majority of the time people spent on reading was devoted to reading social media material (45.5\%). A supporting factor is that smartphone users in Thailand were among the most active in Southeast Asia. In 2016, smartphones were the device that a majority of Thai people used to access the internet (90.4 of respondents) (Kressmann, 2017). Moreover, in 2018 mobile phone subscriptions in Thailand ranked 5th among 137 countries in the world (World Economic Forum, 2018). Greater internet access and cheaper smartphones mean that children are exposed to increased amounts of online media.

Studies found that young children can be particularly negatively affected by exposure to digital media. The Canadian Paediatric Society's Digital Health Task Force (2017) stated that for children aged below 2 , screen time is not recommended, and for children age 2 to 5 , the screen time should be limited to less than 1 hour per day. Similarly, the Drafting Committee for ECD (2017) did not support screen technology for young children as it discourages frontal brain development, and may cause pseudo-autism.

Due to the lack of availability of appropriate digital content, books to support RfP, and media literacy, the excessive exposure to digital media could be a crucial contextual factor affecting RfP in Thai children. A Child Watch project (Promotion of Healthy Lifestyle Programme, 2014) identified that in $2012,91 \%$ of Thai children and youth aged 13-22 had mobiles and 70\% could access the internet via the devices. On average they spent 2 hours 40 minutes per day chatting via mobile/social media. When merging time for web surfing (3 hours), and TV viewing (3 hours), Thai children normally spent 8-9 hours, or half of their waking time on screen-based media. Most importantly, the survey identified that children and youths had extensive access to inappropriate content through the media. In Isan, the difference in access to inappropriate digital content over 
appropriate content was the highest. Moreover, children at the elementary level were the most vulnerable group because they had the most minimal access to appropriate content, but most access to inappropriate.

However, with the provision of appropriate content, digital reading could be an economical choice for RfP. A study of mobile reading in developing countries by UNESCO (2014) found that mobile reading was one tool to compensate for inadequate book access in rural areas in five African counties and two Asian countries studied. The study revealed that people enjoyed reading more when they read via mobile devices. Krashen (2011) suggested that Free Voluntary Web-Surfing (FVS) on the same principle as FVR would encourage literacy development in a second language.

\subsection{Isan Socioeconomic Influences}

Clark and Rumbold (2006) and Clark and Douglas (2011) found that socio-economic backgrounds affected children's RfP. Children from the more privileged social classes read for pleasure more than children from lower classes. In Thailand, the RfP problem is more pronounced in disadvantaged families (both rural and urban-poor areas), where people cannot afford books (TK Park, 2008; 2010; National Statistical Office, 2014). Therefore, socioeconomic status (SES) is a prominent factor affecting book access and RfP in Thailand.

The American Heritage New Dictionary of Cultural Literacy (2005) defines SES as "an individual's or group's position within a hierarchical social structure. Socioeconomic status depends on a combination of variables, including occupation, education, income, wealth, and place of residence." It is normally used by sociologists to predict the behaviour of people in society and in this study it is utilised to understand the reading behaviour of disadvantaged people in the Isan area.

\subsubsection{Poverty, Education and Parents' Migration}

A prominent factor contributing to low SES in Isan is insufficient educational opportunities. Warr (2007) identified that Thailand's poor are overwhelmingly uneducated, rural, and living in large families. In large families, the share of land is gradually reducing due to the division of land by inheritance (Tosakul, 2013). A survey 
on the situation of children and women in Thailand revealed that in Isan, only $69.5 \%$ of children of primary school entry age entered Grade 1 while in Bangkok the figure was 83.2\% (UNICEF, 2016). Therefore, 30\% of children in Isan do not attend primary school. Some of these children, presumably in poverty, will be illiterate for the rest of their lives, and some may start to attend ONIE non-formal education. In fact, the percentage was coincidentally close to that reported in the Reading of the Population Survey by the National Statistical Office (2014) that in 2014, 27.5\% (3.1 million) of the Thai population said that they cannot read.

In addition, in rural Isan, children have generally suffered from limited financial support for education from the government. Cuesta and Madrigal (2014) found that despite a decentralised education policy, education expenditure amongst regions in the country is not "pro-poor" but rather "pro-rich"; thus, "the current decentralised allocation of educational spending is not consistent with an equity-enhancing goal" (p. 239). The outcome of this is that the per student public expenditure on education in the Northeast lags behind other regions, especially Bangkok (Table 3.2).

Table 3.2 Public expenditure on education per student by region, 2008 (Thai baht)

The content is unavailable.

Please consult the print version for access.

(Source: Cuesta \& Madrigal, 2014, p.258)

Isan had the largest number of primary and secondary students, more than one third of the total students in the country. However, at tertiary level, the number drops significantly, as shown in Table 3.3 In 2008, roughly 1 out of 10 students who finished high school in Isan went on to study at university level while in Bangkok, 1 out of 2 students took the opportunity. 
Table 3.3 Total students by region and level, 2008

The content is unavailable.

Please consult the print version for access.

(Source: Cuesta \& Madrigal, 2014, p.257)

To lift social status for minority groups, education plays a major role in Thailand. Setthamalinee (2010) identified that due to their parents' willingness to invest in their education, third generation Chinese Muslims in Northern Thailand enjoy life as middleclass Thais. This also holds true in the case of Isan but only some progressive peasants' families have been able to move up the social ladder. This is because 60 years ago, secondary education was only available in urban areas. After attaining higher levels of education, children or grandchildren of progressive Isan villagers who were "chao ban," common villagers who generally have low education, work as farmers, and live in the village (ban), can lift their status by being professionals, educated, or "chaonai" (Keyes, 2014). Chao ban is a term often equated to the grass roots, the rural, or the "local" (Rakyutidharm, 2014) who generally have low status comparing with "chaonai". The above discussion on educational attainment in Thailand has important implications for the extent to which people can afford books and read for pleasure.

With a low level of education most people, especially in the Isan area, need to work in the agricultural sector or work as unskilled labourers. The seasonal migration to Bangkok of a large casual workforce from Isan has been the norm since the 1970s and Isan people have thus contributed to Thailand's fast growing economy over the decade of 1987-1996 by providing a ready supply of cheap labour (Bird, Hattel, Sasaki \& Attapich, 2011; Warr, 2007). The family structure and children's early literacy development is greatly impacted by this migration. MICS 2016 revealed that in Isan there are larger numbers of children 
who do not live with their biological parents compared to other regions. Only $48.2 \%$ of children in Isan lived with their biological father and $60.6 \%$ with their biological mother. In Bangkok, however, $74.6 \%$ and $86.6 \%$ of children lived with their father and mother respectively. In addition, "The wealth index quintile is positively correlated with the percentage of children living with their biological father or mother" (National Statistical Office, 2016, p.122).

Most rural children are left with elderly relatives, usually their grandparents. Those children left behind possibly have better educational opportunities because of the increased parental wage. However, they generally suffer from the insufficient parenting skills of the caregivers (Jampaklay, Vapattanawon, Tangchonlatip, Richter, Ponpai \& Hayeeteh, 2012). In addition, parents' low SES and parents' migration lead to a decline in children's educational aspirations and attainments (McGregor, Siegel, Ragab \& Juzwiak, 2014). Thus, despite the parents' aspiration to secure additional funds to support their children's education by migrating for work, their migration might paradoxically continue the vicious cycle of poor educational attainment and poverty.

\subsubsection{Isan identity and Isan language}

In general, Thai people are less assertive and less competitive than those in other Asian countries, having the lowest masculinity ranking in Asia (The Hofstede Centre, 2018). This distinctive low success-oriented and success-driven nature of Thai people is more pronounced in the Isan area (Myers, 2005). Generally, low educational attainment and extreme poverty is the cause of low SES and, in the case of Isan, there are additional ethonolinguistic issues including the historical central Thai suppression and the generally lower occupational and social status compared to Bangkok which contributes to the formation of a humble, unconfident, and marginalised Isan identity.

\subsubsection{History}

Isan culture and people has been studied by Keyes (2003; 2014). He asserted that the introduction of primary schools in 1934 , which were not appropriate to the traditional Isan village life, marked "the Thai frame of reference" of national consciousness and loyalty for these isolated minority villagers (Keyes, 2014, p. 63). The educational reforms together with centralised administrative reform and transport reforms enabled Isan 
people to travel to the capital city and made them realise that their future was governed by decisions made in Bangkok. Most importantly, these modern innovations "began to make northeasterners realise that their local culture and patterns of living were considered inferior to those of central Thai" (Keyes, 2014, p. 63). While resistance to central Thai power was evident from the 1920's, mixed feelings and an ambivalent attitude toward their own Isan culture has gradually increased because of the superior attitude of Thai representatives.

Historically, the integration of regions into modern Siam, the rise of elite nationalism, the prevention of the expansion of the Communist Party, development policy, and capitalist industrialisation after 1960 forced millions of Isan to be temporary migrant workers in Bangkok (Keyes, 2014; Wyatt, 2003). The inferior feeling of rural Isan people is generally derived from oppression by Thai authoritarian government officials in their homeland and the attitude of urban Bangkok people who see them as "provincial hicks" (Keyes, 2014, p. 110). Isan villagers humbly identify themselves as "chao ban" and the superior, more powerful, or educated person as "chaonai" (defined in section 3.4.1).

The supremacy of the capital culture of Bangkok and discrimination against Isan people are thus deeply rooted in the Thai mentality, being a norm for both Isan and non-Isan people. A decade ago Myers' (2005) study on Isan identity during 1964-2004, revealed an identity experience of a 30 year-old educated Isan man (born in 1975) who migrated to live as a professional in Australia. Even though his parents were teachers, middle class in Isan society, the participant highlighted the humble nature of Isan people:

\footnotetext{
I had always been taught that the Bangkokian disdain for Isan people is acceptable, that the Thai cultural superiority is a norm, and that Isan people will never reach the status of economic prosperity and social acceptance if they retain their Isanian identities (Myers, 2005, p. 66).
}

Myers (2005) also identified that for decades, Isan people have struggled to seek economic opportunities and have become "the formally-recognized labour class of Thailand," (p. vi). Thus, their social status remains low and the challenge of identity acceptance will continue. McCargo and Hongladarom (2004) also found that Isan identity is "a problematic political construct, reflecting ambiguous self-understandings and self- 
representations." Ethnolinguistic issues, especially identity perception with low selfesteem, may discourage Isan people in terms of CT acquisition and reading, as previously discussed in section 2.3.3. The study will reveal the stigma of embarrassment which accompanies showing Isan identity, specifically spoken Isan language, in formal settings, for example, the school and public reading sphere, which can affect RfP.

\subsubsection{Isan language}

Language plays a prominent role in Isan identity and class division. In the Isan area there are two different classes by ethnolinguistic category. There are "those who had made Thai their first language and those who had stuck with the local language" (McCargo \& Hongladarom, 2004, p. 225). The first group, more fluent with CT, presumably have better education, can have a career outside the agricultural sector and live in the city. They will be able to change from "chao ban" to "chaonai".

As all reading materials are in $\mathrm{CT}$, those who mainly speak their local languages will struggle significantly with literacy, and interactions with formal institutions. To benefit their children, Myers (2005) found that villagers had started to use CT in the family, exclusively when speaking to their children. This will support education attainment which Keyes (1989 p.184) said is "a national system status marker" in Thailand.

As mentioned previously, the use of Isan scripts for traditional education was abolished by the educational reform in the Reign of King Rama V. However, a century later, most Isan people still speak the Isan language in their daily life. Draper (2015) found that at home, in the neighbourhood, and at the markets/shops, the Isan language was used $76 \%, 74.9 \%$, and $64.5 \%$ of the time, respectively. However, it was used less in the workplace, (41.9\% of the time) as well as in tourist attractions $(36.7 \%)$ and the least in school (27.9\%).

As there has not been clear language planning policy in the country, the complex relationships between language, ethnicity, education, and nation-building in Thailand have been an ongoing discussion for scholars, especially in consideration of Thai Lao people in Isan (Draper, 2010; Draper \& Nilaiyaka, 2015; Keyes, 1991; 2003; 2014). Since the Isan language has not been encouraged in school and has no legal status, Isan people tend to link CT with official venues, for example, government offices and libraries. People 
who cannot speak CT clearly tend to be anxious when in contact with officials in those places.

\subsubsection{CT Acquisition for Isan speakers}

Generally, children who speak a local language other than CT will take two years to learn CT before being able to follow lessons (Smalley, 1994). However, Kosonen (2005a, 133) mentioned that "Despite the fact that the use of CT as a medium of instruction is not beneficial for minority groups, the notion that $\mathrm{CT}$ is an uncontested national language is evidently true in the case of the Isan language". This is because Isan people accept that CT is the superior language (Draper, 2010; McCargo \& Hongladarom, 2004). Another two major reasons for the dominance of CT may be that there are too few fluent users of Isan writing (and reading) and there is a lack of multilingual signage (Draper, 2015).

Kosonen (2005a; 2005b) found that language is generally an educational barrier in Thailand. Surveys of educational quality in Thailand identified that children of minorities with poor CT skills had $50 \%$ poorer learning results than CT speaking students in all main subjects; $13 \%$ of Grade 2 students could not read or write CT, and over $25 \%$ of students had problems in reading and writing CT. He concluded that the reason is that teachers and students speak different languages.

UNESCO (2008, p. 104) reported a case study by ONIE of a mother tongue-based literacy project of the Karen hill tribe in Chiang Mai, Thailand noting that "The [Central] Thai language books currently used in schools are designed specifically for [CT] native speakers and are not appropriate for children with other mother tongues". Because of this, it may be assumed that reading can be a difficult activity for those children.

A case in Myers's (2005, p. 66) study portrays the rough way Isan people learn CT. A participant who moved from rural Isan to urban Isan area when he was 12 mentioned that "I was considered the fool of my class for some years before I completely got rid of my Isan accent". In the case that Myers gave, after grade 6 (age 12), the participant needed 5 years to master CT (age 17, grade 11). However, the average length of schooling in Thailand (7.3 years, grade 7 ) will not be enough for Isan people to master $\mathrm{CT}$, the functional literacy tool necessary to deal with reading in general. 
This case in Myers's (2005) study also noted that in urban and educated Isan spheres, in this case, a secondary school in an Isan city, it is better to talk with a non-Isan accent. It is likely that older and lower educated Isan people, who generally have not mastered CT, will not be at ease in their interactions with/in formal institutions in urban areas where they are required to speak CT. Therefore, they can feel intimidated on entering public libraries. This may explain why people with primary education only, and those who work in the agricultural sector, use the village/community library the most (TK Park, 2010).

\subsection{Chapter Summary}

This chapter has provided a review of the literature covering overarching contextual factors affecting RfP: the national reading structure; Thai cultural and social factors; and socioeconomic influences specific to the Isan context.

Generally, Thai people now have a more favourable attitude toward RfP than in the past. This perception may be due to societal changes which are trending away from a traditional interdependent life style to a more self-contained one. However, there remain several cultural and social issues that hinder RfP, for example, the knowledgeoriented mind-set or functional awareness of reading as a "living necessity" without RfP. These contextual factors form the basis of the environment in which the CLs in the study are situated and will help in an analysis and deeper understanding of the cases studied. 


\section{CHAPTER 4 Research Methodology}

This chapter outlines the methodological approach of this research and is split into nine parts. A summary of the key elements of the methodology is presented in Table 4.1.

Table 4.1 Summary of methodology elements

\begin{tabular}{|l|l|}
\hline \multicolumn{1}{|c|}{ Element } & \multicolumn{1}{c|}{ Summary } \\
\hline Paradigm & Social constructivism \\
\hline Approach & Qualitative \\
\hline Method/Design & Multiple case study \\
\hline Data Collection & $\begin{array}{l}\text { 1. Semistructured interviews } \\
\text { 2. Unobtrusive observation } \\
\text { 3. Document analysis }\end{array}$ \\
\hline Data Analysis & Case reports and cross case analysis \\
\hline Unit of Analysis & Type of community libraries (two libraries in each case) \\
\hline Theories & Krashen's Free Voluntary Reading (FVR) \\
\hline & $\begin{array}{l}\text { Asselin \& Doiron's Ecological Framework for Community } \\
\text { Library Developments }\end{array}$ \\
\hline
\end{tabular}

\subsection{Research Paradigm}

A research paradigm is a worldview or philosophical force behind the whole research process. It represents specific views on how studies should be undertaken, analysed and justified. Despite the fact that the paradigm is crucial, and library and information science (LIS) scholars engage in discussions on epistemological assumptions in information sciences journals (Burke, 2007; Talja, Tuominen, \& Savolainen, 2004; Veinot \& Williams, 2012), it is not always a tradition for authors to discuss it in LIS methodology texts (e.g., Connaway \& Powell, 2010; Wallace \& Van Fleet, 2012; Wildemuth, 2009).

Gorman and Clayton (2005) discussed the positivist paradigm and interpretivist paradigms in one paragraph, so Pickard (2013) is the only author who has clearly identified the following research paradigms in LIS, adapted from Lincoln and Guba 
(1985): positivism, post positivism and mixed methods, and interpretivism (empirical interpretivism and critical interpretivism). As this study seeks to understand the phenomenon of RfP in a specific context, it is best aligned with an empirical interpretivist paradigm, or social constructivism.

According to Stake (1995), and Merriam (2009), the construction of knowledge in social constructivism comes from multiple realities rather than a definite objective knowledge. From this epistemological stance, Stake concluded that "there are multiple perspectives or views of the case that need to be represented, but there is no way to establish, beyond contention, the best view" (Stake, 1995, p. 108). Therefore, researchers in this paradigm make sense or interpret others' worldviews. In this respect, the researchers, who act as a research instrument need to position themselves in the research to acknowledge that their cultural background and experience shape the interpretations in the study (Cresswell, 2013). I state my position in section 4.6.1 Subjectivity.

In LIS, one of the early works in the social constructivism paradigm is by Taylor (1968) who contended that people in different professions are socialised into their own world views or their specific information universes because they have similar education and work experience. The paradigm is commonly used to investigate information behaviour, or the information process practice in a specific context (Talja, Tuominen, \& Savolainen, 2005). Therefore, the paradigm suits this study which was conducted in a specific cultural and social context in a region in Thailand, and investigated the worldview and RfP practices of community reading promotors who might not share the same educational background but have rather similar work experiences. Moreover, the study aims to initiate change and/or empower people, which is identified by Merriam (2009) as the purpose of the paradigm.

\subsection{Research Approach}

\subsubsection{Qualitative Research}

In LIS research, Gorman and Clayton (2005, p.8) noted that a "quantitative mode of enquiry is more familiar to most information professionals". However, they argued that qualitative research can help to answer some questions in the profession more deeply 
than a quantitative study and it is more appropriate for the information environment's complexity, the focal emphasis of LIS profession on service, and the social nature of libraries. Stake $(2006$, p.24) also highlighted the different perspectives of professional services and science. Professional services, for example teaching, nursing, and information services, aim to facilitate "people and organizations to function better," whereas science seeks "generalizable knowledge." Therefore, the scientific quantitative approach may not suitable for investigating research questions in professional services. Creswell (2013) identified four common characteristic of qualitative research: 1) the research takes place in a natural setting; 2 ) a key instrument is the researcher; 3 ) it can use multiple data sources; and 4) it allows for a complex reasoning process using both inductive and deductive logic. Another defining characteristic of qualitative research is flexibility (Creswell, 2013; Maxwell, 2013). Gorman and Clayton (2005, p. 69) stressed that "it is fatal to the researcher in this paradigm to follow a set of research patterns". Guba and Lincoln (1994) and Creswell (2013) used the term "emergent design" to describe the flexibility of qualitative study design, which can be altered during the course of the study.

\subsubsection{Trustworthiness}

Since the study has an interpretivist orientation, trustworthiness (Guba, 1981; Zach, 2006) was employed to ensure rigour. Table 4.2 summarised the criteria and methods I used to ensure quality in this research. In Sections 4.4 Selection of Research Sites and Participants, I describe the complex case sampling process to show credibility and transferability. In 4.5 Data Collection I present details of the variety of sources of evidence and variety of data collection techniques used to ensure credibility, dependability, and confirmability. 
Table 4.2 Criteria and methods to ensure quality in qualitative research

\begin{tabular}{|l|l|}
\hline Criteria & Methods to ensure quality in qualitative research \\
\hline $\begin{array}{l}\text { credibility } \\
\text { (plausible findings) }\end{array}$ & $\begin{array}{l}\bullet \text { completeness of the data collection } \\
\text { ucce of multiple analytical perspectives } \\
\text { accuracy of the conclusions }\end{array}$ \\
\hline $\begin{array}{l}\text { transferability } \\
\text { (context-relevant findings) }\end{array}$ & $\begin{array}{l}\bullet \text { use of theoretical and/or purposive sampling } \\
\text { development of a thick description of the data that can be } \\
\text { reviewed by others }\end{array}$ \\
\hline dependability & overlapping methods of data collection \\
\hline confirmability & $\begin{array}{l}\bullet \text { variety of sources for data collection } \\
\text { the researchers' reflexivity practice }\end{array}$ \\
\hline
\end{tabular}

(Source: Guba, 1981; Zach, 2006)

To address the credibility of the study, after I interviewed all participants and my research assistant translated their words to the Central Thai language (see the challenge in 4.7.1 Languages). I sent every participant his/her interview transcription. A number of participants revised and clarified their interview transcripts and the process of translation are described in Phase 1: Organising and Condensing Data of 4.6.2 Data Analysis Phases. The translators were teaching professional who have at least Masters degrees in English/Applied Linguistics. However, because they were not experts in the field, in relation to LIS terminologies and concepts, the accuracy of translations from CT into English also depended on me, researcher-cum-translator. This follows the practice of Ismail (2014) who studied factors affecting the implementation of information literacy education in Malaysian primary schools.

To ensure my interpretation, after drafting case reports, I used the "member checking" technique (Stake, 2006). The reports were sent to a major actor in each case who was asked to review the report for accuracy and possible misrepresentation. 


\subsection{Research Design}

\subsubsection{Case Study Research}

Stake (1995) defined case study research as an investigation and analysis of a single case or multiple cases, intended to capture the complexity of the object of study. Merriam (2009, p. 46) also specified the "particularistic, descriptive and heuristic" characteristic of case study methodology. These definitions focus on the particularity of the case and the complex nature of social phenomena in an interpretivist position.

In an operational definition, Cresswell $(2013$, p. 97) defines case study research as "a qualitative approach in which the investigator explores a real-life, contemporary bounded system (a case) or multiple bounded systems (cases) over time, through detailed, in-depth data collection involving multiple sources of information..., and [which] reports a case description and case themes". Yin (2014) also noted that the unclear division between the phenomenon and the context are the most distinctive characteristics differentiating the case study from other qualitative research.

This study utilised a multiple case study design to explore factors affecting RfP reading promotion practice in four types of CLs (cases) in the Northeast region of Thailand. The design was the most appropriate for the purpose of this study for two reasons.

First, RfP and CLs are two contemporary concepts that the study aims to investigate. Explicitly, RfP is a contemporary activity which is generally opposite to the traditional perception of reading in Thai culture (Eoseewong, 2011). Although the village reading centres were the original form of CLs in Thailand, established more than 40 years ago, the first official CLs were only recently introduced through the 2014 Smart Book Houses Project (ONIE, 2014).

Second, the study aims to identify the factors affecting RfP reading promotion practice and also to explore the effects of those factors in multiple contexts. As reading behaviour and literacy are contextual (Adkins, 2009), RfP practice needs to be studied in context. 


\subsubsection{Case Study Research Paradigms in LIS}

The case study approach can be post-positivist when prior development of rich theoretical propositions plays a significant role in guiding the design, affecting case selection, informing data collection and data analysis. In such practice, logical inference or analytical generalisability from case study to theory can be achieved (Yin, 2014). Yin's emphasis on four criteria related to design quality: construct validity, internal validity, external validity, and reliability, made investigation under his strict guidelines assume a post-positivistic orientation.

In LIS, however, scholars take a considerably different worldview. Pickard (2013), for example, takes a more interpretivist view and suggests that a case study's significant contributions are the exploratory and confirmatory aspects of the specific phenomenon or theory. She points out that "[cross-case analysis for multiple case designs] will allow the reader to make sufficient contextual judgements to transfer the holistic case studies to alternative settings" (p. 108). She also suggests that the emerging themes from previous interviews can inform the next interview. Therefore, data collection in multiple case studies in this paradigm need not be strictly uniform.

Case studies have been widely used in a wide range of specific purposes in LIS research since the 1980s (Pickard, 2013). Blake (2003) found that the use of this method in LIS dissertations had tripled during the two periods of 1975-1979 and 1990-1994. Over the past ten years, it has remained a common research method, especially in information seeking, needs, and behaviour research (Haglund \& Olsson, 2008; Mardis \& Everhart, 2014; Martin, 2006; Zach, 2006). The method allows LIS researchers to discover not only general problems but also other in-depth problems that would have been unlikely to have been identified by quantitative methods alone (Radford \& Kern, 2006).

Hart (2010; 2011) used case study design to study CL services (2010), and dual use school community libraries (2011) in South Africa. In 2012 he then studied outreach programmes of public libraries in rural and urban South Africa, using two case studies to examine the contribution and potential of such programmes to social inclusion (Hart, 2012). 


\subsection{Selection of Research Sites and Participants}

The study seeks to identify factors affecting RfP practices in CLs in the Isan area. Two pilot studies were undertaken in an Isan province. These studies raised issues for the refinement of the data collection plan resulting in more efficient site selection, participant selection and data collection. The pilot study, the criteria for selecting the CLs, and key participants are discussed below.

\subsubsection{Pilot Study}

The two pilot studies assisted in refining the data collection plan. The primary data collection method in the study is interviews with three types of participants, discussed below in 4.4.3. The pilot study was undertaken before data collection in order to highlighted issues of both the data collection process and themes for data collection.

The first major issue of the data collection process is the complexity of 'within case' sampling. In both pilot studies, issues related to the reliance on 'gatekeepers' was addressed. In some cases, the key people I contacted had sensible judgements for identifying the most valuable participants. However, in some CLs in the government authority, the initial gatekeeper linked me to an inappropriate person. Therefore, to gather richer data, I could not rely solely on the gatekeepers. I had to acquire the mobile number of every recommended person in order to contact them later to ascertain their appropriateness for participation in the study.

Second, rural Isan people prefer to do things collectively and made their best effort to welcome me, who they considered their superior guest because of my PhD education and social status as a university lecturer. These traditions, however, created an awkward situation for me, as I was not used to some of the cultural aspects still practiced to a great degree in the two villages I visited. I concluded from this that I needed to understand their collective practice and that I could not control the communication of the gatekeeper nor the eagerness of the villagers. These unexpected events helped me understand the Isan culture more deeply as well as other issues underlying the behaviour of Isan people. I learned to build rapport with friendliness and Isan language (see 4.7.3). 
I realised that I needed to use the Isan language with most participants during the data collection phase.

In addition, emerging data in both pilot studies helped shape themes used in the data collection. After conducting the first pilot study in one $\mathrm{CL}$, I was able to see the reality of reading promotion practice and could better conceptualise the interview protocols. By combining several factors together, unnecessary sub-factors could be eliminated.

\subsubsection{Research Sites Selection}

As this study's primary focus is not on representation, generalisation, and theory generation, I utilised Stake's (2006, p. 23) recommendation to select cases that suit three general criteria: 1) relevancy to the issue being studied 2) diversity across contexts; and 3) better opportunities to learn about complexity and contexts. While diversity across contexts is one criterion to consider, the cases that I could access easily, secure better cooperation from participants or be able to spend more time to observe are also valuable to give the best insight to RfP, as suggested by Gorman and Clayton (2005).

There were seven provinces in the Isan area that the Happy Reading website (http://www.happyreading.in.th/library/) confirmed had: 1) local reading promoters; or 2) a reading promotion network. I then selected libraries based on Krashen's FVR theory which need to: 1) provide access to a great variety of books; and 2) provide at least one service or activity demonstrating that library users have the freedom to select books reflecting their reading tastes (Krashen, 2011).

Cases can be both selected ahead of and evolve during data collection (Miles, Huberman \& Saldana, 2014). During data collection in Thailand between January and April 2016, a purposive sampling technique was applied which allows me to select the most suitable examples that would provide a deep understanding of the issues in the study. I gathered data from 6-10 sites from the seven provinces because a large number of cases can lead to thinner data (Miles, Huberman \& Saldana, 2014: Stakes, 2006).

After initial data was collected in two provinces, Khon Kaen and Kalasin, several key participants recommended further appropriate cases. Thus, I used "reputational case selection" (Miles, Huberman \& Saldana, 2014, p. 32). When I was introduced to further 
appropriate cases, I went to the site to observe their appropriateness. I finally collected data from 11 cases. I then iteratively read the interview data and found that in some cases, data collected was too thin for analysis. Thus, two cases were cut from the study and one case (Case 6.1) became a mini case in case 6. Finally, there were eight cases selected for the study which were clustered into four types or four units of analysis. Details of the selected cases in each province are in Table 5.3.

\subsubsection{Participant Selection}

Miles, Huberman and Saldana (2014) suggested that high complexity within case sampling is desirable for a robust multi-case research study. The careful selection adds confidence to research results. They suggested three major aspects for a within-case sampling process: 1) its nested aspect; 2 ) its theoretically driven aspect; 3 ) its iterative nature. The nested nature means that the process might involve the consideration of access to a wide range of activities, documents, and location in one case. The second aspect means that the selection process should be based on conceptual questions derived from theories (whether from the a priori conceptual framework or emerging as the research evolves). The conceptual question determines events, places, and types of person the researcher should select. The last aspect, the iterative nature, means that sampling is an investigation on that evolves during data collection. Therefore, we can identify additional suitable participants during the data collection process.

In this study, RfP is the issue under investigation. Therefore, participants were selected for their ability to give access to and experiences of a wide range of RfP activities, and documents in a variety of locations bounded by each case. For example, an association leader of CL6 eagerly introduced me to her active project in an urban poor area, therefore I sought more participants from the project which I later called CL6.1, bounded in CL6.

The participants in the study were initially determined by contacting each $\mathrm{CL}$ or the reading promoters in each province to identify three types of persons in the CLs: 1 ) educators/local reading promoters; 2) local leaders/library committee/library coordinators; and 3) library staff/library volunteers. 
Although these three types of persons are normally involved in CL's establishment and operation, as the data collection evolved other suitable stakeholders were selected to participate in the study, as shown in Table 5.4 Details of Selected Participants.

\subsection{Data Collection}

As this study investigates a little-understood phenomenon to identify important factors, Gorman and Clayton (2005) suggested that the data collection techniques need to be observation and in-depth interviewing. The study aimed to get rich data on RfP from the sites, thus, these two interpretive data collection methods were utilised. As multiple sources of data and multiple data collection methods can help case studies to verify an observation or interpretation, I also collected documents for document analysis.

\subsubsection{Interviews}

Semi-structured interviews were conducted with single individuals and lasted one to three hours depending on the knowledge and experience of the interviewees that can provide a picture of the current RfP situation in each $\mathrm{CL}$ and perspectives of factors that support and hinder RfP in this context. The interviews were relatively unstructured, leading to interviewee's insight in RfP practices which greatly varied but provided a deep understanding of specific aspects of RfP in CLs. I spoke the Isan language during the interviews and used an interview protocol (See Appendix C). However, as CLs are diverse in nature, the interviewees were greatly diverse. Therefore, several standard questions, for example, the questions related to reading in a second language and hierarchy in society, did not always yield comprehensive answers. Immediately following the interview, I made an initial transcription of the recording, and wrote memos of emerging themes, as suggested by Pickard (2013). Therefore, I needed to adjust my questions in each interview to illuminate emerging themes. In terms of rapport building, I noted that villagers were not comfortable communicating in a formal atmosphere with chairs and a table. Therefore, for half of the interviews I decided to sit with them on the mat or Thai low daybed and not take notes. 


\subsubsection{Observations}

Unobtrusive observation used in this study aimed to gain a more comprehensive understanding of RfP practice in context. Library visits and library activity attendance were two ways used to observe RfP in CLs. I tried to minimise my intrusion while observing each library in its ordinary activities and context (Stake 2006). However, in the Thai tradition, I could not avoid being a special guest. People who I interviewed or were told that I would observe their CLs often showed up, greeted me, and introduced me to users as a respected scholar guest. I needed to spend a longer time than anticipated at each site until people realised that they did not need to pay special attention to me. During the observations, I investigated the "present orientation" of the behaviour and activities that occurred in CLs (Gorman \& Clayton, 2005, p. 104). Authorised persons responsible for the library or the activity completed consent forms and a notice of consent was put in the library and participants informed at the start of any activities involved in the observation.

\subsubsection{Documentary Analysis}

The documentary analysis aimed to analyse documents directly related to RfP practice (i.e. statistical records of books borrowed) and management policies which might indirectly relate to RfP practice. In addition, documentary sources, including documents published and posted on websites, were also initially used to provide background information on each CL. As the CLs were mostly informal organisations, I rarely found management policies (i.e. yearly plan, service policy, acquisition policy, and collection development policy). I generally found statistical records of books borrowed, which signifies the readers' freedom to choose books. Other documents analysed included ONIE annual reports, websites, newsletters, project proposals and reports submitted to Happy Reading, and Happy Reading public relations publications. In addition, literature on Isan was used to help provide the relevant cultural background. 


\subsection{Data Analysis}

\subsubsection{Subjectivity}

An ontological assumption of the interpretative paradigm is that the researcher and reality are inseparable. However, in order to establish the reliability of the study, researchers need to have "interpretive awareness" or recognise and address the implications of their subjectivity (Hjørland, 2005, p. 140) and use techniques to limit any misinterpretation. Cresswell (2013) and Maxwell (2013) asserted that the case study method is subjective and relies heavily on researchers' previous experiences and their sense making of participants' worldviews. Therefore, they said, it is worthwhile allowing readers to understand the personal experience of gathering the data and the researcher's background.

I am a middle class Isan scholar who speaks CT language at home, and was raised in an urban area in Isan. My parents were from rural Isan villages but were educated, moved to the city, and taught at a teachers' college before I was born. Thus, I have second hand experience of life in rural villages. My undergraduate time at Thammasat, the oldest social science university in Thailand, made me more aware of social justice, and horizontal divides in society. Paradoxically, it was in Bangkok that I sensed the marginalisation of students who were not from the central part of Thailand. I did activities with students from other parts of the country who were also challenged with identity building. The suppression of being "the other" in prestigious Bangkok society was a positive force behind my academic achievement. As I could not find a relevant job in Isan, I worked in Bangkok and furthered my study there for 11 years in total.

I became involved in community services in rural Isan areas while working for the Khon Kean university mobile library project, and the Isan migrated workers' preparation project. Therefore, I have some background knowledge of the Isan rural area but I was a novice to qualitative study. As discussed in 4.4.1, the initial fieldwork experience during the pilot study provided the realisation that I had not been brought up in the traditional Isan culture. Therefore, data collection in CLs helped me understand the Isan culture more deeply in context, as well as other issues underlying the RfP behaviour of Isan people. 


\subsubsection{Data Analysis Phases}

This study aims to extend a clearer understanding of RfP practice in Thai CLs through explanation and description. Although this study primarily had a descriptive and exploratory orientation, prior instruments such as the preliminary research framework and interview protocol were deductively prepared before data collection. If there is a need to identify the analysis approach in the continua of qualitative data analysis, this study uses both inductive and deductive approaches (Braun \& Clarke, 2012).

Creswell (2013) suggested that data analysis is custom-built and is rarely 'off-the-shelf.' Based on Creswell (2013), and Miles, Huberman and Saldana (2014,) the data analysis in this study included the following three phases.

\section{Phase 1: Organising and Condensing Data}

The first phase involved the challenge of managing a massive volume of data and, at the same time, undertaking initial data analysis. Data condensation is the process of making data stronger by "selecting, simplifying, abstraction, and/or transforming the data that appear in the full corpus (body) of written-up field notes, the interview transcripts, documents, and other empirical materials" (Miles, Huberman \& Saldana, 2014, p. 12).

Condensing data was time consuming because all data needed to be read and selected while short memos were generated. Supporting evidence in multiple formats was also identified at this stage. Observation data were rewritten into observational transcripts, and documents found in each CL were selected and labelled with explanations.

After each interview, audio-recorded interviews both in Isan and CT were transcribed into CT by a research assistant. Then I listened to the interview recording and read the transcripts and field note data, and made corrections before interviewing the next participant. During the transcription process, I familiarised myself with the data and started to read and absorb data more deeply. I made observational notes about the potential points of interest and made a short summary of each interview, which was later put in to the project database. After all data were transcribed and corrected for the second time, I sent the transcripts to be verified by the participants. A number of participants revised and clarified their interview transcripts. 
As this stage my interview transcripts were in CT, thus, I condensed them by reading, reviewing, and selecting blocks of data to translate to English. I manually converted them for individual participants into tables and translated half of the transcripts by myself. I sent those extracts to a translator to translate the rest (datails in 4.2.2). I checked all translations to make corrections as needed.

After all data were condensed, most were systematically organised in MS Word format in an Nvivo database. Some files were in pdf and jpeg format. Nvivo software was used in the study to accommodate five functions: 1) storing texts and images in an organised database; 2) coding; 3) data linking; 4) memo editing; 5) data displaying; and 6) literature reviewing.

\section{Phase 2: Categorising Data}

In this study, the smallest unit of aggregated information were "codes" which generated categories (families of codes). Interview transcripts, observational transcripts and documents were openly coded or sorted according to their topics or factors affecting RfP. The initial codes and categories were predetermined by related literature but the analysis was iteratively content-driven. The preliminary set of codes was deductively derived from literature, and the research framework based on: 1) Krashen's Free Voluntary Reading (FVR), and 2) Asselin and Doiron's Ecological Framework for Community Library Developments (The Ecological Framework).

Maxwell (2013) noted that there are three type of categories in data analysis, of which coding, as the first stage of data analysis, is only a form of descriptive or "organizational categories". It can be a critical flaw to synthesise research results at this stage because it is simply "a useful way of ordering your data" (p. 107). Qualitative researchers should consider identifying another two deeper levels: substantive categories; and theoretical categories. The first four initial organisational categories are shown in Figure 4.1 
Figure 4.1 The organisational categories

\begin{tabular}{|c|c|}
\hline A. Contextual Factors & \\
\hline 1. LITERACY & \multirow{17}{*}{$\begin{array}{l}\text { 1. Community participation } \\
\text { 2. Local leaders/educators } \\
\text { 3. Education and training } \\
\text { 4. Responsiveness to local needs } \\
\text { 5. Site selection/location } \\
\text { 6. Budget } \\
\text { 7. Networks/coordination } \\
\text { 8. Library materials } \\
\text { 9. Staffing } \\
\text { 10. Library activities/services }\end{array}$} \\
\hline 1.1 Family Literacy & \\
\hline 1.2 Thai Literacy Policy & \\
\hline 1.3 Isan Language & \\
\hline 1.4 Reading in Second Language & \\
\hline 2. DEVELOPMENT OF A READING CULTURE & \\
\hline 2.1 The Government Reading Policy & \\
\hline 2.2 Cooperation from Organizations in the Society & \\
\hline 2.3 Parents and Teachers & \\
\hline 2.4 Reading Atmosphere & \\
\hline 2.5 Sharing Reading & \\
\hline 3. SOCIAL AND CULTURAL ELEMENTS & \\
\hline 3.1 Vertical Social Structure & \\
\hline 3.2 Collectivist Society & \\
\hline 3.3 Normative Culture & \\
\hline 3.4 Learning Tradition & \\
\hline 3.5 Isan Identity & \\
\hline
\end{tabular}

These set of codes were inductively altered by using the data collected and additional literature in the first round process. The three preliminary categories DEVELOPMENT OF A READING CULTURE, SOCIAL AND CULTURAL ELEMENTS, and CL MANAGEMENT ELEMENTS remained stable.

However, factors under LITERACY which were also included under other categories and Reading in Second Language was found to not be a relevant factor since Isan people study CT as a default language and do not see it as a second language.

After lengthy coding, descriptive codes that refer to the characteristics, and/or roles of individuals dealing with RfP drawn from other categories were consolidated to define the emerging category INDIVIDUAL FACTORS. At this point, I could see that there were two major types of individuals in my study, leaders in RfP who initiate, lead, and mobilise $\mathrm{RfP}$, and reading promoters who undertake RfP activities in communities including family 
members, SOTs and ONIE reading volunteers, teachers, VHVs, youth groups, and CL officers.

These two major groups have their own characteristics. Most importantly in this category, training was stated to be particularly crucial for reading promoters who normally have less formal education than the educators. The name of this category was later changed to KEY INDIVIDUAL in RFP to reflect their roles as shown in Figure 4.2.

Figure 4.2 Substantive categories after data condensation and the first round of coding

\begin{tabular}{|c|c|}
\hline 1. DEVELOPMENT OF A READING CULTURE & 3. KEY INDIVIDUALS in RFP \\
\hline 1.1 Reading Policy (+Thai Literacy Policy) & 3.1 Attitude toward RfP \\
\hline 1.2 Coordination between Organizations & 3.2 Family (Family Literacy included) \\
\hline 1.3 Reading Atmosphere & 3.3 Local leaders/educators \\
\hline & 3.4 Reading skill \\
\hline zz Literacy Development & 3.5 Reading Promoter \\
\hline zz Book Access & 3.6 Education and Training \\
\hline zz Low Anxiety Situation & \\
\hline 2. SOCIAL AND CULTURAL ELEMENTS & 4. CL MANAGEMENT ELEMENTS \\
\hline 2.1 Learning tradition & 4.1 Community Participation \\
\hline 2.2 Other cultural elements & 4.3 Location \\
\hline 2.3 Isan language \& identity & 4.4 Reading Materials \\
\hline 2.4 Media & 4.5 Reading Activities \\
\hline
\end{tabular}

In this initial stage three concepts emerged from the data analysis, but as a research instrument these codes were not really "substantive" because they did not have separate and independent existences. They were: 1) Book Access 2) Literacy Development, and 3) Low Anxiety Situation. I was not sure where to categorise these concepts so at this stage I put them under 1. DEVELOPMENT OF A READING CULTURE with zz marks. 


\section{Phase 3: Finding Theoretical Categories and Themes}

In the second round of coding, or pattern coding, related literature was added and elements in the theories were used as lenses to look at the categories. The coding process involved iteratively reviewing and altering codes in interview transcripts, field notes, observational transcripts, and documents collected and resulted in theoretical categories. They are: 1) CL FACTORS, 2) THAI READING CONTEXT, 3) THAI CULTURAL AND SOCIAL FACTORS, and 4) ISAN SOCIOECONOMIC INFLUENCES. Based on the four theoretical categories shown in Figure 4.3, I wrote case reports (Chapters 6 to 9).

These theoretical categories are the result of coding during which I interpreted the data collected and additional literature on socioeconomic status (SES). I saw that SES involves not only occupation, education, income, wealth, and place of residence, but also identity and language in Isan. Therefore, the major organisational category which was previously labelled 2. SOCIAL AND CULTURAL ELEMENTS in the preliminary framework and first round of coding, was broken in to two categories, THAI CULTURAL AND SOCIAL FACTORS, which refer to the overarching Thai cultural and social issues affecting RfP, and ISAN SOCIOECONOMIC INFLUENCES which refers to the particular influence of Isan low SES. The categorisation added depth to the analysis and enabled me to see clearly that there were multiple views on ISAN SOCIOECONOMIC INFLUENCES. 
Figure 4.3 Theoretical categories

\begin{tabular}{|c|c|}
\hline 1. CL FACTORS & 3. THAI CULTURAL AND SOCIAL FACTORS \\
\hline 1. reading materials & 1. literacy traditions \\
\hline 2. reading activities & 2. learning atmosphere \\
\hline 3. community participation & 3. normative culture and feminine society \\
\hline 4. location & 4. media, etc. \\
\hline 2. THE THAI READING CONTEXT & 4. ISAN SOCIOECONOMIC INFLUENCES \\
\hline 1. literacy problems & 1. education and occupation \\
\hline 2. national reading policy & 2. poverty and migration \\
\hline 3. MOE Policy & 3. Isan identity \\
\hline $\begin{array}{l}\text { 4. coordination between supporting organisations } \\
5 \text {. book market }\end{array}$ & 4. Isan language \\
\hline
\end{tabular}

zz Literacy Development

zz Book Access

zz Low Anxiety Situation

KEY INDIVIDUALS in RFP

I found the pattern that, overall, the national reading policy had not been a major factor affecting RfP in CLs in the study (except for CLs under Happy Reading funding). The unsupportive national context, however, can be seen as a catalyst for the establishment of most independent CLs in the study. Thus, I saw that KEY INDIVIDUALS in RFP is not one of the theoretical categories but a rather higher level of interpretation or theme.

This theme came from the finding that most CLs were individually initiated by community/local educators, who can be any reading leaders in the area, similar to the civic movement for Reading Gardens in Indonesia. These people would actively introduce the RfP concept, support and seek funding, and train reading promoters about RfP. This emerging theme was important and engaged all other substantive categories. Similarly, much data was sorted under Book Access and I knew that Literacy Development touched most of the codes. Therefore, they should be themes not categories. 
The Nvivo codes in Figure 4.4 shows the evolution of my coding process, started on 11/07/2016 with key theoretical elements, for example, Reading Materials, Reading Activities, and Community Participation. Then on 18/10/2016, I started the INDIVIDUAL FACTORS, which I later changed to KEY INDIVIDUALS in RFP.

Later I was able to identify from the data and justify from the literature that "Leaders in RfP" existed in the study. Therefore, I created the node. However, their characteristic: passion, and creativity, had obviously appeared since the start of the coding process on 11/07/2016 in 2. Local leaders/educators under B. CL MANAGEMENT ELEMENTS, as shown in Figure 4.1.

Figure 4.4 Nvivo Codes

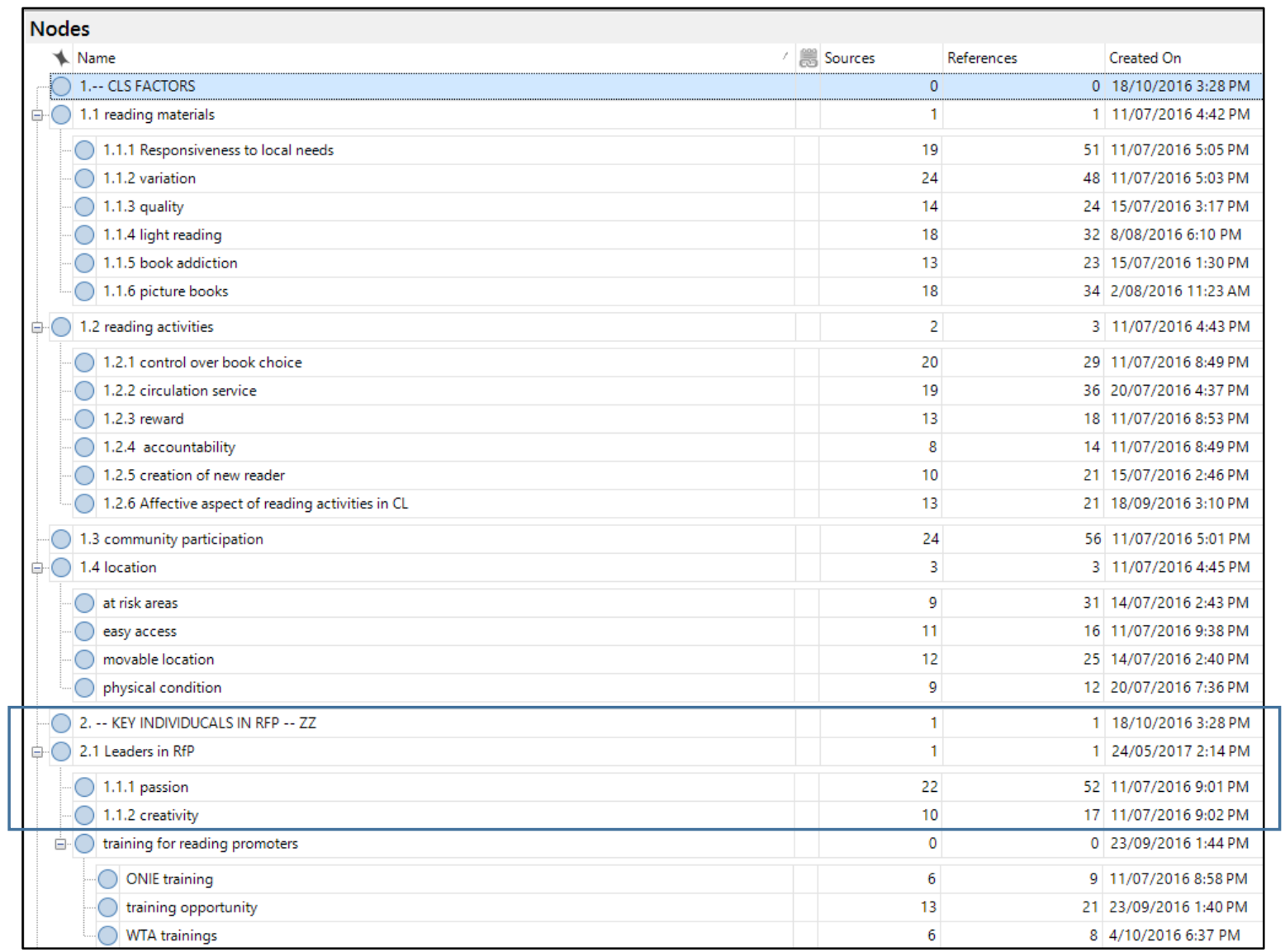




\section{Phase 4: Cross-Case Analysis}

After writing the four case reports, I compared, contrasted, and combined theoretical categories into a synthesised cross-case analysis (Chapter 10). The four themes in this analysis reflect holistic concepts I interpreted from both theory and findings in the study. Most of these are abstract, latent levels of interpretation and were originally codes that I knew were important but did not seem to fit into the coding scheme. I saw that Book Access, Literacy Development, and Low Anxiety Situation are in fact abstract concepts and I conceptualised them as the dimensions reflecting the holistic story of RfP practice in CLs. Figure 4.5 shows the themes.

Figure 4.5 Theoretical Categories and Themes

\begin{tabular}{|c|c|}
\hline \multirow{2}{*}{$\begin{array}{l}\text { 1. Community Library Factors } \\
\text { 1. reading materials } \\
\text { 2. reading activities } \\
\text { 3. community participation } \\
\text { 4. location }\end{array}$} & \multirow{6}{*}{$\begin{array}{l}\text { A. Provision of Book } \\
\text { Access in } \\
\text { Disadvantaged } \\
\text { Areas } \\
\text { B. Literacy } \\
\text { Development } \\
\text { Support } \\
\text { C. Provision of } \\
\text { Inclusive, Safe } \\
\text { Atmosphere } \\
\text { D. Active Role of } \\
\text { Individuals in RfP }\end{array}$} \\
\hline & \\
\hline $\begin{array}{l}\text { 2. Thai Reading Context } \\
\text { 1. literacy problems } \\
\text { 2. national reading policy } \\
\text { 3. MOE Policy } \\
\text { 4. coordination between supporting organisations } \\
\text { 5. book market }\end{array}$ & \\
\hline $\begin{array}{l}\text { 3. Thai Cultural and Social Factors } \\
\text { 1. literacy traditions } \\
\text { 2. learning atmosphere } \\
\text { 3. normative culture and feminine society } \\
\text { 4. media, etc. }\end{array}$ & \\
\hline $\begin{array}{l}\text { 4. Isan Socioeconomic Influences } \\
\text { 1. education and occupation }\end{array}$ & \\
\hline $\begin{array}{l}\text { 2. poverty and migration } \\
\text { 3. Isan identity } \\
\text { 4. Isan language }\end{array}$ & \\
\hline
\end{tabular}




\subsection{Research Issues and Challenges}

In the research, there were issues and challenges in four areas: languages; cases access; participant access; and cross-case analysis.

\subsubsection{Languages}

McCargo and Hongladarom (2004) noted that there are two ethnolinguistic classes in Isan. Despite the fact that I, the researcher, was born in an Isan family, I speak CT as my first language. However, I also am proficient in the Isan language, the language that I learnt through living in the area for more than 20 years. When conducting interviews in rural areas I used the local language. Then my research assistant who has a degree in education and proficient in both Isan and CT translated it into CT to me for checking. For Jonjoubsong's (2008) study in Isan, because she was not from Isan but needed to conduct the interview in the Isan language, she had to have a native speaker to help her with the appropriateness of wording and the preparation of sentences for opening questions and key dialogues. In my case, pilot studies guided my preparation.

\subsubsection{Cases Access}

As I received HEC approval in late January and finished my pilot studies in February, the data collection started in February and lasted until April 2016. A problem I had not anticipated was that in late March and April, there are important celebrations, for example, the traditional Bun Phawet Festivals, and the National Day of Children's Books. The most important festival was Songkran, the Thai New Year, in the mid of April. Therefore, the appointments were difficult for many villagers and ONIE officers as they had to prepare for the celebrations.

\subsubsection{Participant Access}

Since a list of CLs in Thailand is not available, the acquisition of cases to select was multistage. I contacted more than 60 individuals to gather potential cases, and then called each case and each possible participant. As I aimed to interview three people at each site, in several cases the interview dates were postponed many times. The most severe case was the one in the urban poor community where the library officer did not pick up 
my call because it was from someone she did not know. Eventually, I had to ask the library coordinator, the municipal officer, to contact her.

As education is a status marker in Thai society, my superior status as a scholar from a large university in Isan, doing my PhD abroad intimidated several participants. Most Isan people, even the librarian who has a bachelor's degree, felt themselves to be inferior and were suspicious that I would be arrogant and speak in a scholarly language with them. However, I have an empathetic nature and can speak Isan very well, and can adjust to situations; therefore, they felt much more comfortable after the data collection. I also dressed similarly to Isan villagers so that they felt relaxed and I could build rapport.

\subsubsection{Cross-Case Analysis}

If the research involved a group of people with the same education and same experience, the worldview of participants could be rather unvarying, similar to that of Taylor's (1968) early works in the social constructivism paradigm. However, this study primarily includes participants who have quite different education, ages, experience in RfP and careers, as shown in Table 5.4. Thus, I could not compare and analyse cases by putting results in tables and treat each result independently.

On one hand, leaders in RfP, who are well educated, provided a deep understanding of constructs I asked about and elaborated on them in the interview. On the other hand, participants with less education provided the first-hand villagers/people in urban poor areas worldview with the depth that was not available from leaders in RfP. Each of the dimensions presented a construct of the holistic story of the study; the broader Thai context mostly from the leaders in RfP, and specific Isan contexts from both group of participants.

\subsection{Ethical Considerations}

In order to ensure respondents were fully informed about the purpose and use of their data, information sheets were provided to all respondents prior to their participation in the field study and interview sessions. 
The participants signed a consent form to allow their data to be used in the research (Appendices A1-F3). The names of the respondents and libraries were changed in order to ensure anonymity. After the completion of all sessions a summary of the collected data was sent to participants and after each interview, the recording was transcribed and the transcription was sent to the interviewee for checking.

In the data analysis process, the data and the personal identities of individual participants in the study were kept confidential. I carefully supervised staff involved in the procedure to ensure that they were adhering to best practice in protecting the confidentiality of all participants.

Listed below are some practices used to protect the confidentiality of participants: disseminating research findings without disclosing personal identifying information; storing research records securely and limiting access to authorised personnel; removing, disguising, or coding personal identifying information; obtaining written informed consent from the participant to disseminate findings that include photographic/video images or audio voice recordings that might reveal personal identifying information.

Approval from the Victoria University of Wellington's School of Information Management's Human Ethics Committee was obtained prior to the data collection. 


\section{CHAPTER 5 Setting of Case Study Sites}

This chapter outlines the physical characteristics, ethnic, and economic setting of the Northeast (Isan) region, and the context of the eight case study sites.

\subsection{Isan}

This section presents the historical and general context of Isan. Its isolated physical characteristics, ethnicity and language are presented first. The language aspect presented here is mostly relevant to the context of everyday use, which is different from the role of language in reading and Isan identity described in Chapter 2.

\subsubsection{History, Geography, Ethnicity and Language}

Isan is a vast plateau tilted towards the south-east and drains to the Mekong river which divides Thailand and French Indochina. In the past, the rugged hills to the west and the south separated the plateau from other parts of Siam. The region is separated not only geographically from the other parts of the country by geographical characteristics, climates, soils composition, and natural resources but also by Lao and Khmer ethnicities and their culture. Historically, before being ruled by the Siamese kingdom in the 19th Century, most parts of Isan had been ruled by the Lao kingdom of Lan Xang (Lan Sang) and the lower parts had been ruled by the Angkorian Khmer Empire (Keyes, 2014).

In 1892, the threat of French domination of this large Lao-speaking area, the domain of Siam in the northeast, hastened Siamese national integration which resulted in a loss of autonomy for Isan's cities (Keyes, 1989). Under the new nation state of Siam, modern education reforms positively contributed to the independence of Siam, the formation of contemporary Thailand, and educational opportunities for girls and commoners. However, the traditional mode of instruction in the Isan language, using Tham or Lao Tham, Tai Noi and Lao scripts, dominant in both popular and religious education in Isan monastic schools for centuries, was abolished (Tambiah, 1968). Figure 5.1 shows the map of current Thailand where most parts of Isan are adjacent to Laos and Cambodia. The most obvious evidence of closeness to the Lao kingdom is that Vientiane (Viengchan) 
the Laos capital city is 64 kilometres from Udon Thani province. The distance from Bangkok (Krung Thep) and Udon Thani is 475 kilometres (295 miles).

Figure 5.1 Map of Isan

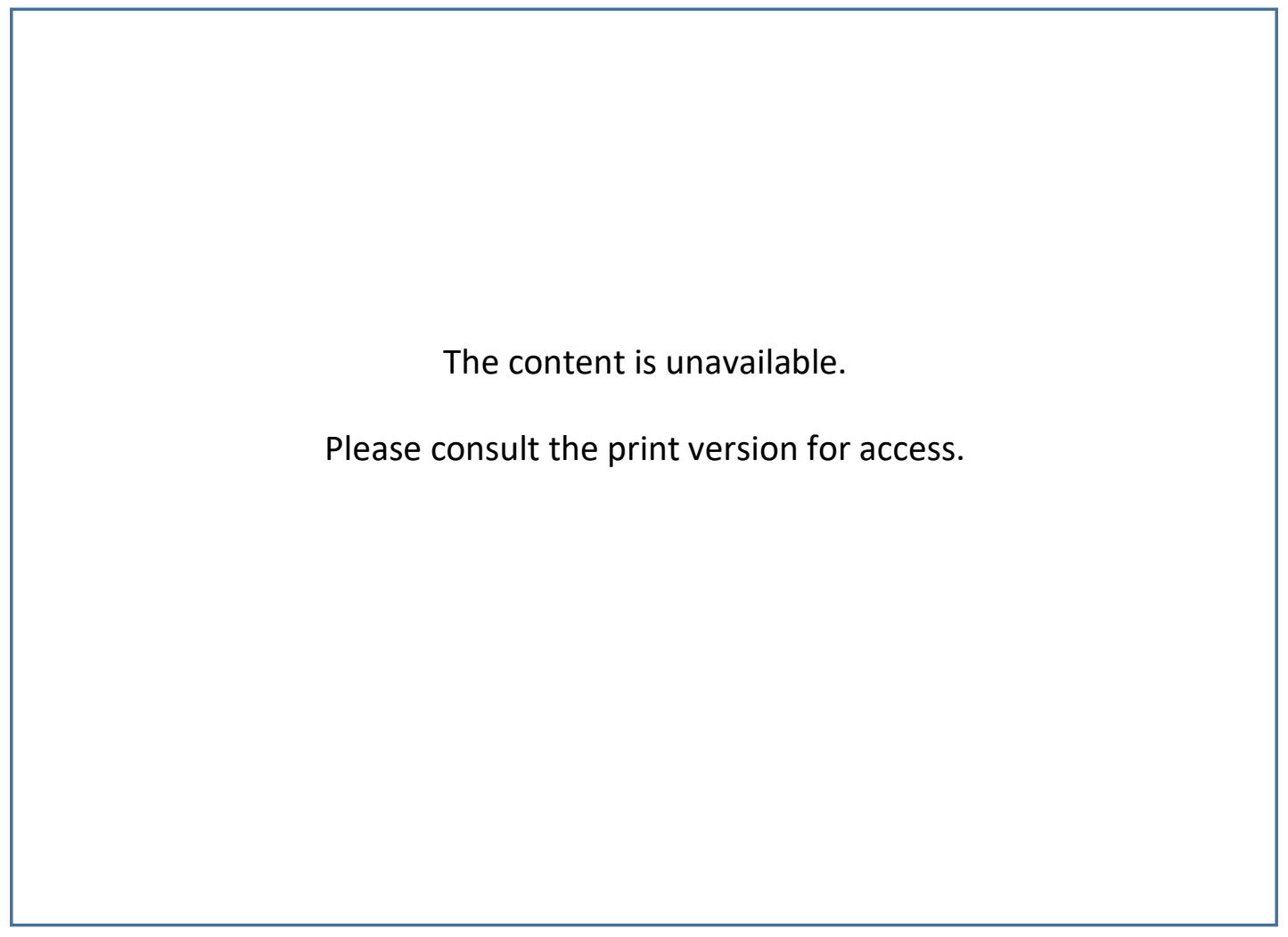

(Source: Brinkhoff, 2018)

Currently, Isan covers 170,226 square kilometre $(66,250$ square miles), roughly half the size of Germany. It is the largest region and is approximately one third of the whole area of Thailand. In all, there are 70 ethnic groups in Thailand, including 24 groups of Tai peoples. Keyes (2014) chose to use languages spoken to represent ethnic composition in Isan. Out of 68.9 million Thai people in 2010, 21.3 million people were Isan inhabitants. The Isan population is relatively homogeneous, approximately $85-90 \%$ speaks languages in the Tai linguistic family, for example Lao Isan, Phuthai, Nyo, and Kaloeng, and approximately $9 \%$ speaks Khmer and Khmer related languages.

\subsubsection{The Economy}

Tosakul's (2013) study of people in two Isan villages demonstrated the precarious nature of the economy. The villagers perceived that "the majority of them just have enough for 
living and eating (p.145)." Most villagers in Isan are small farmers but the breakup of land by inheritance makes the share of land progressively smaller over time. To seek better opportunities, many farmers migrate to work temporarily or permanently in Bangkok and other major cities. Some sell their land to investors from the cities or Bangkok and make themselves landless.

Due to economic disparity, the general Thai public has viewed Isan people as living in poverty, which appears valid according to the 2008 GDP figures in Table 5.1. In 2016, the World Bank Group (2016) reported that poverty in the country was prevalent in rural areas, particularly in the Northeast (Isan), North, and deep South. Therefore, the situation does not appear to have changed over the last decade.

Table 5.1 Regional GDP per capita in Thailand

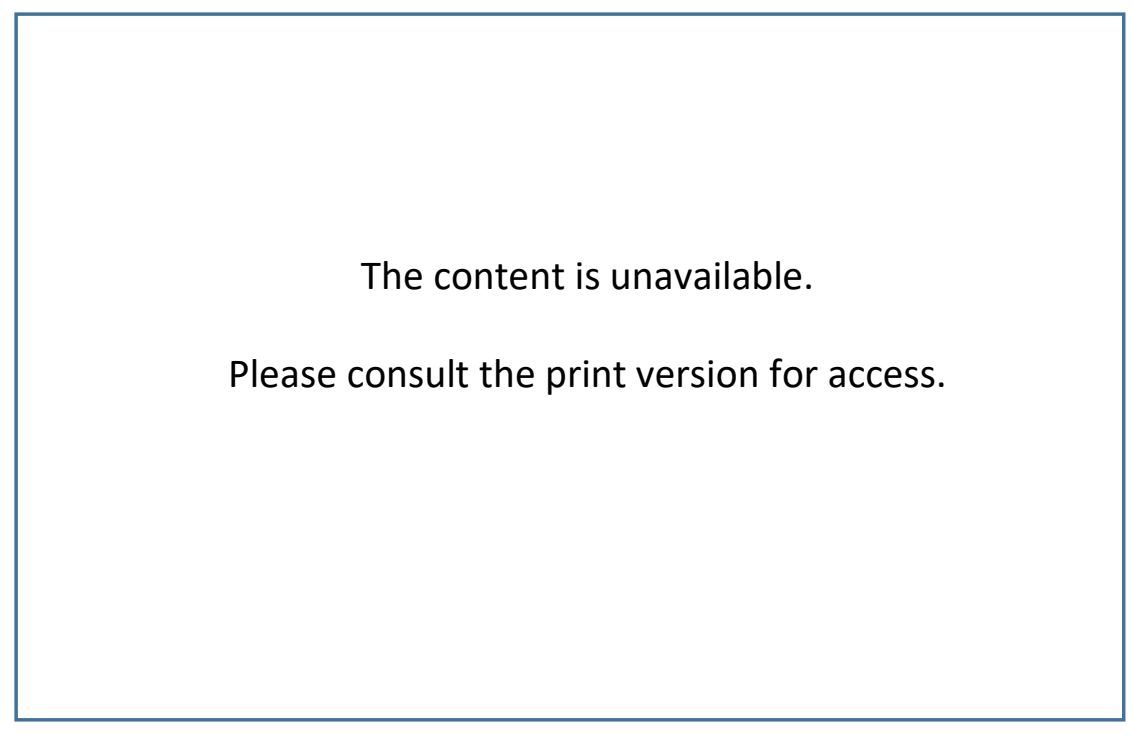

(Source: Tosakul (2013) cited from the Office of the National Economic and

Social Development Board, 2008)

Because of low levels of education, most people in the Isan area are unable to enter professional occupations and need to work in the agricultural sector or work as unskilled labourers in their province, or migrate to work in Bangkok (Bird, Hattel, Sasaki \& Attapich, 2011; Warr, 2007). Figure 5.2 shows the GDP per capita in Thai provinces, identifying that most provinces with the lowest GDP were in Isan. 
Figure 5.2 GDP per capita and population data for the provinces in Isan in 2015

The content is unavailable.

Please consult the print version for access.

(Source: Vanhaleweyk, 2017)

\subsection{Case Study Sites}

Eight case study sites were selected from six provinces in Isan, as discussed in Chapter 4. The six provinces in the study cover most parts of Isan, as shown in Figure 5.2, starting from the upper part, in Loei (1) and Khon Kaen (8) provinces, then Kalasin (9) province in the heart of Isan, and Yasothon (13), Surin (17), and Ubon Ratchathani (19) provinces in lower Isan.

Details of the sites are shown in Table 5.2, and details of selected participants are presented in Table 5.3.

There are four type of case study sites, defined by establishing bodies; 1) An expert organization (Wicha Association - WA), 2) ONIE, 3) health promoters, and 4) individuals. 
Table 5.2 Details of case study sites

\begin{tabular}{|c|c|c|c|c|c|}
\hline $\begin{array}{l}\text { Case } \\
\text { Study }\end{array}$ & Site & Nature & Established by & $\begin{array}{c}\text { Area/Distance } \\
\text { from the city }(\mathrm{km} .)\end{array}$ & $\begin{array}{l}\text { Number } \\
\text { of Books }\end{array}$ \\
\hline \multirow[t]{2}{*}{1} & $\mathrm{CL} 1$ & Children's library & $\begin{array}{l}\text { WA, the abbot, } \\
\text { community leaders }\end{array}$ & rural/26-50 km. & $401-600$ \\
\hline & $\mathrm{CL} 2$ & Children's library & WA, city authority & $\begin{array}{l}\text { urban poor/in the } \\
\text { city }\end{array}$ & $401-600$ \\
\hline \multirow[t]{2}{*}{2} & $\mathrm{CL} 3$ & ONIE SBH & $\begin{array}{l}\text { ONIE, community } \\
\text { leaders }\end{array}$ & $\mathrm{rural} / 5-25 \mathrm{~km}$. & $0-200$ \\
\hline & $\mathrm{CL} 4$ & $\begin{array}{l}\text { Children's library } \\
+\mathrm{SBH}\end{array}$ & $\begin{array}{l}\text { ONIE, the community, } \\
\text { international charity }\end{array}$ & semi-rural/5-25 km. & $201-400$ \\
\hline \multirow[t]{3}{*}{3} & CL5 & $\begin{array}{l}\mathrm{CL} \text { in public health } \\
\text { centre }\end{array}$ & A health promoter & $\begin{array}{l}\text { rural/more than } 50 \\
\mathrm{~km} .\end{array}$ & $0-200$ \\
\hline & CL6 & $\begin{array}{l}\mathrm{CL} \text { in health } \\
\text { foundation }\end{array}$ & $\begin{array}{l}\text { Health communicators, } \\
\text { Happy Reading }\end{array}$ & $\begin{array}{l}\text { semi-urban/ 5-25 } \\
\mathrm{km} \text {. }\end{array}$ & $201-400$ \\
\hline & CL6.1 & $\begin{array}{l}\text { Book collection in } \\
\text { slum community }\end{array}$ & $\begin{array}{l}\text { Health communicator, } \\
\text { community leaders }\end{array}$ & $\begin{array}{l}\text { urban poor/in the } \\
\text { city }\end{array}$ & $0-200$ \\
\hline \multirow[t]{2}{*}{4} & CL7 & Community Library & $\begin{array}{l}\text { An individual (a local } \\
\text { NGO leader) }\end{array}$ & $\begin{array}{l}\text { rural/more than } 50 \\
\mathrm{~km} .\end{array}$ & $201-400$ \\
\hline & CL8 & Children's Library & $\begin{array}{l}\text { An individual (A city } \\
\text { municipal officer) }\end{array}$ & $\begin{array}{l}\text { urban poor/in the } \\
\text { city }\end{array}$ & $0-200$ \\
\hline
\end{tabular}


Table 5.3 Details of selected participants

\begin{tabular}{|c|c|c|c|c|c|}
\hline $\begin{array}{c}\text { Case } \\
\text { /CL }\end{array}$ & Name & Career & Education & $\begin{array}{l}\text { Age } \\
\text { (est.) }\end{array}$ & Role in CLs \\
\hline $1 / \mathrm{CL} 1$ & Anucha & NGO manager & Bachelor's & 50 & leader in RfP \\
\hline $1 / \mathrm{CL} 1$ & Abbot & priest & $\mathrm{PhD}$ & 60 & leader in RfP \\
\hline $1 / \mathrm{CL} 1$ & Karuna & library officer & high school & 50 & library officer \\
\hline $1 / \mathrm{CL} 1$ & Metta & nursery caregiver & high school & 40 & user \\
\hline $1 / \mathrm{CL} 2$ & Makamas & city municipal officer & Bachelor's & 30 & library coordinator \\
\hline $1 / \mathrm{CL} 2$ & Chaiya & city municipal officer & Bachelor's & 40 & library coordinator \\
\hline $1 / \mathrm{CL} 2$ & Orada & $\begin{array}{l}\text { street vendors library } \\
\text { officer }\end{array}$ & junior high school & 30 & library officer \\
\hline $2 / \mathrm{CL} 3$ & Hatai & shop owner, farmer & high school & 60 & $\begin{array}{l}\text { reading volunteer, } \\
\text { community leader }\end{array}$ \\
\hline $2 / \mathrm{CL} 3$ & Lak & district librarian & high school & 40 & library coordinator \\
\hline $2 / \mathrm{CL} 3$ & Thana & $\begin{array}{l}\text { sub-district ONIE } \\
\text { teacher }\end{array}$ & Bachelor's & 50 & reading promotor \\
\hline $2 / \mathrm{CL} 4$ & Tassanee & former ONIE executive & Master's & 70 & leader in RfP \\
\hline $2 / \mathrm{CL} 4$ & Jira & farmer, street vendor & junior high school & 50 & reading volunteer, VHV \\
\hline $2 / \mathrm{CL} 4$ & Kitiya & $\begin{array}{l}\text { sub-district ONIE } \\
\text { teacher }\end{array}$ & Bachelor's & 40 & reading promotor \\
\hline $3 /$ CL5 & Yada & $\begin{array}{l}\text { head of the public } \\
\text { health centre }\end{array}$ & Master's & 50 & leader in RfP \\
\hline $3 / \mathrm{CL5}$ & Nida & farmer & primary & 50 & $\begin{array}{l}\text { reading volunteer, } \\
\text { reading model, VHV }\end{array}$ \\
\hline $3 / \mathrm{CL5}$ & Wanna & primary school teacher & Bachelor's & 50 & reading promotor \\
\hline $3 / \mathrm{CL} 6$ & Naree & $\begin{array}{l}\text { NGO manager, health } \\
\text { communicator }\end{array}$ & Bachelor's & 40 & leader in RfP \\
\hline $3 / \mathrm{CL} 6$ & Nalinee & $\begin{array}{l}\text { NGO founder, nursery } \\
\text { owner }\end{array}$ & junior high school & 50 & $\begin{array}{l}\text { leader in RfP, reading } \\
\text { model }\end{array}$ \\
\hline $3 / \mathrm{CL} 6$ & Orawan & retiree & certificate & 60 & reading volunteer \\
\hline $3 / \mathrm{CL} 6$ & Pichitra & city municipal officer & Bachelor's & 50 & $\begin{array}{l}\text { leader in RfP, community } \\
\text { leader }\end{array}$ \\
\hline $4 / C L 7$ & Pradit & $\begin{array}{l}\text { NGO manager, health } \\
\text { communicator }\end{array}$ & Bachelor's & 40 & leader in RfP \\
\hline $4 / \mathrm{CL} 7$ & Amara & ECD teacher & Bachelor's & 50 & reading promotor \\
\hline $4 / \mathrm{CL} 7$ & Boonta & university student & $\begin{array}{l}\text { high school (doing } \\
\text { Bachelor's) }\end{array}$ & 20 & former reading volunteer \\
\hline 4/CL8 & Aree & $\begin{array}{l}\text { municipal health } \\
\text { officer }\end{array}$ & Bachelor's & 40 & leader in RfP \\
\hline $4 / C L 8$ & Dara & slaughter house officer & certificate & 50 & $\begin{array}{l}\text { former library officer, } \\
\text { VHV }\end{array}$ \\
\hline $4 / C L 8$ & Preecha & primary school teacher & Bachelor's & 25 & former reading volunteer \\
\hline
\end{tabular}




\subsubsection{Case Study 1: CLs established by an Expert Organisation}

CL1 and CL2 shared the establishing body, the expert NGO in early childhood development (ECD). The CLs had large collections of selected books, paid library officers, and generally sufficient training and library management in the establishment of the project. Therefore, they were the best equipped libraries in the study in terms of library elements and library operation. However, the sustainability of the CLs largely depends on the local authorities who provide financial support for the CLs. Political conflicts and policy changes directly affected this type of library.

\subsubsection{CL1}

CL1 was a children's library located in a small district in a major province. The district was rather new as it has been divided from another district in 2007. Therefore, the former local Tambon Administration Organisations (TAO) has been elevated to be a municipality in 2007, even though it was very small and still rural. Most of the population in the district worked in the agricultural sector. It was an area where poverty had been prevalent and the interviews revealed that more than $50 \%$ of the population migrated to work in other places, leaving their children with their parents or cousins.

The library was located in a Buddhist temple where the abbot was an active educator. He had seen for more than 10 years that around half of the children in the area live without financial support from their parents. Because of this situation, he decided to provide educational support for these disadvantaged children. He sought cooperation from many parts of society, established the children's library, and later established a charity school in the temple compound. The abbot provided special access to the CL by opening the temple fence in front of the library to give more access to the library from the main road. The library gate was simple but it was right opposite a local school. The library building was also close to the temple's charity school.

In 2006, the library was initiated by the abbot with cooperation from Wicha Association (WA), an international charity, community leaders of 15 villages, nursery caregivers in child development centres (CDCs), and local youths. TAO allocated the budget to build the library. The WA helped with the design of the building and the book acquisition was 
organised by an international charity. WA is a child-focused, non-governmental and notfor-profit organisation specialising in early literacy development in young children.

The library was well-established and training on reading and child development had been organised for several temporary staff in 2006. The library building was a simple 6 by 6-metre room with two half open walls for easy access. It was airy and well-equipped with bookshelves for children. The outer and inner walls were colourfully painted. The floor was covered with tiles and there were no tables and chairs for users. Users normally sit on the floor and the officer had a working corner with a desk and trolley.

An international charity funded the acquisition of a large collection of selected books for the library on its inception. There were nearly 600 selected picture books, most of which could not be found in shops/stores in Thailand in 2016. Ninety percent of the library materials were picture books which were not normally available in provincial areas. There were also bookshelves for selected books for older children and youth, parenting books, and reference books. The $\mathrm{CL}$ had not received any further grants to acquire more books after establishment.

As picture books are generally not available in rural areas, the $C L$ serves $C D C s$ and families in the district. Half of the books had been lost from the library. When the librarian found lost books at CDCs and primary schools, she saw the books were well-worn. The $\mathrm{CL}$ was the only library in the study that had a permanent library officer. She was hired by the municipality in 2008 though she had never been trained by WA. Her major role was to take care of the library and circulation service. However, due to a local political conflict in early 2017 (shortly after the data collection), the officer informed me that the library was closed and she was moved to work in the municipal office.

\subsubsection{CL2}

CL2 was one of a group of community-based pre-school libraries in an urban poor community in a major city. During the past 40 years, this major city of Isan had experienced rapid changes. The Thai Human Development Index showed that the quality of life in this large province was not good. Out of 20 provinces in Isan, it was ranked 15th for housing and environment, and it was ranked 17th for family and community (Shinseang, 2016). The community was situated in the heart of the city next to the 
government offices where there were more than 100 houses clustered together. People were general wage workers, recycling men, and street vendors. Most of them had little education and had limited chances to improve their lives. Seasonal migration to harvest sugarcane in other provinces had affected their children's education for generations.

The CL was built in 2009 by the WA with a budget from the city municipality. After the WA set up and operated these units for several years, the city municipality ran the mini libraries itself. The $\mathrm{CL}$ was located in the most severely-deprived urban poor community in the city and the library was the only positive space available in the community. Therefore, the library also functioned as an informal CDC.

The $\mathrm{CL}$ was designed by the WA to be a mini, removable, and affordable library. It requires a small space and can contain up to 1,200 books for children and parents. There were around 900 selected books in this $\mathrm{CL}$ and the city municipality regularly budgeted for new book acquisitions. Even though the service provider insisted parents borrow books to take home to read to their young children, they hesitated to as they were aware that they cannot pay for replacements if the books were lost or destroyed. Therefore, few books were lost from the library and children had few chances to be read to by their parents at home.

With support from a Christian school in the city, the main participant, the library officer and her children were offered free educational opportunities, which greatly improved their lives and reading behaviour. The volunteer spirit of the library officer was outstanding and she was well-trained. However, the job of a mini library officer receives low pay and has a yearly contract, so perhaps this is why some mini library officers who were not local people failed to open the CLs in the evening. Consequently, the trusted library officer of $\mathrm{CL} 2$ had become the main person responsible for monitoring the other mini CLs in the area.

\subsubsection{Case Study 2: CLs established by ONIE}

CL3 and CL4 were established by ONIE with the hope that the villagers would be inspired to read more and maintain the CLs themselves. Even though both cases did inspire villagers to read more, there was no sense of self-sustainability at either site, especially in terms of collection maintenance. This type of CL largely depends on volunteer officers 
who are also engaged with other type of voluntary service in their communities, for example VHVs. Management and training emerged as major problems in the ONIE CLs, affecting not only library activities and their sustainability but also the whole RfP practice among rural people who rely on the CLS.

\subsubsection{CL3}

CL3 was one of the CLs under ONIE in a small district in a major province. This CL was a successful Smart Book House (SBH) in 2014 before its name changed to Community Book House. The Village Head who volunteered to give a space at the front of her shop to be a SBH during 2013-2015 was a strong female leader who was trusted by the village women to be the head of the women's empowerment project before becoming the Village Head four years ago.

The village was different from other Isan locations because it was a fertile village with abundant surface water and natural ground water. Therefore, the villagers had more choice of agricultural activities; they could grow rice, sugar cane, tapioca, fruit and flowers. As the village is close to the city, the villagers also worked in factories, and as wage workers and construction workers in the nearby city. Thus, people migrate to work in other provinces less than in the other cases, yet collective community spirit was not strong. The distinctive characteristic of the $\mathrm{CL}$ was the district public librarian (DPL), who not only worked in the district public library, but also worked closely with a sub-district ONIE teacher (SOP) who was directly responsible for ONIE's CL initiatives. The DPL had received several awards for her outstanding work. She holds a degree in Library and Information Science and noticed the villager's interest in the daily newspaper, so she taught them to do clipping, which was not a common practice found in the CLs studied. Clippings were cut from newspapers and classified into three broad topics. This was the evidence presented to show villagers' interest in reading and helped make it an awardwinning SBH.

The interaction between the dedicated DPL and SOPs was also a distinction of the site. Despite criticism from other SOPs that they worked beyond their official responsibilities, the DPL and the SOP were highly dedicated to their work for the CL. 
The CL was a 2 by 2 metres corner at the front of the Village Head's shop. As the collection relied on books and serial publications provided by the ONIE SBH project, there were around 200 books with few children's books. The majority of books were on agriculture, handicraft and income generation, and readers were generally adults.

\subsubsection{CL4}

CL4 was a children's library supported by an international charity and was an awardwinning ONIE SBH in 2015. The CL was in a semi-rural village, $25 \mathrm{~km}$ from a major city. As the distance to the city was short, villagers were a mixture of people who worked in the agricultural sector, service sectors in the city, and government officials.

The village was outstanding in terms of community bonding; this collective aspect had declined in most sites visited in the study. At this site, middle-aged and older people liked to participate in village events collectively and made their best effort to welcome guests. The dedicated Village Head was another factor contributing to the success of the library. He was interested in reading and dedicated his land to build the community centre. The $C L$ was a part of a community centre where the village cooperative and shop was located. The shop keeper also worked as a library officer. The community generally gained support from a former ONIE director who was a prominent reading promoter in the area. The initial success of a Bookstart Project supported by an international charity in the village had later been extended to establish a Family Library.

The Family Library did not include a budget for the library's physical building but a collection of 238 selected picture books, which were both children's books and books for parents. The WA, as the major coordinator of the charity, selected the books. In addition, Happy Reading later supported the acquisition of books for the $\mathrm{CL}$, making it one of the outstanding CLs in the area with more than 500 selected books. The library offered a variety of books both for children and adults including quality donated magazines, for example, the Thai edition of the National Geographic. Books were in good condition and were displayed on bookshelves for children that display book covers face on. The $\mathrm{CL}$ distributed its books to local CDCs and established branches in two other villages. With good relationships between the reading volunteer and SOP in this $\mathrm{CL}$, the villagers and children cooperated and generally enjoyed the activities provided. The topics of 
interest for activities were arts and crafts for children and handicrafts and income generation for villagers.

The case revealed a degree of digital media illiteracy and the business strategies that mobile companies use to attract villagers to go online. The younger generation tend to live independently and read together at the CL less. This is more pronounced when villagers do not consider sustaining the $\mathrm{CL}$ by acquiring new books for the $\mathrm{CL}$.

\subsubsection{Case Study 3: CLs established by Health Promoters}

CL5 and CL6 were established by individual health promoters driven by their personal reading passions and connections. This type of $\mathrm{CL}$ was possibly the most active in the study. However, it needed great effort from the major individuals and also needed financial support from national organisations, for example Happy Reading. In both CLs, training processes and the use of the reading model were outstanding.

\subsubsection{CL5}

CL5 was three library corners at a public health centre in a small province. Its Bookstart Project (BSP), initiated by the director of the centre in 2009 , has been a phenomenon in Thai reading promotion because it was initiated by an individual not an organisation. The director independently established the project without any support from her office, the Ministry of Public Health. Over time, she gained support from the Thailand Research Fund, the local administrative authority and village health volunteers. In addition, the BSP grew bottom up from a sub-district, to district, and then provincial level.

There was influence from the Roman Catholic Diocese, which was established in the lower Isan area in the 1950's. This Catholic community had established its Catholic nursery and primary school during the previous generation. Therefore, in terms of education, this community was different from most rural communities in Isan. After primary school, the church also grants scholarships for villagers to continue their study in Catholic schools in a major the city nearby.

Most people in the village made their living through agriculture and cottage industries, for example weaving and broom making. To seek better job opportunities, migration to other provinces was common in the area. The reading model in this $\mathrm{CL}$ was able to 
support RfP for her two granddaughters because she gained regular financial support from her daughter and her son in law who worked in a hotel in eastern Thailand.

There were three areas for reading in the guest area of the health centre. The first one was the newspaper and magazine corner for people who come to see the doctors. There was also a children's corner, identified by colourful toys with a low fence. There were bookshelves with picture books and children's books. The last corner was a library corner where ONIE previously provided novels and the centre provided books on parenting. The collection of books was around 200 titles, including free distribution picture books for newborn babies.

Free distribution picture books were the most distinctive characteristic of the $\mathrm{CL}$, made possible by a famous picture book author. Nearly 10 years ago, the BSP brought an award-winning picture book author and illustrator to support activities in this province. The author still regularly supports activities and book events organised here. His picture books are the best-selling picture books in Thailand and several have been translated into English, Japanese, Chinese, Spanish, and Portuguese. He wrote several picture books for the BSP and gave their copyright to support the project. Therefore, quality picture books can be printed locally and cheaply (1 NZD per copy). The project does not need to worry about lost books as it distributes free copies of the picture books.

\subsubsection{CL6}

CL6 was a library in a foundation dealing with community health in a province where a large percentage of the population were capable of speaking Khmer. The foundation was in a suburban area of the province. Before the foundation was established, the Director of the foundation opened a nursery, which remains a part of the foundation at present. The main function of the foundation was to operate as a branch of the National Health Security Office Service Centre dealing with health promotion and disease prevention in the province as well as receiving complaints about healthcare provision. The foundation also organises parenting classes to support positive communication with children of all ages. Therefore, the foundation has diverse roles, ranging from early childhood development, sexual health, teenage pregnancy, gender roles, tobacco and alcohol consumption, and food safety. 
Two participants at the $\mathrm{CL}$ were sisters. Although they had different educational levels, they had a progressive understanding of ECD. The unique background of the two interviewees meant that the data collected from the $\mathrm{CL}$ was very rich and led to a mini case under the $\mathrm{CL}$.

The library had been developed from their work as health communicators. Books were one of the tools to promote messages on specific matters. The library's reading activities had been mainly supported by Happy Reading. Although the CL had a large collection of children's books and parenting handbooks, book circulation was mostly done in the library area. Books were taken by the foundation officers to use in activities on the specific health matters they were promoting in remote areas of the province. Languages used were Central Thai and Khmer.

To extend the foundation's reading activities to marginal communities in the city, it created the Book Bank, a sustainable initiative supported by the donated Book Bank Fund, and Community Saving Scheme. Happy Reading also supported the initial project which provided children in at-risk living environments with access to quality children's book, new titles, and a monthly fund for their snacks and related art and craft activities. Starting in 2014, the project "Traveling Book Boxes Project" provided boxes to every urban poor community in the city.

CL6.1 was one example of an active book box project in an urban poor community. The $\mathrm{CL}$ was a mini site under CL6 that demonstrated the efforts of an urban poor community to strive for a better life. People who lived here were a mixture of local people and people who had migrated into the city to seek better job opportunities. The community leader was actively seeking a way to supply new books and resources for reading activities. There was no permanent library space in 2016, therefore they used folding tables and chairs and put them outdoors for reading activities at weekends. Later in early 2017, an extension to the community office had been built to serve as a CL.

In this $\mathrm{CL}$, food and snacks attracted hungry children. Cooking was an important activity linked to reading. Caregivers in the urban poor community had little involvement with the reading activities provided. 


\subsubsection{Case Study 4: CLs established by Individuals}

CL7 and CL8 had been established by passionate individuals who indirectly promoted health and supported youth empowerment in their communities. These CLs largely relied on the individuals who were educated, and well-trained, therefore training was no problem for this type of CL. However, as the CLs informally served as day care centres, they needed both volunteers and paid persons to run the CLs efficiently.

\subsubsection{CL7}

CL7 was located in a mountainous small province. The main crop in the area is sugar cane. Normally Isan rice farmers supplement their income by cutting sugar cane, or as construction labourers, however, in this area, most of them supplement their income by selling lottery tickets in Bangkok and other provinces. Thus, most children in the area need to stay with their grand parents or relatives and many children had insufficient food to eat. The library was operated similarly to a child care centre, and food attracted hungry children who were often ignored by caregivers.

The library was started by a person who has a degree and experience in mass communication and needed to return home to take care of his mother after his father died in 2010. He had worked with children and youth before. Thus he formed a youth club which later became a non-profit organisation for youth development. The project then covered more issues relevant for youth, for example narcotic use and teenage pregnancy.

The library was started from the organisation of the youth club and was located in the community public space. It was located in a renovated deserted village nursery. The renovation turned an old 6 metres by 6 metres building into an inviting library. The floor was newly tiled with light colours and attractive bookshelves were acquired. There were around 500 books and the collection was diverse ranging from picture books, children's books, to translated novels, for example The Gideon Trilogy by Linda Buckley-Archer.

\subsubsection{CL8}

CL8 was a children's library in a food market open until late at night in the city of a medium size province. The library was established in 2008 by a municipal official who 
was concerned that the food sellers' children had nowhere to go while their parents were busy with cooking and selling food until 11 p.m. She sought help from the WA and made library visits to several CLs.

The market was large and there were nearly 50 food stalls though storeholders in the community are normally poor and had limited education so they needed to work at night time. The sellers did not initially understand the advantages that a children's library could bring but they had gradually seen that it helped their children's literacy development, school performance and improved their lives. From a brief observation, people in the community had close relationships. Older children from one family looked after and played with young children from another family. Elementary school children used the library as their playground as the library was the only positive space available for them in the market.

The library had been converted from a communal dish cleaning room. The room was a simple 4 by 4 metres with three walls and an open wall for easy access. It was airy and well-equipped with bookshelves for children. The outer and inner walls were colourfully painted by volunteer students. The floor was covered with light colour tiles and the users normally sat on the floor. There was a television in the CL.

The case had demonstrated strong volunteer spirit since its inception which was uncommon in CLs in Thailand. The case also showed how the founder used her social media connections, youth groups, volunteer officers, and VHVs. Youth groups actively organised many fund raising activities to establish the $\mathrm{CL}$. In the most active period, the library had their radio programme supported by Happy Reading. In addition, several television programmes broadcast their success nationwide. However, in the last three years there had been no volunteer officer in the library so it was not as active as in the past.

Evidence from television programme footage showed that the library was originally well established with a large collection of selected picture books with technical support from the WA. However, after 8 years, the books had been given as rewards in reading activities and a roof leak had destroyed a numbers of books. In addition, the library was an open space and there had been no officer in the library for three years, therefore, a large 
number of picture books had been taken from the library by children and parents. The remaining stock included a large number of handicraft and health books for adults, which had been provided by the city municipality. These books had been added to the library after the municipality realised the civic effort that went into the library's establishment.

\subsection{Chapter Summary}

This chapter presents the broad context of the study and the distinctive characteristics of the Isan region in terms of physical characteristics, ethnicity, language and economy. Tables showing brief details of case study sites, and selected participants are provided before the details of the eight case study sites are given.

The chapter provides the context of the four types of case studies, defined by their establishing bodies: 1) an expert organisation; 2) ONIE; 3) health promoters; and 4) individuals. The detail of each case study site gives an overview of the nature of the site, community context, physical setting, and distinctive characteristics of each $\mathrm{CL}$ under investigation. 


\section{CHAPTER 6 Case Report 1}

\section{CLs Established by an Expert Organisation}

\subsection{Introduction}

In the previous chapter I presented background information about the eight research sites (CL1-CL8), which represent four types or cases of $C L$ under investigation. In this chapter I present the case study findings relating to factors affecting RfP in CLs in the Northeast of Thailand for the first case, CLs established by an expert organisation. The two CLs within the first type of CL were established by an organisation with expertise in early childhood development (ECD): CL1 and CL2.

Both CLs were established with expert knowledge from the Wicha Association (WA). The two CLs, one located in the city and the other in a district, were primarily funded by their local government authorities and aimed to serve disadvantaged children who, in CL1, had been left behind with limited financial support by their migrated parents and, in CL2, lived in an urban poor area with a high proportion of dysfunctional families.

Both CLs had large collections of selected books and paid library officers; they were the most well-equipped libraries in the study overall. However, their sustainability largely depends on the local authorities (TAO and the city municipality) who provide financial support. Thus, political conflicts and policy change can directly affect this type of library.

The case report presents four major categories emerging from the analysis of data as discussed in Chapter 4. They are: 1) the Thai Reading Context, 2) Thai Cultural and Social Factors, 3) Isan Socioeconomic Influences, and 4) CL Factors. Since most CLs in Thailand are managed by the local community and established by organisations with different objectives, contextual factors greatly affect their operation. Therefore, every case report starts with an examination of contextual factors aimed at providing the readers with a solid understanding of the background of each case.

The four participants in CL1 were 1) Anucha- the WA manager, 2) the abbot, 3) Karunathe library officer, and 4) Metta, a nursery caregiver. In CL2 participants were two city 
municipal officers who function as CL coordinators, Makamas and Chaiya, and Oradathe library officer.

\subsection{The Thai Reading Context}

The consideration of the impact of the Thai Reading Context in this case study covers 1) Literacy Problems, 2) the National Reading Policy, 3) the MOE Policy, 4) Coordination Between Supporting Organisations, and 5) the Book Market.

\subsubsection{Literacy Problems}

In Case 1 most research participants knew little about literacy problems in the country except the expert from the WA. He showed his experience and deep understanding of the problems of Free Voluntary Reading (FVR) principles. He pointed out the problem of a vicious cycle created by poor reading skills which comes together with the lack of positive reading behaviour. He noted how avid readers can comprehend text without having to spell out the words so they enjoy reading and the more they enjoy the content, the more they read, hence naturally acquiring reading skills. This implies that books need to be at the appropriate level to suit reading ability, as suggested by Krashen (2014). He mentioned that even though a library may provide some good books, if they are too difficult for some readers, those readers will not visit the library. With this in mind, books were carefully selected by the WA for CL1 and CL2.

\subsubsection{National Reading Policy}

In terms of the government's "Decade of Reading Agenda 2009-2018," described in 3.2.2, leaders in RfP in Case 1 had not noticed any improvement in the country's literacy problems following its implementation. The WA expert's view was that the agenda had simply brought a festive feeling to the public via several campaigns but had not yielded any real change. He believed that the events organised, for example reading contests and storytelling festivals, "are not real practices for reading encouragement" (CL1 Anucha). He contended that the government needs to focus on finding practical solutions to the issues relating to: 1) the book distribution system, and 2) the identification of reading leaders who can encourage new readers. 
The participants in CL2 which was also established before the agenda was launched, had the same experience and added that the policy may reach some groups of educated persons but not rural villagers and people in urban poor area. In this city, successful CLs initiated by the municipality with WA support, had been used by the province to implement reading strategies required by the agenda.

Participants made comments on the methods used to raise public awareness of RfP. A participant mentioned that mass media does not present any positive public figures as role models. In addition, a leader in RfP found that most teachers who attend the training he organises have? a traditional mind-set towards reading and he believed that this is true of teachers generally. Therefore, to create new readers, most participants contended that the central government should not only allocate a higher budget to the CLs and book acquisition but also pay attention to encouraging public awareness and knowledge about books and RfP.

\subsubsection{MOE Policy}

\subsubsection{MOE Literacy Policy}

The MOE literacy policy stating that all children should be literate from grade one (see 3.2.2.2) was considered too demanding by most participants in Case 1. However, they accepted that literacy has a great impact on RfP because if children cannot decipher text, they cannot read for pleasure. The WA expert believes the policy needs to provide guidelines for practice and more detail, for example, the best overall teaching approach, detailed teaching strategies for each stage of literacy, text book use, picture book use, and reading and writing processes. He asserted that "We do not have these details at all" (CL1 Anucha).

\subsubsection{OBEC School Libraries}

As mentioned in 2.6.3, in rural areas, reading encouragement generally does not happen at home and the school library is usually the first reading space for children and school teachers are generally influential figures Case 1 highlights national, local, and personal views on school libraries. The WA expert, who had experience of a city school library project in a low-income area, talked about how school libraries were under resourced 
and why most teachers still have a traditional knowledge-centric mind-set. The books acquired for school libraries are mainly academic and not suitable for either students or the general public for RfP. In his opinion, even if schools permitted people in the community to use hybrid libraries as suggested by Cheunwattana (1999), they would not use them because: 1) the books are not what they are interested in, 2) the books are not categorised well, and 3) the people have poor reading skills.

The Abbot who has a PhD, clearly understood the RfP concept. He mentioned that a major problem in the Thai education system is the inequality in access to high- quality schools in rural areas. This may hinder the development of RfP because high- quality schools are commonly associated with high- quality libraries that support for literacy development and RfP for rural children.

\subsubsection{Coordination Between Supporting Organisations}

CL1 and CL2 had been independently established by TAO and the city municipality, respectively before the Decade of Reading Agenda started. Supporting organisations in Case 1 involved those local administrative authorities, the WA, charity organisations, and the community.

Evidence shows that both CL1 and CL2 were initially influenced by WA training and were run by the WA for the first few years. The WA fully designed the buildings and undertook the book acquisitions. They then offered training on $\mathrm{CL}$ management and reading activities. CL1 was established by the community consisting of the abbot, the village chiefs, the nursery caregivers' network, youth groups, TAO executives, and parents.

Initially, the WA arranged for CL1 to have a large book collection from an international charity that cost around 100,000 baht (4,000 NZD). Later in 2008, TAO allocated a permanent library officer for CL1. However, TAO did not provide the officer with any training during her nine years of service and also did not allocate any additional book budget. The abbot and the library officer both mentioned that the lack of library maintenance negatively affected their library operations and they wished to have further funding for both library acquisitions and building maintenance. 
In $C L 2$, the WA received funding from the municipality to operate small CLs and other libraries in the city. After some years the municipality stopped the contract and those libraries were operated by using municipal officers and hiring people from the community who used to be employed by the WA. However, a municipal officer noted that the city still needs expert knowledge from the WA to help CLs provide a better and a wider variety of activities. The extensive experience of this city municipality was distilled into a municipal report found in CL2 identifying RfP as an approach to encourage the love of reading in the city population. However, the project accorded with the normative Thai belief that knowledge acquisition and life-long learning is important in the modern society. Therefore, RfP was not stressed as an end in itself in the publication.

\subsubsection{Book Market}

As explained in section 3.2.3, the book market in Thailand is a complex system in which books are luxury items for rural people. Most participants in Case 1 agreed that book prices in Thailand are generally high and rural people cannot afford. The WA expert also suggested that the Thai middle class lacks knowledge of RfP which hinders them from buying more books and supporting the book market.

He also argued that the complexity of the children's book market in Thailand impacts not only on book prices but also on the availability of books and bookstores. The lack of knowledge of parents or consumers who do not appreciate that quality picture books are worth the investment, contributes to the low demand and lack of visibility of quality children's books those cost per each item is around 200-500 baht (8-20 NZD) from the market. He explained that “...Many world classics that used to be available 10 years ago have now disappeared from the market. Our [middle class] people do not buy those books because they are not aware of the concept of children books and RfP" (CL 1 Anucha).

He also said there is a small number of bookstores in the country, which contributes to low reading behaviour. Therefore, even when people want books and have the money to purchase them, there might be no books available in their area. He added that this is possibly because there is a low demand for children's books; thus, with the consignment system the bookstores do not want to have them on their shelves for too long. He 
concluded that the main reason for the low demand, especially for children's books, was insufficient knowledge and awareness of literacy development and RfP.

\subsection{Thai Cultural and Social Factors}

\subsubsection{Literacy Traditions}

\subsubsection{Teacher-based Learning Style and Home-School Partnerships}

A teacher-based learning style traditionally does not encourage home-school partnerships, as mentioned in 3.3.1.1. It directly restricts students' freedom in reading choices and RfP in general. It also discourages reading to young children by caregivers at home. Participants in Case 1 suggested that the way children learn and feel toward teachers has changed because of technological advancements that offer more channels for students to acquire knowledge. They agreed that there has been a decline in the dominance of teacher-based learning. Nevertheless, rural people highly respect teachers. The respect for teachers can be seen in CL2. The CL officer, Orada, was respectfully called by the people in urban poor area, Teacher Da even though she does not have full authority similar to the official school teachers.

An educator from a rural area who has been working for the city municipality stated that, in rural areas, parents still believe that their children should learn only from teachers at school. Thus, they think that children aged $0-6$ years do not have to be stimulated in any way at home. In terms of RfP, the WA expert asserted that he found from his lectures for Happy Reading that teachers have limited knowledge about books and most importantly they are not readers, therefore they cannot be tangible models to children

\subsubsection{Knowledge-Oriented Mind-Set}

The knowledge-oriented mind-set, as explained in 3.3.1.2, tends to be deeply rooted in the Thai learning tradition. Most participants in Case 1 agreed that, in general, Thai teachers and educators still have a knowledge-oriented mind-set which hinders RfP, as mentioned by the WA expert. 
The mentality of the Thai teachers is that the kids should read books with knowledge in them...I think reading fiction uses more brain power and concentration, coupled with imagination and other brain functions; more than children who do not read fiction. Moreover, fiction can expose the children to various experiences and a lot of vocabulary (CL 1 Anucha).

In CL2 some policy makers at the city level showed their knowledge-oriented mind-set by questioning the benefit of light reading materials. By contrast, a reading promoter who entirely (and accidentally) understands Krashen's FVR recalled that:

\footnotetext{
Some CLs are given donations of gossip magazines. Some administrators asked that why these books are in the library and how it can help with [literacy] development. We explained that it is what people want to read. It's a good thing that people want to read, right? Perhaps, knowledge is embedded in it. Most importantly, it attracts people to come into the library. When they read the books that they are interested in, they may want to read other more academic books. There are a lot of books waiting for them to read (CL2 Chaiya).
}

A school library report found in CL2 is the most important evidence that the city initiated RfP in its CLs and school library projects. On the back cover of the report, the city mayor stressed that providing children with a great variety of choices and inviting them to read the books they like is more important than focusing on answering academic questions and seeking knowledge from books. He wished to distribute the art of reading to the wider public and make reading as a pleasurable activity for people of all age groups.

\subsubsection{Reading to Young Children}

As mentioned in 3.3.1.3, knowledge about ECD, including reading to young children, is rather new in Thailand. Anucha believed that the gap in the opportunities for middle class and poor children to be read to is huge, and therefore reading promoters need to take the initiative: "Priorities of book reading are different between the rich and the poor. Hence, we need to intervene if we would like to see some changes..." (CL1 Anucha).

In Case 1, all participants who had been directly trained by the WA have sufficient knowledge of reading to young children both in terms of literacy development, positive behaviours, and new reader creation. The Abbot and the library officer in CL1 described 
the natural attraction of picture books held for children and their delight in being read to. Most participants in Case 1 have direct positive experiences of the practice of literacy development and they realised that to support RfP, parents need to read picture books to their young children so that they will acquire basic reading skills and school readiness. After 15 years of experience, the WA expert believed that the WA officers know that there are two conditions that yield good results; 1 ) caregivers need to read regularly and continuously to children (i.e. make it a daily routine), and 2) caregivers need to read a good range of books. In addition, WA officers informally create a dialogue with the community, spend time to talk to the adults in communities, and explain the connections between the books and the children.

The CL2 officer who had been voluntarily responsible for book boxes in the preliminary $\mathrm{CL}$ project demonstrated the positive impacts of access to quality children's books, reading to young children, and parental involvement in early literacy development. She stressed that she gained a huge benefit from having the large picture book collection at home and having the chance to read to her children daily. She could see the significant literacy development in her youngest son who she had read to since he was very young. The boy liked to spell words, read signs, and was able to count when he was 5 .

However, all participants found difficult to make the general public believe that reading to children is beneficial and so easy that any caregiver can do. Some believed that most parents may change their attitudes about reading to young children if they experience the benefits for themselves. In CL2, although the officer found that reading to her young children was valuable, she could not make people in urban poor areas believe the same. She expressed her uncomfortable feeling that people in urban poor areas thought the practice was worthless, and they denied the value of the activity and her efforts. She estimated that only half of the caregivers in the community believe in its value:

I am gossiped about - 'why does she read to young children, the children still do not understand'...It is 50:50; those who believe will read to their children, but those who do not will say that there is no connection between listening, reading, and writing... They do not know about brain function, that it is good to read to children from when they are young (CL2 Orada). 
Moreover, the extreme poverty in the urban poor community obstructs book access and RfP; the people in urban poor area do not borrow children's books from the library because they worry that they cannot pay to replace the books if they are lost or destroyed. Thus, their children have few chances to be read to by their parents. In fact, the city municipality was not concerned about books lost from the library and the library officer confirmed that her youngest and middle children used to borrow a book and hugged it in bed until it was torn. The CL coordinator from the municipality explained:

Parents in the urban poor communities think that books are very valuable so they cannot access them. So, we have to change their attitude because books are provided for them. They definitely can borrow books. Even if they do not return the books, we do not blame them. It is good that they read. They still stick with an old idea that librarians must be really concerned with the books in the library. As they [normally] cannot access books, especially children's books, when they see beautiful picture books, they do not dare to borrow them for home. They are afraid that books will be damaged (CL2 Makamas).

It should be noted that some participants had no knowledge about reading to young children because they had never been trained. Therefore, they asked children to memorise the story, added teaching into the story, and asked children at the end of the storytime what the story was intended to teach. It is a norm in traditional Thai children's books that there is a moral in every story. This didactic mind-set can obstruct RfP.

In contrast, a father who regularly used picture books, instinctively thought that the writer would design picture books well and that adding anything to the story was a distraction. He also found that his sons preferred him to read to them by sitting on his lap rather than watching the same stories on television. The impression of books read by parents tends to stay long-term in children's memory, he believes, because after many years his older son could still remember that he used to listen to the picture books being read to his younger brother. However, due to more work responsibilities both he and his wife now need to work during weekends, and he accepted that he had less time to read to this youngest son than to his first son and he took both sons to the children's library less frequently. In rural areas, most participants also confirmed the same 
situation; that caregivers tend to prioritise their work over spending quality time with their children.

\subsubsection{Low-Anxiety Atmosphere}

A low-anxiety atmosphere is a key factor for encouraging RfP (Krashen, 2014) and Case 1 showed that the WA intended to create CLs that provide a low anxiety situation, thus helping enable Krashen's FRV. The library officer and documents in CL1 suggested that the support provided by the WA was different from the traditional public library and more relevant for serving disadvantaged people. The WA expert pointed out that the $\mathrm{CL}$ that created a low anxiety situation attracted more visitors. He explained the positive effect of a combination of quality new books, attractive buildings, and accessible library officers:

According to my experience, when we open a new library, everyone is excited because all the books are new. Moreover, the building is very inviting, especially if we design the building to be attractive. I think reading is about taste. It is to create taste. Thus, a library's appearance must be nice, look comfortable, and not look like a holy place. When a library is like that, everyone is excited since it has become a new thing in the community. The first reason is that they have never touched these books. The second reason, they have never seen such beautiful [picture] books before, and the third reason is that they have never had any experience of going to a friendly library before... (CL1 Anucha).

Participants generally talked about the comparison between public libraries and CLs in relation to class, showing that middle class and upper class people are generally selfassured so they take their children to the public libraries whenever they have time. Disadvantaged people, however, feel awkward about visiting the formal public libraries. Thus in $\mathrm{CL2}$, it is obvious that a $\mathrm{CL}$ is the answer for this marginalised group, specifically children. Two participants involved with $\mathrm{CL} 2$ confirmed the critical role of the $\mathrm{CL}$ :

The people in urban poor areas do not go to the public library although it is nearby. This is why we have to supply books by building mini CLs in the communities ...Despite problems, we do not remove these CLs from the urban poor community as we feel sympathy for the children. They are very interested in the library...There are always new young children as the birth rate in the urban poor is higher than that in the city... (CL 2 Makamas). 
...I have learned from the statistics that these two libraries [in urban poor communities] out of seven libraries work the best. Not only children use the libraries, but adults also visit them. Thus, for the libraries established by the municipality, it can be concluded that having two libraries in urban poor areas or providing books in these areas is the best way to create opportunities for people in the urban poor areas to access books. ...We need mini CLs for the people who are not in the middle class... (CL2 Chaiya).

A reflection from the library officer- a local urban poor dweller who is taking care of two small CLs added depth to the understanding that CLs in any form provide the only reading opportunities for disadvantaged people. The strong and complex sense of inferiority of people in urban poor area, who feed their families by picking recyclable items from bins in the city, emotionally hindered their public library access. They are conscious of their dirty clothes and their class and feel uncomfortable in the public library space.

In CL 1, the Thai authoritarian tradition was mentioned by the Abbot. He stressed that the library officer has a vital role to create a place that is inviting, child-oriented, and provides choices and freedom:

In terms of library service, the reading atmosphere and the library officer are two key factors affecting RfP. We want to change the authoritarian tradition of ordering children to obey or choosing for them. Forcing children to do anything will restrain them. This leads to non-listening and non-reading habits (CL1 The Abbot).

\subsubsection{Sharing Reading}

As demonstrated in section 3.3.2.2, group gatherings around written materials commonly bring enjoyment in Thailand. Observation at CL1 showed that 6 year-old girls from the temple charity school tried to compete in reading their picture books by following the pictures bringing a fun feeling of competition. In CL2 the youngest son of the $\mathrm{CL}$ officer read a picture book to other children who cannot yet read the books. The boy learned to read by listening to his mother and other children in CL2 when he was young.

In relation to the collective group gatherings previously mentioned, RfP in a collective, deprived society may need to be revisited. In $\mathrm{CL} 2$, the $\mathrm{CL}$ is a positive space not only for children but also for adults with family problems. This evidence shows that people in the 
area who generally live in a traditional collective society enjoy using the $\mathrm{CL}$ as a community centre where they share personal stories, give moral support to each other, and use books collectively.

\subsubsection{Normative Culture and Feminine Society}

It was noted in section 3.3.3 that the normative Thai society may adjust slowly to new ideas and most participants agreed that it is difficult to advocate for new ideas, for example reading to young children. This case also demonstrates the distinctive, extreme feminine character of Thai society which does not approve individuals standing out from the crowd. Participants mentioned that there is often gossip about people who act differently from the majority. The officer in CL2 asserted that normative society is a problem when trying to do things differently. She provided the example of reading to young children as mentioned previously in 6.3.1.3 and also that people in the community gossiped and said sarcastically that she dressed nicely to go to meet her secret lover. In fact, she had to dress appropriately for meetings with the city municipality, for which she was the head of all seven CLs established. Therefore, the officer needed to be patient with the gossip that could have discouraged her involvement with the $\mathrm{CL}$ and practice of reading to young children.

\subsubsection{Attitudes towards RfP}

Evidence from previous sections showed that all participants in Case 1 had positive attitudes towards RfP. Some educated individuals, for example, the Abbot, had a good understanding of Krashen's FVR principles focusing on understandable text, and the benefits of RfP:

RfP is reading that brings happiness. I think happiness comes from the understanding of content and the feeling of enjoyment. If we can't understand the content, it's not enjoyable. In addition, both physical appearance and content of the book can affect the pleasure of reading... The children are happy although they can understand only partially. Just seeing the pictures and listening to the stories, although they could not understand all of it, they like it and they laugh out loud. I think that is it (CL1 The Abbot). 
However, the WA expert said that in general Thais have low awareness of the value of $\mathrm{RfP}$, therefore spending money on books is not a priority in their lives, as mentioned in

\subsection{4.}

\subsubsection{Media}

Most participants talked about the negative effect of IT gadgets, which can distract from RfP. Children and teenagers in urban poor areas whose parents have less time to spend with them can be severely affected by media addiction. The CL officer in CL2 talked not only about people in the community who declined to visit the $\mathrm{CL}$ but also the addiction problem with her own children. Even though her children have good literacy development and do well at school, they showed signs of addiction to electronic media and online games because the $\mathrm{CL}$ officer needed to work at night at a food market and could not be at home with them. The WA expert asserted that being addicted to light reading, which many parents see as junk reading material, is far better than electronic media addiction: "I am among the first people to tell everyone to let the kids read novels or cartoon books. It might sound bad but not as bad as letting the kids get addicted to electronic/digital media" (CL1 Anucha).

Participants also mentioned that high book prices in the country make IT gadgets seem a better investment in terms of reading. However, they believed that modern media cannot enhance children's literacy development as much as reading from the physical books.

\subsubsection{Religions}

Buddhism played an important role in Case 1 because the Abbot was a leader in RfP who led the community and sought academic help from the WA to establish the $\mathrm{CL}$ in the Buddhist temple compound. In Thailand many childcare centres are situated in monasteries as they are accessible public spaces. The Abbot also established a charity school to aid disadvantaged young children. Similarly, in CL2 there was a Christian charity school to provide educational support and a large collection of children's books in the school library, which supports the literacy development of the children from the urban 
poor community who receive support to study there. This can be seen in the life of Orada, the CL2 officer, in the next section.

\subsection{Isan Socioeconomic Influences}

\subsubsection{Education, Poverty, and Migration}

Educational opportunities were mentioned as highly valued among Isan people by most participants. However, most of them said that because of the traditional Thai "face saving" value, village people tend to focus on competing to acquire material goods similar to their neighbours and peers rather than saving money to support education and RfP.

In general, participants noted that rural people with low education often migrate to work in larger cities and leave their small children with the grandparents. In CL1's area, the abbot said the practice was prevalent with approximately $25 \%$ of children not receiving financial support from their parents. The library officer in CL1 confirmed the Abbot's estimation and added that she regretted that these parents were missing the chance to read to their young children. They generally turned to excessive materialism as compensation, which could indirectly hinder RfP, for example through giving their children smartphones and tablets.

All participants strongly agreed that parents' migration radically affected their children's development. The abbot talked about the overall effect and the effect on literacy development coupled with materialism:

\footnotetext{
Parents' migration greatly affects the children in all aspects. Most of these children are slow learners. They need love, care and understanding from their parents. They tend to have complexes [they long for attention] and are spoiled... The children who stay with their grandparents have been greatly affected in terms of literacy development. This is because the grandparents finish only grade 6 , or some only grade 1 . Most of them are almost illiterate (CL1 The Abbot).
}

A nursery caregiver in CL1 noticed that there are significant differences between children who stay with their parents and those live with their grandparents. The former will be well-cared for and be prepared for CDC so they are happier, more ready to learn, and 
can concentrate better. The left-behind children, on the other hand, tend to have problems with literacy development and school life in general. This tends to drive the ongoing cycle of low educational attainment and poverty.

In the cases where poor parents take their children with them for seasonal migration, educational opportunity can end at a very young age. The CL officer's life story in CL2 showed that there are very few chances for people in extreme poverty in Isan to escape the vicious cycle of poverty, seasonal migration, low education, and poor job opportunities. Her life story portrays a profile of a volunteer library officer in CLs whose lack of educational opportunity was due to the economic situation in Isan.

The officer said that when she finished grade 6 , she was offered the opportunity to attend a Christian school for free but when she was in grade 8 (age 14), her parents took her to cut sugarcane with them in the North. When they returned, even though the teacher asked her to resume her education, she chose to work in Bangkok because her parents were in a very difficult financial situation. Her brother also left school at a young age and now has a miserable family life. As the officer believed that education is the key to escape poverty, after marrying she continued her study. Her poor background and the current difficult situation in her urban poor community, however, did not hinder her from RfP and her work at the CL.

\subsubsection{Isan Identity}

Most participants agreed that Isan identity, specifically the Isan language, makes Isan people feel inferior in formal spaces (including public libraries), urban spaces, and Bangkok. Participants also mentioned that Isan people think central region people looked down on them mostly for the fact that they cannot properly speak the Central Thai (CT) language. Educators in case 1 gave their view on rural Isan identity, noting that the people are very humble because they are aware that they are rural people at the base of the vertical society.

To eliminate the Isan identity, language, and confidence problems that parents had encountered, more Isan parents try to have their children speak CT from birth. Participants confirmed that nowadays the Isan spoken language is gradually 
disappearing from the new generation and they are worried that Isan people will have no separate identity in the future.

\subsubsection{Isan Language}

All participants in Case 1 were Isan but there were different opinions on CT acquisition. As an educator, the abbot commented that the policy of using CT as the only means of communication affects the literacy development of children who use local languages at home. He wished that school would start with studying the first mother tongue language then move on to CT and perhaps a third language.

Interviews and observation showed that the important factors which make people easily learn $\mathrm{CT}$ were access to interesting $\mathrm{CT}$ reading material via the school library, reading behaviour, and being read to from a young age. The $\mathrm{CL}$ officers noted that nowadays most Isan children can understand both languages, only some have problems with CT. Picture books greatly help with their literacy development and their language acquisition gradually develops when they follow the pictures in picture books, notice the new words, and compare the Isan language with the CT used in the books.

Another example is from a participant who spoke Isan outside the classroom when she was young but had access to interesting reading material at the primary school library and loved to read from a young age. She explained the acquisition process of the teacher using Isan with them in the early years at school and then starting to use CT when she was in grade 4 or 5 (age 10-11).

However, for participants who had no interest in reading or who could not access CT reading material at their young age, learning and mastering CT could be difficult, even as adults. The $\mathrm{CL}$ officer in CL2 commented that even though she often worked with municipal officers and read picture books to children every day, she still worried about making mistakes when speaking CT.

These different experiences reveal the effect of accessibility of interesting/ understandable reading material in rural/urban poor areas that nurtures both literacy development and reading behaviour. In the situation that a family cannot provide reading materials, school libraries play a crucial role for CT acquisition and RfP. 


\subsection{Community Library Factors}

The main CL factors in the study comprise 1) Reading Materials, 2) Reading Activities, 3) Community Participation, and 4) Location.

\subsubsection{Reading Materials}

\subsubsection{Responsiveness to Local Needs}

In general, participants in Case 1 revealed that responsiveness to local needs was crucial and books and serials were selected for the CLs by considering community context and the reading preferences of local people. Even though the traditional notion of 'knowledge' was mentioned, there was no restriction on light reading, as explained by an administrative officer:

\footnotetext{
The books provided must be the books that the people want to read and also provide knowledge. For example, in Community $\mathrm{Y}$, people who use the library are educated and retired government officials, so they want specific types of books and review magazines. However, some libraries [with a majority of less educated users] ask for gossip magazines, or popular newspapers like Thairat or Daily News (CL2 Chaiya).
}

In $\mathrm{CL} 2$, the WA initially prioritised providing picture books to young children in the urban poor community. However, after there was demand from teenagers and adult readers for romances, a shelf for novels from Thai publishers was provided. Therefore, the library served as a $\mathrm{CL}$ for people of all ages. Although there had been several management problems in the library, the administrative officer contended that the local authority needs to continue the project because the $\mathrm{CL}$ is extremely important for children in the urban poor community.

\subsubsection{Variety and Quality}

Interview and observation data revealed that Case 1 had a strong role to play in the provision of reading materials, specifically a great variety of selected picture books that are not generally available in libraries or provincial markets outside Bangkok. Photos taken at the sites some years ago documented that CL1 and CL2 were well-established with a good variety of quality books. My observation showed that approximately $60 \%$ of stock at both libraries are picture books for young children, and the rest are books for 
older children and youth, including reference books for parents. There are also personal improvement books for young people and adults, and non-fiction translated children's series. The borrowing records initiated by the WA showed the popularity and variety of books that people in different age groups choose to read for pleasure; CL1 is the only library in the study to have such a record.

However, after nine years, the observation in CL1 revealed that half the books had been lost (approximately 400 titles), even though the library has a proper circulation system with borrowing cards. Some lost books were found in pieces in nearby nurseries, which signified heavy use. The decreased number of books in CL1, and lack of new acquisitions, negatively affected library operations to the point that library visits had declined over time.

\subsubsection{Picture Books}

The case revealed that the WA carefully selected children's book for both CLs, and rural people were attracted by the appearance and quality of those books. The abbot and the library officer in CL1 recalled the pleasure that children and adults derived from picture books:

What impresses me most about our library service is the children's fascination when they see our selected picture books. What a beautiful book! They love to see nice illustrations with simple words, which they cannot find in text books. These illustrations ignite their imagination and nurture their literacy development. (CL1 Abbot).

Reading materials are very important, especially in rural areas where books like these are very rare. The children would not have a chance to enjoy these picture books if we ask their parents to buy them. (CL 1 Karuna).

Archival photos showed an active period of a mobile reading promotion activity that the WA organised with CL2 in the area in 2009 portraying rural children's delighted faces when they touched picture books and the unconscious "lost in the story" faces when they were read to by the mobile library officer.

\subsubsection{Light Reading}

As mentioned previously, the WA expert and most of the participants had a positive attitude towards RfP, viewing light reading as a conduit to more demanding reading. This 
section presents additional information on two specific genres, non-fiction (educational) comics and fiction. In CL1, borrowing records showed that non-fiction, for example Korean, Japanese, and Thai educational comics for children were very popular. Examples of content were biographies of world figures, Thai historical figures, body parts/anatomy, survival methods, and mathematics.

Participants saw that these comics contributed to the positive attitude of parents toward comics. The most popular comics found were "The Stingy Family", a translated Korean series. However, opinion about Japanese manga was diverse, some participants did not approve of them being in the CL but most recognised that all types of comics are eligible for RfP. The most important characteristic mentioned about comics was that they fed the imagination more than other type of books. A participant mentioned the effect of battle comics on imagination ability which would support medical and architectural studies.

The WA expert asserted that an addiction to books is crucial for reading behaviour, reading skill, and concentration. He asserted that the library plays a major role in providing any light or hard reading materials that make children read with enjoyment:

\footnotetext{
If a child has read a book and was hooked by the book, the child will enjoy reading and this causes learning momentum. [These children] read young adult books ever since they were in the first grade. We are amazed by their ability" and "In library A, the second grade students [age 8] read 'Five Hundred Lives of the Lord Buddha' which is relatively thick. These children have concentration power and language skill to absorb and enjoy the content of the books. They super enjoy it or we can say they are addicted to books! Thus, I believe every child can do well if there are people working in the library to support them. (CL1 Anucha).
}

The provision of a variety of reading material, both light and hard material that will suit different reading abilities and reading interest, allies with Krashen's assertion about library provision for children in poverty (2014) and also his FRV principle that readers need to be "hooked" and "lose" themselves in the book. 


\subsubsection{Reading Activities}

Content in this section is interwoven with section 6.3.2 Learning Atmosphere, emphasising that a low-anxiety atmosphere encourages the will to learn and read voluntarily. In this section, additional aspects of reading activities relating to control over book choice, the affective aspect of reading activities in CLs, circulation services, reward and accountability, and the creation of new readers are presented.

In accordance with the light reading principle, all participants agreed that giving individuals the chance to choose books they find "interesting" is crucial for reading activity that generates RfP. The WA expert asserted that Thais have insufficient knowledge of the benefit of non-academic books.

All participants in CL1 also agreed that the affective aspect, for example, feeling proud, happy, relaxed, and successful is crucial for reading activities in CL. It is particularly important for young children, who do not know what reading is and will be impressed and introduced to the world of reading by those positive feelings. Arts and craft activities were used to engage children to reading materials in both CLs.

In CL1, with its proper circulation system, the borrowing cards showed that books were regularly circulated, at least until they disappeared from the library. The borrowing cards from books that were lost showed that most of them were popular and had been borrowed more than 10 times. Therefore, as long as there was evidence that books from CL1 were heavily used, it seemed that participants did not worry much about losses. However, the situation was absolutely different in CL2 where the target parents in the urban poor area paradoxically did not dare to borrow books, even when the $\mathrm{CL}, \mathrm{WA}$, and the municipality told them to borrow with no need to return. The officer confirmed that the urban poor parents were very cautious about paying for books. This marks a difference between the two CLs in Case 1.

Participants in Case 1 had a rather neutral view about rewards, perhaps because the WA is strongly focused on the intrinsically rewarding feelings that readers derive from picture books and selected book reading. Neither CLs was in a school context, nor did they need to show accountability for their libraries' activities. 
The most important idea on reading activity found in this Case is the WA expert's view on the priority of the creation of new readers rather than the creation of a new library. After 15 years of establishing approximately 30 libraries around the country, the WA expert found that the ratio of the population who used the library declined after a few years in most areas. He thought libraries have two functions: 1) To serve existing readers (both those who are self-starters and encouraged by the library) and 2) To attract new readers. He asserted that reading promotions which include primary and secondary schools in Thailand need to prioritise developing a procedure to encourage new readers. He was concerned most about the investment in poor children who do not have sufficient and regular opportunities to be read to, compared with middle class children. He found the difference in reading to children between the rich and the poor and questioned if there is a class divide in literacy development: "The question is whether the poor children can have any opportunity to be read to 300 times [before going to school] or not... In this respect, what can a poor kid do? Hence, if we do not invest in generating new readers, we will never take the children anywhere" (CL1 Anucha).

\subsubsection{Community Participation}

As mentioned in 6.2.4, over the last 10 years the abbot and the community have established CL1, thus community participation was strong at this site. The CL officer confirmed the effort resulting in "...people in the community come to use the library services. There are no picture books at the child centres... In this area, children's books are only available in our library. Thus the child centres have borrowed a lot of our books" (CL1 Karuna).

CL2 was also initially established through the volunteer spirit of people in the community who allowed the WA to set up shelves in their houses to serve community members. Beyond the availability of reading material, the WA expert explained that an understanding of reading activities is important: "Book Houses cannot exist by themselves. The people in the community, the adults in the community must have the basic idea of how to create reading activities with children. They need to know what the characteristics and nature of reading activities are" (CL1 Anucha). Therefore, training is needed. 
The municipality was also concerned that the $\mathrm{CL}$ needs community participation. When the library was established, the authority first looked for the people in the community to work with because:

\footnotetext{
...they are the owners of the community and can communicate with people in the community... They have to take care of the library like they are the owners. But, it is difficult to find the people in the community. There is only Orada and her son who are the active people in the community. Another person lives quite far away. The rest are people living in neighbouring communities (CL2 Makamas).
}

All participants agreed that a sense of belonging can sustain CLs, and it is preferable that the library service is cared for by community members who are employed by the local administrative organisations.

\subsubsection{Location}

All participants agreed that location is important for the $\mathrm{CL}$ as it must be easy to access and the users should be able to sit and read at any time. As Thais traditionally sit on the floor, there is no need for tables and chairs. However, it is also common to provide $\mathrm{CL}$ services in other forms without using a building or having a permanent location. In fact, CL2 developed from a WA project that brought books in a large plastic box for children in the urban poor community to read. Their Book House project then equipped the volunteer's house with a bookshelf and later the city municipality provided funding to build a prefabricated mini library, the current CL2. As an illustration of how flexible arrangements can be, other WA projects at community level used mobile libraries in vans and motorcycles with book carriages on the sides.

In terms of the physical condition, the WA stressed that the building needs to be inviting and attractive. However, it must not be luxurious or very formal or look like 'a holy place' that might intimidate disadvantaged people. The officer in CL2 wished for funding to renovate the $\mathrm{CL}$ for the users to see that the library is new so they will want to use it more often.

Both sites were located in the place where there was a great number of young children; CL1 was next to a childcare centre while CL2 was the only positive space available in the urban poor community. Therefore, $\mathrm{CL} 2$ also functions as an informal childcare centre. 
My friends ask if I am a library officer or a nanny...Our community is the most disadvantaged urban poor community in this city. The disadvantaged children have nowhere to go. They have to come to the library. Their parents also tell them that "go, go to stay at the library, it is open (CL2 Orada).

The observation and interview confirmed the severely deprived environment of the urban poor community. The officer told stories about her daily routine in which she sometimes needs to deal with drunks, people who are addicted to narcotics, and drug dealers. The dealers often want to leave their children with her and make their deals in a house next to the library. These circumstances, while difficult, nevertheless made the municipality realise that there is a need for a $\mathrm{CL}$ for children in this at-risk area. The municipal officers talked about the childcare situation and problems at the location that confirmed the issues that the CL officer faced. The situation in the 'at risk' area therefore affected the $\mathrm{CL}^{\prime}$ s operation and $\mathrm{CL}$ officers. It also redefined the $\mathrm{CL}^{\prime}$ 's functions.

\subsubsection{Key Individuals and Training}

Evidence presented above showed that leaders in RfP in Case 1 played significant roles in initiating, fundraising, establishing the library, and training library officers in reading to young children. Documentary evidence showed that, as the concept of RfP is new in Thailand, the Facebook pages of the prominent leaders in RfP in Case 1 were often used to share simplified knowledge from other leading RfP individuals, for example, Jim Trelease.

Participants in both CLs agreed that parents are the most important role models in encouraging children to read for pleasure. Parents or close family members who take care of children need to read books themselves so children can see the pleasure, the intrinsic reward they can get from reading. A participant in CL2 who is a middle class father of 5 and 11 year-old sons mentioned the different impact of being a model who 'acts' and being a commander who 'tells': "I read the huge translated Chinese work, the Epic of the Three Kingdoms and my eldest son who is 11 years old also reads it. But, if I tell him to read, he will not read it" (CL2 Chaiya).

Overall, the findings suggest that there are several desirable characteristics for reading promoters. First, the promoter needs to be a local, friendly person, familiar with the 
community environment that can help attract library users. Second, they need to have a volunteer spirit and be pragmatic so they can go "the extra mile." This is because in deprived areas, circumstances can be extreme, as in the urban poor area mentioned in 6.5.4. Last and most important is a knowledge of books and being readers themselves. The WA expert asserted that these were the rarest characteristics generally found in librarians in Thailand. He also mentioned that these key characteristics were more crucial to the success of reading promotion than the number of books.

In terms of training for staff, CL1 and CL2 revealed the different training opportunities and job security prevailing for CL officers. In CL1 the temple initially had no budget to hire a permanent officer so the $\mathrm{CL}$ relied on people from the WA, and nursery caregivers who were trained by the WA in 2008. However, when Karuna was hired in 2009 she had no opportunity to be trained. She learned how to use picture books effectively during storytimes when the WA sent some officers to help take the books out to the community for another project. She talked about training, knowledge, and confidence:

Knowledge and capability building are very important for library workers. If I have knowledge and experience, I can properly assist the children who come to use the library. I'm using my own thinking and my experiences. I do not know whether it is right or wrong because I have never been trained. I'm not confident... At first, I still didn't know what to do. I saw the kids turning the pages of the picture book. But I thought that they looked like they wanted to know the story. So I decided to read to them and let them see the pictures. From then on, I started to read to them every day (CL1 Karuna).

In $\mathrm{CL} 2$, however, Orada, the $\mathrm{CL}$ officer, was the only one who had a variety of training. She was the only one left of the first generation officers since the establishment of the $\mathrm{CL}$ project so she was highly trusted and was selected to be trained so that she could train other officers. She wanted all officers to be directly trained by the WA because when other $\mathrm{CL}$ officers are trained by her there are questions in terms of reliability. However, training by the WA is not possible because, as the $\mathrm{CL}$ coordinator explained, $\mathrm{CL}$ officers are not in the municipal system; they were hired on contracts so the municipality cannot reimburse them for training and other expenses. The $\mathrm{CL}$ officers also faced a lack of job security. Orada explained that with pay of only 6,000 baht (240 NZD) per month (200 baht or 8 NZD per day) it was difficult to find officers to work for the CLs. 


\subsection{Chapter Summary}

Key individuals in Case 1 provided both convergent and contradictory views on the four major factors identified. Case 1 contributed to both the understanding of RfP in Thailand and the operation of CLs with full support from the local authorities. The case reveals point of view and knowledge of both leaders in RfP and local reading promoters, who are from the same type of library but from different community contexts. While leaders in RfP understood and were able to comment on the national reading policy and situation, local $\mathrm{CL}$ officers gave their local views, reflecting important understanding from both service providers' and users' perspectives.

The case demonstrates that book access is a major theme touching almost every factor. As a majority of the stock in both CLs were picture books, literacy development was also mentioned throughout all factors, ranging from the MOE policy, the literacy tradition, the book market, and the training of family members. Class divisions preoccupied rural and people in urban poor area people and prevented them from exercising their right to access reading materials from public libraries, underlining the significance of CLs.

The traditional image of the strict, formal or 'holy' library and unfriendly librarian was mentioned. Case 1 shows that when leaders in RfP can build a contrasting and desirable modern low anxiety atmosphere, CLs excel in attracting more people to the libraries. However, the prominent leaders in RfP contended that in the prevailing reading promotion situation, the need for investment to create new readers is more important for Thailand than investing in building new libraries. 


\section{CHAPTER 7 Case Report 2 \\ CLs under ONIE}

\subsection{Introduction}

This chapter outlines case findings from CL3 and CL4, successful Smart Book Houses (SBHs) under ONIE. They were greatly affected by budget cuts for newspaper and magazine subscriptions in 2015 , as mentioned in section 2.5.3. In both CLs, there are a mixture of people involved in the running of the library who work variously in the agricultural sector, service sectors in the city, and government officials.

CL3 is in a fertile agricultural village in a small district close to a major city. The village head has volunteered to give a space in front of her shop to be the CL since 2014. The village head, Hatai provided comments from both community leader and reading volunteer perspectives. CL3 is the only case in the study involving Lak, a district public librarian (DPL), the leader in RfP in this $\mathrm{CL}$. She is responsible for a district public library in the township (district) area but also voluntarily works with active sub-district ONIE teachers (SOTs). Another participant is Thana, the most effective SOT who have volunteered in ONIE's village reading room for more than ten years.

Similarly, CL4 is also located close to a major city but in another part of Isan. The village head contributed a piece of land to build a community centre. The CL shares a space next to a community shop within the centre, hence the shopkeeper also plays a part in taking care of the CL. The crucial factor making CL4 very successful is a former ONIE executive, Tassanee, the leader in RfP in this case, who had acquired large collections from several agencies. The other two participants, an active SOT (Kitiya) and an active ONIE reading volunteer (Jira) provided understanding of RfP practice. 


\subsection{The Thai Reading Context}

\subsubsection{Literacy Problems}

Participants in Case 2 stated that literacy problems are the most fundamental factor affecting RfP. Since they specifically commented on 1) MOE's literacy policy (7.2.3.1), and 2) reading to young children (7.3.1.3) those points are addressed separately in their respective sections.

\subsubsection{National Reading Policy}

The national reading agenda, Decade of Reading 2009-2018, affected Case 2 the most in the study because CL3 and CL4 were amongst the CLs established as a part of the agenda. The SBH Project is the largest CL project in the country, initiated by ONIE in 2013 in 41,800 villages. CL3 and CL4 greatly benefited from the project because daily newspapers, a weekly review magazine, and a monthly magazine were provided to each CL. Participants confirmed that although the villagers were not specifically aware of the agenda, they greatly enjoyed the publications.

A leader in RfP talked about reading promotion in rural areas, and the effect on CLs after the serial publications cut in 2016:

When there are no serial publications in CLs, reading promotion in rural areas is a struggle. The government launched a policy on reading that focuses only on the urban population. As rural people have no reading materials, they simply have nothing to read... (CL 4 Tassanee).

She also commented on policy instability due to ONIE executives' and politicians' subjective views on reading, leading to their disagreements and changes in reading policy.

An active SOT in CL4 and a village head in CL3, who were much affected by the serials cuts questioned the point of the agenda. According to them, the general quality of reading materials provided to ONIE CLs is poor. Moreover, while there had been serials in SBHs that had significantly ignited people's reading interests, subscriptions were gradually cut after only two years. 
Concerning awareness of RfP, most participants agreed that reading promoters were responsible for making people be aware of RfP. They believed that if villagers understand great benefits of reading to young children, they will have more awareness of RBP and will support it

\subsubsection{MOE Policy}

\subsubsection{MOE Literacy Policy}

The most active SOT (Thana) suggested that the success of this policy depends on ONIE reading volunteers because they are in the community and know the children and where they live. The DPL confirmed Thana's view that he worked well because he had a volunteer team to support him. In addition, Thana commented that the early literacy programme takes time, but it is valuable. It will be evaluated after 5 to 10 years.

As the district ONIE is under central ONIE, it needs to accept this MOE policy. There was a list of reading activities that included a mixture of reading attainment and creativity stimulation. There was also a policy on cooperation between the library and library members, and a plan to improve the physical environment for the young users and provide an officer to take care of them. However, the central ONIE had not provided funding for the acquisition of reading materials and the improvements mentioned. Another document, a daily/weekly plan for the 2016 ONIE literacy policy shows the negative impact of the new policy on ONIE teachers' workload.

\subsubsection{OBEC School Libraries}

Most participants viewed that school libraries in Thailand have not functioned well because there was no librarian position in the school libraries and the books acquired were not appropriate to encourage children to read. The DPL shared her experience in school libraries as a person who was invited to assist with organising the library, indicating that she could not organise the collection because it was minimal and only textbooks were provided. There were no books for RfP. Moreover, teacher librarians always have a heavy library workload added to their teaching routine, and they normally do not want to do it. However, one exceptional case highlighted by Tassanee showed that a rural school library equipped with quality picture books and informed teachers 
could have a large impact on the literacy development of children in poverty, as Krashen (2014) asserted.

While ONIE-supported CL3 had no picture books, CL4 on the other hand had a great variety of materials for children to read for pleasure, acquired through the support of several organisations. A SOT asserted that primary school students liked to visit CL4 because there were more books which suit their interests, for example, comics. The village head in CL3 also confirmed that she could not see how the school encourages students to read for pleasure because the school library did not have suitable reading materials. This evidence suggests that the school library inadequately supports RfP and thus highlights the crucial role that a $\mathrm{CL}$, equipped with a great variety of suitable materials, can play in supporting RfP for children in rural areas.

\subsubsection{ONIE Public Libraries}

Participants agreed that country people did not visit the district public library, which had relocated from a temple to the district offices. Thus, only people who live in the township area who can access the library within walking distance use the public library. They were mostly the civil servant officers working at the district offices and their children, and souvenir sellers. Participants found that when there are interesting books brought from the public library to the village, the villagers are very eager to borrow those books. However, they do not visit the public library for two reasons; they need to travel by motorcycle or car, and they feel uncomfortable wearing their dirty working clothes in the formal public library.

The leader in RfP in CL4 suggested that public libraries in Thailand need to change the way they operate: "I think it would be better if they acted proactively than just traditionally... we need to distribute the books that we have in public libraries to their communities" (CL4 Tassanee). The suggestion is, in fact, just what the DPL in CL 3 regularly does and she has received numerous awards for her outstanding efforts in linking the public library with CLs. However, these kinds of activities are outside the DPL job description. The DPL commented that because she proactively works more than her allocated duties, many SOTs did not like her and did not cooperate with her (details in 7.6.1). 
Since district public libraries in Thailand are annually allocated only about 30,000 baht (1,250 NZD) for book acquisition, each district library can buy only a limited number of new titles annually. The DPL talked about budget limitations: “...this amount of money cannot buy many books if a book costs $400-500$ baht (16-20 NZD). I choose the books which are small and colourful to attract readers in rural areas. I also buy children's books, but they are quite expensive" (CL3 Lak).

In her view, the best way to promote RfP in CLs is the provision of reading materials that go through the public library and then are distributed to the CLs. However, these CLs are not officially within the public library system. As a DPL, Lak's effort with the CLs in the district were her own initiative, agreed by her director. She talked about her work with the public library and with CLs:

\footnotetext{
For me, I am busy and not feeling lonely [in the public library] because since I worked here, I have promoted reading corners in the community for 56 villages. It's nearly all villages in this district. When I go to work out of the public library, there is another officer who will take care of the library. He doesn't have a degree in librarianship though. I seldom work at the library. Thana, an ONIE teacher, is my buddy on community reading promotion. (CL3 Lak)
}

Although her work with several SOTs was outstanding and the ONIE director was pleased with her work, her conditions of employment are lower (as a non-government official) than the ONIE teachers who are fully employed as government officials. Several SOTs taunted her because she worked beyond the scope of her position. However, if she did not do that, $\mathrm{CL} 3$ and other SBHs in the district would not have quality reading materials from the district public library. Since community reading promotion was not her direct responsibility, the development of RfP in CL3 was fragile. She mentioned she became less active after Thana was moved to work in another CL. Similarly, a SOT in CL4 said that without the retired ONIE executive, who was 70, CL4 might not get more books and will therefore be unsustainable in the long run.

The DPL, who was respectfully called "Teacher" similar to the SOTs, was much affected by the early literacy policy as it required her to report the literacy rates in ONIE CLs in the district shortly after policy implementation. She talked about the impractical policy, and coordination with SOTs. She showed that there is an ambiguity in the reporting lines 
in district public libraries and $\mathrm{CL}$ initiatives because SOTs are not legally required to report to the DPL, and CLs are not under the authority of the district library. However, practically, the DPL needs to work with these teachers on CL projects in the district. As a result, the DPL had no authority to ask them to provide a report on this MOE policy to her but she needs to estimate the figures to satisfy the district ONIE director.

\subsubsection{ONIE Smart Book House (SBH)}

As noted above, rural people did not like visiting the public library and preferred to visit the newspaper reading corners that were first established around 30 years ago then transformed into village reading rooms (see section 2.5.3). After adding more serial publications in 2014, these easy access volunteer-led reading spaces were changed into SBHs. The active SOT viewed SBHs as beneficial for all community members and a former ONIE executive who was a leader in RfP in CL4 asserted the importance of this form of $\mathrm{CL}:$

The reading culture in Thailand has not progressed well. Therefore we need to take books to them, rather than waiting for them to find books, especially in public libraries [which they do not visit]... I disagree with the idea that the reading culture is not a Thai tradition and cannot be nurtured here. I think rural people don't read due to several factors, for example, no books to read, illiteracy, no time to read. We shouldn't look down on them by concluding that they won't read if they have books provided (CL 4 Tassanee).

A SBH Project document in 2015 provided figures stating that the project expected to use 430 million baht (17.2 million NZD) to serve 2 million economic and linguistic disadvantaged people in a total of 77,280 villages in the country.

The DPL who brings books from the public library to villages commented that the government should provide more funds to acquire reading material for SBHs because during her 11 years' experience she found that rural people are eager to read as long as they can access books that suit their interests. The villagers' eagerness to borrow books, provided they are of sufficient quality and variety, is considered in 7.5.1.2 and the effect of budget cuts on book acquisition is discussed in 7.5.1. 


\subsubsection{Coordination Between Supporting Organisations}

Participants strongly agreed that coordination between organisations and individuals can be powerful and positively impacts CLs. This was demonstrated clearly in CL4 which received support from numerous organisations. However, a leader in CL4 identified the importance of having one organisation that plays a leading role in the network. In her experience, cooperation depends on the community's strength and the ability of organisations to work as a network.

Local reading promoters also stressed the importance of cooperation, suggesting that a community reading project needs, at the very least, support from the community, the head of the village, or the TAO, companies, or stores. The SOT in CL3 wished that both public and private sectors would collaborate in running activities for the national benefit. As he observed, mostly the public sector coordinates the activities, even though the private sector has greater financial means to support RfP.

An active SOT in CL4 also commented that the transfer of literacy projects from ONIE to TAOs is not practical because TAOs have much less experience in coordinating, distributing, and promoting the use of reading materials. The lack of expertise resulted in piles of undelivered daily newspapers in TAO offices. The SOT showed her disappointment for the waste of the budget. She also shared her strategy for newspaper delivery. She is responsible for 15 villages but she cannot reach them all. Thus, she often asks for help from the village heads and the delivery goes well in her area.

CLs cannot avoid cooperating with TAOs in relation to RfP, but the former ONIE executive commented that TAOs have two major problems. First, their weakness is in education support for the villagers because they have no expertise on educational matters. Second, their offices are generally difficult for villagers to access because TAOs have been established only in the past ten years and they often cannot find a location at the heart of the community. Indeed, unlike SBHs, TAO library corners are not in the centre of the village. 


\subsubsection{Book Market}

Some participants said that the high cost of books prevents people from owning books and may be one of the reasons people do not read extensively. However, a book fair initiated by Tassanee that brings publishers from Bangkok to meet readers in the region is proof that Isan people have sufficient purchasing power. She began the fair out of a desire to give people the opportunity to access books titles that they really want to read. The event was also a chance for CL4 to select new books, the purchase of which was philanthropically supported by the department store organising the event.

In Case 2, participants agreed that people's purchasing power was not similar to that in Case 1 because both CLs in Case 2 were in fertile agricultural areas in which people have a variety of career choices and more disposal income. The village head said, for example: "If parents are interested in a book, they buy it. Rural people can afford books if they are really interested in it." Tassanee also stressed the connection between the opportunity to access books and RfP:

I insist that reading in Thailand is not about class. It is because middle-class people have greater access to reading materials. Rural people sometimes can access only boring donated books. When we subscribed [and distributed] more relevant magazines, they did read more. Thus, to give people the chance to access reading materials is crucial" (CL4 Tassanee).

Her assertion conformed with Krashen's assertion that people will read if libraries supply books that meet their reading demands.

\subsection{Thai Cultural and Social Factors}

\subsubsection{Literacy Traditions}

\subsubsection{Teacher-based Learning Style and Home-School Partnerships}

Participants' comments on the Thai literacy tradition reflect a teacher-based learning style that is still prevalent in rural areas. Tassanee viewed it as the predominant norm that prohibits RfP: 
The misunderstanding of child literacy development has caused most problems related to RfP in Thailand. There are the parents' norms as follows. First, literacy development is solely a school duty (CL 4 Tassanee). [This quote is continued later.]

She asserted that most villagers view literacy development as absolutely a teacher's duty, therefore, parents tend not to involve themselves with reading to young children. Participants also stated that many children lack the opportunity to develop to their full potential because of the absence of home-school partnerships. They said it was most severe among children who were with their grandparents, who cannot provide proper literacy support. Hence, the school tends to be solely responsible for the literacy development of young children in rural areas.

Participants in both CLs agreed that nowadays, after seeing the positive outcome of BSPs, most parents started to understand that they need to engage in literacy development and are gradually changing their attitudes about home-school partnerships.

\subsubsection{Knowledge-Oriented Mind-Set}

Most participants agreed that villagers and early childhood teachers in the villages often hold a strong knowledge-oriented mind-set so they normally buy Thai $A B C$ books for their young children and have the view that books should contain knowledge. The village head in CL3 talked about a common Thai reading scenario in which the parents' generation does not understand the attractiveness of light reading and the benefit of RfP for children:

If adults see that children do not read a textbook, they always reprimand the children for reading for pleasure, rather than a textbook. [I saw that] children read what they want to read, but parents do not understand the children. Most parents would buy books providing knowledge rather than books for RfP, for example the alphabet book for small children (CL3 Hatai).

She suggested that these demands from authority figures might result in the avoidance of children to read and learn in general, especially teenage males. She also added that "Some older children [age 11-12] are resistant to this teachers' or parents' reading mindset because they want to play or read for pleasure. Focusing only on studying makes them stressed" (CL3 Hatai). 
The DPL noticed the same mind-set in ECTs who were hired by TAO. She commented that these ECD teachers had only finished high school (grade 12) and had not been trained so their approach was that children should be able to memorize and write the Thai alphabet. If children cannot write the alphabet, they often focus on this "inability," and tell the grandparents or parents. She thought this was inappropriate child development practice. "These children are only two or three years. I think it is not right because I have been trained [by WA] that children at this age should sit on a lap and listen to storytelling and look at beautiful pictures in picture books" (CL3 Lak).

The leader in CL4 described four parental normative practices that she considered the primary causes of low literacy development and RfP problems in Thailand. The first norm regards schools as solely responsible for literacy development (see7.3.1.1), and the other three are as follows:

Second, literacy development starts by memorizing the 44 Thai letters of the alphabet. Parents normally buy an alphabet book to teach their children and that makes them feel stressed. Third, rural parents don't know about RfP; they haven't seen such activity before. When they were at school, there were no books to read for pleasure. Nowadays, there are still no such books in rural areas. Here there are only alphabet books. Fourth, parents tend to force their children to read and write when they aren't ready. They expect that the CDCs will teach the kids to write. The expectation makes kids feel stressed, hate books, and hate school. There still are a lot of parents that think that Thai alphabet writing is more important than reading encouragement (CL 4 Tassanee).

In addition, participants mentioned a normative practice in which parents compare their children with other children and worry that their children will not be as academically able as their peers. Such obsession hinders RfP from a young age, Tassanee, for example, suggested that it prevents children from having appropriate literacy development and the opportunity to enjoy reading and develop positive attitudes toward reading.

\subsubsection{Reading to Young Children}

Reading to young children was an innovation the leaders in RfP brought to both CLs. The Bookstart Project (BSP) can influence parents' and grandparents' attitudes if they can see changes in children. However, initially, it was the responsibility of leaders in RfP and reading promoters to introduce the idea of reading to children and find practical ways 
to do it. Leaders in RfP strongly asserted that villagers who were trained in the concept welcomed this new idea.

This fundamental concept, however, needs to work against the four norms on literacy development held by parents mentioned in the previous section. She noted that purchasing power can be a problem as books are still expensive for the villagers. However, she estimated that one out of four families in rural areas that joined a BSP and see the importance of picture books would start to buy picture books. Thus, she thought a book gifting programme was still crucial for people in rural areas.

In CL4, the initial success of a BSP which started in 2010 with packages of three picture books given to 20 families, was later extended to become a large library collection supported by an international charity. This library model did not include the building but was a collection of 238 selected picture books, children's books, and books for parents. In addition, Happy Reading later gifted books to the $\mathrm{CL}$, making it one of the outstanding CLs in the area with more than 500 new books.

In CL3, however, picture books were not provided, and they needed to rely on picture books acquired with the public library's limited annual budget. Moreover, there were more grandparents that SOTs needed to work with. An active SOT talked about the persistent effort they need to make to instil the practice of regularly reading to young children:

\footnotetext{
At first when we enter the community, grandparents read to young children with us. However, when we walk out of the community, there are normally only ten people out of 100 doing it continuously. We needed to go into the community many times. Telling them to do it only one time does not work (CL3 Thana).
}

Overall, only direct experiences convinced people of the benefit of this new reading practice. Even the DPL, who is a reader and initially knew about reading to young children, was not convinced of its value until she saw the tangible positive outcomes for her niece who read to her young daughter.

Another statement showed that though they lacked educational opportunities, a small number of villagers are progressive. The leader in CL4 talked about a mother who started to read to her child before the BSP team entered the village. Although she needs to 
adjust her book selection to suit the child's age, she is a reading model for other families in that area. This observation confirmed Tassanee's assertion about class and RfP (section 7.2.5).

\subsubsection{Learning Atmosphere}

\subsubsection{Low-Anxiety Atmosphere}

A male ONIE teacher talked of the high-anxiety atmosphere in his school that resulted in school dropouts, so he was aware that the majority of his ONIE students need a subtle, low-anxiety learning and reading atmosphere. He was critical of the prevailing Thai authoritarian tradition.

Most participants agreed that CLs which are physically easy to access and have a relaxing atmosphere encourage library visits. A leader in RfP stressed that ONIE needs to bring books close to the villagers because they don't like to go to the formal public library for the reasons of discomfort mentioned in 7.2.3.3. The village head in CL3 who offers her house as a CL talked about the relaxed oral tradition and her friendly attitude that invited villagers to use $\mathrm{CL3}$, which had helped to create community dialogue.

\footnotetext{
I always open my house. People will come and have a conversation here. When it was a shop, it was also a village meeting point. I know a lot of people, so they drop by and read. It is very different because a newspaper is a great motivator to read... When they are free, they come to read here, and I also make them coffee. People also have a chance to talk to each other... If there are no newspapers and magazines, I will be lonely because no one will come to my home. I like to talk to others (CL3 Hatai).
}

Since Thai traditionally do not use chairs and tables but sit on the floor or use low daybeds to sit and write, the seating arrangements in the village head's home generates a low-anxiety atmosphere for villagers. In CL4, an ONIE reading volunteer explained that similar physical factors contribute to a comfortable feeling for the villagers, which makes it different from the public library in the city. Children can take books to read under the trees if they prefer.

The interview with the DPL partially reflected a traditional belief that the public library does not welcome people to spend time just relaxing in its space, particularly truanting students who the DPL worry will be violent and vandalise the library. 


\subsubsection{Sharing Reading}

All participants strongly agreed that CLs must be places that people feel comfortable to be together and enjoy discussing local news. The oral tradition and collective social structure makes people feel good when gathered together. The leader in RfP talked of the social dimension of reading: “...in our collective society each reader doesn't just read, they will tell other family members or neighbours about what they read. The information and knowledge will be distributed in the form of socialisation" (CL 4 Tassanee).

The village head in CL3 talked of the pleasure villagers derive from sharing their reading of the serials provided through the SBH project:

\footnotetext{
We also have Koosang Koosom Magazine. Some people read about agricultural technology in Technology Chao-ban Magazine and then they discuss it. This is good. They can also read and tell and exchange stories with others. This can be pleasurable (CL 3 Hatai).
}

Similarly, in CL4 the reading volunteer added that parents are happy to have friends read together to their young children at the $\mathrm{CL}$. She also suggested that both peer cooperation and peer pressure supports RfP: "Reading alone and group reading are different. If we read alone, we will feel lazy after a while; we will be more alert when reading together. Children will feel like it's a competition, some read aloud to each other" (CL 4 Jira).

The leader and the SOT in CL4 also talked about the pride or "face" that children and parents gained from shared reading activities, for example after school, children will read together at the $\mathrm{CL}$ and the older children would teach the younger ones. The activities contribute to children's and parents' pride, and peer influence. The reading volunteer also often mentioned the villagers' pride when guests or professional visitors are visiting CL4 because the $\mathrm{CL}$ is evidence of their collective efforts.

\subsubsection{Normative Culture and Feminine Society}

All participants agreed that villagers who live close to the city are not normative and are becoming more open to reading to young children and RfP. They mentioned that reading promoters and SOTs need to know how to communicate and do it continuously to promote a better understanding of the benefits of RfP. A reading volunteer mentioned 
the need for local leaders who can regularly stimulate villagers in reading activities, which is evidently true in CL3 and CL4 that had active and dedicated community leaders. A subtle aspect of the feminine society is that most people try to be in the mainstream; evidence of this is when a reading volunteer talked about how parents compare themselves with their peers and decide to buy a smartphone for their children. She mentioned Saranya, a SOT who was determined to avoid the mainstream by not purchasing smartphones for her children, even if the price came down.

...Some people would accuse her of being too thrifty as a smartphone now costs only 500-600 baht (20-24 NZD). Why don't you buy it for your kid? They think other people can afford it, why don't I? (CL4 Jira).

Jira's worries about smartphone addiction and its impact on RfP are further presented in section 7.3.5 Media.

\subsubsection{Attitudes towards RfP}

All participants had a positive attitude toward RfP although they considered most villagers to have a limited awareness of the value of the practice. Case 2 also showed that in a collective society RfP, explicitly reading picture books to young children, needs to start as a collective social activity (see section 7.3.2.2.)

The case also showed that in the rural Isan context RfP might be connected with both leisure and part-time job opportunities, as presented in 7.5.1.1, or could even indirectly affect a career, as reflected in these comments by a former prominent ONIE executive.

In rural areas, people read for pleasure, and some read to fulfil their needs. It depends on the age group. Young and older children read for pleasure. Many adults read novels and series synopses [from daily newspapers]. Working people read about career opportunities, and some of the elderly people read for pleasure, such as books on how to make use of local plants. I think in the Thai context, whatever we choose to read brings both pleasure and knowledge at the same time (CL4 Tassanee).

This explanation reflects a compromise or combination of the Thai strong knowledgeoriented mind-set and the new RfP concept introduced from the West. 


\subsubsection{Media}

All participants expressed their great concern about the excessive use of technology in Thai society, especially among young children and teenagers. The DPL in CL3 talked about the elderly and parents in rural areas having limited understanding of the impact of screen technology on young children. The PDL saw that during her 11 years of working, modern media had led to a deterioration of RfP in her public library. Most importantly, a SOT in CL4 also estimated that around 90\% of caregivers in 15 villages under her responsibility seemed to raise children via android and tablet PC. She said that when these children see picture books with no movable pictures they are not able to concentrate on these physical books. Similarly, the leader confirmed that the smartphone culture greatly affected reading promotion nowadays.

\footnotetext{
...due to the smartphone culture nowadays, reading promotion will be greatly affected. People use it both in their free and not-free times. When I been to all areas in Isan, I have seen people addicted to smart phones, both children and adults. It's hard to control. The problem is that it we waste a lot time with it and don't want to do other activities. At present, the major problem affecting RfP is the loose control of smartphone use in families. It's true that villagers don't know the side effects of smartphone addiction in young children (CL4 Tassanee).
}

In CL 4 all participants strongly agreed that villagers do not know enough about the side effects of smartphone addiction for literacy development. The reading volunteer in CL4 had a lot to say about the free smartphones and affordable internet plans that lure rural people. Her experience was that her cousins' children and CL users spent less time on free play and $\mathrm{CL}$ visits once they had smartphones.

Another medium that participants commonly saw children use in rural areas was 'story $\mathrm{CDs}^{\prime}$ in village childcare centres. They found that reading to young children was rare among early childhood teachers who usually use CDs with children.

Most participants thought that reading promoters need to think creatively about new ways to promote digital reading via social media or smartphones. 


\subsubsection{Religion}

There are two religious aspects discovered in Case 2: volunteer works as merit-making; and pleasure from the ability to do spiritual crafts learned from books provided by CLs. Several participants who live in rural areas said that they devoted themselves to charity works because they believe it is merit making, a core element in Buddhist belief. The DPL used her volunteer mother as a role model; therefore, she devoted herself to distribute books to others in rural areas.

Villagers in both CLs were pleased that the DPL, CLs, and SOTs provide access to handicraft books so they can make religious handicrafts (see section 7.5.1.1). This reflection was confirmed by archival photos and interviews with SOTs who gladly responded to this specific need of the villagers.

\subsection{Isan Socioeconomic Influences}

\subsubsection{Education, Poverty, and Migration}

An active SOT who finished high school at 18 and went to Bangkok, as do many Isan, stated that he started to study for a degree at 40 . He portrayed the life of a common Isan aged 50, saying that a large number of his friends who had lower levels of education work for a 300 baht (12 NZD) daily wage. He commented that with that difficult life, villagers could neither afford books which cost 100-200 baht (4-8 NZD) nor have time for appropriate parenting.

Participants agreed that a lot of Isan people leave their children with grandparents. Therefore, nurseries and schools have to be solely responsible for the literacy development of these children. The DPL commented on the parents' migration and its impact on literacy development.

Grandparents leave literacy development to the school. If the caregivers in the nursery have not been trained, they will only traditionally lead children to recite multiplication tables and the Thai alphabet. Grandparents tell their grandchildren to learn and gain knowledge from teachers because they are tired and old... So, they leave grandchildren with smartphones and tell them to search for information on the internet (CL3 Lak). 
She said that one of the most problematic aspects of parenting by the elderly in rural areas was that they tell their grandchildren not to go to the CL because they think their grandchildren only play there. She mentioned that very few people support their grandchildren going to the library because they are also afraid that their grandchildren will have an accident.

The DPL commented that the grandparents usually have insufficient modern parenting skills and some have eyesight problems. They normally tell the children to eat quickly, watch TV and then go to bed. The most significant problem affecting RfP in this context is that most grandparents still have conservative ideas on books and reading. They do not have books in the home to read to the children and they think there is no need to read because young children will not understand. They felt that this is negatively affecting RfP in rural Isan areas. A reading volunteer asserted that even when those grandparents were provided with three picture books in the BSP package, she did not think they touched the books (CL4 Jira).

\subsubsection{Isan identity and Isan Language}

As mentioned in relation to the typical Isan profiles of participants in the previous section, participants agreed that Isan identity has an impact on Isan children and adults who speak the Isan language especially in the city and civic spaces, for example public libraries. This affected their self-confidence and deterred them from approaching people and asserting their rights to access books. The DPL mentioned that Isan people are generally shy and humble so they are afraid to do many things in the city.

Case 2 showed that using Isan as a mother-tongue language affected CT proficiency. Most participants agreed that delayed CT acquisition resulted in delayed RfP. They mentioned that teachers need to use both Isan and CT languages with children at the entry level (grade 1-3) and Isan children need several years to adjust to CT and reading materials. With no children's books available at home, school is the only place they are exposed to CT reading materials.

Most participants also agreed that parents using the Thai language with their children from a very young age yields positive outcomes for RfP. The children will be more confident, more capable of using $\mathrm{CT}$, and can read fluently. 


\subsection{Community Library Factors}

\subsubsection{Reading Materials}

It was mentioned in 7.3.3.4 that book access is the key for RfP in CLs, and all participants confirmed that library materials are the most crucial CL factor. However, a SOT and the DPL asserted that book access needs to be coupled with activities and reading promotions to make rural people engage with library materials. Specific characteristics of reading materials affecting RfP in the CLs presented in this section are 1) responsiveness to local needs, 2) variety and quality, and 3) picture books and light reading.

\subsubsection{Responsiveness to Local Needs}

The leader in RfP in case 2, the oldest CL initiative, who had worked with ONIE for more than 30 years, talked about the development of rural reading promotion in Thailand which started 30 years ago. She also talked about the popularity of newspapers, and the condition of reading materials in ONIE CLs, which highlighted the importance of reading materials that suit local needs.

When I look back...the village reading room was an excellent investment. Newspapers are so cheap, and if only five people read it, we achieved our goal. Villagers love to discuss current situations. Actually, the government invests very little in the CL overall. There is zero investment in space/buildings. These reading rooms and book houses are simply volunteer places that are appropriate for villagers to access. When there are newspapers and books available in rural areas, I see that some country people do read. Probably, people who don't read cannot find the books that suit their interests. Most of the books commonly found in these ONIE CLs are unattractive donated books which are limited in number (CL4 Tassanee).

All participants asserted that the budget cut for daily newspapers significantly affected RfP because villagers at both CLs used to like sharing what they read, and discuss news in the late morning or evening after their farm work. In both CLs there were around ten people who came regularly on a daily basis to the CL for the newspapers. The DPL noted that it was very different when there was a budget for magazines and newspapers. Since she was the person who subscribed to those serials, distributed them to CLs, followed 
their usage, and adjusted them to local needs, she was regularly asked why the serials had disappeared from the library. She said Thai newspapers provide issues for discussion, and include serialised novels and lottery results, all suited to RfP.

She further talked about the situation that more than one person asked for the newspapers at the same time so she needed to bring copies of old newspapers from the public library. It portrayed the reading need of villagers, supporting the assertion of the former ONIE executive and Krashen, that if there are interesting reading materials provided, villagers do read. Similarly, in CL4 a reading volunteer gave a lively picture of newspaper use in the rural areas.

\footnotetext{
The newspaper is very popular; it did make a difference. As the library operates within the community shop, when people come to buy goods here, they will read if they see a current daily newspaper... People love to stop by to read if we have a current newspaper next to the shop. They normally discuss the news... Weekly magazines and women's magazines are popular among women. They will borrow Koo Sang Koo Som and Kwan Reun to read for general knowledge and crafts (CL4 Jira).
}

The case showed that villagers preferred to read books and magazines that have handicrafts sections because female villagers can make ceremonial crafts and sometimes generate more income from crafting.

\footnotetext{
I think this $\mathrm{CL}$ is very useful... We are happy that now we can do a beautiful fresh banana leaf and flower offering to offer to the Buddha on Buddhism Lent Days...Another craft that we learned from books is a variety of dana coin decoration which will be used in large numbers when we do merit in important Buddhism ceremonies (CL4 Jira).
}

This is an adjusted RfP definition relevant for this rural area that includes both leisure and part-time job opportunities as mentioned in section 7.3.4.

Active SOTs in both CLs confirmed that the adverse effect of the budget cut was that: 1 ) there were fewer users visiting the CLs 2) people feel frustrated about the lack of availability of serials, 3) SOTs did not have a tool to start a dialogue with villagers and ONIE students. They worried that without additional reading material, any RfP practice established will stop and the CLs will not be sustained. 


\subsubsection{Variety and Quality}

Participants agreed that the lack of variety and quality of reading materials are greatly affecting RfP in CLs. They confirmed that normally reading materials in CLs are not attractive, as mentioned in the previous section by the leader in RfP. The DPL talked about the quality of reading materials selected by ONIE and her book screening.

I think many of the books that ONIE has provided for the community to support RfP and create the reading habit in rural areas do not serve the purpose. The ONIE directors did not know how to select quality and practical books [for villagers]. They selected books which were poor quality, and people did not read them... I select books [from those supplied by ONIE] before distributing them to the community because I do not want them to become junk... I have learned from experience... When I go to the community, I am the first who is blamed by the villagers that they have been provided with those old and poor quality books" (CL3 Lak).

The DPL made additional comments about the quality of the books and eagerness of villagers to borrow them.

...They are hardcover books, for example, books about handicraft, cookbooks, and Dharma books. The villagers like these kinds of books and they have never seen such glossy books. I let them borrow six books and told them to return to the SOTs... I let them borrow only six books because those books are expensive [cost 400-500 baht (1620 NZD) per item].

It is one way of encouraging people to apply for a district library membership... In reality, they cannot access us if we do not approach them... I just hope that they read and inspire their children. Yesterday I sympathised with them because they said that they want to read books, but the public library is very far..." (CL3 Lak).

She also commented that the book donation project, the Book Voyage project, which was established after the subscription cut, would not work because the books that the villagers gave her were very old and torn cheap novels and joke books. Those books generate more work for delivery and weeding.

It was different in CL4 which was supported by several organisations. The observation showed that there was a great variety of books for young children, teenagers, and parents including high quality donated magazines. Books were in excellent condition, 
and borrowing records showed a great variety of good quality books selected by WA (similar to CL1). Picture books and translated Korean non-fiction graphic texts were very popular. Teen novels had also been frequently borrowed. However, there had been no additional titles added since its establishment, so library visits had declined.

\subsubsection{Picture Books and Light Reading}

Observations revealed that CL3 had few picture books, but CL4 had more than 400 selected picture books. While the DPL in CL3 struggled to use the public library budget to support the MOE's early literacy project (see 7.2.3.1), CL4 had advanced with BSP training sponsored by numerous organisations. However, the centre did not provide any books. It was fortunate that they had received a substantial picture book collection from a charity and villagers were eager to use it. All participants in Case 2 were of the view that most parents and teachers in urban areas and semi-urban areas like their village, were now open to RfP.

\subsubsection{Reading Activities}

Further to reading to young children and the newspaper discussion previously presented which highlights the crucial role of CLs under ONIE, in this section theoretical aspects of reading activities relating to control over book choice, the affective aspects of reading activities in CLs, circulation services, and rewards and accountability are discussed.

\subsubsection{Control over book choice and the affective aspects of reading activities in CLs}

The case showed a liberal perspective towards book choice by all participants. In CL4, the SOT gave selection rights to villagers. A reading volunteer talked about book selection: "We also got a budget from the owner of a large department store to select books from the regional book fair at that department store. We brought our young and old villagers with us to select books that suit their interests" (CL4 Jira).

As mentioned in 7.3.1.2, most participants held the view that in general most villagers and kindergarten teachers in the villages were strongly knowledge-oriented, so they usually acquired Thai $A B C$ books for young children, therefore this age group was possibly the one that had limited book choices to support their literacy development. 
An ONIE Report found in CL3 provided more details of the affective aspect of reading activities in CLs. It portrayed the pleasant atmosphere in cooking classes, craft sessions, and children's play activities which lead to RfP.

\subsubsection{Circulation services}

Book circulation is a major activity for CL4. However, there are few books in CL3, thus the DPL initiated a clippings service from newspapers subscribed to by the SBH. Thus villagers can keep, circulate, and read articles of interest to which she assigned one of three broad headings relevant for the village: agriculture, education, and health. The DPL talked about leisure activities which brought both pleasure and a better living, for example a women experimented with planting mango using the method she read from the newspaper clippings. Her comments supported the ONIE executive's assertion in 7.3.4.

In CL4, where there was an extensive collection of books, there was a self-circulation registration notebook in which the villagers noted the books they had borrowed. When they returned books to the library, the shopkeeper signed. However, as an informal organisation, they do not care if books are lost from the $\mathrm{CL}$. Regarding the nature of books lost from CLs, the leader in RfP found that serials and books in CLs were normally informally handed on from reader to reader. Thus she thought people take publications because they are interested in them, they take them back home to read and may forget to return them to the library.

The project that brought pride to $\mathrm{CL} 3$ is book distribution to other $\mathrm{CL}$ branches, the $\mathrm{ECD}$ centre, and elementary school teachers.

\subsubsection{Reward and Accountability}

All participants viewed reading as a reward in itself so did not see the need to give any extrinsic rewards to readers. However, accountability is a problem in the government system as mentioned by the DPL in section 7.2.3.1. She mentioned that the district ONIE wants a high percentage of children that can read, but the SOT does not gather the necessary data for this MOE literacy policy. Thus she needed to estimate the numbers to satisfy the authorities. 


\subsubsection{Creation of New Readers}

When discussing the creation of new readers, the leaders in Case 2 agreed with the idea that investing in the creation of new readers is more important than investing in library buildings. However, she added that activities that have an affective element are most important for the creation of new readers, as mentioned in 7.3.2.2.

She also mentioned a shared activity between children: "There was an admirable reading activity here. After school, children would read together at the library. Older children would teach the younger ones" (CL 4 Tassanee). Participants agreed that the affective element is vital for the creation of new readers in the $\mathrm{CL}$ setting.

\subsubsection{Community Participation}

In both CLs the village heads are dedicated individuals who actively and continuously stimulate villagers to read for pleasure. In CL4, new titles in the CL would be announced via the village broadcasting system and participants asserted that community bonding was strong especially among the middle aged and older people who prefer to participate in village events collectively. The villagers always give their best efforts to welcome guests who regularly visit the CL. However, the reading volunteer mentioned that despite the fact that most of the villagers can afford books, the villagers are not ready to participate in acquiring new books. This shows the villagers' attitudes to book purchase and the sustainability of the CL which depends on support from other organisations, not self-reliance.

\subsubsection{Location}

All participants agreed that the location of CLs must be easy to access. However, in cases where the current location is not suitable, the villagers can find a better spot. Documents on the SBH Operation in 2015 and an ONIE letter in 2015 show that after the project had been operating in half the villages in the country, there were problems with a delay in the delivery of the daily newspapers, and serial subscriptions that did not conform with the community's selections. The letter listed three urgent actions, and one was a relocation from unsuitable houses to which SOTs/volunteers could not deliver the daily newspaper on a regular basis. 
According to participants, a good location also means an atmosphere that encourages people to have open collective conversations. The DPL mentioned: "The SBH must be a place that people get together to discuss local news. At the time of SBH establishment our country was in unrest so people wanted to read about it" (CL4 Lak).

However, an active SOT mentioned that reading material is more important than location. She affirmed that if ONIE can provide better materials, and she can encourage RfP without a permanent location. Participants mentioned the mobile library, but the ONIE mobile library usually comes to the village only once a year and this is not regular enough to support RfP.

\subsubsection{Key Individuals in RfP and Training}

In Case 2, the two leaders in RfP showed that they were passionate about RfP, were creative, and voluntarily devoted themselves to $\mathrm{CL}$ initiatives, even before the SBH project. They also had a strong view which parallels Krashen's, that rural people will want to read for pleasure if they are provided with books, as mentioned in 7.3.3.4. However, providing reading materials to CLs with no budget requires individuals with a high potential to cooperate. The example is the retired ONIE executive who had been in the position for a long time and had connections. Thus, she was able to collaborate with the provincial ONIE, an international charity, and a large department store to acquire books for CL4.

Participants in Case 2 noted the multiple roles of the SOTs in the community. Reading promotion for early childhood was added to their workload of providing non-formal education for school leavers and adults, as mentioned in 7.3.2.1. In addition, a SOT in CL4 commented that there are no clear guidelines on their roles and activities for most new projects ordered by ONIE. Therefore, it is difficult for SOTs to juggle work and achieve all required tasks. The power tension between them and the DPL, as mentioned in 7.2.3.1 and 7.6.1, shows SOTs' multiple roles.

An active SOT in CL3 talked about ONIE training for the early literacy policy, which had limited practical value. The trainers had not taken sample picture books to show the trainees. The leader in CL4 described the general ONIE reading volunteer training that briefly introduced them to the principle of activities design, similar to the work of an 
activity facilitator who can plan activities in their area. However, the training had been only 3 hours long so she thought ONIE should increase the training time and continue to train them.

The leader in CL4 mentioned that in the past SOTs were trained in customer service principles, adult learning, and cooperation. However, it was her experience that lately ONIE had not focused on those capabilities. Therefore, the SOT's working culture had changed. In the past, they had to live in the community but nowadays they travel to the community. She thought this is an essential factor affecting reading promotion in the rural areas as the SOTs did not know the community and its people as well.

In both CLs the officers are friendly, talkative middle-age women who are happy to volunteer for the community. A reading volunteer, another friendly middle-age woman talked about the interaction between the village reading volunteers and SOTs which needed to be based on friendliness, respect, and cohesiveness. A class division between the peasant/villagers (chao ban) who are less educated and the professionals (chao nai) who are well educated and superior can be seen from her perspective.

\footnotetext{
School teachers are different from SOTs... SOTs who work in this area are very nice, they are not arrogant like ordinary teachers. Teachers are normally proud of themselves because they have position and rank. Kitiya, our SOT, is very friendly. So, we dare to talk to her and like to work with her in every task... We are proud and happy that we are common people and she values and respects us... We are common villagers so we are modest... When she has regularly acted like we are equal both in the village and in other places, we are very happy to work with her. We help each other to operate the $\mathrm{CL}$ and have got the third runner-up prize for outstanding SBH for two consecutive years. I think the library staff member who got the first prize is a retired teacher. She looked arrogant and not friendly... (CL3 Jira).
}

Kitiya also mentioned that SOTs need to be approachable for villagers and equally respect them in return.

The passion and dedication of the DPL affects the success of CLs. The DPL in CL3 mentioned that after a new SOT was assigned to take care of CL3 instead of Thana, she found that he did not know how to engage in activities and was quite dull and religiously 
devout. Thus, she had to deal with everything by herself. In the future, it is likely that she will be less active with CL3 because it is not easy for her to work with the new SOT.

In $\mathrm{CL3}$, the DPL mentioned the uncommon practice in her district that the WA had trained the reading volunteers. Their training was more comprehensive than the general ONIE reading volunteer training.

\subsection{Summary}

The case provided deep understanding of ONIE's SBHs which have a long history as village reading rooms and was the largest $\mathrm{CL}$ project in the country. The retired ONIE executive and the DPL gave specific data on not only the two CLs but also the overall picture of $\mathrm{CL}$ operation in the country. They also reflected on the ability of people of different socioeconomic status to access books, and commented on some literacy traditions that hinder literacy development and RfP in Thai children.

Findings from Case 2 show that ONIE CLs had limited reading materials and this is one of the major factors affecting RfP in rural areas in the country. Key individuals, especially leaders in RfP, SOTs, need to have a strong passion and understanding of status to work with villagers.

The findings also show the interaction between the DPL and SOTs who are major figures in community reading promotion in the country. Their coordination defined the success of CLs and reading promotion in the area. 


\section{CHAPTER 8 Case Report 3 \\ CLs established by Health Promoters}

\subsection{Introduction}

Case 3 is comprised of analyses of CL5 and CL6, two independent CLs established by health promoters through their interests in healthy living, RfP, and early childhood development (ECD). Leaders in RfP in Case 3 passionately and persistently worked and inspired villagers and disadvantaged people in their areas through bookstart projects (BSP).

CL5 is located in a public health centre (PHC, under the Ministry of Public Health) in a distinctive Catholic district in a small province. The community had established a private nursery and private primary school during the previous generation. The fundamental characteristic of CL5 is the free distribution of picture books through a BSP, which was a result of the strong passion of the PHC director (Yada) who started the project without support from the PHC in 2008. In Thailand, PHCs are responsible for promoting healthy living in rural areas. The development of children aged $0-3$ is mostly overseen by PHCS and child development centres (CDCs - after 1999 transferred to be under the TAO, the Tambon (Sub- District) Administration Organisations). The success of CL5 was relied on a picture book author, the TAO, local teachers, and public health volunteers (VHVs). Other participants are Nida, a reading model, and Wanna, a school teacher who cooperated with Yada in a folklore project.

CL6 is a library in a health foundation and a nursery run by two protestant individuals (Naree and Nalinee) who primarily work as health communicators and use books as part of the NGO's activities to promote health matters. Since Happy Reading was established under the Decade of Reading agenda, and Happy Reading was a major supporter of projects in CLs from 2010, CL6 can be considered under the Decade of Reading policy. The $\mathrm{CL}$ has supplied books and training in rural and marginal communities in the city. CL6.1 is a mini case under CL6 that portrays another example of a project in a disadvantaged area of the town which community leaders' (Orawan and Pichitra) 
attempted to improve conditions for the people living there. After data collection, CL6.1 transformed its active movable book box project into a physical library.

\subsection{The Thai Reading Context}

\subsubsection{Literacy Problems}

At CL5, Wanna, a primary school teacher collaborated with Yada on an oral literature and book production project. The Thai language teacher said that there were literacy problems in the CL5 area, and a project run by seven volunteer youth "librarians" studying at junior high-school level (ages 13-15) helped the young ones who could not read. This strategy helped younger children who could not read start to know how to spell after three months of the project. The project was not a $100 \%$ success in terms of spelling, but all the children loved the picture books and were happy to be exposed to them. She also added that building reading skills in this way is ideal for early learners.

\subsubsection{National Reading Policy}

Most leaders in RfP in Case 3 mentioned reading policy in the broadest sense the reading culture. Despite indications that several aspects of the Thai literacy tradition hinder RfP, as presented in the later sections, they suggested that a reading culture can start to be built with this generation. The leader in CL6 discussed a reading culture entwined with Thai literacy tradition and the significance of the RfP concept to this vision.

In the Thai literacy tradition, they just force very young children to write by holding the children's hand. They do not know that reading to children is preparation for literacy development. So they only train the small children to write. Write their name first. Is it a part of the culture?... If it is..., it is an obstacle [for reading culture]. The culture is what we repeat over and over again. But the culture can be changed; it's up to us in this generation.

...if we want to promote reading, we should make it fun first. We have to try to make reading a happy moment, and everything else would follow. Actually, I think the government's policy, plan, and projects have to be reviewed because of your PhD study (CL6 Naree). 
Similarly, another leader in CL5 stressed that reading promoters need to spend a lot of energy on promoting new reading practices, for example reading to young children. She added that the Decade of Reading agenda would work in the long run if there were more parties involved to form a network. Most importantly, there must be a process to identify and create reading volunteers. "We have to find those who will not let go of things easily and those who are not only waiting for the policy from their boss. Because if we wait for the policy, it will never happen" (Yada CL5).

Most participants agreed that policy instability at both provincial and national levels greatly affects RfP. A leader in RfP in CL5 talked about the volatility and ego of Thai executives. This had led to the situation where CL5's BSP had been initially wellsupported by the former governor, but when the new governor changed his focus, support from the province was significantly reduced.

Project documents and leaflets revealed that CL6 was positively affected by the Decade of Reading agenda because their reading projects had been primarily supported by Happy Reading since 2010. The NGO at CL6 was also significantly involved with Happy Reading and had campaigned on informing villagers and the TAO that the TAO's allocated Tambon Health Protection Fund could be used to establish an Early Childhood Literacy Fund (ECLF). The documents identified that Happy Reading aimed to mobilise the ECLF to provide at least 3 picture books to every family with children aged 0 - 6 years and ECLF could be of great benefit to children in marginalised areas. However, a community leader said that there should be a more explicit national policy on ECLF. In CL6.1, the urban poor community members currently use their community-savings fund to provide books to mothers who give birth. Her comments reflect the marginalised situation in the urban poor community: "Naree said there is the policy, but, it has never reached us" (CL6.1 Orawan).

The last aspect of the National Reading Policy of relevance for this case is public awareness of ECD and RfP. Yada mentioned that before she started the BSP in 2010 even educated health promoters who worked in her PHC had not known about the value of reading to young children, and therefore uneducated villagers were unlikely to have this awareness. She contended that the government should invest more on publicity for ECD, 
mainly the importance of reading to young children. Another leader in CL6 supported the notion that villagers who are aware of RfP would buy books.

\subsubsection{MOE Policy}

\subsubsection{MOE Literacy Policy}

All participants commented that the new MOE literacy policy was too demanding and caused too much stress for school teachers. The teacher commented on the top-down policy design and implementation that the MOE should not put pressure on the teachers, and that MOE policy needs to be stable and concerned with practical CT language teaching in rural contexts. She added that literacy development in rural areas takes more time because when parents finish their work that requires an early morning start, they are too tired and do not have time to take care of their children, so most of the burden falls on the teachers. It is unrealistic to demand that $100 \%$ of students can be literate at grade 1 because students have different potential with reading. For some with learning difficulties, the teachers would work very hard to just help them to get through until grade 6 (age 12). After that, these students may not be able to study in the strict school system but they can further their education at the ONIE Centre.

She added that in the CL5 area, the students were luckier than in other villages because either their parents joined a BSP or they attended CDCs that had all joined BSPs. Thus, with sufficient picture books, at grade 1 these students were rather well-prepared for formal schooling.

\subsubsection{OBEC School Libraries}

CL5 demonstrated the close connection between the $\mathrm{CL}$ and the school library. The PHC director who initiated CL5 asked a school teacher to start the folktales project, and the project brought a distinguished award to the school library. Therefore, there is not a clear-cut distinction between CLs and school libraries in this Isan community. Details of the project are presented in 8.3.2.2.

Participants noted that nowadays teachers do not emphasise textbook reading, but school libraries in rural areas generally have very few books to read for pleasure. In contrast, in the urban area leaders in RfP in CL6 shared their experiences with effective 
primary school libraries. Nalinee described an activity that encouraged her to read many books for pleasure without caring about the extrinsic awards the project provided. Therefore, in this instance, reading activities at the school library enhanced the chances for children to read more. Naree pointed out that the school librarian is crucial for RfP because they can create reading promotion activities that can make the children love reading, become a communicator between the library and the parents, and lead the children to the world of freedom of RfP.

The school teacher in CL5 insisted that the government should provide a professional school librarian position for each primary school. She said that teacher-librarians who graduated with a degree other than LIS do not know how to classify books and are not good with technology. Thus, they cannot manage the library properly and do not feel comfortable teaching volunteer students.

\subsubsection{ONIE Public Libraries and ONIE Mobile Library}

Participants in Case 3 agreed that public libraries generally could not support RfP. Only Nalinee who lived close to the city and was confident found the public library a good place to spend leisure time. However, most participants said that the formality and traditionally silent, unfriendly atmosphere of the public library deters rural people from approaching librarians and accessing books. Details of the impact of the atmosphere are presented in 8.3.2.1.

Participants in Case 3 agreed that the ONIE mobile library played vital roles in RfP in rural areas. However, leaders in both CLs commented that the playing of television programmes/videos in the mobile library caused a distraction for young children, particularly in relation to RfP.

\subsubsection{Coordination between Supporting Organisations}

Leaders in RfP agreed that networking between organisations is crucial for the promotion of RfP in communities. CL5 was a very good example of the possibilities of collaboration, which included coordination with a local agency, an artist, scholars, and doctors, and the leader in RfP expected to involve more parties in the future. Most importantly, CL5 showed how cooperation with the TAO greatly contributed to the 
success of the BSP. A leader in RfP in CL5 talked about mobilising public opinion to seek support both from her network and external organisations, and her strategy to progress the BSP and gain cooperation from the TAO.

\footnotetext{
We approach the TAO for financial support because we need [budget to print] books for children. The success of our initiative comes from our civil society consciousness. We know that that Bookstart is valuable and brings happiness to both children and parents...If we continuously do it and we never let it go, the villagers will realize how important it is, and the TAO will surely cooperate because politicians want to get votes from the villagers. The TAO will give us some money to buy books and we need to arrange a ceremony to handover the books. Therefore, the TAO Chief Executive will gain face (popularity) (CL 5 Yada).
}

Similarly, the NGO in CL6 also focused on civic society activity but through different groups, for example, the Children's Assembly and Youth Reading Groups. Documents in CL6 showed that the NGO in CL6 was a very active reading promotor during 2010-2016. The projects were well-planned, and supported and monitored by Happy Reading. The NGO gained extensive experience and cooperated with agencies to expand its networks. The 2016 project was a continuation of those from 2014 and 2015 aimed at mobilising ECLF by raising awareness of the importance of promoting the ECD principles in local TAO Committees. The NGO hopes that more awareness will result in the greater funding that TAOs committee members provide to ECLFs in each community.

Leaders in RfP in both CLs agreed that any organisation could create a CL. However, a responsible party such as ONIE is needed, although ONIE still needs to coordinate more effectively with other organisations, (e.g., the TAO and the health promotors). A leader in RfP in CL6 suggested that the ONIE should discuss with the TAO the duplicated newspaper subscription problem in SBHs and cooperate to develop this project. She commented that since reading promotion started with 19,000 CDCs, which are currently under TAO supervision, if ONIE can cooperate with the TAO to operate CDCs, RfP could be promoted more efficiently. However, she understood the fact that ONIE's expertise is adult education and ONIE and the TAO are under different ministries, making it challenging to collaborate. 
Similarly, another leader in RfP in CL6 noted that ONIE has great potential to support RfP in CLs because there are a lot of books in public libraries under ONIE. Yada also confirmed that her award-winning CL was not related to any ONIE CL projects. ONIE provided, only a box of books for adults and did not provide any books for children, as well as no followup was made. When she tried to acquire more books, an ONIE officer in her area told her that ONIE's administration policy has generally changed, for example, executive change, and each director has different preferences and/or priorities.

\subsubsection{Book Market}

All participants in Case 3 agreed that the shortage of books and bookstores in rural areas greatly hinders RfP. The only books available for children in the area were Thai $A B C$ books in local food markets. Therefore, participants agreed that the creation of BSPs and then CLs in Case 3 generated change in the communities. A major factor that supported the BSP and encouraged RfP in CL5 was an author who gave the copyright of his picture books to the province. As a result, CL5 can publish them with a cheap price, only 25 baht (1 NZD) per copy, and these books are used as giveaways.

In CL6, leaders in RfP, found that the solutions for villagers to acquire books were telling them to buy books in the city, using printed materials with photos for storytelling, and teaching villagers to make handmade picture books for their children. A leader mentioned her training in making picture books in Bangkok run by Happy Reading, which she found very useful for rural CLs.

Even though many participants agreed that books are costly in Thailand and are luxury items for villagers, as they observed, villagers could afford books if they understood the importance. CL5 showed that informed villagers could afford picture books from stores in the city. Yada (CL5), talked about book ownership in her BSP. "In this area, the purchasing of a book is like Nida's case, that the villagers buy the books themselves. It is not beyond their limit. But some families are impoverished, and we need to help...."

Nida's comments confirm Yada's assertion. It portrays a paradox between an informed and uninformed villager, and the individual's ability to access books.

.... woman was told by us (VHVs) to read picture books to her child so that her child will not be a slow learner. She said to me, "Sister, I have no books." Her husband is a teacher, 
but she still has no books! So I advised her that the PHC had some distributed books for free and she could take the books from there (CL5 Nida).

Yada not only acquired books for young children but also managed to subscribe to newspapers and find books for parents. She asserted that "If there were books, the villagers would read. They read everything, not only the books we chose for them" (CL5 Yada). The case also confirms Krashen's assertion about book access; that when attractive and appropriate books are provided, people read for pleasure. The theme of book access through BSPs is presented further in 8.3.1.3.

\subsection{Thai Cultural and Social Factors}

\subsubsection{Literacy Traditions}

\subsubsection{Teacher-based Learning Style and Home-School Partnerships}

All participants in Case 3 strongly agreed that the traditional teacher-based learning style and the lack of home-school partnerships negatively affected literacy development. Rural families mostly leave the literacy development burden to teachers when children go to school at six, though participants viewed that is too late to promote RfP. However, in CL5, Nida, who defined herself as "a strange villager", was serious about home-school partnerships. Her outstanding diligence and parenting that she gave to her grandchildren was confirmed by all participants in CL5. She was critical of the teacher-based learning style, and the obedience that Thai students traditionally practice by not challenging teachers.

In CL6, participants noted that the learning style and authoritarian mind-set in teachers still exist, and it is harder to change the teachers than the parents. Therefore, the NGO's projects, evidently in their project proposals submitted to Happy Reading, focused on parenting courses which aim to raise awareness of, and provide techniques for encouraging, RfP. A leader commented that a number of Thai teachers still hold the ancient traditions that they only teach, and the children must memorise what they teach and be obedient.

Overall, participants agreed that the Thai learning tradition has greatly discouraged RfP because it does not support personal preferences and critical thinking. An interviewee 
stated that the word 'teacher' connotes superior power, which creates stress and power distance. Participants agreed that these traditions need to be changed. The introduction of Totto-chan, as discussed in 6.6.3, had inspired many leaders because adults in the book respect small children and encourage student-based learning, the core of RfP.

As mentioned by Nida in 8.2.5, teachers are considered the educated class in rural areas, and participants agreed that in the teacher-based learning style they could positively encourage RfP by being role models. However, most participants asserted that Thai teachers are not readers. A leader in RfP suggested that it is possible for the teachers to become a model for RfP, but it should be introduced at the policy level. She questioned: "How to get the teachers to re-think about reading promotion. What should we do so that this matter is not a burden on the librarian only?" (CL6 Naree). There was such a case in which a former school administrator in CL5 appreciated reading. He supported Yada and Wanna's folktale project and RfP by allocating 30,000 baht for the library to purchase picture books, folktales and children's books for different age ranges.

In terms of a nursery caregiver, who in Thai can also be called as "teacher", Nalinee, the nursery owner in CL6 spoke at length about her decision to use the word "home" to describe her nursery, not "school" the word that nurseries commonly use. This is because she wants to give love to children by not being a teacher (a superior figure in Thai culture).

\subsubsection{Knowledge-Oriented Mind-Set}

All participants agreed that most Thai people still have a knowledge-oriented mind-set toward reading and learning, which greatly affected the take-up of RfP. Leaders in RfP in both CLs explained this mind-set.

The Thai perception of reading is to read for knowledge and so to become intelligent. Reading is for doing well in exams and also (academic) learning. Thais interpret learning in only the academic sense. In fact, self-learning can cover various activities, including RfP (CL5 Yada).

When we say "read," it becomes quite serious for Thai society. Just saying "read" makes us think of academic reading. We must precisely propose that RfP will lead to other benefits. We should let the people know that we should not focus on reading for 
academic purposes too much. There is the basis that reading promotion has to start from RfP (CL6 Naree).

Participants agreed that the shortage of books to read for pleasure in rural areas contributed to this mind-set in many adults living there.

Most participants noted that an overemphasis on literacy development, explicitly forcing children to write at a young age, can result in the opposite outcome. In addition to Naree's comments in 8.2.2, her sister elaborated on the forceful literacy practice at her nursery, where sometimes parents asked her when she could teach their children to write numbers.

...They are just three years old! I have to tell them that we have to prepare their physical readiness first. We have to let them play... now we need to train their muscles...

I have a niece... she had been forced to write since she was young. Every time she writes, her saliva always flows because she is very stressed. She suffers from literacy oppression. I told her mother not to do that with her, but she didn't listen to me. (CL 6 Nalinee).

In the urban area in CL6, Naree commented on the social value that focuses only on specific professional careers and fields of study requiring that children need to compete and read for examinations. This value can dramatically obstruct RfP.

The society should not force kids to study hard to be a doctor at their primary school! It is a distortion of learning, and it has created a lot of problem... it is extremely relevant to RfP. The children only read for exams." (CL6 Naree).

\subsubsection{Reading to Young Children}

The importance of reading to young children was the most prominent theme emerging from the analysis of interviews in Case 3 . However, this is a new practice which has just developed in Thailand. The leader in CL5 talked about the situation.

The academics of Thailand never mentioned about reading to young children as well... In fact, VHV has a prominent supporting role in promoting reading behaviour. However, the main role is the family. The government role is to increase awareness of the importance of reading. We should make the villagers realize that for early childhood development, the child's brain needs to be stimulated. It is their golden period (Yada CL5). 
Happy Reading identified the major factors affecting BSPs in Thailand: 1) the traditional beliefs of caregivers, and 2) book access for people in rural areas. Thus, this section presents these two factors.

The BSP in CL5 started in 2008 with little interest at the start due to parents' beliefs and their own limited ability to read. Yada (CL5) shared her experience with the BSP with help from the VHVs who attempted to persuade villagers in their homes.

\footnotetext{
We finally got nearly 100 families... My follow up research results showed that some families take just one month for babies to be addicted to books. Some babies do not sleep if there is no storytime... The key issue is the attitude of the parents. In the first phase of the project, most parents held the view that it's nonsensical to read a picture book to a newborn, they don't believe in its positive effects. However, when we organised events [for discussion], they understood. Also, many parents cannot read fluently so we provided a solution by asking the reading volunteers to distribute 20-30 books to the village and help read the books to the children Yada (CL5).
}

Nida confirmed Yada's comments that when the children started to get addicted to books, the parents/grandparents began to change their beliefs because they saw that their children have improved literacy development. An impressive case in CL5 that inspired many villagers was the clinically deaf-mute parents who were aware that their daughter would be mute because they were. Thus they joined the BSP and asked their neighbour, a 70-year-old lady, and cousins to read picture books to the child. VHVs supported by providing books to them and the girl was able to speak and understand quickly. In 2016, Nida estimated that about $60 \%$ of the people in villages were enthusiastic about reading to young children (ONIE in Case 2 estimated it at 25\%).

Nida talked about the literacy support she has given to her two grandchildren which also portrays the life of Isan workers who have to leave the children at home with grandparents.

I read to them ever since they were born. Two months after giving birth, my daughter needed to go back to work at a factory in another province... She sent the money to buy the best milk for the children, and I saved some money to buy picture books. I am worried that with no breastfeeding, the children will not be smart. I am a VHV, so I know that "The more you read, the more you know. The more you read, the smarter you are." 
I just keep reciting this. Sometimes, I am so tired but the sentences always echo in my head, so I endured it, and I kept on reading to the kids (CL5 Nida).

Dani, Nida's six-year-old grandchild, could read from age 5. The girl went with Yada to many provinces to share their experiences as reading models. Nida agreed that her grandchildren's behavioural development was very clearly affected by quality picture books.

Many documents in CL6 showed that the leaders were keen promoters of reading to young children. The Babies and their First Book (Happy Reading, n.d.) booklet supplied to CL6 by Happy Reading shows the benefits of reading to young children, and how parents can easily use picture books to stimulate and encourage their young children to develop and grow to their maximum potential. CL6 distributed the booklet and other materials to support their BSPs in the outer areas of the province. Documents found in CL6 from 2014-2016 showed its effort to promote ECLFs.

In CL6, after learning the work plan of Happy Reading, the leaders in RfP realised that reading could tremendously help pre-schoolers in their nursery. They started with removing televisions from the nursery and practicing storytelling time at least twice a day. Then, after noticing some changes in their children, parents could understand the necessity of a children's library for circulating picture books. A leader believed that the parents' awareness will significantly benefit children. "I want them to realise that if children have the opportunity to listen to good books; it will be their big inspiration when they grow up" (CL6 Nalinee).

The NGO in CL6 found that in the past, teachers, CDCs and other stakeholders did not know about their rights in Tambon Health Protection Fund. Therefore, there had not been any requests to use the fund for health promotion programmes for mothers and children which is one of the Tambon Health Protection Fund five target populations. In 2015 their project submitted to Happy Reading was on ECLF promotion at the broader district level, and in CDCs.

Yada found that storytime or time for rural people to read for pleasure is limited by their work; for example, villagers would read to their children in the late morning after they finished all their early morning work. This view is confirmed by Nida, and she added that 
despite the fact that some villagers have money, they did not spend it on books. Thus, BSP greatly supported book access and storytime.

As the nursery owner in CL6 observed during her 20 years in the nursery business, a great number of primary students could not read because no one read to them. Even though she has limited education herself, she understood from numerous Happy Reading training events that a caregiver should read to young children but not force them to be able to read or write. She had observed many CDCs both under SAOs and under schools, and found that in urban areas very few teachers read to young students in nurseries in Isan.

The observation of storytime at the nursery confirmed her comments. Her long interview highlights that reading to young children and child raising without physical punishment are still new practices in Thailand. Thus there is a need to spread modern ECD knowledge on these topics which benefits both literacy development and social behaviours.

\subsubsection{Learning Atmosphere}

\subsubsection{Low-Anxiety Atmosphere}

All leaders mentioned a low-anxiety atmosphere as an essential factor for RfP in CLs. The leader in CL5 talked about how traditional public libraries involved formal processes that make villagers feel awkward using the space.

In the past, we believed in the standard library system. However, when we did the project, we started to think that the formal library is out of reach for the villagers. The district public library is irrelevant to the villagers' lives, are there any people going there? If we enter the public library, we need to register for a library card. There was a need to have a photo as well. I would need to take a picture to get some books to read. I did not know what to wear to take a photo. The librarian also sits still so I did not know if he/she would welcome me into the library. When we did this project, we have a feeling that the library has to be easily accessible. There is no need for them to be a formal library. Just some book corners or the like are alright. Just let the people come in and see the books... they can quickly gain access to the books right away (CL 5 Yada).

The talk highlighted the CL's defining attraction: informality and approachability regarding the process, library workers, and library design. 
Naree, a progressive thinker on social structure and RfP in CL6, talked about authoritarianism in society that obstructs a desirable relaxed atmosphere because it creates high power-distance, and it hinders the freedom to choose, which is against the core of Krashen's FVR. Her sister identified that reading atmosphere is as important as books and people: "The general principles are to have an atmosphere that contributes to reading and the readers must also be ready to read or listen. Just let them read or listen without interfering or asking questions" (CL6 Nalinee).

She also mentioned that the leaders in extended projects in CL6.1 and other urban poor communities tried to create a relaxing atmosphere by giving their reading spaces attractive names, such as Bougainvillea and Marigold, identifying them as places where parents can come with their children to socialise and sit together to read. The observation and interviews in CL6.1 confirmed this.

\subsubsection{Sharing Reading and Oral Tradition}

Sharing reading can be seen in the literacy and book support activities in a collective society mentioned in the previous section and 8.3.1.3. Neighbours, cousins, and VHVs provide a natural support to people who need help building reading skill. Elderly neighbours and parents were reported to improve their reading ability gradually after joining the BSP. In CL5, when villagers exchanged books, the village had become a society in which people read together.

Another sharing aspect that brings happiness to rural communities was the oral literature project in school libraries mentioned in section 8.2.1. It inspired elementary school students to investigate, compile and write stories, and make books for the library. The PHC director aimed to make a connection point for children and the elderly in rural families and to create a social bond with the elderly. The books in the folklore project not only created family bonding and social bonding but were also well-received by students who used the school library.

The director also mentioned in relation to storytelling and reading, that music, talking, and listening is associated with RfP in an oral society. Similarly, in CL6 original bedtime stories told by participants' mothers were particularly imprinted in their minds and 
affected their love of stories and reading. Nalinee talked about her memories and the effect of oral communication on RfP.

\begin{abstract}
My mother told us both folklore and her newly created stories. I think storytelling is the RfP which is from our imagination or listening to music... Listening to music or radio allows us to use imagination. Even if we cannot see the alphabet, we can imagine.

I have heard the story of a writer who authored children's rhymes. He said that when he was young, he went to see musical folk plays. Those plays were poetic in nature, he did not mention books in relation to his early childhood. His literacy development was from beautiful poetic words in traditional plays...We use the oral tradition, which is part of our social capital, and try to engage the children. They are interested in it (CL6 Nalinee).
\end{abstract}

However, Nida also mentioned an aspect of the oral society that obstructs RfP. She commented that because most people love to talk, she needs to allocate her time for RfP. Sometimes when her friends knock on her door, she does not want to open as she needs some time to read.

\title{
8.3.3 Normative Culture and Feminine Society
}

The experience of most participants was that it takes time for villagers to become receptive to new ideas. Naree strongly asserted that the normative society makes it hard to change the old literacy tradition, and it is an important factor hindering RfP. Leaders reflected that the community opens up to changes if reading promoters raise awareness of training by presenting sufficient information about the impact of reading on literacy development.

The leader in RfP in CL5 said that the villagers feel embarrassed by what they consider to be progressive practice, and ridicule each other for it.

We need to create a new trend and make society as a whole see that it is right and they should not say taunting words to people who are different... a new mother who just gave birth to a newborn must have a friend to help. When we first did it, every one said they were afraid to talk to the child who could not speak yet because people would think that they were crazy. This CL helped a mother find some friends who also read books to their babies. There is nothing strange in the practice. (CL5 Yada). 
Another leader believed a collective Thai social element, reading together, can be done in a $\mathrm{CL}$ as a channel for community development, facilitating change in normative society.

[Without CLs] it is rare for reading in the community to start by itself. People are separated, and there is no communication between them. In fact, I think reading together can create a learning space for people to discuss issues. This is very important; discussion is a foundation for development. It can lead to many other projects. The villagers can think differently and accept the differences. They can do things differently (CL6 Naree).

The evidence highlights the impact of CLs in community development and civil society where people have free opinions. This indirectly affects RfP because Thai children can develop the confidence and skills to discuss and choose what they prefer to read, not only obey parents, as further discussed in 8.5.1.4 Light Reading.

\subsubsection{Attitudes towards RfP}

Evidence from previous sections has shown that all participants in Case 3 had positive attitudes towards RfP. Most participants identified their "forgetting themselves" or "lost in the book" experiences, which is allied with Krashen's FVR principles focusing on the affective aspects of RfP. In addition, despite the fact that Nida focused on her grandchildren's intelligence, she expressed her deep connection to RfP for the opposite reason, she read just for pure pleasure.

However, a leader also noted that RfP could be a misleading term in the Thai culture. Therefore, the right understanding about the variety of material to suit individuals' reading preference needs to be considered.

Thai people have an understanding of pleasure in only one dimension, which is 'fun without any knowledge'... when we say RfP, they will think of only comic books or celebrity magazines or the like. But I think RfP is for all types of books... We need to put the [individual's] pleasure in the lead... I mean that those books are what readers want to read the most, thus reading the books give happiness... I expect that this research will make society realize the value of RfP (CL6 Naree).

Most participants in Case 3 believed that the lack of awareness of the value of RfP, or not knowing that children can benefit from reading any books, affects RfP more than class and education. However, they observed that SES or purchasing power is an 
essential factor that hinders RfP, because poor people might not have money to buy books so they cannot access them. Therefore, in Case 3 raising awareness of the value of RfP and providing book access to people in poverty are keys to RfP in CLs.

As mentioned in 8.3.4, most participants thought that people with any level of education could have awareness of the value of RfP and some participants felt that parents and teachers nowadays no longer think that children should read only textbooks, although others disagreed. Due to her experiences as a teacher, Wanna was open to RfP; however, she did not know about other teachers.

\subsubsection{Media}

All participants viewed modern media as an obstacle to children reading. They found that if children are addicted to gadgets, they are not interested in anything else because modern IT devices simulate everything very close to reality. The nursery owner saw that children can become addicted to smartphones from a very young age, even those who are only 1 . Thus, even for pre-schoolers, she needs to negotiate with them. Participants found that adults, who are in fact also prone to fall into the temptation of media addiction, need to talk to their children so they know where and when they can use modern media, otherwise they are concerned that the addiction will lead to attention deficit, and communication, isolation, and literacy problems.

There are three types of media mentioned in Case 3: television, smartphones or tablet PCs, and social media. TV has been discussed previously as a distraction in the ONIE mobile library (8.2.3.3) and the nursery at CL6 (8.3.1.3). Some villagers viewed the smartphone as a substitute for storytime and books.

\footnotetext{
Many carers try to teach young kids to know about online media. The young kids can learn all these things. The adults praise the children on this, and they misinterpret that it helps the children to concentrate. This is quite worrisome (CL6 Naree).

...The kids of other families can play around with these gadgets and they are happy that their children can do it, but I don't agree. I am happier to see my grandchildren get addicted to books (CL5 Nida).
}

The understanding that the smartphone is smart media can result in the assumption that parents can leave aside storytimes. A leader in RfP recalled: "I gave the mother some 
picture books and asked if she read for her child or not. She asked why she needed to read to her child because he already knows many thing, and he can use a smartphone very well" (CL6 Nalinee).

However, Nida's story showed the power of RfP over an IT gadget. She talked about her daughter who wanted to provide a tablet PC to her young daughters.

She thinks that the children will be happy... I objected because I was afraid that my grandchildren wouldn't read books anymore. My daughter thinks that I'm too stingy... when I let my daughter buy it, I let my grandchild use the tablet. She played with it for two days, then she left the tablet and picked up a book to read as usual... (CL5 Nida).

These comments also suggest that migrant parents have good intentions but in fact can result in media addiction in young children. The teacher in CL5 observed the same good intentions; that some migrant parents give their children IT gadgets but they do not have the ECD knowledge and time to train their children to use them. Therefore "surfing for pleasure" and addiction affect their behaviours, RfP, and reading ability. She thought information literacy needs to be put in the primary school curriculum.

Leaders in CL6 positively used Facebook to promote new interesting books and the Book Bank activities at the Saturday market. In CL6.1 social media was indirectly benefiting reading activities in an urban poor community because photos of activities taken by the adults may satisfy a desire to post on Facebook. However, the children benefit from the activity overall because they are glad that adults give them attention and read nice picture books to them.

\subsubsection{Religions}

Religions played important roles in both CLs in Case 3. CL5 and CL6 are located in different parts of Isan, but revealed the influence of modern education and religious belief that positively affect RfP. In CL5 Nida's family were Christians of the Roman Catholic Church; therefore, she attended the Christian private school in the village. She had the opportunity to go to a large Christian school in the city and started to be a reader there at Grade 7 (age 13), but she left school the next year. However, she continued reading romances that friends from the Christian school brought her from the city. After 
she married she still read and she had two daughters and two grandchildren who love reading.

In CL6, 60 years ago Naree and Nalinee's grandfather sent their mother to a Missionary School. Christianity, education, and reading in their family influenced the liberal way in which both were raised. Even though seniors in her family were not middle class, they supported freedom, reading, and education. Naree, now a leader in RfP, recalled that: "Well, it originated from my grandpa. He liked to read... He was an expert on black magic who changed because he happened to read a pamphlet so he became interested in God and Christianity... Reading made him change his way of life". Similarly, her sister Nalinee commented that her inspiration for health and reading promotion was also nurtured by the family's social work and religious belief. She also found that books brought many positive changes to her life particularly when she has been afflicted.

\subsection{Isan Socioeconomic Influences}

\subsubsection{Education, Poverty, and Migration}

As mentioned in the previous section, most participants in Case 3 put their own personal priority on education and also lived in communities that value education. Therefore, their profile showed a group of educated Isan, either from formal or informal education. Nida and Nalinee, who both married young, were aware that they lacked training; thus, they needed informally to seek the opportunity to gain more knowledge via every channel. They finally became avid readers and leaders in RfP.

Naree was an example of an Isan person who turned disadvantage into her motivation to use libraries. She had learning difficulties at a young age but finished a bachelor's degree. She believed that her struggle, her poverty, and access to good libraries during school and college years were positive factors that inspired her love of reading. However, she saw the cycle of poverty and migration in the rural part of the province, suggesting that in some districts when the majority of the parents migrate, the educational dedication of male teenagers who live with their cousins significantly drops. They end up being school leavers and seasonal sugarcane-cutting labourers and cannot escape poverty. 
Educational opportunities and poverty were not severe in CL5 because people in the village have an additional income from their cottage industries. However, almost $80 \%$ of young people still migrated to work in other places. Therefore, large numbers of children were left with their relatives in the village and it was up to them to raise the children.

The findings from both CLs show that family literacy and book ownership was low in both areas. Yada's survey showed that before the BSP, every household had TVs but not books for RfP. Therefore, in a low-income family where there are no books for RfP, or there are uninterested caregivers, other people need to be role models for the children, for example, teachers and adults in the community.

Findings from this case show that family members are the influential factor on RfP. Leaders in both CLs believed that child-rearing that gives freedom will nurture RfP and this is up to the caregivers of the children. Children raised by their grandparents can become quite smart, as Nida's case in CL5 demonstrates. Her natural reading behaviour directly affected her daughters' and grandchildren's academic achievements.

\subsubsection{Isan identity}

Participants in Case 3 said that the stereotypical image of Isan people is poor. "The images of being Isan are, first, they are funny clowns; second, they are destitute; third, they are naïve and gullible" (CL6 Naree). They may also lack confidence when entering formal public spaces because of their accents, but most participants in the case did not think it obstructs self-development or affects RfP, which is different from worldviews in other cases.

However, one leader commented on Isan identity as an important indirect factor affecting RfP. The stereotyping and suppression of Isan had been an approach the central government used to gather resources, for example huge amounts of tax, she said. However, the government gives little back and asserts its legitimacy to govern the Isan people. She added that this widens inequality between the regions and has resulted in extremely polarised politics. This indirectly affects RfP because the unequal (i.e. lesser) educational resources allocated to rural Isan leads to poor quality schools, teachers, school libraries, and reading attainment, as mentioned in 3.2.1.3. 


\subsubsection{Isan Language}

Most participants agreed that speaking Isan at home did not affect CT because elementary school teachers assisted both Isan and Khmer ethnic children by speaking both $\mathrm{CT}$ and their mother tongue languages in lower classes (grade 1 and 2). After that, these children can gradually adjust themselves to CT automatically. The primary school teacher confirmed this teaching strategy would draw teachers close to the children and help increase their self-esteem and literacy development.

A mother who did not use the local language with her children found that children's brains have the ability to be multilingual. However, she concerned that if her children cannot speak CT properly or they can speak only CT they may lose the chance to be multilingual.

\subsection{Community Library Factors}

\subsubsection{Reading Materials}

Reading materials have been mentioned throughout the report because participants believed that the primary function of a $\mathrm{CL}$ is to facilitate book access, particularly for children. It is one of four major factors affecting RfP that Yada, a leader in CL5 mentioned: 1) creating opportunities for discussion, 2) book provision (with workers to distribute the books), 3) continuity, and 4) public awareness. Similarly, Naree in CL 6 identified it as one of three major factors: 1) books, 2) reading promoters, and 3) atmosphere.

\subsubsection{Responsiveness to Local Needs}

Book access in rural areas is a prominent theme frequently mentioned in the previous sections. In both CLs, leaders in RfP contended that they encouraged villagers to have more interest in reading but books were scarce. Participants in the case firmly believed that the saying "Thai people do not like reading" is not true. To increase book access, starting informal CLs is the answer, they suggested 
Participants agreed that communication could inspire and effectively promote reading to children so the government needs to find strategies to reduce book prices that currently block disadvantaged people from accessing books.

\subsubsection{Book addiction, Variety, and Quality}

Most participants mentioned book addiction, suggesting that a variety of picture books in BSPs and a variety of books supplied to all age ranges through CLs is essential. Yada found that her initial book rotation between families did not work because newborn babies were getting addicted to reading quickly. They became possessive of a particular book they liked. She explained her solution to the issue found in the BSP in CL5; that is, she decided to give one favourite book to the child. At present, her BSP gives three books to the mothers as newborn presents.

She also talked about the confessions of villagers who took books from the $\mathrm{CL}$, which she did not mind, and about seeking support from SAO and the authors to print picture books, as mentioned in 8.2.5.

Yada asserted that a wide variety and a vast number of books are essential. In CL5 where the whole village is open-minded about reading, as Nida observed, many villagers exchange the picture books provided at VHVs' places very often.

In CL6, leaders agreed that variety is vital for RfP and books should not be restricted to only selected titles and not be of just one type. Naree noted that although her mother bought her low-quality material, its variety supported RfP.

\footnotetext{
... 40 years ago, when I was in my primary school, my mom would buy me the cheap one-baht comics to read every day. My mom did not know that what she did was reading promotion. The one-baht graphic novels were printed on cheap proof paper and were widely available at that time, even at fresh food markets... The good thing about reading this comic was that it was challenging. I thought there could have been other fun titles. I had to find out more entertaining titles, and those cheap comics made me read a lot from childhood (CL6 Naree).
}

She added that books in CLs have to be diversified to fit all age ranges. She stressed that reading promotion has to start from RfP with a great variety of books.

\subsubsection{Picture Books and Book Donations}


Picture books are mentioned in the previous section, focusing on their importance and usage. In this section additional aspects of picture book selection and acquisition are added to give insight into both CLs. Yada talked about the selection process in CL5, saying that she realised that there is a list of 100 recommended books for Thai children (Chiengkul et al., 2000) but she decided to take the children to select the books in her BSP. She found that "...they selected the books that they could read. Not so many words, with big characters, and colourful illustrations. Actually, their criteria are simply according to the theory!" (CL5 Yada). Similarly, Nida and the elderly who read to a girl with deaf-mute parents choose books using similar principles, which allies with Krashen's FVR.

Case 3 revealed the outstanding abilities of both CLs in picture book acquisition and distribution. In CL5, in the rural area where villagers do not have picture books, Yada did not accept book donations for her BSP and distributed published picture books for free, as mentioned in 8.2.5. In CL6, Naree, who initiated the Book Bank Project in an urban area, received book donations. She saw that the variety of books donated provided choice. The Book Bank also accepted financial/cash donations and bought new books for rotation. Such new books are primarily children's books that they regularly bring to the organic market on Saturday for parents and children to read at the Book Bank spot.

\subsubsection{Light Reading}

As mentioned previously, most of the participants have a positive attitude towards RfP and light reading. This section presents additional information on two specific genres; comics, and novels. As Yada observed, in CL5 since there were no circulation systems, these popular genres came in and disappeared very quickly from the CL. "Not a single one [of Conan: the detective series] is left, the novels are also missing. As you have questioned me that if it is true that the villagers do not read, I would say it is not true" (CL5 Yada).

20-30 years ago some participants experienced an upbringing in which parents had control over light reading materials, for example novels. In contrast, Naree and Nalinee had been supported to read the one baht graphic novels which contain romance, murders, and ghostly scenes. 
Naree noticed that nowadays Thai parents are likely to be reluctant to support light reading. She mentioned the conflict in parents' RfP practice, which shows how the knowledge-oriented mind-set has been applied to educating children.

\footnotetext{
The new generation of parents love to read for pleasure, but may not let their children read! My son has been to Roi Et province and took some comics with him. There was a girl there that borrowed the books from him. When the mother of the girl saw that, she reprimanded the girl. My son told me the story. I then asked him why he didn't say to the aunty the good things about reading comics. He said that the aunty did not listen at all. The girl was perfect in school, so the mother told her child not to read the comics. But my boy saw the mother sneak off with the book and read it. She forbids her girl from reading it, but she read it herself! This kind of power abuse can prohibit a child from reading comic books (CL6 Naree).
}

This example shows a belief that comics harm children so it is necessary to control reading choices for Thai children, especially good children who do well at school and generally want to please their parents by obeying them.

\subsubsection{Reading Activities}

All participants agreed that an individual's choice to select the books they like is essential for RfP, however many Thai parents from any background might discourage children to read for pleasure, as mentioned in the last section and 8.3.4.

The leader in CL5 stressed that an excellent rural library is an informal but all-inclusive one. $\mathrm{CL} 5$ is at the PHC, and there are other book hubs in VHVs' houses that serve as book repositories where the service provider (VHVs) can take books to distribute and give recommendations of books to villagers. At the same time, regular users can easily access these venues and exchange picture books without formal processes. Therefore, open circulation services and reading services through BSPs occurred at these sites. In CL6, books can be borrowed by parents who send their children to the nursery run by the NGO.

With a large budget granted by Happy Reading, there was a large variety of activities organised by the NGO which established CL6. They ranged from a two-day public campaign, Miracle of Reading Festival, and a radio programme on three days a week. However, the Book Bank may be the most outstanding activity involving urban community participation. It was an active hub for people in the urban area to gain access 
to versatile reading material. It provided book exchange, financial donations, support for disadvantaged areas, and a reading service for children. The original site in 2014 was in the urban area, and in 2015 it expanded to other districts. Naree talked about two primary locations.

\begin{abstract}
The activities are divided into two parts. At the organic market, we have established a point for where people can donate books. We also have a donation box in which people can donate money if they wish. Right now we have about 100,000 baht in the Book Bank Fund. With this money, each month, we can provide 1,500 baht per urban poor community...
\end{abstract}

\begin{abstract}
The second part of the activity is at the public park... I bring only children's books, mats and tables. I have a poster explaining how reading for children is good, but I do not tell people there who I am. They also do not ask me. They just sit and read and then they get accustomed to it. I think if there are a lot of reading spaces like this, it will be terrific. When children go to the park with their parents, they will see books (CL6 Naree).
\end{abstract}

All participants agreed that the creation of new readers is crucial, because when the readers read, they are influencing others and even if there are no books, they will try to find them. However, in disadvantaged areas, leaders viewed it as equally vital to provide more books because even after instances of training, the eagerness to read will disappear if there are no books to read. Yada (CL5) asserted that book provision links with other community reading activities: "the result of the training is fast and clearly seen if there is book provision... when they go back to their places, they can read and exchange the books with one another later."

Throughout the report, all participants showed that sharing reading, Isan folklore storytelling, and reading to young children generate the affective aspect. Pride is particularly important for older children and adults who support the literacy development of younger ones.

Freedom is an essential element previously mentioned by Naree in 8.4.2 and a teacher mentioned the balance between freedom and accountability in school reading schemes. This allies with Krashen's "forgetting" principle in FVR and time provision in SSR.

I think a reading report is right in a way that it works for a short reading. However, if we want to instil the reading habit, we need to give freedom... I think there has to be 
the activity for children to read freely and regularly. Even a little amount of time will help them to absorb the love of reading naturally. Any writing created after reading needs to be in a creative and free manner... I think this way is more effective than a book report (CL5 Wanna).

All participants appreciated the intrinsic rewards which came from RfP. Participants mentioned direct encouragement, for example compliments are needed to support affective feelings. "From their facial expressions, they are very proud of themselves. Isan people do not like to praise their children because they are afraid that the children will be spoiled, we need to do this more often" (CL6.1 Pichitra). However, the leader mentioned that in the urban poor area where children do not have enough food to eat, she also created reading activities and provided food, snacks, and drinks for children as rewards.

\subsubsection{Community Participation}

Throughout the report, CL5 participants revealed that with Yada's firm commitment, the $\mathrm{CL}$ received financial support from local agencies, the TAO, and labour support from VHVs and volunteers from the ONIE. As the PHC, CL5, and schools in the community were mutually participating in the BSP, joint projects and resource sharing had been common practice. Since the school library had picture books, it also distributed them to the local CDC. Similarly, community participation is identified by the community leader in CL6.1 as a tool to build a sense of ownership, a mechanism to ensure sustainability. However, leaders in CL6 and CL6.1 found that community participation has grown slowly in urban poor areas where people need to prioritise making a living rather than their leisure activities.

\subsubsection{Location}

Most participants agreed that a $\mathrm{CL}$ might not need a building, which can be in many forms but needs to be easily accessible as mentioned in 8.3.2.1 and 8.5.2. The leader in CL6 added that reading spaces are not necessarily the luxury libraries. It is equally important to have a good library collection and have people acting as librarians who bring books and a circulation service to the users in the communities, especially children who are usually eager to see new books. 
Although most leaders support the idea of building readers before building a library, one leader suggested that having a physical library is better because showing the variety of books will help provide choices for readers. Reading behaviours and civic involvement are two elements necessary for CL establishment in residential locations. "It can be in somebody's home, and a group of volunteers who love to read is needed. Then they would find ways to acquire books" (CL6 Nalinee).

CL6.1 started from books in a box that Happy Reading supported later with more books. The box is called the Traveling Book Box. The portable library can be transformed into a library in open spaces every weekend. CL6.1 was popular among children who came to join storytimes and other activities that the coordinator in each community created. VHVs or volunteers in the community lead children in singing, playing, dancing and reading. CL6.1 provided the only dedicated area for children in the community. A leader talked at length about a most extreme case when parents stopped the education of their daughter to make her take care of three younger siblings. The leader's passion for helping the girl made CL6.1 not only a reading space but a place to support educational opportunity and teach life skills for children in need

\subsubsection{Key Individuals and Training}

As mentioned in 8.3.3, it takes time for villagers to become open to new ideas; health promoters who initiated and promoted RfP in Case 3 needed to give consistent support to the BSP and RfP. In CL5, Yada's passion for reading led her to secure grants from the Thailand Research Fund for her Master's study. She felt under-appreciated but the outstanding work she had done had been recognized by Thailand Research Fund as an exceptional research project in 2009. The Thailand Research Fund webpage added depth to the interview, noting in its key findings that after the introduction of the BSP there were fundamental changes in children, parents/caregivers, and teachers. The researchers and participants learned that the focus needs to be on young children so they get addicted to books quickly.

Further to the strategic thinking presented in 8.2.4., Yada talked about her passion, commitment, and indirect contribution to her public health work. 
...the BSP is something I cannot give up... We know that it is beneficial and brings happiness to both children and parents... As a public health professional, I was criticised for my topic and the faculty I choose to study in... Some people asked me, what do you do this for, your job has a lot of KPIs to meet. Are you crazy? What we are doing now has never been appreciated by my bosses and my colleagues... However, reading promotion contributes to the Ministry of Public Health work such as child development. The pregnant mother will come for pregnancy care earlier if she knows it affects her child (CL5 Yada).

In CL6, Naree's and Nalinee's inspiration stemmed from family and Happy Reading as mentioned in 8.3.6 and 8.5.1.2. They were very strong leaders because Naree clearly understood the challenge presented by Thai literacy traditions. She used them to propel her 4-year BSP and Book Bank projects initiated by the support from Happy Reading: "I understood that the environment of culture and tradition does not support reading promotion among children. If I can work past this obstacle, our work will be easy" (CL6 Naree). Naree talked about reading models who were trained by Nalinee to show how they read picture books during training events. It amazed everyone that a child aged 23 could recount a story although he/she did not know the alphabet.

With support from Happy Reading, leaders in CL6 were frequently trained in Bangkok, and they were well-informed leaders who can train others on a regular basis. Leaders mentioned that even though some training was not new to them, the information allowed them to have more confidence in their practice with children in their nursery and the training of children and parents. Participants in both CLs reflected that local leaders who initiated new ideas, arranged some training and led people to practice them, brought about positive change and the success of RfP in CLs.

\subsubsection{VHVs}

VHVs are the most crucial reading promoters in this case as mentioned by Yada throughout the case report. According to the Ministry of Public Health public policy, a pregnant mother comes to the hospital for care at local PHCs during the first 12 weeks of her pregnancy. If the mothers know about reading to young children, they could prepare for their children's development. Therefore, PHCs are the contact points for RfP for newborn babies, not the CDC or schools. Observation confirmed that the volunteer 
VHVs who are usually local women aged around 50 actively support the BSP. Yada also mentioned that the project greatly supports not only children's literacy development but also adult literacy.

In contrast, in the CL6 area Naree mentioned that the director of their PHC did not care much about the BSP, which was one of the the Ministry of Public Health policies. In CL6.1 where Naree supported the book box, community leaders ran the $\mathrm{CL}$ with their own community money and trained young people who could then be reading leaders.

This supports Yada's assertion that a reading promotor is one who has a high volunteer spirit and firmly believes in the new reading practice; thus they can promote the practice without waiting for government policy. In addition, other important characteristics of reading promotors are being local people who are close to the villagers, are pragmatic, have sufficient knowledge, and have affective reading behaviour themselves.

\subsubsection{Training}

Although this case showed that the estimated percentage of people who read to? their children in both CLs was rather high, compared with $25 \%$ in Case 2, all leaders in RfP mentioned the need for continuous stimulation/training in rural areas. Participants suggested that the government should offer support to increase awareness about reading at the policy level and several successful reading promotion models should be communicated to the public, especially to families who have little access to reading materials. This type of communication can inspire and effectively promote reading to children by family members.

Findings in this case show that there are three steps in training in the BSP for family members, caregivers, and teachers: 1) communicating by organising a learning event to provide knowledge about ECD with reading models, 2) providing picture books for free, and 3) following up with a weekly home visit. Focusing on just the first of these, there is a need to involve community leaders and the people who act as reading models who were previous participants, because their reflections and experiences act as inspiration for new participants. In CL5 there were two annual events for each village. In CL6, the NGO expanded their activity on a large scale by organising a Book Worm family camp, empowering networking and social management, and supporting a creative media space 
for children and youth. The NGO initiated training to create 100 reading volunteer networks in schools, and over 30 civil society organisations in the province.

8.6 Chapter Summaryln summary, participants in Case 3 found that policies need to be re-considered to make the general Thai population cognisant of the benefits of reading to young children and RfP. Simultaneously, to generate the desired reading culture, the prevailing Thai literacy tradition needs to be changed and the national reading policy must be stabilised.

This case is exceptional in terms of the religious background and the people who established the libraries. The health promoters in Case 3 revealed that they had a strong passion and commitment to promoting RfP at the community level. Even though CL5 was located in a government office, it worked in a similar way to the NGO in CL6 because the leader proactively worked from her personal interest, not the Ministry of Public Health's policy. Yada always thought out of the box and did not care when people questioned her about her determination to pursue her BSP.

Similarly, the leaders in CL6 strongly believed in modern ECD principles, for example, child-based learning, freedom in child-rearing and book selection, non-authoritarianism, and literacy development. In addition, Naree's opinions focused on the Isan identity, and economic suppression which resulted in parents' migration and the cycle of poverty and low education. With strong support from Happy Reading, her work involved a variety of activities and was extended from the nursery in the NGO to urban poor communities and disadvantaged rural areas.

Participants asserted the importance of: 1) access to quality reading material; specifically picture books; 2) the provision of books to people in poverty; and 3) raising awareness in Thai people of all classes about book access issues. Both middle class and lower class people need to know about RfP so that they buy books for light reading and allow their children to read for pleasure.

The understanding of the importance of a low-anxiety atmosphere would also help reduce authoritarianism, strictness in school, and the knowledge-oriented mind-set in general. However, this case showed that more profound knowledge of ECD could radically change the way Thai parents and teachers adjust away from the traditional 
teacher-based learning style and increase home-school partnerships instead, thus positively affecting literacy development and RfP. 


\section{CHAPTER 9 Case Report 4 \\ CLs Established by Individuals}

\subsection{Introduction}

CL7 and CL8 were founded by two individuals whose education and experience was in broadcasting and health promotion. They empowered youth groups in their communities to promote reading in the nearby communities. These CLs needed permanent library volunteers to run the CLs efficiently since they operate in a similar way to childcare centres.

CL7 is located in a small province in Northern Isan from which most of the parents migrated to make money by selling lottery tickets in Bangkok and other provinces. Thus, most children in the area need to stay with their grandparents or relatives. The $\mathrm{CL}$ was formed as a youth club in 2010 and later registered as an NGO. Participants in the research were comprised of a leader in RfP who established the CL (Paradit), an early childhood teacher (Amara), and a female youth who formerly trained to run the CL's activities when she was in high school (Boonta). The collection was quite large with approximately 500 books, primarily supported by Happy Reading.

CL8 was a children's library in an evening food market in the city. Previously, the food sellers' children had had no space to go while their parents were busy with cooking and selling food until 11 p.m. The $\mathrm{CL}$ had been transformed from a communal dish cleaning room in 2008 by a municipal official who sought help from her personal social media networks. The $\mathrm{CL}$ was originally well established as a children's library with a large collection of selected picture books, technically supported by the WA. However, after eight years, the size of the collection had decreased because there had been no library officer for three years. The three participants were a female leader who had initiated the $\mathrm{CL}$ project (Aree), the former volunteer library officer (Dara), and a male who formally joined the CL's activities when he was a youth (Preecha). Preecha is a graduate and was a teacher when she participated in the study. 


\subsection{The Thai Reading Context}

\subsubsection{Literacy Problems}

Participants in both CLs perceived that some literacy problems in their areas could be improved by reading picture books to young children. In CL8, the library volunteer suggested that some kindergarten children aged 4 - 5 could read because they had been exposed to many storytime sessions at the $\mathrm{CL}$. Therefore, some could read to her. Those who could not read would recount the stories instead. She also noticed that elementary schools in the city tend to privilege English over Thai so most children could not read Thai fluently.

\subsubsection{National Reading Policy}

Both CLs were established before the Decade of Reading policy. However, they later received grants from Happy Reading, the programme under the policy. Both CLs also received support from other organizations (see 9.2.4). The leader in CL7 mentioned that grants from Happy Reading considerably facilitated his work. He commented that other reading agencies actively supported the Decade of Reading policy, for example, ONIE's $\mathrm{SBHs}$, however the outcome of the policy's application had not reached children. The early childhood teacher also mentioned that she had never heard of the reading policy and people in the community had received very little information about it.

The leader in CL8 said that after she established the CL, she received support from Happy Reading to run a radio programme about books/reading. However, after broadcasting for over a year she needed to stop as her father was having health problems and needed her support. She further added that before working on the CL8 project, she had never realised that the government supports reading because in her small town there are no bookstores and no policy on reading had reached it.

\subsubsection{MOE Policy}

\subsubsection{MOE Literacy Policy}

Participants saw that the MOE literacy policy, which changed from the phonics approach to using the whole language method, had caused problems for students in the last 
generation. This is because students would be able to remember words, but did not know how the words were constructed. They tend to know only the first letter of each word, thus they cannot read. Since no participants were involved with the new policy they did not comment much on this issue.

\subsubsection{OBEC School Libraries}

Leaders in both CLs said that most school libraries in their areas were encouraging children to read for pleasure by purchasing a wider variety of books than they had in the past and organising events in the libraries. However, a participant mentioned that at her school, only one group of students who enjoy a good relationship with the teacher librarian often visit the library.

The Happy Reading Project Report 2015 highlighted CL7's projects aimed at engaging parents, school teachers, and communities in reading promotion. The projects included book acquisition for CLs, school libraries, and the CDC at a local temple. In addition, there were ten book donation points in public areas in the city. The leader in CL7 added that his youth team trained students from 10 schools in storytelling with finger puppets, which helped school libraries to attract children to their school libraries.

\subsubsection{ONIE Public Libraries and ONIE Smart Book House}

Participants did not comment extensively on public libraries, but the leader in CL8 contended that it is vital to have collaboration between organisations in society to run non-traditional libraries for the public. She talked about the importance of a relaxing atmosphere and friendly librarians.

[if we] wait only for the government, the library would never be welcoming. The government libraries are boring, and the librarians are grumpy, even scary. But if various organisations are in cooperation to establish a library, it will become a comfortable library (CL8 Aree).

A youth in CL7 talked about ONIE Smart Book House because she was initially involved with the grand opening event of the SBH project in Bangkok. She was asked by a SOT to travel to attend the launch event ONIE organised for all SBHs. However, the event did not provide her with any understanding about the project. She saw that there were the newspapers and magazines available in the village for a year as a result of the project. 
Later, when the government stopped supplying the publications and there was no reading material supplied, the SBH at the village coffeehouse was closed.

\subsubsection{Coordination Between Supporting Organisations}

CL7 received support from several organisations, mainly from Happy Reading, but also the Children's Protection Fund and the provincial social welfare fund. However, Pradit, the leader in RfP in CL7, talked about getting some initial support from the village committee but inadequate local support at the upper level, the sub-district level. The Happy Reading Project Report 2015 highlighted CL7's projects involving collaboration between partners in the district and provincial levels and their role in raising awareness of Early Childhood Literacy Fund promotion and mobilising the Reading District Agenda. There were government agencies, the private sector, local government organisations, Happy Reading schools and 18 CDCs involved.

Similarly, CL8 received support from WA, the city council, and Happy Reading, as mentioned in 9.2.2. and in Aree's comments in 9.2.3.3. who said that it is vital to have collaboration to operate a new type of friendly library.

\subsubsection{Book Market}

In CL7 Amara, the early childhood teacher, who always borrows picture books from the $\mathrm{CL}$ to read to her students, was asked by Pradit to join a training course on handmade picture books. She can now produce picture books and is able to train others (see 9.5.1.5).

The fact that there are no bookstores in the small town where CL8 is located, as mentioned in 9.2.2, which was confirmed by a CL volunteer who also had no idea where parents could find children's books. Her comments also show that picture books are hard to find, even in the city area.

Parents always asked where these books were bought from. I told them that I did not know because Aree was a person who supplied the books to the library. If there were no books for children, especially picture books, fewer people would use the library. Children's books were exotic and so attracted children (CL8 Dara). 
Aree, the leader in CL8, thought that book availability and the dominant knowledgeoriented mind-set affected book access in her urban poor area. She noted that parents who are poor cannot buy books for their children but yet can buy other toys. She has never seen these parents buy their children books. "They may think that children already learn at school and attend tutorial classes, so a book is the last option to buy for children" (CL8 Aree). Similarly, the ECT in CL 7 confirmed this notion. As she observed, rural parents are more eager to buys toys than books for their children. She thought they were compensating for their desire to have those toys when they were young. "They think books are expensive and very few of them buy books for children" (CL 7 Amara).

Other participants in CL7 confirmed that even though books are still expensive, most villagers can now afford books, which can be seen in Boonta's case. She considered herself lower class but still purchased some books and often borrowed novels from her friends and the $\mathrm{CL}$.

\subsection{The Thai Cultural and Social Factors}

\subsubsection{Literacy Traditions}

\subsubsection{Teacher-based Learning Style and Home-School Partnerships}

Findings in CL7 show that most Thai teachers still practice the teacher-based learning style and authoritarianism. However, most participants agreed that nowadays when children are young, they obey teachers like in the past but their obedience declines when they are in their teenage years. This was because technological advancement allows them to use other channels to find knowledge. Boonta shared her view about teachers that is, she respects and follows the teachers because of their overall behaviour at the school, not because of their higher status.

The leader in CL7 talked about youths in his project, saying that their involvement in the CL's activities has transformed them into confident youths, which is uncommon for Isan students. However, they were questioned by their teachers who expected them to be less sure of themselves and be followers. "The school teachers perceived them as "stubborn children." Thus, the youths from our group have been closely watched" (CL7 
Pradit). Pradit found that when these youths entered university they were more confident than he was when he was young.

By contrast, in CL8, the leader said that the modern school curriculum focuses on childcentred learning. The teachers are facilitators who guide students and help them to search for more knowledge. She thought teachers still influence children, but probably not as much as in the past since students can search for knowledge themselves.

Concerning home-school partnerships, the leader in CL7 said that most parents in rural areas still believe that reading promotion is the school's responsibility. It is not the parent's duty to buy books. However, he tried to engage schools with his $\mathrm{CL}$, for example, supporting the ECT to attend the handmade picture book training course, then organising an exhibition of her work in 2014. Copies of picture books in the ECT project were distributed to other CDCs in the area.

In CL8, a youth participant, who joined during the initial stages of the project and was currently a teacher in a private school, talked about the collaboration of children, their parents and teachers and the tradition of memorising and reciting. "At primary school, children are trained to recite books before lunch to allow them to remember and be confident with speaking CT..." He linked speaking skill and reading to memorizing and knowledge of the alphabet system and writing, not RfP.

\subsubsection{Knowledge-Oriented Mind-set}

Most participants in Case 3 revealed that before learning about ECD and RfP in Happy Reading training sessions, they had the typical knowledge-oriented mind-set that promoted the idea that literacy development started from reciting and writing the alphabet. Since CL7 received regular grants from Happy Reading, the early childhood teacher in CL7 was invited to join several training sessions that, she said, tremendously opened her horizons. She talked about her mind-set shift and parents' traditional expectations.

Before attending the training for ECTs, I bought alphabet books to teach my children.

After the training, I realized that it's the wrong practice. Most Thai parents also do that and worry if their young children cannot write first. They would ask the ECT why the children cannot write. The parents still have traditional beliefs, so I advise them that at 
a young age, children will not be able to write. I suggest that they should buy picture books to read to their young children... If I didn't attend the training, I would also have the traditional belief. Now I always tell parents that it is not age appropriate for preschoolers to be forced to write... not every child can write their name. It is not uncommon (CL7 Amara).

Even the leader in CL7 initially had no idea about RfP when he started the CL. His comments portrayed his shifting mind-set from reading for knowledge to reading for pure enjoyment. The omission of memorisation is also allied with Krashen's FVR principle.

...After several years, I have learned that I shouldn't force children to explain what they get from the books. I think that it's enough if they are happy from reading... Moreover, it doesn't matter at all if they cannot memorise the story. I want them just to be satisfied. When people finish any books, those books have done their duty, and the answers are implicitly embedded in the books (CL7 Pradit).

He contended that most parents still have a knowledge-oriented mentality; thus they would support academic reading more than RfP. Boonta confirmed Pradit's assertion by mentioning that her parents supported buying books but discouraged RfP.

... [My parents] hid novels from me, I borrowed those books from the library, and even now, I haven't found some of them! My mother told me not to read novels; they are useless. She asked me why I don't read textbooks. However, lately, they have given up. I've seen my friends' parents from rural areas having the same attitude about RfP as my parents. My father supports me reading. However, it needs to be reading for academic purposes. When I go shopping with him, he always asks me why I don't buy some textbooks. I wonder whether he knows that all types of books are useful, not only textbooks. Comics and novels are also useful in many respects (CL7 Boonta).

She noticed that because she regularly reads for pleasure, she can capture the meaning in any book more quickly than her peers. She does not need to read much before exams, and she finished high school with a good grade. She thought education considerably affects one's attitude to RfP. She assumed that people who have a higher education then her parents would know more than those who have less education, however, the critical factor is knowledge about the positive effect of RfP on children.

In CL8 the leaders understand RfP, but some youths in the project only partly understand this approach. The document analysis shows that in TV programme footage recounting 
the success story of the $\mathrm{CL}$, the books displayed were selected picture books and children's books to read for pleasure. However, a volunteer youth said, "I want younger children to visit the library because every book is knowledge." The word 'knowledge' is stuck in her mind so she cannot express it by using other words that describe light reading or $\mathrm{RfP}$.

\subsubsection{Reading to Young Children}

All participants in Case 3 understood the impact of reading to young children on literacy development. CL7's leader found that children who are normally read to by older children are fast learners at school because they have a thesaurus in their brains. When they see the real alphabet system, they can learn quickly. The leader mentioned that the reading promotors need to work with the parents' perception of ECD and literacy development. Therefore, children will have parental role models and understand that reading is an important part of life. In the training, the leader used a reading model to show the positive outcome of reading to young children. Participants mentioned that reading models surprised teachers and parents when they read aloud at reading festivals. The early childhood teacher the thought that this is because those teachers have neither belief in the benefits of storytime and picture books nor sufficient knowledge about literacy development.

In CL8, it took time for food sellers to see that picture books helped improve their children's literacy development, school performance, and their lives. From a brief observation, people in the community were in close relationships with each other. Older children (aged 8-10) from one family looked after and played with young children from another family. Elementary school children used the library as their playground, as the library was the only space available for them in the market.

The former volunteer officer at CL8 thought that reading is based on the time allocation of each family rather than the family's SES. However, the nature of their career may affect the family's ability to read to the children. She identified that most of the food sellers in the market do not have time to take care of their children, but the children developed their literacy by using the $\mathrm{CL}$ while waiting for their parents until late at night. 
The leader mentioned that the attitude toward book purchase and RfP needs to change, so that parents will buy books to support RfP, and children can own some books. Most participants agreed that parents, caregivers and teachers are role models for children's reading behaviour.

\subsubsection{Learning Atmosphere}

\subsubsection{Low-Anxiety Atmosphere}

Participants agreed that a low-anxiety atmosphere is crucial for RfP. The leader in CL7 mentioned that when he changed the target group from older children to young children, he focused on an entertaining atmosphere, positive peer influence, positive reinforcement, and reward. He supported the children when they wanted to read material of their own choice. His ideas about RfP were mostly allied with Krashen's FVR, except for the use of rewards. Pradit gave both stationary as prizes, and food as rewards for children who joined reading activities.

The informal atmosphere is particularly important for people who perceive themselves as inferior. For example, although Boonta is a university student who studies in the city and has more confidence than ordinary Isan students (as mentioned in 9.3.1.1) she does not feel comfortable using formal public libraries. "As a university student, I don't dare to use the big public library in the district. It looks very formal. I feel too tense to use it. If I were a teacher, I would dare to use it" (CL7 Boonta). This shows Boona's belief that a teacher has high social status and that status affects library use. Her thinking is confirmed by the ECT who have a strong sense of inferiority.

I absolutely agree about the self-confidence issue of rural Isan people. We are modest rural people; thus we look at people who dare to use the public library and question why they are confident to enter there (CL7 Amara).

Similarly, the leader in CL8 agreed that social status greatly affected library use. She talked about the $\mathrm{CL}$ and the interaction with users who were beggars.

Those who are of middle class or high class are confident to walk into the library. Children who are beggars come to the $\mathrm{CL}$ to read books, but they are not confident in coming in the library. In fact, our CL is the only library in the city that child beggars dare 
to visit. When those who visited the library see me outside the library, they would greet me with joy and give me the beverage they have (CL8 Aree).

This portrays the flat relationship she nurtured in the $\mathrm{CL}$, that the beggars felt comfortable with her. She further mentioned that the friendliness of library staff was a major factor affecting user confidence in the library. In addition, she talked about the Thai traditional physical seating arrangements and freedom in the library that she had in mind.

\footnotetext{
The children enjoy reading books by sitting or even lying on the floor to read. There are low [Japanese] tables for reading or doing homework. However, most children like to lie to read books. We allow them to do anything in the library. When I was young, I didn't like the library because the librarians were always strict and reprimanded me, so I did not want to go to the library. I don't want the children to think like this, so I allow them to do anything they want (CL8 Aree).
}

The library volunteer thought that because the $\mathrm{CL}$ has a welcoming attitude, street children dare to visit the library and interact with other children of the same age. She talked about the storytime and the relaxed physical atmosphere in $\mathrm{CL}$ 8. Observation confirmed the welcoming atmosphere, for even when there was no library staff in the $\mathrm{CL}$, it was open and clean. Therefore, people of all ages leisurely drop by the $\mathrm{CL}$ when they go to buy food in the market.

\subsubsection{Sharing Reading}

Most participants had been influenced by sharing reading among friends. The leader in CL7 said that peer influence and reading together dramatically affect reading habits. $\mathrm{He}$ started to read after he saw his friends read the translation of My Sweet Orange Tree by José Mauro de Vasconcelos and crying while reading. He was curious to know why they cried. After reading it, he felt the same as his friends. The youth participant also talked about her sharing a reading experience of the translation of The Gideon Trilogy by Linda Buckley-Archer with a close friend. Both comments show that the translation of world classics and current popular books are available in the Thai market, and have been popular among groups of teen readers for some time. 
The Isan oral tradition, a form of story sharing, has been used to involve elders in in CL7. The leader talked about the folklore that is disappearing from the community when elders pass away.

...I wish the community to participate in library activities, so I've tried to find a project to support this aim. The examples are "Tales from Mothers" and "Gom Tales," which are Isan traditional stories. After the old guy who regularly told us tales at the CL passed away, I cannot find anyone to substitute him. No one remembers that folklore (CL7 Pradit).

This shows an effort to get community participation from all age groups and literacy levels. The elderly might not be able to write but can tell stories that have been preserved by the oral tradition.

\subsubsection{Normative Culture and Feminine Society}

In CL7 the leader in RfP said that the political context in 2010 and the normative culture had affected the CL's operation. He thought that at the peak of the Thai political crisis any positive activities engaging youth to "do good" had been suspected of having a hidden agenda to promote the middle-class or "Yellow Shirts." As most people in this area are lower class or "Red Shirts," the youth group was suspected of having financial support and a hidden agenda. The leader mentioned that some stakeholders in the community did not cooperate with the $\mathrm{CL}$. The most important opposition groups were high school teachers who did not support their students joining the $\mathrm{CL}$ activities, as mentioned in 9.3.1.1. The leader also added that the teachers not only perceived them as stubborn children who act as confident individuals, but also denigrated the youths' pride in trying to change society through leading the reading activities in nearby areas.

The leader also contended that the normative culture greatly affects the library operation in another respect, in that people do not want to work in the $\mathrm{CL}$ because the jobs do not look good, provide hard working conditions, and are not well paid.

Another hindering factor is the feminine characteristic of the society that does not admire people who act differently. In CL7, Pradit and Boonta talked about youths who were different from common Isan students. They were reluctant in their reading 
promotor roles because they felt they were not appreciated by the schools and their village communities.

In CL8 the library volunteer who volunteered for many tasks in the community said "I do not care what other people think about me. Some do not like me. But I think I do good deeds, and I do my best" (CL8 Dara). This strongly shows that she is an individual who does not hold the feminine characteristic that most villagers have. The leader in CL8 confirmed Dara's statements by talking about the timid nature of rural people and poor people in Isan society as presented in 9.3.2.1 and in 9.4.2. Their shyness to get involved with volunteer activities hindered RfP in both CLs.

\subsubsection{Attitudes towards RfP}

All participants in Case 4 have positive attitudes towards RfP, as mentioned previously throughout the report. They believed that interesting reading materials that hook readers to continue reading to the end greatly contributes to RfP.

Most agreed that despite low SES, positive attitudes towards RfP lead to more active reading behaviour and literacy development. In CL7, Amara talked about her childhood in terms of limited exposure to proper reading material. Since her family was poor, she started her love of reading from reading newspaper wrappings that came with her mother's local goods.

Most of the participants mentioned that it was challenging to change the attitude of parents toward RfP and attitudes toward book purchase. The leader in CL7 talked about how his training was helping to increase awareness amongst parents of the value of RfP, book purchasing, and better literacy development.

I think at present everyone can afford books, but it mostly depends on their attitude towards book purchase. Middle-class people may not buy books for their children because they think the children can read at schools. Most parents in rural areas still see reading promotion as the school's responsibility. It is not the parents' duty to buy books... However, I can see some change in rural parents. After several years of the parents' courses that I organised, I can see that many parents understand more about ECD. They believe that reading to young children affects their literacy development. I can see many cases. Some told me that when they go to the bookstore their children beg for books (CL7 Pradit). 
Some participants found that the critical factor is knowledge of the positive effects of RfP on children. A leader in CL8 mentioned that middle-class parents who are aware of the benefits of RfP do not force their children to study in the excessive mainstream tutorial classes. They support RfP by allowing their children to buy books that suit their interests.

\subsubsection{Media}

The leaders in both CLs have experience in media and broadcasting; thus media had been positively used to empower youth in Case 4. A report CL7 submitted to Happy Reading showed a variety of activities involving media channels in building social communication at the district level.

The leader in CL8 used the social media platform Hi5 to gain knowledge about library organisation. She posted on $\mathrm{Hi} 5$ about this issue, and her social media friend recommended she visit a WA children's library in another province. She also gained supporting funds from friends on Hi5.

She further commented that nowadays reading had dramatically changed because most people have smartphones. When people can access reading materials from the internet, digital reading is essential. Smartphones had also changed engagement with the young Isan generation, since they are happy to take photos which they send to the $\mathrm{CL}$. Then they can then post the images on their social media.

In CL8 youth groups actively ran many fundraising activities to establish the CL. The library had their radio programme supported by Happy Reading, and several television programmes broadcast their success nationwide. The leader interviewed youths who attended the CL8 project and children using the library during the radio programme.

The ECT thought that the group that benefitted the most in CL7 young children up to age 12. After 12 , children tend to be more interested in IT gadgets. However, she thought that if these teenagers volunteer and get involved in reading activities for younger children, they will enjoy it and see the benefit of reading. Similarly, Boonta said that the problem of illiteracy may be the result of too much exposure to IT devices. According to her, many parents let their children be exposed to TV and smartphones. As a keen 
reader, she even does not read if she is on her smartphone. Facebook is too tempting for her, and she cannot stop following it. In CL8 the library volunteer explained that IT gadgets might be the reason why children nowadays are slow to read. As she observed, children are more interested in playing online games than reading.

\subsubsection{Religions}

The leader in CL8 commented that in Thailand it is hard to find people with a volunteer spirit. Most people still believe that merit can be gained only through religious activities or in Buddhist temples, however, Dara, a library volunteer, thought differently to the norm. She believed that her charity works outside the temple would also bring merit according to Buddhist beliefs.

\footnotetext{
I think that working for children or the elderly will gain a lot of merits. I always give help whenever I am asked to... I do several volunteer activities in the community such as being on a community committee, a community volunteer, and a VHV. Today, I also attended the VHV's meeting at the municipality (CL8 Dara).
}

She feels that some people in the community do not like her for her involvement in voluntary jobs, but she said she did not care what other people think about her.

\subsection{Isan Socioeconomic Influences}

\subsubsection{Education, Poverty, and Migration}

In the CL7 area, the leader and the early childhood teacher found that around $70 \%$ of parents needed to go work in other areas because they need cash to support their children's education. If they farm in the villages, it will be sufficient to eat, but they will not have spare money. Thus, many children are left with their grandparents, which negatively affects their literacy development. He observed that the children who live with their grandparents are also ignored concerning nutrition, therefore, sometimes this $\mathrm{CL}$ is similar to a childcare centre. The leader sometimes cooked and asked the children to join him. The ECT said that the grandparents normally have excuses, for example, illiteracy, and lack of time. Even though the child's parents send back money, some grandparents may be frugal and do not use it to nurture children. The leader observed that the children whose parents are at home and read picture books to them are 
different from those who are left with the grandparents; they would be able to read before they enter school.

Boonta's talk about her family situation portrays the life of an Isan girl who was lucky regarding her grandparents' care. They did not prohibit her from reading for pleasure, which was the opposite of her parents, as mentioned in 9.3.1.2. She observed that many children who stayed with their grandparents were not as lucky as she was. Large numbers of teenagers did not receive good care from their grandparents and they often had problems at schools, they quit their studies around age 14 , and then attended ONIE courses.

In the CL8 area, there were fewer children left behind. The library officer agreed that children who live with their grandparents tend to have many problems. She noticed that some of the children were upset as they saw some friends had parents with them. She always comforted them not to be upset because their grandparents also love them, and their parents have to work to make money for them.

The poverty in urban areas can be observed in CL8 where the food sellers' children have to wait for their parents until late at night. A former volunteer student talked about the situation before the $\mathrm{CL}$ was established: "These children had no place to go while waiting for their parents. Sometimes, they slept next to the store or bothered buyers. Thus, the idea to find space for the children was brought up" (CL8 Preecha). The leader mentioned that after the establishment of the library, the sellers had more understanding and saw that the CL changed their children's lives. Then they participated in the CL's activities.

\subsubsection{Isan identity}

Further to the issue of having the confidence to access public libraries, as mentioned in 9.3.2.1, most participants mentioned the timid and modest characteristics of rural Isan people who have low SES. The leader in CL7 talked about his inferiority complex when he studied in Bangkok, and that he thought that the Isan identity possibly hinders Isan people's personal development.

We normally accept that people in other regions stigmatize us. We think we are inferior to them. I had felt like that until entering a university in Bangkok. I realised that when I did university activities, I was capable of learning and I gradually gained self-confidence. 
Thus, I thought I could do whatever others do. I feel free. Why didn't I think like that before! I see that if Isan children could break the egg of our inferior feelings of being Isan, we will save a lot of time on personal development (CL7 Pradit).

The leader in CL8 supported the previous participant, and Dara's words in 9.3.3, that rural Isan people and poor people are reluctant to be visible, even when doing something good.

When we raise funds by playing music, some of those watching dare not go to put their money in the donation box. They prefer us to bring the box to them. If they have friends with them, they will be ok. This trait prevents us from doing many good things and breaking out of the social norm. It's probably because most Isan people value modesty. Thus, rural people don't want to reveal themselves publicly for any good deeds out of the traditional ways (CL8 Aree).

These traits not only greatly hinder public library access but also voluntary work, as mentioned in 9.3.9.

\subsubsection{Isan Language}

Most participants agreed that speaking Isan at home did not affect CT acquisition because the learning process using both languages at school gradually helps Isan students to acquire CT. However, participants stressed that being a reader helped them in relation to reading attainment and being proficient in $\mathrm{CT}$.

In addition, some participants mentioned that when Isan people speak the Isan language in their region, they are not shy, but if they are among a group of people from Bangkok or the Central region, they may feel embarrassed that they are Isan people who speak and eat differently from the central Thai people.

In contrast, confident and educated Isan participants in CL8 used Isan as a tool to show their identity, even in Bangkok. The youth volunteer mentioned that since she had been trained and had done a high number of activities, she is now confident and proud to be Isan. Therefore, she uses the Isan language in most situations, even when she is in Bangkok. The reading volunteer from the NGO in CL8 use CT only when it is necessary. 


\subsection{Community Library Factors}

\subsubsection{Reading Materials}

\subsubsection{Responsiveness to Local Needs}

The two communities in Case 4 did not report specific reading needs. Boonta (see 9.2.3.3) highlighted the importance of newspaper and magazines subscriptions for the $\mathrm{SBH}$. Other participants (see the next sections) also talked about the provision of book access for both adults and children.

\subsubsection{Variety, Quality}

In CL7 the observations showed that the collection was diverse, ranging from picture books and children's books, to translated novels for teenagers. The leader formed a youth group (aged 12-18) to organise reading activities for younger children. They gave villagers access to good books by bringing picture books to read for young children in deserted villages. He concluded that village children are delighted to see quality books, and they are eager to read.

\footnotetext{
In all, village children love to read; they simply run to the books when they see the collection we bring. The problem is not about rural children not reading; it's about no good books being distributed to them in the past (CL7 Pradit).
}

He also mentioned that reading promoters have to acquire various types of books so children can choose what they want to read, which is allied with Krashen's principles. Also, Pradit and Amara focused on book acquisition and having a person in the library who can recommend books to suit children's age ranges because children commonly do not know which books suit them. Therefore, CLs should have a person who can guide them.

In CL8 the former library volunteer identified reading material as the most crucial factor and noted that picture books bring excitement and nurture RfP. The $\mathrm{CL}$ welcomed donated books that also support adult reading because when people come to the market, adults prefer to read newspapers or magazines.

The footage from a TV programme showed that the library had initially been well established with an extensive collection of picture books and an excellent variety of 
quality children's literature, technically supported by WA. However, as the library was located an open area in a food market, after eight years $70 \%$ of the children's books were lost

\subsubsection{Book Addiction}

Participants mentioned book addiction; for example, the volunteer youth said, "I do read for pleasure, and when I'm lost in the book, I cannot hear what others say. I smile to myself" (CL7 Boonta). The same "lost in the book" happened to young children in CL8.

Children really like picture books because they have never seen books like this. They can stay in the library for a long time. Sometimes, they do not want to return to their homes. Some stay until 8 or 9 pm, and some remain until the library is closed (CL8 Dara).

Concerning children's favourite books, Amara, the teacher noted that the children would repeatedly bring their favourite one for early childhood teachers to read to them until they can remember it or can read by themselves.

\subsubsection{Picture Books}

Following the discussion on the provision of selected picture books, participants also talked about how book quality and variety fundamentally attract young children to read and thus nurture RfP. The former library volunteers who operated CL8 at the night market talked about the excitement of children over picture books. These reflections confirm the lack of picture books, which are crucial for engaging children with books and reading.

The pleasure that I have seen in young children is their appetite for picture books because there are no books like this both at home and at school. With their great contents and colourful pictures, children really like them... Parents said that it was good to have a library for children. They had never seen this kind of book provided for children to read or do activities (CL 8 Dara).

The ECT with handmade picture book training found that the books she made were attractive to the children. Her project engaged pre-schoolers' parents in the writing process and her sons in the illustrating process. They made copies of these picture books and distributed them to other nurseries in the area (details of the training are in 9.9.3). She also talked about children's natural approach to choosing picture books in that when 
they open the books, they will drop them if they don't like them. She can tell which books were the children's favourites by the condition of the books.

\subsubsection{Light Reading}

Most of the participants had a positive attitude towards light reading, especially the leaders who had established both CLs, as mentioned in 9.3.2.1. The leaders said that they would like to provide good books and wanted to invite children to read interesting books that they had not had the chance to touch and read when they were young themselves. The leader in CL7 talked about his mission to help teenagers find their favourite books and use books to help them through this critical period in their lives.

In the urban area, the leader in CL8 believed that nowadays, Thai people are more open to a wider variety of reading material.

\subsubsection{Book donation}

A report submitted by the NGO of CL7 to Happy Reading in 2015 presented activities the NGO organised with teacher and family involvement. The teachers were invited to share the lessons learned from the activities. Some of them assumed that villagers have no money to buy new books because each year parents are encouraged to give each student a storybook to donate to the school library, but mostly they donated old books that were not suitable for children.

CL7's campaign for book donations also found, on many occasions, that most of the books donated were old magazines, self-help books, Buddhist teachings, and English textbooks that were not suitable for young readers. In most meetings organised at the sub-district level during the Happy Reading project, there were discussions on how to acquire quality reading materials. Initially, CL7 provided 20,000 baht for each CDC to buy children's books.

The report also identified a new approach; a school committee is created to bring new books to the students. One teacher interviewed for the Happy Reading report said that in her school there was an idea to invite publishers to run a book fair for children and encourage parents to buy books to give to the monk leading the library/project as a form of merit making. 


\subsubsection{Reading Activities}

\subsubsection{Control over book choice, Affective aspect of reading activities in CLs, and}

Reward

In CL7 the leader said that children should start with whatever they like to read and adults should not force them to read. Regular activities in CL7 are a storytime given by youth volunteers and time for children to read by themselves. The CL provided a basket of selected picture books for them to choose from. The children will choose the book to read for their friends, and the leader or the youth who leads the activity will finally reward the children who are able to read to others at the end of the storytime.

He said comics and stories are acceptable. Children will gradually develop literacy so reading promoters can introduce more demanding materials later. At the same time, he focused on a fun atmosphere, positive peer influence, positive reinforcement, and reward, as mentioned in 9.3.2.1. He stressed that these strategies need to be done continuously otherwise other distractions that can easily divert children from reading. He talked about peer-influenced activities, competitive feeling, and rewards, which ignite reading interest in young learners.

Reading competitions and collecting points for each book read can be other peerinfluenced activities. When children see their friends read, they start to do the same thing. Collecting points for each book read can stimulate reading. Even though children read because they want the reward, it is an opportunity for them to see good books.

I also experimented to see whether, if there is no such activity, the children will go to read. I found that the children were still waiting for the reward to compete for. Thus, I thought that I cannot build the reading habit in children. However, I think I need to do these activities repeatedly to make books intrinsically enjoyable by children. I don't yet know at what point children will read without rewards, but instead read because of their real interest in books (CL7 Pradit).

The youth talked about the most popular monthly activity, "The Delighted Weekend." It was a half-day activity that encourages children to enjoy singing, dancing, cooking, and reading to their peers. A report submitted to Happy Reading shows that an average of 30 to 40 children joined the activity per session. The $\mathrm{CL}$ also provided lunch for them. The team and young volunteers created fun and friendly events, which were different 
every month. The parents took part in the planning meetings and brought young children to participate in the activities. There was a review of the lessons learned to create joint strategic indicators for the parents' networks in the area. In CL7, the leader focused on the affective aspect of reading allied with Krashen's FVR but extrinsic reward is not recommended by Krashen. However, in CL7 the target group was younger than that in Krashen's work and may be appropriate in this case. (FRV is neither for beginners nor advanced readers. Perhaps accountability and rewards are less restricting for beginners, who need some attraction to encourage them to join reading activities). In CL8 all participants highlighted the importance of freedom and choices (see 9.2.3.3 and 9.3.2.1).

\subsubsection{Circulation service and Accountability}

Circulation is a major activity for the CLs. In CL7, there was a record of the borrowed items. In CL8 the leader talked about the loose control of borrowing.

\footnotetext{
At the beginning, books were available to borrow. We have a library officer to deal with borrowing books. However, as most of the books were picture books with a few pages, they were rarely borrowed as the children can finish reading them at the library. So, not many people borrowed the books to read at home. There was no registration system for the book circulation, but everyone understood (CL8 Aree).
}

The former library officer also added that books were rotated and the old ones would be given as rewards for those participating in activities at the library. In terms of accountability, CL7 had regularly assessed their activities because they received grants from Happy Reading.

\subsubsection{Community Participation}

In both CLs, coordination between supporting organisations cannot be separated from community participation involving community leaders, as mentioned in 9.2.4. The reason the leader in $\mathrm{CL7}$ could not receive full support might come from the fact that he was away from this village for a long time so people neither recognised him nor considered him a local. He needed to ask his uncle to help him coordinate with community leaders. The village committee agreed with his project to build a library at the abandoned village nursery, but he did not gain further support from other local authorities, as mentioned previously. 
Another two participants also agreed that more participation from parents and local authorities would be beneficial for the $\mathrm{CL}$ and RfP. The youth volunteer in CL7 suggested that a more profound interconnection between organisations in the society and community leaders would change the mind-set and positively influence parents' attitudes towards RfP.

Cooperation in society is an essential factor affecting RfP. If schools, local government, and heads of the village support children and people to use this $\mathrm{CL}$, it will greatly affect people who work on RfP. Later it will gradually affect the parents' attitudes on RfP.

Generally, it's hard to change parents' attitudes on RfP. My parents usually discourage me when I discuss any topics with them. They still hold to the custom that they were born before me, so I need to obey them. However, the respected community leaders can influence them. Villagers need to be regularly encouraged on RfP, then they will agree with this new idea (CL7 Boonta).

In CL8, the leader and TV programme footage mentioned volunteer students in the city who had helped to raise funds by singing at the night market. Additional funding was obtained from the photo club in the province, and a second-hand clothes and doll sale. The leader in CL7 mentioned that the volunteer youths who pioneered the library have the sense of belonging to the CL. Youths in three villages nearby give great support to the $\mathrm{CL}$. He estimated that $20 \%$ of the youths in those areas volunteered at the CL. In CL8 the former youth volunteer agreed that if the library is established through collaboration, the sense of belonging will keep the $\mathrm{CL}$ going and people would take good care of it

\subsubsection{Location}

Since the two CLs are located in the centre of their communities, they are used regularly. The former library volunteer talked about the users who visit the library.

People from other sub-districts around the city also come to the library in the evening and at the weekend. When they and their family visit relatives, or come into the city or pick up their children at tutoring schools in the city, they stop by to have dinner at the night market before going home. So, they take their children to the library to relax after studying at tutoring schools (CL8 Dara). 
However, maintenance was a big problem for both CLs. The leader in CL7 mentioned that he needed help from local authorities to renovate the building. In CL8 the library space was well maintained, but the children's collection was much smaller than when it was established.

Leaders in both CLs agreed with the idea that the library could run a mobile library collection. However, it requires more attentive staff than a typical CL because the staff need to deliver the service regularly to communities.

\subsubsection{Reading Promoters and Training}

The CLs in Case 4 relied on volunteers. While CL7 was running with a new batch of youth volunteers, CL8 leaders mentioned that in the last three years there had been no volunteer officer in the library, so it was not as active as in the past. In the most active period, the evidence from TV footage showed the strong volunteer spirit of youth groups and volunteer officers engaged in fundraising and other activities. The RfP leader put a lot of effort into building volunteer spirit and civic involvement.

The portfolio of the NGO shows the history of the NGO that was formed from a youth group in 2009 in a small district with the intention of organising positive activities for the community. It started with a small group of young people in the community running a $\mathrm{CL}$ as a creative space and learning resource for children and youth. Youth volunteers were initially trained during the reading activities. Nowadays, the issues on which the NGO focuses are more diverse including drug use, sexual and reproductive health, and environmental issues. The Youth Volunteer Development Project is supported by the Municipality. In 2010 Happy Reading started to support a Volunteer to Read Project and continued it until 2017.

The portfolio also shows that the NGO run a number of youth camps, for example Youth Leadership Training in School, aimed to build a relationship between the NGO and schools, and hold activities to create readers (story-telling activities, making hand puppets from socks and telling stories with them). Young students were attracted to the events led by older youths. The NGO invited the youth leaders to bring their knowledge from their training in school to help the first graders at school to read and write. The NGO reported positive impressions and reflections from the participants who said that 
they had the chance to express themselves, join exciting activities, and gain knowledge about reading promotion.

The leader talked about youth volunteer training in CL7.

\begin{abstract}
There have been 3 to 4 generations of youth who work here. The first generation is in their third and fourth year at universities. Sometimes, after they finished their high school and furthered their study in the cities, we didn't have any reading promoters left. Right now I am training younger children to be reading volunteers. They will be trained from primary school to open the $\mathrm{CL}$, clean the $\mathrm{CL}$, arrange the shelves, and read picture books to young children. I normally train older children about reading activities for children, for example, picture book reading techniques, and the activities planning process. Then they will plan how to approach each village... (CL7 Pradit).
\end{abstract}

The ECT mentioned that all groups of people in the community benefit from CL7, especially young children up to age 12 , as mentioned in 9.3.5. All of her three sons had volunteered to read to young children. The oldest is 22 now and he also brought his friends to do reading activities. In addition, she noticed that the teenagers who were reading volunteers are generally nice, keen on studying, and they show leadership. Boonta, the former volunteer, recalled her experiences and benefits from being a volunteer in CL7, where she was persuaded to be a library volunteer when she was 13 . She thought the $\mathrm{CL}$ benefits the reading volunteer or reading leaders the most. She gained more confidence working with the $\mathrm{CL}$ and cannot understand why her friends at school are so shy in front of the class. However, most people cannot see the benefit of being reading leaders, thus it is not easy to find a new generation. Her talk confirms the conflict between the $\mathrm{CL}$ and a group of school teachers mentioned by Pradit in 9.3.1.1. In CL8 the former youth volunteer also shared his experience in the urban context when he was 18. TV footage showed that youth empowerment was very strong in the CL. Youth groups had actively organised many fundraising and other activities to establish the CL. Participants mentioned in 9.3.2.1 that people who work in the library must be kind and friendly. They should be readers and have some knowledge of age appropriate books, especially for young children. In CL8, which operates similarly to a CDC, the volunteer mentioned that the workers need to be able to care for young children, help with the children's homework, take care of books, and take care of those using the library. For 
reading leaders, CL8's training focused on storytelling principles and techniques, facilitator creativity and planning processes, leadership, and presentation skills, as mentioned previously by Pradit and Boonta. Other key characteristics of reading promoters mentioned are teamwork, tactfulness, talking and persuading skills, and soft skills needed to deal with children.

Training is an important factor mentioned by the leaders and ECT in the case. The ECT talked about the hand-made picture book training sessions organised by Happy Reading which opened her to the world to arts and reading promotion. She expressed positive outcomes in terms of confidence and the capability to lead reading activities and teach others. The training brought self-discovery that transformed her from an unconfident ECT to be a trainer. The training really ignited her creativity and confidence. She was more confident in finding that the young children like these inexpensive picture books.

Teachers who had participated in other capability building training provided by $\mathrm{CL} 7$ also reflected that the training sessions enhanced their creative skills and the process of reading, thinking, analysing, and presenting work. The positive outcome was that the teachers talked and exchanged ideas more. They were hugely inspired to develop their artistic potential.

These case findings show that with appropriate training opportunities, an individual who has no background or confidence could develop themselves to be leaders in RfP.

\subsection{Chapter Summary}

In summary, participants in Case 4 did not have many opinions about policy but were more focused on the community level, emphasising that parents and sub-district organisations needed to be more involved with the CLs. Since both CLs were established before the national reading policy, the leaders stressed that the policy needs to be disseminated consistently to small towns, which appeared not to benefit from the policy in both cases.

Since CL7 received strong support from Happy Reading, the scope of CL7's work was extended from the villages to the district level and involved a variety of activities. Both CLs were exceptional regarding the youth volunteer spirit. However, while CL7 continued 
to benefit from Happy Reading support, and a new batch of young people had joined its operations, five years after CL8's inception it was difficult to find a volunteer officer to work in the library. Since Happy Reading did not fund the library, there was a need to have student volunteers those can help raise more funds for book acquisitions.

CL7 showed that even for some educated Isan people, for example Pradit and Boonta, rural Isan inferiority complex is identified as a factor negatively affecting confidence and library visits. Luckily, those participants had alternative supplies of books, including from the CLs. The case did not directly involve more disadvantaged users. However, the comments on meals provided to hungry children in $\mathrm{CL7}$, and friendly interaction with beggars in CL8 reflect that the CLs provide not only the extremely low-anxiety atmosphere that nurtures RfP in a marginalised population but also humanitarian actions, highlighting inclusion and book access issues.

Individuals in Case 4 revealed that although they did not have any background in libraries and books for young children, their grants from Happy Reading, and on-the-job training developed them into local leaders in RfP. Similarly, practical training sessions on picture book production, literacy development, and ECD were successful in fostering interest and confidence in reading and art in the youth volunteers and ECT.

Having the freedom to relax and read in the CLs, and the varied book choices provided in both CLs show that the CLs operate from a non-authoritarian, and a non- knowledgeoriented mind-set. The middle aged female library volunteer also characterised a nonnormative villager who was keen to seek education, did not feel inferior and shy, dared to volunteer, and be different from the majority. 


\section{CHAPTER 10 Factors Affecting Reading for Pleasure in Community Libraries}

\subsection{Introduction}

The case study reports 1 to 4 (Chapters 6 to 9) address the first and second research questions: what are the factors affecting RfP reading promotion practice in CLs in the Northeast region of Thailand, and how do these factors facilitate or hinder RfP reading promotion practice in the CLs? Taking a broad and holistic perspective, this chapter revisits both research questions.

By combining, comparing and contrasting the findings from each case, this chapter provides a higher level of interpretation across four key themes (see Table 10.1). The themes identify the four holistic conceptual factors derived from data in each case and show how the factors facilitate or hinder RfP reading promotion practice in CLs in Thailand, using the theoretical lenses identified in Chapters 2. As factors in the study are interwoven constructs, the chapter is structured based on the potential of each factor for telling a well-integrated story, as shown in Table 10.1, instead of following the same structure as Chapters 6 to 9.

Table 10.1 Themes and factors affecting RfP reading promotion practice in CLs

\begin{tabular}{|c|c|c|}
\hline Factors & $\begin{array}{c}\text { Elements in } \\
\text { FVR }\end{array}$ & $\begin{array}{c}\text { Elements in Ecological } \\
\text { Framework }\end{array}$ \\
\hline \multicolumn{3}{|c|}{ Theme A: Provision of Book Access in Disadvantaged Areas } \\
\hline $\begin{array}{l}\text { reading materials, national reading } \\
\text { policy, coordination between } \\
\text { supporting organisations, book } \\
\text { market and book purchase }\end{array}$ & $\begin{array}{l}\text { - book access } \\
\text { - great variety of } \\
\text { books/choice of reading } \\
\text { material } \\
\text { - light reading materials } \\
\text { - minimum censorship }\end{array}$ & $\begin{array}{l}\text { - collection to suit the needs of all } \\
\text { people } \\
\text { - interaction with community } \\
\text { members to respond to community } \\
\text { needs with materials and program }\end{array}$ \\
\hline
\end{tabular}


Table 10.1 Themes and factors affecting RfP reading promotion practice in CLs (cont.)

\begin{tabular}{|c|c|c|}
\hline \multicolumn{3}{|c|}{ Theme B: Literacy Development Support } \\
\hline $\begin{array}{l}\text { literacy traditions, normative } \\
\text { culture, feminine society and } \\
\text { religions, attitudes towards RfP, } \\
\text { parents' migration and parenting, } \\
\text { media, picture books and reading } \\
\text { to young children, literacy } \\
\text { problems and MOE literacy policy, } \\
\text { school libraries, and reading } \\
\text { activities }\end{array}$ & $\begin{array}{l}\text { - low-anxiety situation } \\
\text { - light reading materials } \\
\text { - effortless reading } \\
\text { - minimum censorship } \\
\text { - reading role model } \\
\text { - storytimes } \\
\text { - rewards }\end{array}$ & $\begin{array}{l}\text { - attempts to meet the needs } \\
\text { and interests of community } \\
\text { members } \\
\text { - interaction with community } \\
\text { members to respond to } \\
\text { community needs with } \\
\text { materials and program } \\
\text { - library staff are capable of } \\
\text { building community } \\
\text { connections }\end{array}$ \\
\hline \multicolumn{3}{|c|}{ Theme C: Provision of an Inclusive, Safe Atmosphere } \\
\hline $\begin{array}{l}\text { learning atmosphere and location, } \\
\text { public libraries and librarians, } \\
\text { education, Isan identity and Isan } \\
\text { language, and community } \\
\text { participation }\end{array}$ & $\begin{array}{l}\text { - low-anxiety situation } \\
\text { - library access of } \\
\text { children from low SES } \\
\text { - omission of } \\
\text { accountability } \\
\text { - role of public library }\end{array}$ & $\begin{array}{l}\text {-library staff change their book } \\
\text { guardians image to that of } \\
\text { learners, leaders, promoters } \\
\text {-CL operates in welcoming } \\
\text { ways and is inclusive for } \\
\text { diverse gender, cultural, and } \\
\text { linguistic needs } \\
\text { - library staff are capable of } \\
\text { building community } \\
\text { connections } \\
\text { - physical location as a center } \\
\text { of community } \\
\text { - CL provides safety, and is } \\
\text { accessible to schools and } \\
\text { community services }\end{array}$ \\
\hline \multicolumn{3}{|c|}{ Theme D: Active Roles of Key Individuals in RfP } \\
\hline $\begin{array}{l}\text { leaders in RfP, reading models and } \\
\text { training for Bookstart Projects, } \\
\text { family members, teachers, reading } \\
\text { volunteers/reading leaders, and } \\
\text { other individuals }\end{array}$ & $\begin{array}{l}\text { - book access } \\
\text { - library access of } \\
\text { children from low SES } \\
\text { - low-anxiety situation }\end{array}$ & $\begin{array}{l}\text { - training workshops for } \\
\text { librarians } \\
\text { - library staff forge meaningful } \\
\text { partnerships and work with } \\
\text { their community stakeholders }\end{array}$ \\
\hline
\end{tabular}




\subsection{Theme A: Provision of Book Access in Disadvantaged Areas}

The findings show that the provision of book access in disadvantaged areas is an important way for the CLs to promote RfP. The findings from all cases indicated that family members and their attitudes towards child-rearing are also influential factors for encouraging RfP. Theme A involves reading materials and most of the broad Thai reading contextual factors: the national reading policy; coordination between supporting organisations; and the book market and book purchase. It is worth noting that picture books are the most important reading materials identified in most cases; however, as it is more relevant to Theme $B$, it is presented in 10.3.6.

\subsubsection{Reading Materials}

Findings reveal that all the CLs in this study play strong roles in providing a choice of reading materials for people in deprived areas, particularly children. In all, much of the evidence indicates that children, adults, and older people in rural areas do read but generally they do not have quality materials with enough variety to suit their interests. The assertion that villagers have an appetite for books and are eager to read beyond the availability of the limited books provided by the CLs was confirmed by leaders in RfP. These statements conform with Krashen's assertion that people will read if libraries supply books which meet their reading needs (Krashen, 2011a).

'Reading materials' is an important factor identified in all cases because the primary function of most CLs is to provide book access, particularly books for children, which are scarce in rural areas. However, in Case 2 the ONIE SBHs were severely under-resourced and therefore providing a range of quality reading materials is a challenge.

\subsubsection{Responsiveness to Local Needs}

The Ecological Framework identifies that responsiveness to local needs is a crucial aspect in $\mathrm{CL}$ management. In most cases books and serials are selected for the CLs after considering the community context and the reading preferences of local people. In CL1, borrowing records show that non-fiction, for example Korean, Japanese, and Thai educational comics for children, are very popular. The large number of 400 books lost 
from the library reflects that people and CDCs in the area want picture books, fiction, and educational comics for children.

Case 2 shows this clearly since the DPL realized that the provision of low-quality reading materials that are not suited for the villagers reading interests is the most critical issue negatively affecting RfP in ONIE CLs. This point is strongly confirmed by other participants in the case and they agreed that newspaper and magazine provision during 2014-2015 in SBHs greatly encouraged villagers to read for pleasure. Therefore, the subscription cuts severely affected RfP in rural Thailand. To be specific, there were fewer users visiting the CLs, and people felt frustrated about the lack of availability of serials. Participants shared their concerns that without additional reading material, RfP practices will largely cease and the CLs will not be sustained. The issue of sustainability is confirmed in the CL7 area where, without serial supply, the SBH in the village was closed due to the lack of additional reading materials.

Case 2 shows that government investment in the provision of daily newspapers is beneficial because they are the most popular reading material for rural people. In addition, magazines, for example, Koo Sang Koo Som, and Kwan Reun, are identified as popular among female villagers who read them for general knowledge and crafts. In other cases, cookbooks, books on local herbs, Buddha's teaching, gossip magazines, agriculture, and the health column in newspapers were also mentioned.

\subsubsection{Variety, and Quality}

In all cases, the provision of selected children books and activities related to reading to young children shows that book variety fundamentally supports RfP. The study reveals that the prominent leaders in RfP, for example the WA expert (CL1) and Naree (CL6), realised that variety is crucial. However, Naree is the only participant who emphasised minimum censorship in book selection. This is due to her work as a health promotor where she could see that book of one genre, for example, romances, may initially support RfP, but do not support the mental growth that is important for progressing to read more demanding materials. Therefore, she stressed that children need to be exposed to a great variety of reading materials, and she put minimum censorship on her Book Bank project. 
Every case confirms the importance of the variety and quality of books. However, none of the CLs had a stable supply of new books, except in CL5 that published picture books. Most CLs have their most active period when they acquire new books; for example, CL1 had a large collection from an international charity that cost around 100,000 baht in 2007 that led to an increase in use at that time. CL8 is so successful in terms of youth empowerment and fundraising activities for collection development that TV programmes broadcast their success nationwide. However, after a few years when the leaders in RfP turned their interest away from most CLs, and the CLs had not been supplied with new acquisitions, the CL's operation greatly deteriorated.

The interviews with the DPL, former ONIE executive, and SOTs in Case 2 portray an inadequate variety, quality, and number of books in ONIE CLs which had an adverse effect on RfP in this largest CL initiative in the country. The DPL questioned the quality of reading materials purchased by the district ONIE. She had to select books from those supplied by ONIE before distributing them to the villagers because she received complaints from the villagers who found the books dated and uninteresting.

\subsubsection{Book Donation}

While book donation in urban areas, for example the Book Bank in CL6, tends to receive books with sufficient quality, the Book Voyage, an ONIE book donation project in rural areas, is identified as an ineffective way to acquire quality books for the CLs. The books that the villagers donated to the DPL in Case 2 were of low quality which generated excessive delivery and weeding work. Similarly, CL7's campaign for book donations also found that most of the donated books were old textbooks.

Therefore, in all cases which focus on nurturing the love of reading and literacy development in young children, book donations were not accepted. Picture books found in the study are newly bought or published by the CLs. Although Naree (CL6) supported book donation and book exchange for adults, she used donation money received from the Book Bank to buy children's books for rotation in reading areas in the Book Bank spot and for book acquisition for CL6.1.

Similarly, after multiple discussions on how to acquire quality reading materials for projects in the district, CL7 used their funds from Happy Reading to provide a budget for 
each CDC to buy children's books. A school teacher involved with CL7 mentioned a new approach to acquiring new books for school libraries by encouraging parents to buy books to offer to the monk, instead of money, as a form of merit making.

\subsubsection{Light Reading Materials and Book Addiction}

With regards to light reading materials, most participants have a positive attitude towards RfP and light reading, seeing it as a conduit to more demanding reading. In Case 1, participants mentioned that reading light material, for example, fiction and comics, actually requires more brain function, concentration, and imagination than non-fiction. These materials also expose children to various experiences and a great variety of vocabulary. In CL1, CL5 and CL8 most of the popular picture books, comic series, and novels disappeared from the CLs, showing that people love to read light materials.

However, in all cases, participants often mention Thai parents' knowledge-oriented mind-set and their authoritarianism which may result in control over light reading materials for children. In Case 3, Naree observed that Thai parents are likely to be reluctant to support light reading, and their mind-set is applied to children but not to the adults themselves who are also tempted to read comics. Boonta in Case 4 portrayed the norm for parents from rural areas and their lack of knowledge of RfP. This is in line with Krashen's view that control over book choice hinders RfP. Parental control did not seem to work against book addiction in several cases mentioned, however; for example, Boonta read novels despite her parents' prohibition. However, in a less rebellious case, Naree mentioned an obedient girl whose mother prohibited comic reading, and her chance to practice RfP was removed. Thus, a better understanding of RfP in adults is more crucial than in children.

The WA expert in CL1 mentioned that books that suit a variety of reading abilities will attract readers, therefore, variety is crucial. He also gave the examples of both light and hard materials that suit different reading abilities and reading interests. The most distinct example of children with very high reading ability and interest is an 8-year old reader-Naree, in the WA project who read the lengthy Five Hundred Lives of the Lord Buddha. Naree's literacy development has been nurtured by a variety of easy, lowquality one-baht Thai comics that her mother bought for her every day when she was 
young. This example supports Krashen and Ujiie's (2005) assertion that junk/entertaining reading is good for children.

These book addiction feelings which the FRV concept describes as a state in which readers are "hooked" and "lose" themselves in the book, were mentioned in all cases both in terms of personal reading experiences, and observers' experiences. This allies with an FVR principle of providing a variety of reading materials and limiting control over book choice.

\subsubsection{National Reading Policy}

The Decade of Reading Agenda 2009-2018 was commented on at both the broader and the case-specific levels. At the national level, participants in all cases except Case 2 (ONIE's SBHs) found that it had no impact on book access in rural areas, urban poor areas, and small towns in which the CLs are located. Leaders those are in favour of a RfP awareness raising campaign targeting both the general public and teachers are mostly identified as non-readers in Cases 1 and 3. In Case 3, leaders stressed the importance of raising awareness and the establishment of a reading culture that begins from nurturing RfP from a young age. Naree's interview in 8.2.2 indicates that this PhD study would articulate this awareness because, as of 2016 , government policy had not recognized RfP. Yada in 8.3.1.3 also asserted that reading to young children is never mentioned by Thai academics.

The WA expert (CL1), and Yada (CL5) contended that the government needs to find a process to identify and create reading leaders/reading volunteers who can encourage new readers. Yada stressed they must be persons "who will not let go of things easily and those who are not waiting for the policy from their boss. Because if we wait for the policy, it will never happen". As a government officer, she argued that progress should start at the local level without waiting for government policies, which she found unstable. The case shows that to promote reading culture in the country, civic involvement and leadership are crucial.

The WA expert made strong comments on event-based reading promotion, suggesting that it occasionally brings a festive feeling to the public but has not yielded any real change. The interviewed leaders suggested the government to maintain policy stability 
and provide reading promotors with adequate and practical details, practice, and reading materials for each project. In Case 3, the urban poor community leader commented that an Early Childhood Literacy Fund (ECLF) should be made an explicit policy nationwide because the current complicated process has resulted in difficulties for her marginalised urban poor community in reaching the policy. Yada added that the agenda would be sustained if there are more parties involved to form a network, which is shown in CL6 and CL6.1 that involved Happy Reading and other organisations in the area.

At the case-specific level, Happy Reading, which was established under the Decade of Reading Agenda in 2010, greatly contributed to the success of CL6 and CL7. Since Happy Reading aims to promote healthy living and strengthen civil society, activities in the CLs vigorously reflected these purposes. The large grants these independent CLs continuously received from Happy Reading since 2010 made their activities outstanding in terms of variety and impacts at the district and provincial levels. Their other strongest point as a result of this national support is the continuous training provided by Happy Reading. Therefore, they were actively cooperating with numerous projects in 2015.

\subsubsection{Coordination between Supporting Organisations}

The study reveals that coordination between supporting organisations is crucial for book access in disadvantaged areas which is considered as expert knowledge to raise RfP awareness. In Cases 1 and 3, an international charity generously provided a large budget to the WA to acquire a large collection and provide expert knowledge to CL1 and CL4. The expertise of the retired ONIE executive was crucial to coordinate and acquire quality books for CL4. The richness of the collection encouraged RfP in both areas, where the CLs also served primary schools and CDCs. While CL1 has had no additional books since its establishment, CL4 has an extensive collection that has been contributed to by other organisations. Therefore, it could extend its operations to branches in other villages.

Case 2 shows a close connection between ONIE SBHs and public libraries, and the ambiguous connection between the DPL and SOTs which negatively affected the CLs' operations. Findings show that the success of an ONIE CL is largely based on a DPL who voluntarily worked beyond her job description. 
The most advanced and autonomous case is CL5 that have a PHC director who is able to strategically combine her Master's degree research, academic connections, and the TAO to support her BSP. The CL gained several copyrights from a renowned picture book author, thus the director can publish picture books locally at very cheap prices to use in the BSP. The BSP has increased book ownership in families that generally have no reading materials. In another example of successful coordination, the strong support given by Happy Reading to CL6 and CL8 resulted in a great variety and broad impact of the activities presented in Cases 3 and 4.

The TAO and ONIE, as mentioned by participants, stated that if they could find a way to cooperate they can enhance RfP. However, in Case 2 the degree of cooperation tends to depend on individual SOTs. In CL1, the drastic contrast between the initial start with full expert knowledge and support given by the WA and a year later when it was transferred from the temple to the TAO shows the TAO's lack of knowledge of library operations. The librarian tried to submit new book lists to the TAO but the new purchases were denied. Thus, after 9 years with half of the books lost from the library, library visits greatly declined. In late 2016, CL1 was closed due to a local political conflict, reflecting that a $\mathrm{CL}$ under a TAO is vulnerable to both financial problems and local political instability.

\subsubsection{Book Market and Book Purchase}

The 'book market' is a complex factor entwined with socioeconomic status and attitudes toward RfP. The WA expert contended that the government needs to focus on a practical way to address problems within the book distribution system. The fact that book prices in Thailand are generally high, as shown in figures in section 3.2.3 and A.4 (Chapter 11) which usually prohibits rural people who generally have only a seasonal income, from buying books. However, in Cases 3 and 4, the leaders mentioned that the villagers who have money and those in the middle class will buy books to read for pleasure if they understand and see the benefits of RfP for literacy development.

Giving rural people the chance to access quality reading materials is crucial. The former ONIE executive stressed the connection between the opportunity to access books and RfP, indicating that reading in Thailand is not only about class but also the access to 
quality reading materials. While middle-class people have greater access through many means, rural people can often only access dated donated books in ONIE CLs.

In conclusion, there are three factors related to CLs' roles in the provision of book access in disadvantaged areas. First, book prices disadvantage poor people who might not have the money to buy books. Second, people need to value RfP. The data suggest that book purchasing behaviour depends on attitudes towards RfP. Pradit's comments in 9.3.4 show the connection between training sessions offered by CL7, awareness amongst parents of the value of RfP, book purchasing, and better literacy development. Third, the shortage of books and bookstores in rural areas contributes to the inaccessibility of books. The consignment system and low demand for children's books is explained by an expert from CL1 in 6.2.5.

\subsection{Theme B: Literacy Development Support}

Theme B presents factors affecting RfP in CLs in relation to literacy development support, which the study finds to be a major potential role of CLs. The study shows that in all cases, literacy traditions, parents' migration, and current parenting practices negatively affect literacy development in children during a period of rapid brain development (0-6 years). Therefore, CLs play a key role in filling the gap that contributes to the country's longstanding literacy problems.

Relevant factors in the theme include: 1) literacy traditions; 2) normative culture, feminine society, and religions; 3) attitudes towards RfP; 4) parents' migration and parenting; 5) media; 6) picture books and reading to young children; 7) literacy problems and the MOE literacy policy; 8) school libraries; and 9) reading activities.

\subsubsection{Literacy Traditions}

The study finds that literacy traditions fundamentally affect how parents and teachers support literacy development in young children, including whether they supply books for older children to read for pleasure. Most participants' comments on the first tradition, the teacher-based learning style, reflect that this approach is still prevalent in rural areas. Findings in all cases reveal that parents in rural areas tend not involve 
themselves with reading to young children because they believe that children are too young to understand.

The next tradition, the firm knowledge-oriented mind-set, encourages most villagers and early childhood teachers in the villages to buy Thai $A B C$ books, and force young children aged 2 to 3 to remember and write the letters of the alphabet. Leaders in most cases argued that this belief and practice is against modern ECD principles. The leaders found that this kind of demand from authority figures might result in children avoiding reading and learning in general.

The parents' third norm is a lack of knowledge of RfP due to the absence of books and book-related activity in rural areas. This is a contextual factor added to the knowledgeoriented mind-set. In Case 4, Boonta shared that her parents hid novels from her and supported her to buy only textbooks, exemplifying the norm in parents who lack knowledge of RfP.

Although participants identified that there has been a decline in the prevalence of teacher-based learning because of technological advancements, leaders in most cases stressed that reading promoters need to spend a lot of energy on promoting reading to young children and RfP. Roughly half of the participants felt that Thai teachers and educators are open to RfP while the other half stated that teachers still hold the belief that books should contain only knowledge.

In terms of oral tradition, this is mentioned as a positive factor affecting RfP in Cases 3 and 4 . In some cases, bedtime stories told by caregivers and oral storytelling are identified as creative mechanisms to enhance RfP. A folklore project developed in a school library by the CL5 leader not only encouraged writing and reading skills in elementary school students but also enhanced bonding between the elderly and children in the family. The Thai language teacher who led the project proudly talked about the handmade books and the school library award received for the activity based on the project. The case allies with the Ecological Framework, showing a seamless project engaging CL5, the teachers, the school libraries, and the communities. In this way, reading and the $\mathrm{CL}$ play a vital role in the community. 
The influence of the oral tradition and talking was asserted by two leaders in RfP in CL5 and CL6 who mentioned that music, storytelling, and poetic dialogues in traditional plays, are RfP because of their link to imagination. The oral literature projects in CL5, CL6, and CL7 also support the building of social capital and family ties as well as the preservation of the oral tradition. However, in Case 4, the leader noted a decline in the use of folklore and the need for its preservation due to its role in helping maintain Isan heritage.

\subsubsection{Normative Culture, Feminine Society, and Religions}

Findings show that a normative culture and feminine society tend to obstruct reading to young children and voluntary work in CLs. However, religions greatly support these activities. All CLs mentioned that due to the normative culture, it is difficult to advocate for new ideas, particularly reading to young children.

Moreover, the distinct extreme feminine character of the Thai society which does not support individuals acting differently from the majority, makes any pioneers of new ideas and voluntary work feel uncomfortable. Case 3, CL7 portray a situation in which involvement in library volunteer work, which greatly supports rural children's literacy development, was seen by others and even teachers as an unusual and undesirable activity.

In terms of religions, both Buddhism and Christianity contribute to RfP in CLs in Isan. CL1 is in a Buddhist temple compound and the Abbot is a leader in RfP who leads the community and seeks academic help from the WA to establish the CL. The DPL in CL4 also mentioned that her public library was formerly located in a Buddhist temple and the latest abbot greatly supported the library.

The Buddhist belief in merit-making in the form of social work has a large impact on RfP in CLs because they depend primarily on volunteers. A leader in CL8 commented that in Thailand it is hard to find people with a volunteer spirit. She saw that people still believe that merit can be gained only through traditional rituals in Buddhist temples. Findings showed that in most cases, library volunteers were middle age women who selfidentified as uncommon/strange villager, and youths as mentioned in 10.3.7. Dara, a library volunteer in CL5, disagreed with the majority who thought that merit making 
could be done only in the traditional ways. She knew that some villagers did not like her outstanding voluntary work but she did not care, which is different for Boonta in CL7 who cared about moral support from adults and teachers.

Another aspect of Buddhism is the spiritual crafts learned from books and training provided by CLs which brought great pleasure to the female villagers in Case 2. It also provided additional income for the villages if others bought their work.

There were three CLs positively affected by Christianity. The urban Christian charity school in CL2 provided educational support for two generations, the CL officer, and her children. The school also provided a large collection of children's books in the school library, which supports literacy development. CL5 and CL6 were located in two different provinces in Isan but revealed the influence of modern education in three generations which positively affected their views and practice of RfP. In CL5, the reading model who regularly read to her grandchildren attended the Christian private school in the village, and started to be a reader in an urban Christian school. After she left the school she continued reading romances that her school friends brought to her from the city.

In CL6, the leaders' grandfather, who loved to read, became a Protestant a hundred years ago. He sent their mother to a Missionary School which could be considered progressive for the time. CL6 showed the influence of that education through the liberal way in which both were raised, and their reading and social work. The upbringing resulted in two outstanding leaders in RfP in Isan who had been trainers for many Happy Reading sessions. Naree was possibly the most advanced leader in RfP in the country because years before this research she clearly identified that literacy traditions are the biggest challenge for reading promotion in her first funding proposal submitted to Happy Reading. The grant facilitated her to run BSP and the Book Bank Project.

\subsubsection{Attitudes towards RfP}

All cases show that all participants have positive attitudes towards, and a good understanding of, RfP. Most participants who are readers identified their "forgetting" or "lost in the book" experiences, which is allied with Krashen's FVR principles focusing on the affective aspects of RfP. Most agreed that despite low SES, positive attitudes towards RfP lead to voluntary reading behaviour and better literacy development. The 
ECT in CL7 and Naree in CL6, affirmed that with strong positive attitudes towards RfP, their poor background and limited exposure to appropriate reading materials could not obstruct their desire to be readers.

In all cases, the findings show that it is challenging to change the attitude of Thai parents toward RfP. Case 3 provides an explanation for this. That is, "reading" and "pleasure" are two contradictory concepts in Thai understanding. On the one hand, the knowledgeoriented mind-set toward reading and learning, as discussed in 8.3.1.2, makes most Thai people believe that reading is only for academic purposes. On the other hand, the word "pleasure" connotes only enjoyment without academic benefit (see section 8.3.4). This greatly affects the take-up and understanding of RfP.

However, Case 2 shows that in the rural Isan context, RfP might be connected with both leisure and additional income as presented in 10.3.9.3. Affective Aspects of Reading Activities in CLs. The explanation by the former ONIE executive in 7.3.4 reflects a combination of the strong Thai knowledge-oriented mind-set and the Western RfP concept.

\subsubsection{Parents' Migration and Parenting}

Parents' migration is mentioned concurrently with poverty and parenting in Isan. In all cases, participants described at length about the negative impact of parents' migration on the 'left-behind' children's literacy development. They remarked on financial constraints and the limited parenting capacity of elderly people. However, a case in CL6 showed the success of a migrated couple who had the ability to find good jobs and fully support their children's literacy development through Nida, the grandmother. The girls were successful at school. Similarly, as observed by Naree some grandparents can provide good care for the children.

In most cases, participants noted that most grandparents maintain conservative views on books and reading and mostly cannot afford to buy books for the home. Participants were uncertain whether those grandparents who were even provided with books in the BSP package read them to the children. Not only do they have eyesight problems and have limited literacy skills themselves but they also think that there is no need to read because young children could not understand. Moreover, the DPL found that the elderly 
did not allow their grandchildren to visit the $\mathrm{CL}$, which can cause a double negative impact on their opportunity to access books.

Another indirect factor hindering RfP caused by parents' migration is excessive materialism, for example smartphones and tablets that migrant parents bought for their children. Nida's story in section 8.3.5 about her daughter, who wanted to provide a tablet PC for her young daughters shows the power of RfP over an IT device. The teacher in CL5 observed the same good intentions - that some migrated parents give their children IT devices but they do not have the ECD knowledge and time to train their children to use them. Therefore, information literacy and digital literacy were mentioned as a necessity in the primary school curriculum. Moreover, participants worried about the elderly leaving grandchildren with a smartphone because the older people believe that children who can use smartphones are clever, so they do not read books to them.

\subsubsection{Media}

There is a paradox of openness in Thai parenting that rural people are fully open to mobile and internet technologies. This is contradictory to the firm literacy traditions as discussed in 10.3.2. Most participants in Cases 1, 2, and 3 expressed their great concern about the excessive use of screen technology in Thai society and concern was particularly expressed about the elderly and parents who had limited understanding of the impact of screen technology on literacy development in young children.

The findings signify that parenting skills, knowledge about ECD, and digital literacy skills are factors related to media exposure that can affect RfP. In most cases, findings show that participants could not avoid this disruptive technology. Participants cannot see any solution to the impact of this tremendous technology advancement on RfP. Only in Case 3 did the nursery owner suggest that adults need to talk to children about limiting their exposure to media. In most cases, participants identified that media addiction in children and teenagers greatly affected CL visits in Isan.

A middle-class father in Case 1 mentioned that high book prices in the country make IT gadgets seem a better investment in terms of reading. However, he believed that modern media cannot enhance children's literacy development as much as reading from physical books. 
Social media was used positively to establish CL8, and to invite people to join in activities in CL6 and CL6.1. Digital reading was mentioned in CL2 and CL8. Participants suggested that reading promoters need to think creatively about new ways to promote RfP via social media and smartphones.

Other media the participants saw commonly used with children were TVs and story CDs in village CDCs and ONIE's mobile library. In Case 3, as observed by the nursery owner in CL6, ECD teachers and primary school teachers frequently use these media but rarely read to children. Leaders in Case 3 firmly agreed that the mobile library should not have these devices because they distract young children from RfP.

\subsubsection{Picture Books and Reading to Young Children}

The study not only finds that participants in all cases are convinced that picture books fundamentally support literacy development, but also that disadvantaged children and people are attracted to the beauty and quality of these books. The observation and archival photos also show rural children's delighted faces when they touched picture books, and the unconscious "lost in the story" faces when they were read by the library officers or caregivers.

The evidence in Nida's case (CL5) shows that the affective aspects (delight, safe, warm feelings), fascination, and function of picture books as literacy development tools are allied with Krashen's FVR principle on understandable text in low anxiety situations. The affective aspect of storytimes also allies with Krashen's second language acquisition theory outlined in 2.3.3.

An expert in Case 1 found that the gap in the opportunity for middle class and poor children to be read to was huge. This assertion was supported by a middle-class father in Case 1 and other participants in all cases identifying the rarity of picture books in rural areas and the reaction of rural villagers to storytimes, which is a reflection of the previously mentioned literacy traditions. The pioneer who read to children alone in Case 1 was gossiped about by the people in urban poor area. In Case 3, however, Yada said that CL5 helped mothers who had recently given birth to find some friends to read to their babies together; thus, they do not feel strange. These interviews confirmed that 
many people in Thai society are unaware of the connection between listening, reading, and writing and therefore highlight the importance of training and awareness raising.

Time allocated to reading engagement was uncommon in Isan families according to participants who noted that, in both urban and rural areas, Thai caregivers tend to prioritise their work over parenting. Thus, there is less time to communicate with their children, particularly when they are young and need brain stimulation through communication. However, Nida's engagement with her grandchildren showed that reading to young children is easy. Also, she by chance did what the WA in Case 1 found to be effective: 1) caregivers need to read regularly and consistently to children, and 2) caregivers need to read a good range of books. Moreover, the essential factors that make her case a very successful reading model were that she was a keen reader who cares about every aspect of parenting. She showed that with substantial financial support from migrated parents and a grandmother's care, a BSP can contribute to noticeable literacy development and healthy living in rural communities. In addition, an observation in CL6 conformed to the WA's conditions. The storytimes performed by Nalinee twice a day with a good range of books from CL6 tremendously helped children who had problems caused by excessive exposure to TVs and smartphones, and/or who had behaviour problems.

Although most leaders felt that it was challenging to change parents'/caregivers' beliefs about literacy development, they reflected that the community are open to changes based on the three conditions presented in 10.5.1 Reading Models. Nowadays, after experiencing the positive outcomes of BSP by seeing reading models, parents are more likely to understand that they need to engage in literacy development and gradually change their attitudes on home-school partnerships.

The degree to which parents read to young children varies from case to case, with the highest estimation in Case 3 (60\% in CL5), and the lowest in Case 2 (10\% in CL3, and 25\% in CL4). This may occur because the health promoters in Case 3 had a number of active VHVs able to print and supply free picture books to parents. In contrast, in Case 2, CL3 did not receive any picture books either from ONIE or any other external body. Thus, the active SOT and the DPL started to introduce picture books to the villagers in late 2015, 
which was much later than CL4 and most CLs studied. Many ONIE SOTs ignored the ONIE additional early literacy development tasks due to their multiple responsibilities for adult education, and having no suitable book supply in the CLs. Moreover, the ambiguous role of the DPL, and the fact that picture books need to be bought and distributed from a public library also contributed to the SOT's ineffective support for early literacy policy, as mentioned by the DPL throughout case report 2 .

\subsubsection{Literacy Problems and MOE Literacy Policy}

All cases show that a resolution to literacy problems is crucial for Isan, revolving around literacy development, reading skills, literacy traditions, and book access. Leaders in Case 1 identified that book access at the suitable reading level is a major factor bringing reading enjoyment. The exposure to picture books and lively storytimes, and a strategy by older school library volunteer students, help alleviate literacy problems.

The MOE's literacy policy was a critical contextual factor during 2014-2015. It was commented on in all cases as too demanding for both the first graders and teachers. Leaders in RfP and teachers mentioned that this policy needs more practical guidelines, interesting reading materials, and teacher involvement. Most leaders commented that the focus on reading skills and the traditional knowledge-oriented mind-set might too forceful and this actually hindered children's ability to read at a young age. It may also have affected attitude toward reading. The leader in Case 2, added that MOE reading materials are not interesting and too challenging for the young learners, which may create a high-anxiety situation, which is against FVR principles (Krashen, 2011a).

A primary school teacher in Case 3 commented on the change from the traditional phonics approach to a whole language approach that significantly affects how teachers manage their CT language teaching. She also added that the MOE needs to understand that literacy development in rural areas takes more time than in urban areas due to the nature of the work of rural parents. Her comments link with Krashen's point that FVR is not for early learners, who generally need a phonics approach.

Case 2 shows that this top-down, ill- prepared MOE literacy policy that SBHs were instructed to apply and evaluate less than a year after its introduction resulted in conflict between the DPL and inactive SOTs. The DPL found that there were only a few SOTs 
actively implementing the policy. However, she was required to record percentages and provide photographic evidence of children attending library activities from November 2015 to March 2016 and report on how many were literate before age 6 to the Central ONIE. She took action by using the public library acquisition budget to buy more books for young children and work with some active SOTs and village reading volunteers. However, as the district has more than 50 villages, she had to estimate many figures.

\subsubsection{School Libraries}

The findings show that school libraries have a close connection with CLs. In all cases, CLs serve school students, and the leaders in each CL engaged teachers and students in CLS initiatives, particularly teachers in early childhood and elementary schools. Therefore, teachers and teacher-librarians were identified as important partners in most CLs, which reflects the Ecological Framework. Naree (CL6) identified the teacher-librarian as an important person who linked students to the library, and introduce them to the freedom to find interesting reading materials, which can ignite RfP. The fact that there is no professional teacher-librarian in primary schools greatly that hinders library operations and appropriate literacy development support which could lead to a life-long habit of RfP.

Most participants stressed that school libraries are vital for developing both RfP and reading skills. However, they are generally under-resourced due to budget constraints and the knowledge-oriented mind-set of the teachers, thus highlighting the CLs' crucial role in supporting RfP for children in rural areas.

The Abbot, a scholar who established CL1, identified that inequality of access to highquality schools in rural areas means rural children are unable to access good books at the prime time of their brain development. Most participants agreed that since school libraries in rural areas normally do not have children's books, rural children need to rely on their CLs. This highlights the CLs' crucial role in providing a suitable and great variety of materials to support literacy development and RfP for children in rural areas. 


\subsubsection{Reading Activities}

'Reading activities' is a factor that the prominent leaders considered as crucial to support literacy development in CLs. The WA expert stressed that besides making reading material available in CLs, providing adults in communities with a basic understanding of how to undertake reading activities with children should be another priority.

In general, the CLs in the study offer circulation services. However, in CL6 and CL7 that received large budgets from Happy Reading, there was also a good variety of activities organised by the NGOs, the health promotors who established the CLs. The activities were organised on a large scale ranged from public communication campaigns, youth camps, a Book Worm family camp, Reading and Riding Festivals, radio programmes, and parenting courses. They also created empowering network mechanisms and social management to support a creative media space for children and youth.

\subsubsection{Control over Book Choice and Authoritarianism}

Control over book choice is regarded by both Krashen, and Asselin and Doiron (2013) as a crucial factor affecting RfP. It is mentioned as an essential element in RfP in all cases. However, in the Thai context participants referred to the authoritarian way that adults normally control children, and choose reading material for them, as noted in 10.2.1.4 and 10.3.1.

Leaders in RfP in all cases asserted that having less authoritarianism amongst parents, friendly librarians, and giving children the freedom to choose what they read leads to RfP. The study showed that adult participants in the study generally gave freedom to children and tried to lower the power distance in their relationships. The Abbot (CL1) identified that the library should be inviting, child-oriented place and provide choices and freedom. He and the leaders in CL4 asserted that forcing children to do or read anything they do not enjoy will constrain them and lead to non-reading habits. The municipality officer (CL2) found from his experience that the more boys are forced by adults to read, the less they read. He also noted that being a role model for reading is more effective. The project report in Case 1 also portrays the relationship between RfP and storytimes that helped narrow the power distance gap between teachers and 
students, and the school principal and early childhood teachers. This makes reading an enjoyable element in the school experience for everybody.

\subsubsection{Circulation Service}

The findings show that a majority of the CLs have informal circulation services. Only CL1, which have a library officer and a formal system with borrowing cards. In CL5, regular users can easily access CLs or VHVs houses and exchange picture books without formal processes. Therefore, open circulation services and reading services in BSPs occurred at these sites. In other cases, books can be borrowed by parents and children, for example in CL4 where there is an extensive collection of books. There is a self-circulation registration notebook in which the villagers note the books they borrow. When they return books to the library, the shopkeeper sign.

Since there are few books in CL3, the DPL initiated a clippings service from newspapers subscribed to by the SBH. Thus villagers can keep, circulate, and read articles of interest. The DPL talked about this leisure activity which brought both pleasure and advancement in agriculture. The comments supported the ONIE executive's assertion in 7.3.4 that reading in the Isan context and ONIE perspective is not only for enjoyment.

Most CLs do not care if books are lost because leaders and CL officers considered that literacy development support is more important than keeping the book in the CL. Yada talked about the confessions of villagers who had taken beautiful picture books from the CL5 but she did not mind because poor children had their very first books to support RfP. She sought support from the TAO and the renowned author to print picture books and use them in her BSP, as mentioned in 8.2.5. In Case 2, Tassanee shared the same view on the nature of books lost from CLs. That is, reading materials in ONIE CLs are normally informally handed from reader to reader. A contradictory finding is that CL2 maintained a huge collection because users in the urban poor area did not borrow books. In contrast, in CL1 half of the quality books selected by WA were lost from the library. Similarly, hundreds of people who lived in the urban areas and were not in extreme poverty like the people in urban poor area, took books from CL5 and CL8 and did not return them to the CLs. 


\subsubsection{Affective Aspects of Reading Activities in CLs}

In all cases participants mentioned that sharing reading, Isan folklore storytelling, storytimes, life skill activities, and art and craft activities generated not only literacy development support but also happiness, pride, and confidence, which is an essential factor for library access as presented in 10.4.3 Public Libraries and Librarians.

Collectivism contributes to the common trait that younger children positively get the help or support of older children/adults as mentioned in CL5, CL6, and CL6.1. In most cases, older children who support the literacy development of younger ones feel proud of themselves and gain more confidence.

All cases provide evidence of shared reading, and voluntary and independent reading, as outlined by the Ecological Framework. However, if the reading happens at the CLs, the users tend to prefer to have friends to read together, as mentioned in 10.5.2. The participants mostly agreed that CL users prefer to read and talk, apart from Nida (CL5) who allocated her time for RfP by shutting the door of her house to avoid her friends. However, she also dedicated herself to leading exercises and being a reading model in CL5's training sessions.

In Case 2, RfP is seen as a leisure activity that brings both pleasure and better living, for example, an elderly woman found a method to use the abundant fruit at her home, and female villagers could gain additional income from doing crafts.

\subsubsection{The Creation of New Readers}

After long experience, the WA expert asserted that there is a greater need to create new readers than to build new libraries. Tassanee, the most experienced leader in Case 2, elaborated on the notion that the most crucial element for the creation of new readers is activities that have affective elements. Therefore, providing book access by building a library, or a movable collection is not enough. Without active officers who regularly run activities that are relevant and vital for the community, CLs cannot be sustained. Evidence for this comes from CL1, which was the last of the first generation of CLs in Isan created by the WA, but was closed down in 2016. The fact that the CL1 officer did not have any training, and therefore the CL lacked activities and could not maintain its vital 
role in the community, as suggested by the Ecological Framework. This highlights the importance of employing active officers and investing in their training.

All participants agreed that the creation of new readers is crucial. However, in disadvantaged areas, the leaders argued that it is equally vital to provide books because even after BSP training, the eagerness to read will disappear if there are no picture books to read. Yada asserted that book provision links to book exchange in the community.

\subsubsection{Reward and Accountability}

Concerning Krashen's FVR on reward, there is a variety of practice found. While in Case 1, the reward is intrinsic enjoyment derived from RfP, in CLs in more deprived areas, for example CL6.1 and CL7, the leaders not only gave praise but also provided snacks, meals and stationery to children who joined the CLs' activities. In CL8, picture books were used as awards in activities.

Most participants, who are RfP natives, viewed reading as a reward in itself so did not see the need to give any extrinsic rewards to readers. However, in CL6.1, CL7 and CL8 rewards are mentioned as bringing a fun, competitive aspect to the CLs' activities. Pradit's words on rewards, peer-influenced activities, and competitive feelings show that awards may work as trophies that young learners want to receive to be similar to their peers. Reward in his sense brings opportunity for them to see good books, and he hoped it ignited their intrinsic reading interest. Evidence from Case 3 supports his belief, because the leader in CL6 found that a reading competition at school eventually exposed her to more books, encouraged her to read more, improve her literacy, and finally experience intrinsic reading enjoyment.

Food was also provided to children by the leaders in CL6.1 and CL7; this kind of extrinsic reward is not recommended by Krashen. However, in both CLs the target group was younger than that in Krashen's work. Therefore, it may be appropriate in those cases where children are often left hungry by their caregivers. In CL7, the ECT talked about the hindrance of food because parents allowed their children to go to the library because of the free food. Thus, they did not truly understand the CL's purpose in literacy development. Therefore, she hoped that the community would get involved with the $\mathrm{CL}$ so that its aims were better understood. 
Regarding accountability, this appears to be a problem in the government system as mentioned by the DPL in 10.3.3. The estimated numbers satisfied the authorities but would not yield positive effects to the initiative. Since the CLs were not involved with book reports, only the teacher in CL5 commented on the balance between freedom, creativity, and accountability in school reading schemes. She felt that children should report on reading in creative form rather than by writing reports. This would help the student read without memorising text, thus conforming to the "forgetting" principle in FVR.

\subsection{Theme C: Provision of an Inclusive, Relaxed Atmosphere}

Theme $\mathrm{C}$ presents two social perspectives identified by FVR and the Ecological Framework. While FVR focuses on a low-anxiety situation and the freedom to select a suitable book to read without approval from an adult, the Framework focuses on inclusive policies and practices, and partnerships in the community. Since most CLs in the study were children's libraries, the findings showed that CLs have a significant role in providing relaxed, inclusive, safe spaces for the children and adults. Therefore, the theme involves: 1) learning atmosphere and location, 2) sharing reading, 3) public libraries and librarians, 4) education, poverty, Isan identity, and Isan language, and 5) community participation.

\subsubsection{Learning Atmosphere and Location}

A low-anxiety, relaxing atmosphere, and freedom in the $\mathrm{CL}$ and reading activities are identified as crucial factors affecting RfP, which allies with Krashen's FVR (2011) and Asselin and Doiron's Framework (2013). In Case 1, the WA expert, and library officers recognised that the elements of a CL's attractiveness that encourage RfP came from quality new books relevant for disadvantaged people, friendly library officers, and attractive buildings. However, he concluded that building a physical library is not as important as creating new readers. Most leaders supported this idea, but some suggested that having a physical library is preferable because displaying a variety of books in a permanent space will help support reader choice. 
In terms of location, most CLs studied are not separate buildings but local houses or community areas that are at the centre of their communities and easy to access. In Case 2, ONIE SBHs were flexible enough to relocate to new locations when the old ones had become unsuitable. In CL5, the VHVs volunteered their house to be a book hub, and they also distributed and gave book recommendations to villagers in BSPs.

Concerning the physical and psychological relaxing atmosphere, most participants agreed that CLs should be easily accessible and the users should be able to sit and read at any time. Traditional seating arrangements and the freedom to relax in $\mathrm{CL}$ spaces are crucial in most cases. In addition, in all cases, the success of CL locations is determined by the friendly library officers who are owners of or responsible for those locations, and in most cases are readers. They are considered as important persons who offer the essential relaxed atmosphere by showing that they welcome users of all SES, for example, children who wear dirty or few clothes in CL2 and beggars in CL8.

In most cases, participants who act as service providers reflected that CLs with a relaxing atmosphere encourage library visits and help create community dialogues. The atmosphere needs to be very relaxed and safe without judgment. When this is achieved, people feel they are equal and can have collective conversations on local news. In all cases, the interviews highlight the CLs' inclusiveness, and informality and approachability regarding the library access process, library workers, and library design.

\subsubsection{Sharing Reading}

All the observation and interview data reveal that sharing reading with peers at the CLS generate a literacy support process and a feeling of fun competition for children. Most importantly, by having some friends to read with, collective action makes mothers reading to their babies feel safe from the accusation of being peculiar. The positive peer influence and user freedom to select books of their choice is mentioned as enhancing for RfP in most contexts.

All cases show that collective group gatherings bring joy and created community bonding. As mentioned in 10.2.1, the publications SBHs had gained access to for only two years (2014-2015), especially newspapers and women's magazines with craft sections, greatly supported RfP among villagers. The villagers enjoyed to read the 
newspapers and comment on the news together after their farming work in the late morning and evening. Female villagers also enjoyed to do craft together.

In addition, in urban poor areas where people still live in a rural, collective way, people go to share personal stories, give moral support to each other, and use books collectively in CL2. This evidence shows that despite the fact that marginalised people generally see RfP as a middle-class activity, as previously stressed by CL2's officer in 6.3.2.1, in fact, it is a universal activity for both the rich and the poor. However, the way RfP is practiced by the classes might differ. While the middle class can own books and enjoy reading individually, people in urban poor areas use books collectively.

CLs in extremely deprived areas often operate similarly to a childcare centre. CL2, CL4, CL6.1, CL7, and CL8 were very popular among children who came to join storytimes and other collective activities. Moreover, CL6.1 was not only a leisure reading space but a place to support educational opportunity and teach life skills for children in need.

\subsubsection{Public Libraries and Librarians}

Most participants referred to public libraries as formal and unfriendly, and not places that rural peasants commonly feel comfortable accessing. In most cases, villagers/people in urban poor area need to feel the library as informal so that they can wear their normal shabby clothes. Thus, CLs and SBHs are highlighted as venues which should not be luxurious or very formal or look like "a holy place" with a formal librarian that might intimidate disadvantaged people.

The DPL who received numerous awards for her outstanding efforts in linking the public library with CLs commented on the inadequate government support for CLs. She and other leaders in Cases 2 and 3 strongly insisted that if there are a variety of books provided to their communities, the villagers are eager to read. Participants agreed that CLs in any form could open up reading opportunities for disadvantaged people because the intense and a complex sense of inferiority of underprivileged people make them feel awkward in public libraries.

The popularity of the two CLs in urban poor areas in Case 1 and Boonta's comments about CL7 confirmed these comments. Although Boonta is an educated reader, and have 
more confidence than ordinary Isan students as mentioned in 9.3.1.1, as a girl from a village she does not feel comfortable using formal public libraries. "As a university student, I don't dare to use the big public library in the district. It looks very formal. I feel tense to use it. If I were a teacher, I would dare to use it" (CL7 Boonta). This reflects the high status of teachers that can transform an inferior-feeling rural girl into one who feels eligible accessing formal venues. Her comments also show that confidence is crucial for library access. The lack of confidence is the result of both low SES and the formality of libraries in Thailand that require respectable attire and fluency in the CT language.

The librarian's role as a "guardian of books" is highlighted by key participants. Even though the DPL is friendly, her words on truanting students who use the public library to relax in reflect a traditional belief that the librarian judges them, and does not welcome 'risky' people to relax in the space. The contrast between her friendliness and her judgemental attitude about 'risky' students highlights the importance of CLs and shows the nature of public libraries in Thailand that cannot fully serve as a welcoming safe places while they make judgments about those who enter. In the CL context, all participants mentioned that $\mathrm{CL}$ officers fully engaged with disadvantaged communities because CLs are small spaces in which rural people can interact with friendly library workers. The $\mathrm{CL}$ workers who are normally locals, create flat relationships with library users who are also locals from the villages.

\subsubsection{Education, Poverty, Isan identity, and Isan language}

Each case shows first, circumstances of extreme poverty and unequal educational opportunities that highlight the economic struggle affecting parenting, and second, the lack of time and resources within families to support RfP. All CLs provide opportunities for marginalised people who usually cannot access quality new books. The BSP in several CLs are an example of the creation of a newly introduced reading culture which is possible in rural areas, and inclusive for disadvantaged people.

Case 1 shows the contrasting picture of a middle-class Isan, on the one hand, who has self-confidence and takes his children to a public library and reads to his sons, and the people in urban poor areas on the other who exclude themselves from book access. This is because they are aware of their dirty clothes and their class and so feel uncomfortable 
in the public library space. They also do not borrow children's books that cost 100-200 baht because they worry that they cannot pay to replace books and think that the librarians must be very concerned with the condition of the library books. The scenarios show that people of lower status marginalise themselves from the right to access facilities, even when the library and books are aimed to support them.

Similarly, findings in all cases show that the impoverished rural Isan identity makes the people very humble and unconfident. Boonta's talk in the previous section reflects this extreme humbleness. The leader in CL7 talked about his Isan inferiority complex when he studied in a good university in Bangkok, and that he thought that the complex generally delays Isan people's personal development. The leader in CL8 supported the previous comments and Aree's words in 9.4.2 that rural Isan people and poor people are reluctant to be visible in public, even when doing something good.

However, several participants mentioned that some Isan individuals use disadvantage to drive themselves to seek achievements. The example is Naree (CL6) who was a slow learner and had no money to spend with friends so she went to libraries, and there the books that she read for pleasure changed her life. However, her family background was different from most Isan in that she had a progressive mother who was well-educated for her time. Moreover, the mother by chance introduced RfP to her and her sister from a young age. The most obvious element of the Isan identity is the Isan language, which Isan people who cannot speak CT properly identified as a factor making people from other regions look down on them. Thus, rural Isan people feel inferior and awkward in formal spaces (such as public libraries), and urban spaces. Because of this, participants mentioned that more Isan parents try to have their children speak CT from birth in order to increase their confidence.

In most cases, participants agreed that speaking Isan at home did not affect CT acquisition because the learning process in which teachers use both languages at school gradually helps Isan students to acquire CT. Nevertheless, it is not until the age of 11 or 12 that Isan students who speak Isan at home can speak CT fluently. However, the abbot commented that the policy of using $\mathrm{CT}$ as the only means of communication affects the literacy development in children who use local languages at home. 
Cases show that important factors which encourage people to learn CT easily is access to interesting CT reading material via the school library, appropriate reading behaviour, and being read to from a young age in CT. In Case 2, participants mentioned that delayed CT acquisition delayed reading development and RfP, and the school library is often the only place that children are exposed to $\mathrm{CT}$ reading materials.

\subsubsection{Community Participation}

Over the last ten years in CL1 and CL4, community participation had been strong. The CL officer confirmed the result was that people and CDCs in the community used the library often. Similarly, CL2 was also initially established through the volunteer spirit of people in the community who allowed the WA to set up shelves in their houses to serve community members. In several cases, the leaders mentioned that they involved children and users of all ages in the selection of books for the CLs. Thus, it is a way to include users in the $\mathrm{CL}$ acquisition process and make them have a sense of belonging.

All participants agreed that this sense of belonging could sustain CLs, and it is preferable that the library service is provided through the good care of community people who are employed by local administrative organisations. For example, the municipality in CL2 was also concerned that the $C L$ needed community participation. When the library was established, the authority first looked for people in the community to work with because they belong to the community and can communicate with people in the community more effectively. However, it proved difficult to recruit people in the community to help run the library because library work lacked job security.

In addition, in Case 3, the reason the leader in CL7 did not receive full support from local authorities, and was not trusted by local teachers, might come from the fact that he was away from this village for a long time so people no longer considered him as a local.

\subsection{Theme D: Active Roles of Key Individuals in RfP}

Theme $D$ is the most important theme emerging from the study because the CLs in the study tend to be individual-driven rather than system/policy driven. This major theme emerged from the data and is connected with all other themes. The study reveals that 
most CLs do not receive direct financial support from the national government, therefore, leaders in RfP need to work closely with other key individuals.

In each case, the active contribution of these key individuals affects RfP in CLs at different levels. The theme includes: 1 ) leaders in RfP; 2 ) reading models and training for BSPs; 3 ) family members; 4) teachers; 5) reading volunteers/reading leaders; and 6) other individuals.

\subsubsection{Leaders in RfP}

Leaders in RfP are individuals who generally have more extensive education than reading promotors in the villages. Also, they establish the intervention to solve the problems of the book access scenarios listed above. They try to eradicate the most influential factors hindering RfP in CLs, for example, literacy traditions, attitudes toward RfP, and noninclusive feelings in $\mathrm{CL}$ access and activities. Since they have a good education background and connections, they are able to provide educational support to RfP and acquire quality reading materials for the CLs. The fact that the CLs rely mostly on the leaders that make most CLs vulnerable because they can be closed any time when the leader loses interest in running the project or has to move on.

The leaders in RfP who initiated CLs in all cases are examples of RfP natives who truly understand reading enjoyment and FVR. Their outstanding characteristics are passion, commitment, and creativity. Leaders in Case 1 conveyed a strong passion to intervene in current reading practice in schools and the community. Findings show that with their strong commitment and creativity, these leaders play significant roles in initiating, fundraising for, and establishing the library.

An example in the government sector is Yada (CL5) who stressed that reading promotors need to work out of the 'government mentality' box. The DPL (CL 3), who worked beyond her job description, confirmed this notion. Yada initiated a BSP in a rural area and needed to withstand questions from her bosses and colleagues about BSP. She passionately expanded her small-scale BSP in a village to the provincial level, and gradually used her creativity to persuade other key individuals, for example, authors and school teachers, to engage in reading activities and help make reading and CLs relevant to the community. 


\subsubsection{Reading Models and Training for Bookstart Projects (BSPs)}

Reading models are a crucial element in reading promotion because findings show that they produce tangible outcomes in reading to young children that people can observe. Thus, they could inspire and change villagers' beliefs about literacy development. Even the DPL who was educated and trained by the WA was not aware of the full value of reading to young children until she saw that it worked with her grandchildren.

The leader in CL4 also confirmed the notion that a reading model, demonstration, and participation are key training techniques. Other participants added that the success of CL4 is significantly affected by the key training techniques, and other contextual factors such as the villagers' readiness, and a large collection of quality material provided by a charity.

In all, findings show that there are three steps in training for BSPs for family members, child caregivers, and teachers: 1) communicating by organising an event to explain the need to change attitudes and provide knowledge on ECD; 2) providing picture books for free; and 3) following up consistently with weekly home visits.

The first step needs to involve the community leaders and the reading models who were participants in the previous BSPs. Reading promoters need to raise awareness in training by organising an event through which to convey sufficient information about the impact of BSP on literacy development. Reading models, children below five who were able to recall words in picture books, are an important element identified in most cases. Their parents' reflections on literacy and behavioural development would act as a stimulus for new participants.

Next, the promoters need to give access to picture books right after the training. Book ownership highlighted the importance of BSP and ECLF in CLs 2 and 5. These projects make book ownership possible for each household with young children but they only initially reduce the gap in the book access opportunities mentioned by the WA expert. Leaders in most cases stressed that CLs need to provide a good range of books continuously. The final step is not discussed at length but all leaders in RfP mentioned the need for continuous stimulation for caregivers in rural areas bot in the policy and local levels. 


\subsubsection{Family Members}

The findings from all cases indicate that family members and their attitudes towards child-rearing are the most influential factors for encouraging RfP. Therefore, the absence of parents from the family greatly affects RfP since generally parents are book providers, role models, and storytellers. The severe impact on children of their parents' migration, creating dysfunctional families throughout Isan is discussed in 10.3.4 -10.3.6.

Findings show that few villagers owned books, partly through poverty, though in some cases this came from a lack of awareness of the benefits of book ownership. The consequence of low book ownership is a lack of reading behaviour, as mentioned by the library officer in CL2. After having a picture book collection in her house, being trained to work as a library officer, and having two small children, her behaviour had changed so that she now reads more.

The most important aspect of family members for RfP is that parents or close family members are the most influential role models. Adults in the family need to read books themselves so children can see the pleasure, the intrinsic reward that comes from reading. This approach also strongly encourages the freedom to search for more pleasure in reading, showing the intrinsic happiness to be gained from reading, and suggesting that RfP is a desirable activity. However, findings show that such practices are extremely rare in the communities studied.

The leaders mentioned that the reading promotors need to work on parents' perceptions of ECD and literacy development so that children have parental role models and understand that reading is an important part of life. In Case 1 a mobile library was used to create a dialogue on the connection between books and children with childcare teachers and rural people in communities in an effort to promote RfP.

A middle-class father's comments in Case 1 presents an example of a father as a reading model and the modern lifestyle that can hinder RfP in urban areas. Even for parents who know very well that reading to young children nurtures literacy development, their busy modern lives lessen their ability to support RfP for their children. The situation was more severe in impoverished families, as stressed by the WA expert in 6.3.1.3. Therefore, in low-income families where there are no books for RfP or there are uninterested parents, 
other people, for example, teachers and other adults in the community, need to be role models for the children.

\subsubsection{Teachers}

Half of participants agreed that Thai teachers hold traditional beliefs about the primacy of reading for academic purpose. For that reason, the WA expert in Case 1 said teachers are unlikely to be good at encouraging RfP and felt that even training would not change the teachers' approach. Moreover, the opposition of high school teachers to CL7's youth activity reflected the teachers' authoritarian mind-set. It seems that the teachers cannot accept the youths' personal development and other changes in society. However, numbers of participants mentioned that teachers have been gradually changing their attitude toward RfP. A document in CL2 reveals that once school administrators and teachers understand RfP, the impact is high as it could help reduce power distance.

The teacher-based learning style and vertical social structure that regards teachers as being of higher status than the villagers are shown by Boonta's comments in 10.4.3. The power distance issue was reflected in the lengthy comments by the reading volunteer in CL4. She asserted that a flat relationship is much appreciated by those lower in social status and will help support RfP in CLs. A class division between peasant/villagers (chao ban) who are less educated and professionals (chao nai) who are educated and superior can be seen from her perspective. Thus, interaction between the village reading volunteers and SOTs needs to be based on tactful interaction to bridge the class division.

A report in CL2 also proposes that teachers are the best reading models because teachers' reading interests inspire students to read, for example the classic Japanese book for reading promoters and ECD, Totto-chan, the Little Girl at the Window (by Tetsuko Kuroyanagi). Book talks are also mentioned by a teacher as a means to share reading among teachers.

As for training for teachers, the experiences of an ECT in CL7 confirmed the WA expert's notion that a key aspect of the learning tradition in Thai society hinders RfP; that Thai teachers stress academic knowledge. However, after she attended training, she was completely transformed from an ECD who stresses academic knowledge into an RfP native who has the capability to create handmade picture books and train others. 
There is an extreme contradiction in training opportunities in CL1. The abbot, who had the highest degree in the study, clearly understood the value of picture books and light reading as an important conduit to more demanding texts. However, his employee, a temple kindergarten caregiver did not know how to use picture books to their best advantage, showing that she has never been trained in ECD.

\subsubsection{Reading Volunteers/Reading Leaders}

Both the WA expert and Yada emphasised in 10.2.2 that reading volunteers are the most crucial factor enhancing RfP practice in the country. Their reflections identified the characteristics of such volunteers, who need to have strong minds, an ability to promote reading, a volunteer spirit, and leadership. However, volunteer work, which is a part of being an active citizen, is seen by many other Thais as an unusual activity, as mentioned in 10.3.2. Moreover, as pointed out in 10.3.9.1 the Thai culture does not encourage free opinion and leadership, so it is challenging to nurture reading volunteers/leaders.

Civic engagement and leadership programmes were encouraged by Happy Reading in CL6 and CL7. In CL8 the leader in RfP started the CL through youth engagement and leadership; however, it ceased operation after some years. There were a great variety of activities in CL6 and CL7 that empowered youth and local people to engage in reading and health issues. The NGO in CL6 initiated training to create 100 reading volunteer networks in schools and over 30 civil society organisations in the province.

In CL7, the NGO cooperated with schools in the district and was involved in a variety of activities using media channels to build social media communications on RfP. This resulted in a large impact for a small CL. Boonta reflected that she had been trained and received opportunities to practice important skills, for example training in storytelling principles and techniques of public speaking, leadership, and interpersonal skills. Other key characteristics of reading promoters mentioned were teamwork, tactfulness, talking and persuading skills, and soft skills needed to deal with children. This partly shows the process needed to create reading volunteers/leaders, but no participants suggested a method of identifying reading volunteers who can work long term for the CLs. 


\subsubsection{Other Individuals}

In Case 2, the study reveals that the major factors affecting the success of reading promotion in ONIE CLs, often the very first reading resources for disadvantaged people, are the local leaders in RfP, DPLs, SOTs, and ONIE reading volunteers. However, their ambiguous roles in the government system are likely to weaken ONIE CLs. In Case 3 VHVs are the most crucial reading promoters. Observation confirms that the volunteers VHVs who are usually local women aged around 50, actively support the BSP.

Overall, the findings suggest that there are many desirable characteristics for $\mathrm{CL}$ volunteers. First, the promoter needs to be local and a friendly person so they are familiar with the community environment and can attract library users. Second, they need to have a volunteer spirit and be pragmatic so they can go "the extra mile." This is because in deprived areas, circumstances can be extreme, as in the urban poor area mentioned in 6.5.4. Third, in cases which operate similarly to a CDC, the volunteer workers need to be able to care for young children, help with the children's homework, and read picture books to children. Last and most important characteristic as mentioned in Case 1 and Case 4, is having a knowledge of books and being readers themselves.

The findings show that opportunities to attend appropriate training and capability building make a difference for library workers. This can be seen from the nursery caregivers and library officer in CL1 who did not receive any training opportunities. In contrast, the CL officer in CL2 regularly received such opportunities and she wished other $\mathrm{CL}$ officer could have chances similar to hers.

\subsection{Chapter Summary}

The chapter synthesises results from the four case reports to present a holistic and higher level of interpretation of the factors affecting RfP reading promotion practice in CLs. The four key themes show how factors facilitate or hinder RfP reading promotion practice in the CLs. A conclusion including further discussion of these themes is presented in the next chapter. 


\section{CHAPTER 11 Conclusions}

The four themes presented in the previous chapter emerged from a comparison of the four cases studied and addressed the first and second research questions: what are the factors affecting RfP reading promotion practice in CLs in the Northeast region of Thailand, and how do these factors facilitate or hinder RfP reading promotion practice in the CLs? This chapter synthesises the major findings of the study, and further discusses each factor examined through the theoretical lenses described in chapters 2 and 3. Significant literature and updates on the ongoing situation relating to RfP practice and ECD in Thailand are also added to this chapter to reflect on the most current contributions to RfP practice, both in the Isan area, and in Thailand generally.

\subsection{Theme A: Provision of Book Access in Disadvantaged Areas}

To promote RfP for people in areas characterised by poverty, Krashen (2011a) identified the need to provide access to a great variety of reading materials with minimum censorship, and with an emphasis on the reader's freedom to choose books. Theme A represents the major function of CLs in the study: the provision of book access in urban poor and rural areas.

The theme encompasses the core of the Community Library Factors - reading materials, and most of the Thai Reading Context factors - the national reading policy, coordination between supporting organisations, the book market and book purchasing.

Unlike Theme B: Literacy Development Support that encompasses five Thai Cultural and social factors, the relationship between factors in Theme $A$ is simple and the contextual factors tend to impact on the provision of reading material evenly, as showed in Figure 11.1. However, book purchase is a factor that connects to attitudes towards RfP (Theme B), and also extreme poverty in some Isan families (Theme C). 
Figure 11.1 Relationship between factors in Theme A

\section{Thai Reading Context Factors}

\begin{tabular}{l|l|l}
$\begin{array}{l}\text { A.2 National } \\
\text { Reading Policy }\end{array}$ & $\begin{array}{c}\text { Community Library } \\
\text { Factors }\end{array}$ & Market and \\
& Book Purchase \\
& A.1 Reading Materials & \\
A.3 Coordination between Supporting Organisations
\end{tabular}

\section{A.1 Reading Materials}

All CLs in this study play a strong role in providing a variety and choice of reading materials for people in deprived areas, particularly children in rural and urban poor communities. Leaders in RfP found that both children and adults had an appetite for books and were eager to read but they did not have quality material with enough variety to suit their interests.

During the Smart Book House Project $(\mathrm{SBH})$, the results showed that newspapers were the most popular reading material in rural areas, supporting adult literacy, RfP, and civic engagement when the villagers discussed the news after work. Female villagers also enjoyed the opportunity to access magazines that provide general knowledge and crafting methods. All participants emphasised the severe impact of the serial budget cut, confirming the Ecological Framework's emphasis on responsiveness to local needs as a crucial aspect of $\mathrm{CL}$ management. When serial publications were withdrawn from the CLs, RfP reduced considerably in rural areas.

In relation to book donations, the Book Bank in urban CL6 tended to receive books of sufficient quality and the book exchange project operated well. However, similar projects in rural areas were unsuccessful due to the poor quality of books. ONIE's Book Voyage project received low quality donated materials from villagers. The poor quality 
of donated materials found in this study confirms other Thai studies (Ahmed, 2010; Leowarin, 2010; Kongrut, 2017).

As a result of the poor quality of donated material, in all the case study CLs focusing on young children, book donations were not accepted. Picture books were newly bought or published by the CLs. CLs also tried to find new approaches to increase book access in rural areas; for example, in CL7 there was a suggestion from a school teacher to encourage parents to buy new books for schools to offer to the monk as a form of merit making. This reinforces a PUBAT (2018) initiative to deter merit making through the donation of old, poor quality books that do not match rural people's reading interests.

On light reading materials, most participants understood FVR principles and its benefits. Thus, they supported the acquisition of comic books, magazines, easy reading books, and some more challenging books for people with different levels of proficiency and interest. At CL6, the RfP leaders (Naree and Nalinee) had read 1 baht Thai comics extensively at a young age supported by their mother, which had a positive effect on their attitude towards RfP. This worldview not only conforms with Krashen's assertions about 'junk' reading material being just as important as more worthy material for RfP, but also Ngamwittayaphong, Paweenayothin and Sae Chang's (2010) comments that variety in reading material is more important than quality. She explained that what is not perfect stimulates curiosity, and provides more space for multi-dimensional thinking and imagination. Her study identified that this also applied to the handmade books mentioned in three cases in this study. Participants found that children like handmade books both in oral literature projects (CL5), and picture book production projects (CL6, and CL7).

However, despite the RfP leaders' support for light reading materials, most of them mentioned and had experienced Thai parents' strict control over light reading materials for their children, which the leaders felt seriously hindered their RfP (Krashen, 2011a or b). Findings show the control originated from the parents' strong knowledge-oriented mind-set, their authoritarian approach, and their limited knowledge of RfP.

Most leaders mentioned that parents restricted comic reading, which contrasts with PUBAT's (2015) survey in 2015 finding that comics/graphic novel is the favourite reading 
genre for general Thais (34.4\%). Thus, edutainment comics which gained parental approval were found to be popular in most CLs. For example, "Stingy Family" Korean series, as explained by Tan (2011) and Karuchit and Thoongbai (2009), are originated from the ability of the Korean writers to transform academic topics into easy and entertaining comics.

\section{A.2 National Reading Policy}

Over the period of this study, "The Decade of Reading 2009-2018 Agenda" was in operation. The agenda positively affected the CLs under ONIE (CL3, and CL4) in terms of serial access from ONIE. CL6 and CL7 indirectly gained financial support through projects submitted to Happy Reading, which had been established under the agenda and greatly contributed to their success. Since Happy Reading (2015) aims to promote healthy living and strengthen civil society, activities in the CLs vigorously reflected these purposes and made them outstanding in terms of the variety and impact of the activities they organised at the district and provincial levels. The other key advantage the CLs gained was the training provided by Happy Reading.

However, most cases in this study reflected that the national reading agenda is an ineffective measure for sustaining reading behaviours in the long term and improving book access in the rural areas, urban poor areas, and small towns studied because it is based upon event-based reading promotion. The data reveal that in 2015 the agenda did not specify clear reading promotion practice and direction, which is similar to the situation Ngamwittayapong (2011) found during 2009-2011.

Participants contended that the government needs to provide more detail about good reading promotion practice, provide more budget for the ONIE CLS, and find a process to identify and create reading leaders/reading volunteers who can encourage new readers. However, there was conflicting data from Yada, a government official in CL5, who suggested that these individuals need to be local people who can start to promote RfP at a local level without waiting for government policy. Thus, in her experience, civic involvement and leadership on an informal basis are crucial for RfP in CLs. When looking in detail at the 2017 Reading Framework, this type of informality is also mentioned, 
which links with the research results relating to the importance of an appropriate atmosphere in CLs (Theme C).

There are several aspects identified by the 2017 Reading Framework (Department of Cultural Promotion, 2017) that may overcome the factors hindering RfP found in the study. First, the framework realises that the event-based reading promotion approach does not encourage sustained reading behaviours. However, in practice the President of PUBAT commented that MOC, the major ministry responsible for the framework has been granted only 10 million from the 30 million that the $\mathrm{MOC}$ requested from the government. That amount is enough just for an event, not continuous projects. In contrast, the South Korean government has started to promote reading with 1,600 million baht in the first year (PUBAT, 2018).

Second, the framework places great emphasis on cooperation between organisations, which is identified as a crucial factor in the study. It facilitated TK Park and Happy Reading operations that in early 2018 were proactively and strategically executing their core purpose to link 200 model CLs under four partnering agencies nationwide.

However, the Framework does not focus on reading enjoyment, the missing component in reading behaviour within the Thai population (TK Park, 2008; 2010). This is contrary to the participants' assertions that the government should raise public awareness about RfP because it is at the root of reading skills and reading behaviours. Moreover, the Framework uses "time spent on reading" to measure reading behaviour, not the variety of reading material that TK Park (2010) found to be the most important index to measure reading behaviour in Thai people.

\section{A.3 Coordination between Supporting Organisations}

The study reveals that coordination between supporting organisations is crucial both for book access in disadvantaged area, and expert knowledge to raise awareness of RfP among the general public. The leader in CL5 asserted that the reading agenda would be sustained if there could be more parties involved to form a network. Due to the uncoordinated variety of arrangements, the CLs studied were originally established through many different types of cooperation. The most active CLs were independent, run by NGOs or a government officer who worked similarly to an NGO (Yada, CL5). These 
CLs generally gained stable financial support from either Happy Reading or TAOs. This finding is similar to that of Håklev (2010a) who found the most successful type of reading gardens in Indonesia were the independent ones started by small NGOs or citizen groups. Similarly, the NGO CODE-Ethiopia (C-E) has established CLs in rural Ethiopia for more than 25 years (Asselin, Abebe \& Dorion, 2014).

The strong support given by Happy Reading to CL6 and CL7 resulted in their activities having a diverse and broad impact (presented in Case 3 and 4). However, in Case 2, the success of the ONIE CLs relied considerably on the ONIE retired executive (CL4), and the DPL who voluntarily worked beyond her job description. Case 2 revealed a close connection between ONIE SBHs and public libraries, and the ambiguous relationship between the DPL and SOTs which negatively affected the CLs' operations. This is a unique issue relating to the Thai government structure that, if resolved, might change $\mathrm{CL}$ operation for the whole country.

Findings show that Happy Reading worked as a catalyst to link education and public health systems by providing knowledge to local communities so that they can persuade TAOs to use the social security budget for establishing an ECLF in their communities. However, each TAO decides whether book ownership and storytimes for children are a priority. Overall, participants often commented that if TAO and ONIE could find a way to cooperate it would greatly enhance RfP efforts in Thailand.

\section{A.4 Book Market and Book Purchase}

The WA expert in Case 1 and Makut Ornrudee (Aimjai, 2001) had rather similar ideas about Thai reading promotion. They suggested that the government should change the focus from reading events and activities to the national book market structure by addressing it at policy level. Participants in every case also mentioned the shortage of children's books and bookstores in rural areas and small provinces.

The complex consignment system, the rise of digital piracy and wider use of social media have greatly affected the publishing industry in Thailand. Many bookstores have closed due to high rental cost, and declining numbers of customers. Even highly circulated magazines, for example, Koo Sang Koo Som and Kwan Reun, noted as popular among 
female villagers in the study, ceased publication at the end of 2017 after 38 and 49 years respectively.

Koo Sang Koo Som was frequently mentioned in the study due to its very affordable price and accessible content. It has been said it was "at one time a must-read magazine for most Thai households" (Jipleecheep \& Suvicha, 2017). Piracy problems were the major reason the publication shut down, confirming the views of an expert on the South East Asian publishing industry on the challenging situation facing magazine publishers in the region (International Publishers Association, 2014). It suggests that this Thai popular magazine, collected by the National Library of Australia for its social and educational value, cannot compete with online reading in the country (Trentacosti, 2015).

As for book purchasing, the study participants were of two worldviews. Most participants believe that people of any class will buy books to read for pleasure if they understand and see the benefit of RfP. However, leaders in RfP asserted that without intervention in the book industry, books are still expensive for most rural people. Thus, people's attitude towards RfP and purchasing power contribute to different RfP practices.

Most participants felt that the government should invest more in the provision of reading materials for rural areas. Even the former ONIE executive confirmed the inequality in access to reading materials, saying that rural people can normally access only dated donated books in ONIE CLs. The 2017 Reading Framework did not propose any intervention in the complex book market consignment system. Thus, the WA manager and Makut's request on this issue is still an ongoing agenda and was discussed amongst reading experts at a national book system seminar in 2018 (PUBAT, 2018).

\subsection{Theme B: Literacy Development Support}

Literacy development support is a major role of CLs because children's books are scarce in deprived areas. With reading activities (B.9) at the centre of the theme, Theme B predominantly encompasses contextual factors, the five Thai Cultural and Social Factors: B.1 Literacy Traditions; B.2 Normative Culture, Feminine Society, and Religion; B.3 Attitudes towards RfP; B.4 Picture books and Reading to Young Children; and B.6 Media; 
and two Thai Reading Context factors: B.7 Literacy Problems and MOE Literacy Policy; and B.8 School Libraries. These factor interacted with parents' migration and parenting (B.5), specific Isan Socioeconomic Influences as shows in Figure 11.2.

Figure 11.2 Relationship between factors in Theme B

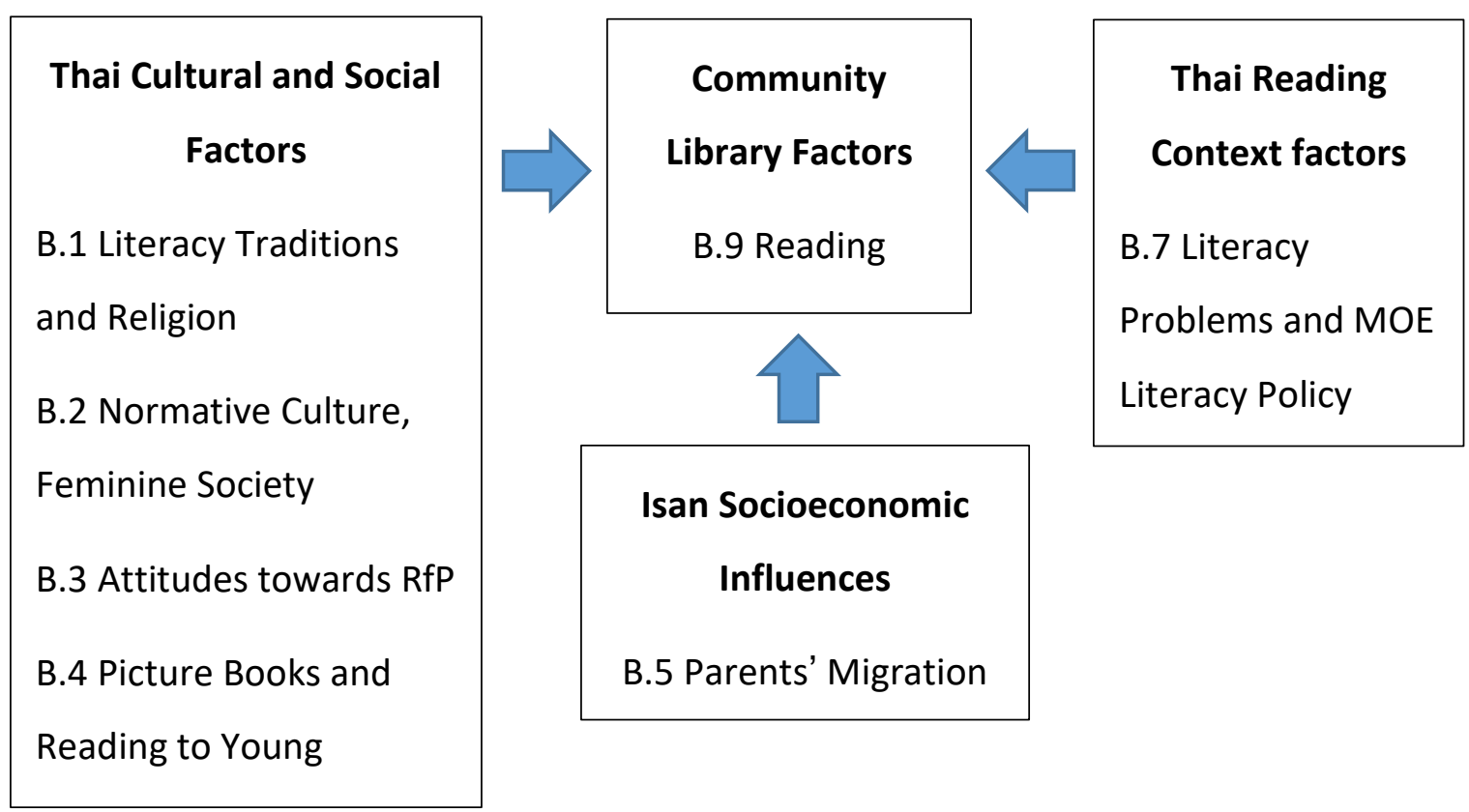

\section{B.1 Literacy Traditions}

The study finds that at least three literacy traditions radically obstruct RfP in Isan children from a young age. First, the teacher-based learning style encourages parents to avoid reading to young children and to leave the literacy development burden to CDCS and primary schools.

Second, the firm knowledge-oriented mind-set suggests that remembering and writing are the most important practice for the start of literacy development. This tradition, coupled with the availability of only Thai $A B C$ books in rural areas, makes most villagers and EDC teachers force young children to memorise and write the alphabet letters with no regard for maturity and readiness.

The third tradition is reading only for academic reading purposes, which is derived from the knowledge-oriented mind-set and the lack of knowledge of RfP due to the absence 
of books and reading activities in rural areas. This links to Theme $A$ because rural parents have never accessed books other than textbooks, and therefore have no idea what reading enjoyment is and what the benefits are.

The traditions were dominant in rural areas, thus, reading promoters said they needed to spend a lot of energy making adults aware of the benefit of RfP. The findings support those of an OBEC's (2011) paper, the 2017 reading framework, and arguments from two scholars, Eoseewong (2011) and Ngamwittayaphong, (2011), that formal education's excessive focus on 'knowledge' is the major cause hindering good reading behaviour by Thai children. The excessive and forceful literacy practice is confirmed by a prominent Thai psychologist, Dr. Prasert Palitpholkarnpim, who found an increasing number of children suffered from stress in classes (Thai Tribune, 2017).

These findings are key elements in a Thai ECD crisis identified by the Drafting Committee for Early Childhood Development (2017) and Preschool Education Association of Thailand (2018). Therefore, 2018 is a milestone in the development of ECD and literacy tradition, supported by Happy Reading as presented in 3.2.2.3. (Happy Reading, 2017a; 2017b).

The leaders asked for an awareness-raising campaign on reading and storytimes aimed at the general public, caregivers, and teachers. In addition, leaders in urban poor areas commented that a more explicit policy on the Early Childhood Literacy Fund (ECLF) at a national level would greatly equalise the opportunity of marginalised communities to access children's books. They felt that the ECLF should focus particularly on facilitating picture book access for the large number of Isan children who were left behind by their biological parents, or live with parents who have low SES. These children have an extremely limited chance of exposure to children's books. As reported by the National Statistical Office (2016), in 2016 the percentage of Isan children aged 0 - 5 with at least three children's books at home was $41.2 \%$, and the percentage of households with 10 or more children's books was $13.5 \%$.

\section{B.2 Normative Culture, Feminine Society, and Religions}

Findings in all cases show that the distinctive normative and feminine characteristics of Thai society tend to obstruct reading to young children, and voluntary work in CLs. These 
cultural aspects are further discussed in B.4 Picture Books and Reading to Young Children, and C.1 Community Participation and Civic Involvement

The results show that Thai adults both in rural and urban areas tend to value educational reading materials over books for RfP. The change of conventional thinking in reading culture are mentioned by Ngamwittayaphong, Paweenayothin and Sae Chang (2010), and a progressive TK Park (2018) book, Box: Library world, learning world, experience world, suggesting that to promote reading culture, Thai of all generations need to be pragmatic and adapt to the new fast-changing environment. The book suggests Thai adults should accept and encourage young people to think differently, to think outside the box, and not be obedient and deferential.

The strong knowledge-oriented mind-set and the WA expert's comment that classic picture books were largely absent from the Thai market contrasts with the situation in China, a pragmatic society (Hofstede Insights, 2018c). Tan (2011) argued that the Chinese embraces RfP because "Its children's segment is expanding and going beyond material with educational value. Picture books have a bigger audience than before, and the quality of titles in the marketplace has improved significantly".

In terms of religions, both Buddhism and Christianity contribute to RfP in CLs but in different ways. Two educated abbots directly involved themselves with RfP by providing space in two Buddhist temples for CLs. While these educated monks understand that education and social work are merit-making, a large number of Buddhists who have normative thinking seem to believe differently and do not support volunteer activities. Therefore, in most cases the library volunteers, both young and middle-aged, identified themselves as "strange" villagers who did not gain recognition from other villagers for their outstanding voluntary work.

Another aspect of Buddhism is the spiritual craft learned from books and training provided by SOT that brought great pleasure and additional income to the female villagers in CL4. It shows that this ONIE CL and RfP in rural areas can contribute to more aspects of life and then reading behaviour can serve as "central players in personal, social, cultural and economic improvement in their communities" (Asselin, Abebe \& Doiron, 2014, p. 5). 
Christianity was mentioned for providing educational support and progressive thinking in Isan areas. The Christian charity school in CL2's area supported the CL officer and her children. Similarly, CL5 and CL6 were located in two different provinces in Isan but revealed the influence of modern education for three generations, which positively affected RfP. In CL5, Nida, the reading model who regularly read to her granddaughter, attended the Christian private school in the village, and started to be a reader in an urban Christian school. In CL6 the leaders' grandfather, who loves to read, progressively sent their mother to a Missionary School. CL6 showed the influence of a liberal education in which both leaders were raised reading and doing social work. Naree was possibly the most advanced leader in RfP in the country because years before this study she had clearly identified that literacy traditions are the biggest challenge facing reading promotion.

\section{B.3 Attitudes towards RfP}

All study participants are RfP natives who have positive attitudes towards and an understanding of RfP. The participants identified the "forgetting" or "lost in the book" experiences that Krashen includes in his FVR principles. Leaders in CL7 and CL6 revealed that the happiness and enjoyment are derived from their reading supported their literacy development and overcame their low SES, confirming studies by the OECD (2010) and Sullivan and Brown (2013).

However, findings show that it is challenging to change the general negative attitude of Thai parents toward RfP. Two prominent leaders in Case 3 contended this is because 'reading' and 'pleasure' are two contradictory concepts for Thais. Most Thai people believe that reading is only for academic purposes, and pleasure connotes only entertainment. The 2017 Reading Framework tends to confirm the separation of the two concepts. It defines three purposes of reading: "for knowledge, for philosophical thinking, and for entertainment (to kill time)." Moreover, the low priority put on RfP in the Framework confirms Naree's assertion that this study could change perceptions about RfP in the country. The framework is going in a different direction to policy in other countries; RfP is one of the National Library of New Zealand's (2017) three strategic directions to 2030 , for example. 


\section{B.4 Picture Books and Reading to Young Children}

Picture books are the main reading material found in most CLs and they are used to engage parents and children with storytime sessions. Their beauty fascinated both children and adults, and they function primarily as a literacy development tool because they inspire affective feelings (delight, safety, warmth, etc.) that helps create lowanxiety situations and family bonding (McDonald, 2017).

Nida, who identified herself as a 'strange villager' was a keen reader and different to the majority of Thai people who believe that young children cannot understand language from picture books (PUBAT, 2015; MGR Online, 2015). Nida's engagement with her grandchildren, and other cases in nearby villages showed that reading to young children is easy. Also, she did what WA in Case 1 found to be effective: 1) caregivers need to read regularly and continuously to children, 2) caregivers need to read a good range of books. However, her case is in contrast to the findings in most cases in which parents care more about working to feed their family than allocating time for reading engagement and parenting. The WA expert pointed out that the gap in the opportunities for middle class and poor children to be read to was huge, which showed in MICS surveys mentioned in 3.3.1.3.

In terms of picture book access, the BSP project in CL5, which received copyright and a regular budget from the TAO to print and provide three picture books to families with new born babies, is reflected in the United Nations' (2002) agenda - A World Fit for Children. It contributed to noticeable literacy development and healthy living in the rural communities. Findings show that after the picture books were initially provided for free, the BSP motivated parents in the areas to acquire additional picture books, similar to the positive effect of other BSP in Thailand (MGR Online, 2015). The increase in children's book ownership is particularly important in Isan where the statistics from 2012-2016 showed the lowest percentage of children's book ownership in the country (National Statistical Office, 2012; 2016). Moreover, CL5 contributed to the successful BSP because it is a communal space where mothers can read to babies together, so they do not feel the practice is unusual. 
The WA expert's assertion on the rarity of picture books in rural areas is supported by other cases. In addition, as previously mentioned, literacy traditions and normative society hinder rural villagers reading to young children. Thus, in all cases, leaders in RfP need to put greater and more consistent efforts into promoting the practice.

\section{B.5 Parents' Migration and Parenting}

Parents' migration, and grandparents' limited financial resources are found to affect literacy development significantly. The findings show that nurseries and schools have to be responsible for literacy development, therefore $C L 2, C L 7, C L 8$ also function as informal childcare centres. The study confirms that low SES limited the opportunities of parents to engage in activities that support ECD (as mentioned B4).

The study shows that the quality of care that children receive from grandparents greatly affects RfP and school performance. Grandparents tend to have conservative literacy traditions and in some cases they do not allow their grandchildren to visit the CL. Moreover, they tend to support excessive materialism, for example smartphones, and tablets.

\section{B.6 Media}

Findings show that rural people, particularly grandparents, are open to children using electronic devices, particularly smartphones. Perhaps it is a symbol of modernity/urban sophistication that rural Thai people admire (Mills, 1997). At one point, mobile companies provided smartphones for free with data purchase in rural areas, and participants commented on the resulting excessive media exposure which confirmed the Child Watch project mentioned in 3.3.4. An admiration for young children who can use modern electronic devices was particularly prevalent among elderly and parents who reportedly felt that screen time made children concentrate and stay home. The unlimited screen time common in both rural and urban poor areas in the study is against the recommendations of both the Thai paediatric (Drafting Committee for ECD, 2017) and the Canadian Paediatric Society (2017) for no screen time for children aged 0-2, and less than an hour per day for children aged 2-5. 
Findings show that parents/caregivers tend to spend limited time communicating with their children, particularly when they are young. Smartphones, which babies and preschoolers can access freely, seem to keep children occupied when parents are busy but increase the chance that young children do not have appropriate literacy development. This is because young children need physical communication and interaction with people, a print-rich environment and free play (Adkins \& Brendler, 2015; Anderson \& Fearnley-Sander, 2015; Preschool Education Association of Thailand, 2018).

High book prices in the country, as discussed in A.4, make IT/digital devices appear a better investment in terms of reading, which is reflected in a study on mobile reading in Africa by UNESCO (2014), and the 2017 Reading Framework. It seems that the framework and preferences for reading material found in this study show a shift towards digital reading, which might be disruptive for literacy development in young children in rural Thailand. Reading applications containing quality picture books aimed at children, for example Ebookadabra (National Literacy Trust, 2017), could help young Thai children aged 3-7 avoid the negative effect of inappropriate media exposure mentioned in 3.3.4. Although social media was positively used to establish CL8 and to invite people to join the activities in CL6 and CL6.1, in most cases participants identified that media addiction in children and teenagers greatly affected public library and CL visits in Isan. The study does not focus on the problem but Young (2017) identified that the problem has been heavily studied in Asian cultures since the beginning of the century; the Korean government developed plans to deal with Internet addiction among its population, for example. With disruptive technology, participants thought that reading promoters need to think creatively about new ways to promote RfP via social media and smartphone.

\section{B.7 Literacy Problems and MOE Literacy Policy}

B.1 to B.6 discussed the many factors hindering literacy development in Thai children. The study finds that the way MOE handled the literacy problem during 2014-2015 was too demanding for both first graders and teachers. Leaders in RfP and teachers mentioned that the MOE's literacy policy needs more practical guidelines, interesting reading materials, and teacher involvement. 
Most leaders commented that the focus on reading skills and the traditional knowledgeoriented mind-set might, contrarily, be too forceful and hinder children's reading ability and attitudes toward reading. The high-anxiety situation is against FVR principles (Krashen, 2011a) and confirms TK Park's study (2008) that negative experiences caused by excessive reading demands at a young age are a cause of non-readers.

Another contextual consideration is that the content and stories in the standard Thai textbook are not relevant to Isan children. The disregard for cultural democracy in textbooks and education policy supports Fry and Bi's (2013) comment about overcentralisation in Thai education and the inclusion/equality aspect in the Ecological Framework.

To alleviate literacy problems, the traditional phonics approach was mentioned by a teacher. Also, exposure to picture books and lively storytimes mentioned by participants greatly supported literacy development which allies with Krashen's point that his FVR is not for early learners, and storytime is the first step for literacy development (see 2.3.)

Case 2 showed that the top-down, ill-prepared MOE early literacy policy had to be applied and evaluated in ONIE's SBHs less than a year after the command, resulting in the DPL's frustration. In addition, the early literacy policy may not gain much attention from SOTs because their primary role in rural areas is in adult education. Moreover, the DPL and active SOTs commented that the lack of picture books to use in SBHs from the district ONIE hindered the practice. This reflects back to A.1, A.4 and B.4, that the success of early literacy programmes depends on the initial provision of free picture books.

\section{B.8 School Libraries}

The findings showed that school libraries had a close connection with CLs. In all cases, CLs served school students, and the leaders in each CL engaged teachers and students in $\mathrm{CL}$ initiatives, particularly early childhood teachers and elementary school teachers. Therefore, teachers and teacher librarians were identified as important partners in most CLs, which is reflected in the Ecological Framework and Krashen's assertions on the importance of the school library for children in poverty. Naree (CL6) identified teacher librarians as important links between students and the library, parents, and freedom to enjoy RfP. The fact that there is no professional teacher librarian in primary schools 
greatly hinders library operations and support for literacy development. This longstanding problem of the lack of teacher librarians is identified by Pradit (2006).

Most participants stressed that school libraries are vital for both RfP and reading skill. However, they are generally under-resourced due to budget constraints, as seen in Wimolsittichai's (2017) recent study on rural small public primary schools, particularly those located in the far North and Isan regions. In addition, the Abbot in CL1 stressed that the most critical problem in Thai education is inequality in the schooling system; a reflection of the hierarchical Thai culture that favours and invests in only smart students, and excellent schools (Ippoodom, 2017).

\section{B.9 Reading Activities}

The core activities of the CLs studied were circulation services and storytimes. However, in CLs that had sufficient training opportunities, a large budget, and sufficient volunteers for example CL5, CL6, CL7, there was a large variety of activities organised by health promotors and VHVs. Book Bank (CL6) and BSP (CL5), with their focus on book access and storytimes, were the most noteworthy activities in this study.

Most of the CLs have informal circulation services and losing books from the library was common and not of serious concern. However, in CL2 the people in urban poor areas did not dare to borrow books because they were aware that picture books are expensive and that they cannot pay if the books were lost or damaged. Therefore, they marginalised themselves from book access and accidentally curtailed the opportunities of their young children to listen to stories and have the experience of owning picture books.

Control over book choice and the affective aspect of reading activities were mentioned as essential elements in RfP in all cases. However, participants who are RfP natives mentioned that most Thai parents and teachers are non-RfP natives so they normally control children and choose reading material for them, as discussed in A.1.

In terms of affective aspects of reading activities, the oral tradition which links to happiness in families and community, and pride in the Isan cultural heritage, was mentioned as a positive factor affecting RfP. Bedtime stories told by caregivers created 
family bonding and positive attitudes towards RfP. More importantly, folklore projects developed in a school library by the leader and a school teacher in CL5 not only successfully encouraged literacy development, but also encouraged curiosity, imagination, family bonding, and better social relations.

Social relationships in reading activity was identified by Ngamwittayaphong (2011) as a crucial element to create a reading culture, which she argued is more important than the creation of quality books. Tassanee's (CL4) view that activities with affective elements are vital for the creation of new readers, also supported the notion.

When participants said that music, storytelling, and poetic dialogues in traditional plays are RfP, it reveals that in an oral society, literacy can come in multiple forms. Most importantly, maintaining traditions would help the $\mathrm{CL}$ strengthen its vital role in community development in terms of "equity", focussed on cultural diversity in the Ecological Framework.

The WA expert's assertion on the need to create new readers was supported by most participants. Findings show that providing physical book access in any form of $C L$ will not be sustained if there is no active officer who has a basic understanding of how to undertake reading activities with children. However, in the case of BSPs, picture book provision is equally vital because villagers cannot find picture books in their areas and the eagerness to read after training will disappear if there are no books to read at home. Concerning Krashen's FVR point about rewards, most participants viewed reading as a reward in itself so did not see the need to give any extrinsic rewards to readers. However, the study reveals that rewards are used to draw new children to CLs and open opportunities for them to see good books which will lead to an interest in reading, plus intrinsic reward. Therefore, CL6.1 and CL7, CL8, organised competitions with fun peerinfluenced activities, and provided both intrinsic and extrinsic rewards, for example food, snacks, and stationery. This is similar to Africa where Asselin, Abebe and Dorion (2014) mentioned that library officers prepare food for important CL events.

Regarding accountability, the study shows that it is a problem in the ONIE system. Since the CLs studied were not involved in setting book reports after reading, only the teacher 
in CL5 commented on the balance between freedom, creativity, and accountability in school reading schemes.

\subsection{Theme C: Provision of Inclusive, Safe Atmosphere}

Theme C presents the social perspective, "inclusion/equity/social justice" identified by the Ecological Framework as one of its four core component for $\mathrm{CL}$ development. The framework focuses not only on community participation but also "diversity of culture and language-and all aspect of humankind" (Asselin \& Dorion, 2013, p. 132). Ngamwittayaphong's (2011) study also stressed a flat/equal social relationship as an essential element in reading activities.

Since most CLs in the study were children's libraries, the findings show that CLs have a significant role in providing relaxed, inclusive, safe spaces without judgment for disadvantaged children and adults. Such spaces were found to support community participation and civic involvement. Therefore, in Figure 11.3 this is in the centre of the theme with reading activities, because oral tradition activities create inclusiveness and the maintenance of cultural diversity, as discussed in B.9.

Theme C encompasses three overarching Thai Cultural and Social Factors: C. 2 Learning Atmosphere and Location; C.3 Sharing Reading; and C.4 Public Libraries and Librarians. Lastly, the specific Isan Socioeconomic Influences completed the theme, as shown in Figure 11.3.

Figure 11.3 Relationship between factors in Theme $C$

\begin{tabular}{|c|c|c|}
\hline Thai Cultural and Social Factors & $\begin{array}{l}\text { Community Library } \\
\text { Factors }\end{array}$ & $\begin{array}{c}\text { Isan Socioeconomic } \\
\text { Influences }\end{array}$ \\
\hline Location & C.1 Community & C.5 Education, Poverty \\
\hline C.3 Sharing Reading & $\begin{array}{l}\text { Participation and Civic } \\
\text { Involvement }+\end{array}$ & Isan identity, \\
\hline C.4 Public Libraries and Librarians & & and Isan language \\
\hline
\end{tabular}




\section{C.1 Community Participation and Civic Involvement}

With both high book prices and inequality in resource distribution in Thai society, CLs are a critical social intervention. Findings show that leaders in RfP in CL1, CL4, and CL6 all mentioned inequality in book access and library access for people from different SES. Therefore, they tried to intervene by encouraging community participation, and seeking external support from other organisations, as discussed in A.3. In March 2018, the President of the National Book Institute commented that since there had been no state mechanism to control book price, " $\mathrm{CL}$ is a crucial mechanism for book access [for the majority of Thais], not expensive learning centres in the luxury malls [in the city areas] which "cho bans'" (commoners') children have no chance to access" (PUBAT, 2018).

The findings are similar to the situation in Africa, in which Chisita and Chiparausha (2017, p.36) identified that CLs not only bring relevant reading material to underprivileged people, but their value "lies in their essential sense of social purpose...The primary stimulus is humanitarian, and its outcome is social intervention in support of positive prejudice".

Findings show that considerable community participation in the early period of the establishment of each CL created a sense of belonging which participants agreed was a factor affecting their sustainability. CLs engaged community members by: 1) supporting and training youth groups to undertake fundraising activities and participate in the interior design of the $\mathrm{CLS}(\mathrm{CL} 7$, and $\mathrm{CL} 8) ; 2$ ) inviting children and users of all age ranges to select books for the CLs (CL4, and CL5); 3) setting up bookshelves in volunteer houses to serve community members $(C L 2) ; 4)$ establishing picture book gifting projects Bookstart Projects (BSP) (CL4 and CL5).

In $\mathrm{CL1}$, the whole community used the $\mathrm{CL}$ because engagement was from most stakeholders in the community. In CL2, the volunteer spirit of Orada, who initially allowed the WA to set up shelves in her house to serve community members, resulted in the most-visited CL in all seven CLs from the same project. In CL7, and in CL8 the youth empowerment made it famous, since it was a rare initiative in the country.

In CL5's area, villagers showed that community participation and picture book access via a BSP can equalise literacy development opportunities among people in all physical 
circumstances. The deaf-mute parents' case showed that with support from people in the village, their daughter could speak and had appropriate literacy development. The activity showed that reading activity participation not only helps individuals achieve personal pleasure and skills but also means that family and community can connect (Duncan, 2013). This is also similar to the African collective culture, which has a saying "It takes a whole village to raise a child" (Doiron \& Lees, 2009; Rhaiti, 2016).

CL5 was the most advanced case in this study encompassing all components in the Ecological Framework. Yada, strategically combined her Master's degree research, village health volunteers (VHVs), academic connections, and the TAO to support her BSP, which was not her in job responsibility. The case showed the power of civic involvement by VHVs, mostly middle-aged female villagers who gained community trust more readily than Yada and her public health officers. This confirms Kowitt, Emmerling, Fisher, and Tanasugarn's (2015) finding that community trust is the PHV's strongest point.

In terms of civic involvement, the distinct extreme feminine and normative Thai character was confirmed by participants as factors hindering volunteer work and reading to young children, as discussed in B.2. A youth in CL7 and several library volunteers who engaged in those activities identified that other villagers judged them as "strange". Findings reflect distinctive low Masculinity, and Long-Term Orientation in the Thai culture (score 34 and 32). Another hindering factor for civic involvement may be one of the Thai traditions mentioned in 3.3.1.1 and B.1, that only teachers are authorised to train students. Therefore, teachers may feel their power being challenged by both the leader in CL7 and students who were more confident after joining activities in CL7.

One factor missing from the CLs in the study was a Library Management Committee with members nominated from community people. Observation showed that all CLs were informal organisations with no planning and management, which is different from the successful CODE-Ethiopia's CLs (Asselin, Abebe \& Dorion, 2014).

\section{C.2 Learning Atmosphere and Location}

All CLs were found to provide a non-judgemental, inclusive, safe atmosphere for children in deprived areas. The CLs functioned as a "safe space for people" not a "safe space for things", similar to the way the first free public libraries in the North American colonies 
operated, in contrast to the libraries in Europe guarding valuable manuscripts for nobles (Wexelbaum, 2017).

In $\mathrm{CL2}, \mathrm{CL} 5$, and $\mathrm{CL8}$, participants said the villagers were comfortable using the CLs in their regular shabby clothes, which are typically shorts and flip flops, even though it is considered impolite to enter official venues in Thailand in such clothes. In 2016 a lecturer was not allowed to enter her university library because of her knee-length shorts, showing that rigid library rules are still strictly practiced in Thailand (Bangkok Post, 2016). The news stirred comments on libraries as a holy "closed space" which prevents people enjoying being in learning spaces in Thailand in general (Ippoodom, 2017). The incident reinforces the finding that disadvantaged people are strongly concerned about their attire when entering libraries outside their communities.

The service providers who provided a relaxing atmosphere encouraged library visits, and helped create community dialogue. When villagers feel they are equal, safe and not judged in a $\mathrm{CL}$, they share conversations. CLs are small spaces in which rural people can interact freely. The place with no restriction on book access and socialisation is, in fact, the concept of a library as a Third place in civic society (Oldenburg, 1999). This helps create the FVR low anxiety situation and supportive physical and social atmosphere (Asselin \& Doiron, 2013).

Regarding location, most CLs studied were not separate buildings but residences, shops or community properties that were at the centre of their communities, easy to access, and provided a physically and psychologically relaxing atmosphere. Users were able to sit and read at any time. The traditional seating arrangements, and freedom to relax in the $\mathrm{CL}$ space were crucial in most cases. Physically, villagers liked informal seating arrangements in which they could gather to read a daily newspaper and discuss news. Most villagers were happy to sit and read on mats or the traditional low day beds provided. In Case 2, ONIE SBHs were flexible enough to relocate to a new location when the old ones were unsuitable. The fundamental problem was the location, to which SOPs/volunteers could not deliver the daily newspaper on a regular basis. In CL5, VHVs who volunteered their house to be a book hub also distributed and recommended books to villagers. 


\section{C.3 Sharing Reading}

Findings show that group gatherings and the opportunity to share reading brings enjoyment, nurtures an individual love of reading, and creates community bonding, which is similar to the collective and oral nature of Malaysian society (Pandian, 2011) and African societies (Chisita \& Chiparausha, 2017). In all cases, CLs provide both initial opportunities to share reading, and later opportunities to read voluntarily and independently when individuals have developed their reading interests, as predicted by the Ecological Framework.

Sharing reading was mostly seen in newspaper reading in ONIE SBHs (Case 2) mentioned in A.1 and picture book reading for mothers in the BSP at CL5. In Case 2, serials that SBHs had access to for only two years (2014-2015), especially newspapers and magazines with craft sections, greatly supported RfP in the villagers. The people like to read newspapers and comment on the news together after their farming work in the late morning and evening. Female villagers also liked to do crafts together at CL4.

Also, in the urban poor area where people still live in a rural, collective way, people went to CL2 to share personal stories, give moral support to each other, and use books collectively. This evidence shows that despite the fact that marginalised people generally see RfP as a middle-class/high-class activity, as previously stressed by the CL2 officer in 6.3.2.1, in fact it is a universal activity for both the rich and the poor. However, the way RfP was practiced by the classes might be different, based on the ability to buy books.

Observational and interview data in all cases revealed that sharing reading with peers at the CLs generated support and a fun feeling of competition for children. The positive peer influence and users' freedom to select books of their choice was mentioned as enhancing RfP in most contexts.

\section{C.4 Public Libraries and Librarians}

The study shows that people who perceived themselves as villagers prefer to use informal CLs rather than official district public libraries, which confirmed TK Park's study (2010). This may be because public libraries tend to be a heteronormative space, not an inclusive space (Wexelbaum, 2017). Participants agreed that CLs in any form could 
facilitate reading opportunities for disadvantaged people because the intense and complex sense of inferiority among underprivileged people (see C.5) makes them feel too awkward to enter a public library. Most participants referred to public libraries as formal, unfriendly, and thus intimidating to disadvantaged people. Even Boonta (CL7), a university student said she did not dare access a public library.

Findings show that confidence is crucial for library access. The confidence is relevant to both SES, and the formality of libraries in Thailand that require formal attire and CT language, as mentioned in $\mathrm{CL2}, \mathrm{CL} 4, \mathrm{CL} 6$, and CL8. Thus, CLs and SBHs were mentioned as venues which are not luxurious or formal with stern librarians who leaders in RfP at $\mathrm{CL1}, \mathrm{CL} 5$, and CL8 stereotyped as the guardian of the library.

The librarian's role as a 'guardian for books' as reflected by key participants showed that librarians need to revisit their approach to engaging with the community. Even though the DPL (CL3) was friendly, her comments about truanting students who used the public library to relax reflected a traditional judgemental attitude, which may make certain people feel unwelcome in the space. The contrast in approach between $\mathrm{CL}$ and public libraries highlighted the importance of CLs and showed that because of the nature of the public library in Thailand, it cannot adequately serve as a welcoming, safe place.

All participants mentioned that the library officers need to engage with disadvantaged communities. The findings showed that a DPL in the study put great efforts into linking the public library with CLs. However, she commented on the inadequate government support. These findings are reflected in both the Ecological Framework, and Cheunwattana's (1998) comments that the public library system in Thailand needs greater community involvement and empowerment.

\section{C.5 Education, Poverty, Isan identity, and Isan language}

Isan socioeconomic influences, particularly poverty and education, are relevant to book access, literacy development, and inclusiveness because they are the primary factors that make Isan people stay in disadvantaged areas and receive unequal resource distribution. As a result, the people migrate to work in Bangkok and major cities and leave their children with grandparents. Every case in the study showed circumstances of poverty and unequal educational opportunities, highlighting that economic struggle 
affected parenting, and, therefore, the time and resources available to Isan families to support RfP.

The BSPs in several CLs are the example of a creation of the newly introduced reading culture which was possible in rural areas, and inclusive for disadvantaged people. These key finding highlight Themes $A, B$, and $C$.

The Isan language is the most outstanding feature of Isan identity. However, Isan people who cannot speak Central Thai (CT) properly identified it as a cause that makes them feel inferior and awkward in formal spaces.

The oral literature projects and handmade picture books found in three CLs which contributed to book access, literacy development, and social bonding may help with identity maintenance and cultural inclusiveness, a crucial factor affecting the success of CLs. Several cases revealed how the Isan oral tradition could engage the elderly and children via shared storytelling and writing in oral literature projects in the CLs. Findings reveal that children and adults in the communities liked the handmade craft items produced from these projects, and the handmade picture books created in three CLs. These projects not only contributed to family bonding and literacy development but also cultural inclusiveness and participation.

Asselin and Doiron (2013, p.133) found that in CLs around the world handmade materials "are the most meaningful and popular". This is because they support local knowledge, ethnic identity, and language which will establish a strong base and support for individual $\mathrm{CL}$ and community development. 


\subsection{Theme D: Active Roles of Key Individuals in RfP}

Since the CLs in the study tended to be driven by individuals, here the Key Individuals in $\mathrm{RfP}$ is a major theme connecting with all other themes.

Figure 11.4 Relationship of factors and themes in the study

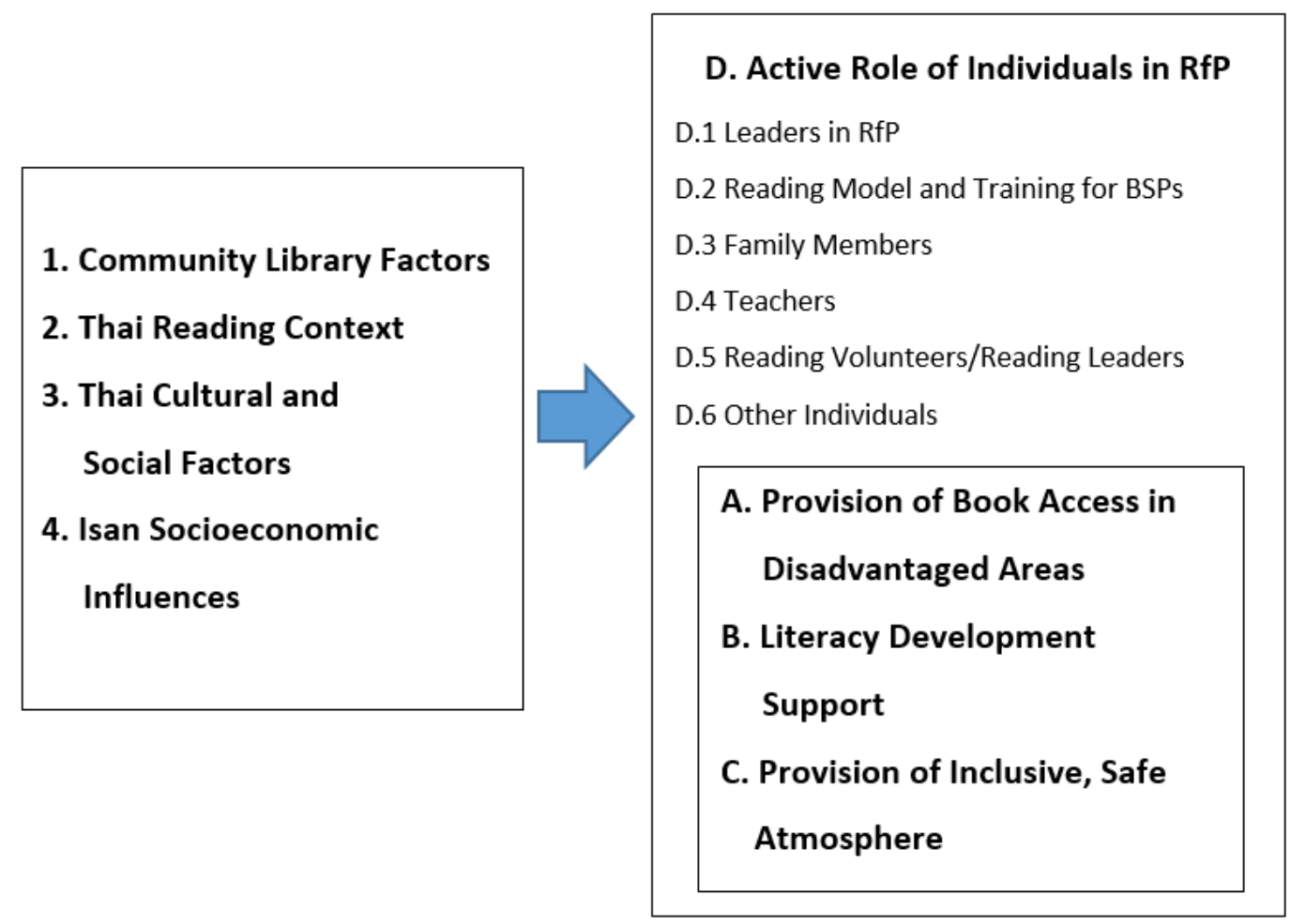

In each case, the active contribution of key individuals affects RfP in CLs at different levels. The theme includes: 1) Leaders in RfP; 2) Reading Models and Training for BSPs; 3) Family Members; 4) Teachers; 5) Reading Volunteers/Reading Leaders; and 6) Other Individuals.

\section{D.1 Leaders in RfP}

Leaders in RfP are individuals who generally have a higher education than the reading promotors in the villages and will intervene to solve the problems in book access mentioned in Themes A, B and C. They work on expert knowledge to eradicate the most influential factors inhibiting RfP in CLs, for example, unhelpful literacy traditions, negative attitudes toward RfP, and feelings of exclusion in $\mathrm{CL}$ access and activities. 
The leaders in RfP who initiated CLs in all cases are examples of RfP natives who truly understand reading enjoyment and FVR. Their outstanding characteristics are passion, commitment, and creativity. Leaders in Case 1 conveyed a strong passion to change the disparity of educational elements affecting RfP in the country, suggesting that there is a need to intervene in current reading practice in schools and the community. With their strong commitment and creativity, findings show that these leaders play significant roles in initiating, fundraising, and acquiring new materials for most CLs.

\section{D.2 Reading Models and Training for Bookstart Projects (BSPs)}

Reading models were identified as a crucial element in reading promotion in most cases because the children below five who are able to recall every single words in picture books are a tangible observable outcome of literacy stimulation by listening to stories in picture books. Thus they could inspire and change villagers' beliefs about reading to young children, literacy development, and ECD. Even the DPL who was educated and trained by the WA did not believe it until she saw that it worked with her grandchildren. The reading models, demonstration, and participation, are key training techniques. Other contextual factors, for example the villagers' readiness, and a collection of quality materials, also contribute to training success. Findings show that there are three steps in training for BSP for family members, child caregivers, and teachers: 1) communicating by organising an event to influence attitudes and provide knowledge on ECD; 2) providing picture books for free; and 3) following up consistently by weekly home visits. The first step needs to involve community leaders and the reading models who were participants in the previous BSPs.

After that, CLs continuously need to provide a good range of books and stimulation /training in rural areas. In addition, to increasing awareness about reading, several successful reading promotion models/strategies should be communicated to the public.

\section{D.3 Family Members}

Findings show that family members are book providers, role models, and storytellers, and the absence of parents from the family greatly affected RfP. However, only a few villagers were able to own books. Consequently, they lacked regular reading behaviour, 
which confirms Dickenson's study (2014) that SES and parents' education greatly affects RfP.

A contrasting scenario in book access was that of a middle class father who had seen his father read, was a reader himself, was a role model in reading, and can access libraries without worries. In low-income families, however, other people need to be role models for the children, for example, teachers and adults in the community. Oral literature, as mentioned in Theme $\mathrm{C}$, and other literacy programmes involving senior volunteers reading with school students (Doiron \& Lees, 2009) can make a difference to RfP, and encourage intergenerational communication and social bonding.

Having family members as role models is critical; most cases showed that participants had adult role models in their families and their children read because children can see the pleasure, the intrinsic reward, that adults can get from reading. However, findings showed that such families are extremely rare in rural and urban poor areas.

\section{D.4 Teachers}

Local teachers are key partnership targets identified by Asselin and Doiron (2013). Although this study involved few teachers, findings confirm Happy Reading (2017b) revealing that in rural Thailand teachers and education administrators are important stakeholders for creating a reading culture. Document analysis in CL2 revealed that when school administrators and teachers understood RfP, the impact was high.

Most leaders said that Thai teachers hold knowledge oriented mind-sets, are authoritarian, and do not have good reading behaviour, so they are unlikely to be good at encouraging RfP. Moreover, the teacher-based learning style and vertical social structure create power distance between them and villagers. However, SOPs in the study showed that they understand this aspect and try to create flat relationships with villagers. The villagers were very appreciative when class divisions between the peasants/villagers (chao ban) and the professionals (chao nai) were lessened.

Even though the WA expert found that training may not help teachers, the early childhood teacher case in CL7 showed that quality training can change a Thai teacher who used to focus on academic knowledge to be a RfP native. She was greatly inspired 
by Happy Reading training sessions and she was more confident, developing her capability to be a trainer in handmade picture books.

\section{D.5 Reading Volunteers/Reading Leaders}

Leaders in many cases emphasised that having reading volunteer/reading leaders is the most crucial factor enhancing RfP practice in the country. Their reflections identified desirable characteristics of such volunteers who initially need to have strong minds, voluntary spirit, and leadership. Civic engagement and leadership programmes were evidently encouraged by Happy Reading in CL6, CL7 and partially CL8.

Findings show that an ability to promote reading can be acquired through training. In $\mathrm{CL7}$, the youth reflected that she had been trained to have the key characteristics of reading promoters, for example teamwork, tactfulness, talking and persuading skills, and soft skills needed to deal with children.

In addition, the findings suggest that $\mathrm{CL}$ volunteers at least need to be local, and friendly, have a knowledge of books, and be readers themselves. In CLs which operate similarly to CDCs, the library workers need to be able to care for young children and help with children's homework.

Reflections from participants showed that CL staff in the study tended to "reframe their identity and role from guardians and authorities of resources to that of learners, leaders, promoters and researchers" as suggested by Asselin, Abebe and Doiron (2014, p. 8) although there was only one $\mathrm{CL}$ that supported research, CL5.

In all, the findings show that the identification of reading leaders/reading volunteers in communities is vital for CLs. Then they need to have opportunities to attend appropriate training. This can be seen from the nursery caregivers and library officer in CL1 who did not receive any training opportunities, whereas in contrast, the $\mathrm{CL}$ officer in $\mathrm{CL} 2$ regularly received opportunities, and she wished other $\mathrm{CL}$ officer had similar chances.

Although most leaders asserted that it is preferable that the $\mathrm{CL}$ service is managed by community people, $\mathrm{CL} 2$ showed that job security was a hindering factor in finding $\mathrm{CL}$ officers. This is rather similar to the Ethiopian CLs where Asselin et al. (2012) found that retention and training are challenges. 


\section{D.6 Other Individuals}

In ONIE CLs, the active roles of the local leaders in RfP, DPLS, SOTs, and ONIE reading volunteers contribute to CL success. However, their ambiguous roles in the government system is a factor weakening ONIE CLs. In CL5, VHVs are the most crucial reading promoters. In Case 4, community leaders and local governments committee were mentioned as influencing parents' attitudes to RfP.

\subsection{Research Contributions}

\subsubsection{Contributions to Theory}

The study facilitates understanding and clarification of RfP theory and community librarianship within the LIS community. In so doing, it redefines Duncan's explanation that RfP "usually denotes an individual, silent activity" (2013, p. 5). The findings reveal that RfP in the rural Thailand context aligns with Clark and Rumbold's (2006, p.5) definition stated in 2.2.1. but the study finds that RfP in the Thai context may not suit Duncan's (2013) explanation because, in rural areas where people still live a highly collective life, RfP is unlikely to be an individual, silent activity. Most reading activities for adults in rural Thai villages involve pleasure gained from socialisation. The fact that appropriate CLs need to be community meeting places also supports reading in a collectivist and inclusive manner, which is similar to RfP in Malaysia (Pandian, 2011) and Indonesia (Sugiharto, 2011). In addition, villagers read for pleasure and read to suit their purposes, for example to learn how to do handicrafts, which improve their fruit productivity and gain additional income. Thus, RfP in CLs in Thailand contributes to social, recreational, and economic purposes in deprived areas.

This study confirms that most aspects of Krashen's FVR are practiced among Thai reading promotors. However, extrinsic rewards such as food, snacks, stationary, and other rewards given in competitions, are mentioned as tools to initially attract new readers. After readers attend reading activities and are exposed to variety of books and find their reading interests, they would find that the intrinsic reward, reading enjoyment, is more encouraging than extrinsic rewards. Therefore, the FVR framework is not fully applicable in the Thai context and possibly other similar environments. In rural Ethiopia, Asselin, 
Abebe and Doiron (2014, p. 8) found that in order to seek partnership to increase interaction between CLs and communities, community librarians invite students and local people to join book clubs, and special events involving food and beverages.

This study confirms that Asselin and Doiron's (2013) Ecological Framework for Community Library Development and their five effective practices in reading promotion and literacy development (2013) (Table 2.2) are mostly applied in the Thai CLs. However, most of the CLs under investigation were not strong in terms of Library Management Committee roles. Consequently, many CLs had not efficiently operated in terms of staffing, training for CLs officers, engaging meaningful partnerships in the community, and seeking coordination for continuing budget.

The study expands our knowledge and understanding of models of community libraries which can be established by different organization/individuals. However, due to the particularistic, descriptive, and heuristic nature of multiple case study research, the findings of this study can be transferred to cases with a similar context but cannot be used for generalisation.

\subsubsection{Contributions to LIS Research and Methods}

The study applied sociocultural perspectives, not commonly found in reading promotion research (Asselin \& Doiron, 2013), and found complex, interrelated factors affecting RfP in CLs in the Isan context. This case study research yields deep understanding about community libraries in Isan in Thailand and their contextual factors that quantitative research cannot provide.

With a mixture of participants from different age, careers, educational background, and social status, the study revealed both the similar and different worldviews they perceived. The study shows that even though education is a status marker in the Thai society, educated participants who perceive themselves as "chao ban" or commoners still feel they are of lower status and dare not enter public libraries. Therefore, the factors found in the study are not simplistic nor linear, which is different from Hoq's (2015) comments on CLs studies. 


\subsubsection{Contributions for Practice}

For LIS practice, the attitudes and flat relationships found in the study question the traditional library service in the country. Therefore, library professionals will have a deeper understanding of approaches that can make libraries more relevant to the majority of Thai people who are low on the socio-economic ladder but have an eagerness to access books and read for pleasure.

The study makes suggestions at the policy level for prominent factors: the unclear reading policy; event-based reading promotion strategy; and insufficient investment in reading materials. More explicit policy on the ECLF, and the provision of more quality materials for CLs and school libraries would equalise the opportunity of marginalised communities to access children's books.

Most importantly, the 2017 National Reading Framework which covers 2017-2020, needs to focus on intervening in the complex consignment system in the book market, identifying and developing reading leaders, and raising awareness about RfP, new learning approaches, and media literacy.

Since the Reading Framework was only implemented in early 2018, it takes time to establish whether it fulfils the participants' hopes in terms of enabling cooperation between organisations. The point of concern is that there are 31 organisations involved with the promotion of a reading culture for Thailand as presented in 1.2.1. Currently in 2018, TK Park and Happy Reading are actively coordinating parties involved in reading promotion. However, they have no authority over any of the parties.

\subsection{Future Research}

\section{Partnerships between CLs and Schools in Rural Areas}

Since the study finds in two cases that schools tend to be a crucial element in literacy development for children in poverty, effective partnerships practice between CLs and schools in rural areas is an interesting topic that deserves further investigation. The topic should reflect a community participation element in the Ecological Framework (Table 2.2), suggesting local teachers as target partners for CLs. 


\section{Literacy development in Isan Student aged 6-10}

A question arising from this study is how and why children who speak the Isan language at home normally struggle for the first five critical years for literacy development at school, aged 6 to 10 . The double negative effect is that if they cannot enjoy CT material in those years, or before ages $11-12$, the children have very few chances to become readers because their puberty years is the period when teenagers often divert their reading habits to other activities. Future research on factors affecting literacy development in Isan students aged 6-10 could contribute to deeper understanding of students who learn different languages at home and school.

\section{Digital Reading in Young Children, Primary School Children and Pre-teenagers}

The study identified the smartphone as a key device that diverts children from reading when they become teenagers. Children spending time reading from smartphones fits Krashen's Surfing for Pleasure concept (2011). In addition, the 2017 National Reading Framework tends to support digital reading materials, which seems to be cost-efficient. Therefore, studies on digital reading behaviours of children in different age groups would contribute to the body of knowledge in LIS, and practice in public library and CL services.

\section{CL as "the Third Place"}

Utilising FVR and the Ecological Framework as lenses, the study shows the complexity of social factors, particularly low-anxiety and an inclusive, safe atmosphere which is in fact the core of modern public library service (Wexelbaum, 2017). The study accentuates the importance of CLs as comfortable spaces in which people can gain social acceptance, social interaction, relaxation, and companionship. Therefore, future research can investigate Thai CLs as "the Third Place," shared informal public gathering places that Ray Oldenburg (1990) stressed as vital elements in community and public life.

\section{Utilization and Interaction of Extrinsic and Intrinsic Rewards in Reading Promotion}

The study finds that extrinsic rewards is a particular aspect of Krashen's FVR that has not been fully practiced. Therefore, an investigation of the utilization and interaction of two types of awards received in reading activities/RfP could add to the body of knowledge on effective reading promotion activities that lead to RfP in Thailand. 


\section{References}

Abu, R. (2014). Community development and rural public libraries in Malaysia and Australia (Doctoral dissertation, Victoria University, Melbourne, Australia). Retrieved from http://vuir.vu.edu.au/24833/1/Roziya\%20Abu.pdf

Adkins, D. (2009). Reading sources and reading spaces in Honduras. IFLA Journal, 35(3), 215-225.

-------., \& Brendler, B.M. (2015). Libraries and reading motivation: A review of the Programme for International Student Assessment reading results. IFLA Journal, 41(2) 129-139.

Ahmed, S.M.Z. (2010). Measuring performance and impact of rural community-led library initiatives in Thailand. Information Development, 26(1), 17-35.

Aimjai, K. (2001). It's time for Thailand to have the National Book Institute: An interview with Makut Ornrudee. Retrieved from http://www.bflybook.com /Article /InterviewMakut1/InterviewMakut1.htm

Alemna, A. A. (1995). Community libraries: An alternative to public libraries in Africa. Library Review, 44(7), 40-44.

Anderson, P., \& Fearnley-Sander, M. (2015). The conditions of reading acquisition in contexts of low literacy. Retrieved from https://research.acer.edu.au/cgi/ viewcontent.cgi?article $=1023 \&$ context=monitoring_learning

Asselin, M., Abebe, A. \& Doiron, R. (2014). Applying an ecological model for library development to build literacy in rural Ethiopian communities. Paper presented at IFLA WLIC 2014. 16-22 August 2014, Lyon, France.

-------, \& Doiron, R. (2013). Linking literacy and libraries in global communities. London, England: Ashgate.

Ball, J. (2010). Enhancing learning of children from diverse language backgrounds: Mother tongue-based bilingual or multilingual education in the early years. Paris: UNESCO.

Bangkok Post. (2016). Library dress code prudish. Retrieved from https://www.Press reader.com/thailand/bangkok-post/20160824/281835758117140

Baumann, J. F. \& Duffy, A. M. (1997). Engaged reading for pleasure and learning: A report from the National Reading Research Center. Athens, GA.: National Reading Research Center.

Bird, K., Hattel, K., Sasaki, E, \& Attapich, L. (2011). Poverty, income inequality, and microfinance in Thailand. Manila, The Philipines: Asian Development Bank.

Blake, V.L.P. (2003). Research methods in LIS dissertations. Encyclopaedia of Library and Information Science (pp. 2513-2523). New York, NY: Miriam Drake. 
Boonaree, C., \& Tuamsuk, K. (2013). Community learning resources management practices in Thai Buddhist monasteries. Paper presented at the 2 nd International Conference on Integrated Information (IC-ININFO 2012), Budapest, Hungary.

Braun, V., \& Clarke, V. (2012). Chapter 4 Thematic analysis. In H. Cooper (Editor-inChief) APA Handbook of research methods in Psychology: Vol. 2. Research designs (pp. 57-71). Washington, DC: APA.

Bowers, K. Howard, V. Brown, A. (2018). Storytime in a box: The cooperative approach to preschool storytime planning children \& libraries. The Journal of the Association for Library Service to Children 16(1), 23-29.

Brinkhoff, T. (2018). Thailand: Regions and major cities. Retrieved from https://www. citypopulation.de/Thailand-Cities.html.

Butdisuwan, S. (2000). Reaching out through a mobile library. Retrieved from http://www.ifla.org/IV/ifla66/papers/099-175e.htm.

Burke, M.E. (2007). Making choices: Research paradigms and information management: Practical applications of philosophy in IM research. Library Review, 56(6), $476-484$.

Campbell, C. (2014). If there's going to be a Thai Civil War, Isaan will be its front line. Retrieved from http://time.com/2948172/thailand-isaan-province-identity/\# 2948172/thailand-isaan-province-identity/

Canadian Paediatric Society. Digital Health Task Force. (2017). Screen time and young children: Promoting health and development in a digital world. Paediatrics \& Child Health, 22(8), 461-468.

Carnoy, M., \& Rothstein, R. (2013). What do international tests really show about U.S. student performance? Retrieved from http://www.epi.org/publication/usstudent-performance -testing/

Changket, S. (2011). Development of community library: A model for the promotion of lifelong learning. (Doctoral dissertation, Silpakorn University, Bangkok, Thailand). Retrieved from http://www.thapra.lib.su.ac.th/objects/thesis/fulltext /snamcn/Somjintana_Changket__Doctor/abstract.pdf

Changsorn, P. (2015). Education reforms critical for the Thai economy, says World Bank. Retrieved from http://www.nationmultimedia.com/business/Education-reforms -critical -for-the-Thai-economy-sa-30261548.htmlChapman, J. W., \& Tunmer, W. E. (2015, July). The literacy performance of ex-Reading Recovery students between two and four years following participation on the program: Is this intervention effective for students with early reading difficulties? Paper presented at the 39th Annual Conference of the International Academy for Research in Learning Disabilities (IARLD), Vancouver, Canada. 
Cheunwattana, A. (1998). Information for rural development: A multiple-case study of library and information services to the rural communities in Thailand. (Doctoral dissertation, Indiana University)

(1999). Delivering and promoting library services in rural Thailand. Retrieved from http://www.ifla.org/IV/ifla65/papers/023-114e.htm.

- (2003). Mobile and outreach library services in Thailand. Information Development, 19(1), 23-27.

Chiengkul, W., Lertwicha, P., Chamreon, J., Yunphan, K., Panyachan, P., Kongsomboon, R. (2000). 100 recommended books for Thai children. Bangkok: Quality Learning Foundation.

Chisita, C. T., \& Chiparausha, B. (2017). Part 1: Africa 2 Public Libraries in A. Ismail (Ed.), Global Library and Information Science (pp.11-42). Berlin, Germany: De Gruyter Saur.

Cho, K.S., \& Krashen, S. (2016). What does it take to develop a long-term pleasure reading habit? Turkish Online Journal of English Language Teaching, 1 (1), 1-9.

Choi, H., \& Krashen, S. (2005). Hooked on comic book reading: How comic books made an impossible situation less difficult. Knowledge Quest, 33(4), 32-34.

Chongsatitwatana, K. (2011). Thailand publishing industry. Retrieved from http:// studylib.net/doc/5356027/thailand-publishing-industry-presented-by-kimchongsat...

Clark, C., \& De Zoysa, S. (2011). Mapping the interrelationships of reading enjoyment, attitudes, behaviour and attainment: An exploratory investigation. London, England: National Literacy Trust.

-------- \& Douglas, J. (2011). Young people's reading and writing an in-depth study focusing on enjoyment, behaviour, attitudes and attainment. London, England: National Literacy Trust.

------- \& Rumbold, K. (2006). Reading for pleasure: A research overview. London, England: National Literacy Trust.

Connaway, L. S., \& Powell, R. (2010). Basic research methods for librarians. Santa Barbara, CA: Libraries Unlimited.

Cooper, K., \& Stewart, K. (2013). Does money affect children's outcome? A systematic review. York, England: Joseph Rowntree Foundation.

Creswell, J. W. (2013). Qualitative inquiry and research design: Choosing among five approaches. 3rd ed. Washington DC: Sage.

Cuesta, J., \& Madrigal, L. (2014). Equity in education expenditure in Thailand.

Development Policy Review, 32(2), 239-258. 
Dechwittayapon, C. (2015). Externalising reading culture practice. Retrieved from http://www.komchadluek.net/news/edu-health/212263 (In Thai).

Dent, V. F. (2006). Modelling the rural community library: Characteristics of the Kitengesa Library in rural Uganda. New Library World, 107(1220/1221), 16-30.

Deolalikar, A. B. (2002). Poverty, growth, and inequality in Thailand. Manila, The Philippines: Asian Development Bank.

Department of Cultural Promotion. (2017). The draft of the National Learning and Reading Frameworks. Retrieved from http://www.culture.go.th /thai/index.php? option =com_content \&view=article\&id=3343:2015-08-28-04-22-42\&catid=34: news \& Itemid=351 (In Thai)

Deveney, B. (2005). An investigation into aspects of Thai culture and its impact on Thai students at an international school in Thailand. Journal of Research in International Education 4(2): 153-171.

Dickenson D. (2014). Children and reading: Literature review. Retrieved from http://www.australiacouncil.gov.au/workspace/uploads/files/research/children -and-reading-literatur-5432557e418db.pdf

Doiron, R., \& Lees, J. (2009). It takes a village to raise a reader: Reflections on an Intergenerational Literacy Program. School Community Journal, 19(1), 137-154.

Dorner, D. G., \& Gorman, G.E. (2011). Contextual factors affecting learning in Laos and the implications for information literacy education. Information Research 16(2), 1-23.

Drafting Committee for Early Childhood Development (2017). ECD in Thailand the end of "No Man Land." Retrieved from https://www.matichon.co.th/news/559051 (In Thai).

Draper, J. (2010). Inferring ethnolinguistic vitality in a community of Northeast Thailand, Journal of Multilingual and Multicultural Development, 31(2), 135-147.

------. (2015). Draft constitution neglects minority rights of millions. Retrieved from https://isaanrecord.com/2015/04/23/draft-constitution-neglects-minorityrights-of-millions/.

-------., \& Nilaiyaka, A. (2015). Culture and language promotion in Thailand: implications for the Thai Lao minority of introducing multilingual signage. Asian Ethnicity, 16(2), 203-234.

Duncan, S. (2013). Understanding reading for pleasure for emerging adult readers. London: National Research and Development Centre for adult literacy and numeracy (NRDC). 
Ekachai, S. (2017). How our education sustains dictatorship. Retrieved from http://www. bangkokpost.com/opinion/opinion/1255906/how-our-education-sustainsdictatorship.

Eoseewong, N. (2011, August 24-25). Reading habits of Thai people in cultural dimension. Paper presented at Thailand Conference on Reading 2011, Bangkok, Thailand.

Fletcher, J. (2017). What can we do to support reading for young adolescent students? Education. 45(2), 258-271.

Flouri, E., \& Buchanan, A. (2004). Early father's and mother's involvement and child's later educational outcomes. British Journal of Educational Psychology, 74 (2), 141-153.

Frederickson, T. (2016). Thai education fails the test while Singapore and Vietnam excel. Why? Retrieved from http://www.bangkokpost.com/learning/advanced /11632 40/thai-education-fails-the-test-while-singapore-and-vietnam-excel-why-

Fry, G., \& Bi, H. (2013). The evolution of educational reform in Thailand: The Thai educational paradox. Journal of Educational Administration, 51(3), 290-319.

Global Library and Information Science. (2017). A. Ismail (Ed.) Boston: De Gruyter Saur.

Gorman, G.E., \& Clayton, P. (2005). Qualitative research for the information professional: A practical handbook. London: Facet.

Guba, E. G. (1981). Criteria for assessing the trustworthiness of naturalistic inquiries. Educational Communication \& Technology Journal, 29(2), 75-91.

Guba, E. G., \& Lincoln, Y. S. (1994). Competing paradigms in qualitative research. In N. K. Denzin \& Y. S. Lincoln (Eds.), Handbook of qualitative research (pp. 105-117). Thousand Oaks, CA: Sage.

Haglund, L., \& Olsson, P. (2008). The impact on university libraries of changes in information behavior among academic researchers: A multiple case study. Journal of Academic Librarianship, 34(1), 52-59.

Håklev, S. (2010a). Factors that contributed to the community library movement in Indonesia. Libri, 60(1), 15-26.

--.-. (2010b). Community libraries in Indonesia: A survey of government-supported and independent reading gardens. Library Philosophy and Practice, 3(1).

Retrieved from http://digitalcommons.unl.

Hallinger, P. (2010). Making education reform happen: is there an 'Asian' way? School Leadership \& Management, 30(5), 401-418.

-----, \& Lee, M. (2011). A decade of education reform in Thailand: Broken promise or impossible dream? Cambridge Journal of Education, 41(2), 139-158. 
Hayes, D. P., \& Ahrens, M. (1988). Vocabulary simplification for children: A special case of 'motherese'. Journal of Child Language, 15,395-410. Happy Reading. (2015). About the programme. Retrieved from http://www.happy reading.in.th/about/ (In Thai).

Happy Reading. (n.d.). Babies and their First Book. Bangkok, Thailand: Happy Reading. (In Thai).

--.-. (2017a). Building Thailand 4.0 by reading to young children. Bangkok, Thailand: Happy Reading. (In Thai).

--.-. (2017b). Growth mindste: 11 routes in community reading power. Bangkok, Thailand: Happy Reading. (In Thai).

. (2017c). Smart Kids Smart Reading Home. Bangkok: Happy Reading.

Hart, G. (2010). New vision, new goals, new markets? Reflections on a South African case study of community library services. South African Journal of Libraries and Information Science, 76(2): 81-90.

--.-. (2011). The "tricky business" of dual use school community libraries: A case study in rural South Africa. Libri, 61(3): 211-225.

-.-. (2012). Moving beyond "outreach": Reflections on two case studies of community library services in South Africa. Retrieved from http://sajlis.journals.ac.za/pub/article/viewFile/26/31

Hempenstall, J. (Ed.). (2016). Read about it: Scientific evidence for effective teaching of reading. Retrieved from https://www.cis.org.au/app/uploads/2016/07/rr11.pdf

Hjørland, B. (2005). Empiricism, rationalism and positivism in library and information science. Journal of Documentation, 61(1), 130-155.

Hofstede Insights. (2018a). Country comparison. Retrieved from https://www.hofstedeinsights.com/country-comparison

----. (2018b). National culture. Retrieved from https://www.hofstedeinsights.com/models/national-culture/

- (2018c). China. Retrieved from https://www.hofstede-insights.com/countrycomparison/china/

Hoq, K. M. G. (2015). Rural library and information services, their success, failure and sustainability: A literature review. Information Development, 31(3), 294-310.

IFLA. (2007). Developing cultures of literacy: An International research project preliminary report. Retrieved from http://archive.ifla.org/VII/s33/project/ DevelopingCulturesLiteracyRep.pdf 
International Encyclopaedia of Information and Library Science. (2003). $2^{\text {nd }}$ edition. Southeast Asia (600-612). London: Routledge.

International Publishers Association. (2014). Southeast Asia's book market: An interview with Stan Gunn, Edge Ventures. Retrieved from https://www. international publishers. org/images/news/2014/sout-east-asia-book-market.pdf Ippoodom, T. (2017). Thai education: The Discrimination of low performance students. Retrieved from https://thematter.co/pulse/inequality-in-thai-education/25936.

Ismail, S. (2014). Factors affecting the implementation of information literacy education in Malaysian primary school. (Doctoral dissertation, Victoria University of Wellington, Wellington, New Zealand).

Jampaklay, A., Vapattanawong, P., Tangchonlatip, K., Richter, K., Ponpai, N., \& Hayeeteh, C. (2012). Children living apart from parents due to internal migration (CLAIM). Bangkok: Institute for Population and Social Research, Mahidol University.

Jipleecheep, P., \& Suvicha, N. (2017). Sun setting on renowned print players. Retrieved from www.bangkokpost.com/business/news/1385142

Jirapornkul, S., \& Yolles, M. 2010). Assessing values and value change in Thai organizations. Journal of Organisational Transformation and Social Change, $7(3), 321-347$.

Jongudomkarn, D., \& Camfield, L. (2006). Exploring the quality of life of people in North Eastern and Southern Thailand. Social Indicators Research 78, 489-529.

Jonjoubsong, L. (2008). An Integrated knowledge management model for community enterprises: A Case study of a rural community enterprise in Thailand. (Doctoral dissertation, Victoria University of Wellington, Wellington, New Zealand) Retrieved from http://researcharchive.vuw.ac.nz/handle/10063/796

Karuchit, W, \& Thongbai, N. (2009). Thai knowledge cartoon: History, current situation, and approaches for development. Bangkok: TK Park.

Keyes, C. F. (1989). Thailand: Buddhist kingdom as modern nation-state. Bangkok, Thailand: Duangkamol.

. (2003). The politics of language in Thailand and Laos. In M. Brown \& S. Ganguly (Eds.), Fighting words: Language policy and ethnic relations in Asia (pp. 177210). Cambridge, MA: MIT Press.

--.- (2014). Finding their voice: Northeastern villagers and the Thai state. Chiang Mai, Thailand: Silkworm Books.

Kongrut, A. (2012). Bucking a trend: A Local publishing house refuses to jump on to the e-book bandwagon. Retrieved from http://www.bangkokpost.com/print/287196/ 
Kongrut, A. (2017). Let's develop a culture of reading. Retrieved from www.bangkok post.com/opinion/opinion/1184985/lets-develop-a-culture-of-reading.

Kosonen, K. (2005a). Education in local languages: Policy and practice in South-East Asia. Retrieved from http://www2.unescobkk.org/elib/publications/first _language/first_language.pdf.

. (2005b). Vernaculars in literacy and basic education in Cambodia, Laos and Thailand. Current Issues in Language Planning, 6 (2), 122-142.

Kowitt, S.D., Emmerling, D., Fisher, E.D., \& Tanasugarn, C. (2015). Community health workers as agents of health promotion: Analyzing Thailand's Village Health Volunteer Program. Journal of Community Health, 40(4), 780-8.

Krashen, S. D. (1993). The power of reading: Insights from the research. Englewood, CO: Libraries Unlimited.

- (2004). The Power of reading: Insights from the research. 2nd ed. Englewood, CO: Libraries Unlimited.

---... (2011a). Free Voluntary Reading. Santa Barbara CA: Libraries Unlimited.

- (2011b). Protecting students against the effects of poverty: Libraries. The NERA Journal, 46(2), 17-21.

----. (2013). The hard work hypothesis: Is doing your homework enough to overcome the effects of poverty. Multicultural Education. 2O(3/4), 21-23.

----- . (2014a). Why invest in libraries? Retrieved from http://skrashen.blogspot. co.nz/2014/02/why-invest-in-libraries.html

---.. (2014b). The Common Core. Knowledge Quest, 42(3), 36-45,

--.-. (2016). The Purpose of education, Free Voluntary Reading, and dealing with the impact of poverty. School Libraries Worldwide. 22(1), 1-7.

-----. (2018). Do libraries and teacher librarians have the solution to the long-term English language learner problem? California School Library Association Journal, 41(2), 16-19.

-----., \& Ujiie, J. (2005). Junk food is bad for you, but junk reading is good for you. International Journal of Foreign Language Teaching, 1(3), 5-12.

-----.., Lee, S., \& McQuillan, J. (2012). Is the library important? Multivariate studies at the national and international level. Journal of Language and Literacy Education, $8(1), 26-38$. 
Kressmann, J. (2017). More than 90\% of Internet users in Thailand use smartphones to go online: Urban regions have higher usage rates. Retrieved from https://www. emarketer.com/Article/More-than-90-of-Internet-Users-Thailand-UseSmartphones-Go-Online/1015217

Lao, R. (2013). Voice in the wilderness. Retrieved from http://www.bangkokpost.com /print/375335/

Lathapipat, D., \& Sondergaard, L. M. (2015). Thailand - Wanted: A Quality education for all. Washington, D.C.: World Bank Group.

Leowarin, S. (2010). Community learning centres in Thailand. Retrieved from https:// www.dvv-international.de/adult-education-and-development/ editions/aed742010/experiences-from-asia/community-learning-centres-in-thailand/

Liamputtong, P. (Ed.). (2014). Contemporary socio-cultural and political perspectives in Thailand. Heidelberg, Germany: Springer Netherlands.

Lincoln, Y. S., \& Guba, E. G. (1985). Naturalistic inquiry. Beverly Hills, CA: Sage.

Manuel, J., \& Carter, D. (2015). Current and historical perspectives on Australian teachers' reading practices and preferences. Australian Journal of Language and Literacy, 38(2), 115-128.

Mardis, M. A., \& Everhart, N. (2014). Stakeholders as researchers: A multiple case study of using cooperative inquiry to develop and document the formative leadership experiences of new school library professionals. Library \& Information Science Research, 36(1), 3-15.

Martin, E. R. (2006). Team effectiveness in academic medical libraries: a multiple case study. Journal of the Medical Library Association, 94(3), 271-278.

Mason, B., \& Krashen, S. (2017). Self-selected reading and TOEIC performance: Evidence from case histories. Shitennoji University Bulletin, 63, 469-475.

Maxwell, J. A. (2013). Qualitative research design: An Interactive approach. (3rd ed). London: Sage.

McCargo, D., \& Hongladarom, K. (2004). Contesting Isanness: Discourses of politics and identity in Northeast Thailand. Asian Ethnicity, 5(2), 219-234.

McDonald, D. (2017). How prison mums can still read their kid a bedtime story. Retrieved from https://www.stuff.co.nz/entertainment/books/93969097/howprison-mums-can-still-read-their-kid-a-bedroom-story

Merriam, S. B. (2009). Qualitative research: A Guide to design and implementation. San Francisco, CA: John Wiley \& Sons. 
MGR Online. (2015). Paediatrician speaks about the belief of Thai parents on reading to young children. Retrieved from http://www.manager.co.th/QOL/ViewNews. aspx?News ID =9580 $000142063 \& \mathrm{Html}=1 \&$ TabID=1\& (In Thai).

Mills, M. B. (1997). Contesting the margins of modernity: Women, migration, and consumption in Thailand. American Ethnologist, 24(1), 37-61.

----.., Huberman, A. M., \& Saldana, J. (2014). 3rd ed. Qualitative data analysis: A methods sourcebook. Thousand Oaks, CA: Sage.

Ministry of Culture, Ministry of Education, \& Ministry of Internal Affairs. (2017) The Reading Culture Promotion for Thai Learning Society Framework 2017-2020 (B.E. 2560-2564). Bangkok: Ministry of Culture.

Mostert, B. J. (1998). Community libraries: The concept and its application with particular reference to a South African community library system. International Information \& Library Review, 30, 71-85.

Myers, R. L. (2005). The Isan Saga: The inhabitants of rural Northeast Thailand and their struggle for identity, equality and acceptance (1964-2004). (Master of Arts in Asian Studies, San Diego State University, USA).

Na Mahachai, S., \& Thoopkrajae, V. (2007). Reading habit 'way behind.' Retrieved from http://nation multimedia.com/2007/10/18/headlines/headlines_30052875.php

National Economic and Social Development Board. (2011). The Eleventh National Economic and Social Development Plan B.E. 2555 - 2559 (A.D. 2012 - 2016). Retrieved from http://eng.nesdb.go.th /Portals/0/news/plan/eng/THE\%20 ELEVENTH\%20NATIONAL\%20ECONOMIC\%20AND\%20SOCIAL\%20DEVELOPMEN T\%20PLAN\%282012-2016\%29.pdf

National Library of New Zealand. (2017). National Library of New Zealand: Positioning for the future: A Nation of readers. Retrieved from https://www.govt.nz/browse /engaging-with-government/consultations-have-your-say/national-library-ofnew-zealand-positioning-for-the-future/a-nation-of-readers/

National Literacy Trust. (2017). A new approach to digital reading: A Blog by Tom Grayson, co-founder of Ebookadabra. Retrieved from https://medium.com /national -literacy-trust/a-new-approach-to-digital-reading-8e49e7d4cbfd

National Statistical Office. (2008). Summary of the Survey on Thai reading behaviour B.E. 2551. Bangkok: The Office. (In Thai).

---.- (2010). The Reading of population survey 2009. Bangkok: National Statistical office. (In Thai).

----. (2012). Executive summary: The 2011 Survey on reading of the population. Bangkok: The Office. 
National Statistical Office. (2013). Monitoring the situation of children and women: Thailand Multiple Indicator Cluster Survey (MICS) 2012. Bangkok: National Statistical Office.

--.-. (2014). Summary of the survey on Thai reading behaviour B.E. 2556. Bangkok: The Office. (In Thai).

--.-. (2016). Monitoring the situation of children and women: Multiple Indicator Cluster Survey (MICS) 2015-2016. Bangkok: National Statistical Office.

Ndumu, A., \& Mon, L. (2018). An investigation of the experiences of Nicaraguan Costeño librarians. IFLA Journal, 44(2). Advance online publication. doi.org/10.1177/0340035218764496

Ngamwittayaphong, A., Paweenayothin, G., \& Sae Chang, K. (2010). Factors affecting the promotion of a reading culture in Thailand: Case study of Reading Culture Promotion Programme. Bangkok, Thailand: Thai Health Promotion Foundation . (2011, August 24-25). Factors affecting the promotion of a reading culture in Thailand. Paper presented at Thailand Conference on Reading 2011, Bangkok, Thailand.

Nimkannon, O. (2006). Libraries of the future today. Retrieved from http://www. bangkokpost.com /education/site2006/nf2my1606.htm.

OBEC. (2011, August 24-25). Thailand reading promotion policies: Overview, problems, and development plans. Paper presented the 2011 Thailand Conference on Reading, Bangkok, Thailand. Retrieved from https://www.tkpark.or.th/stocks /extra/000bdb.pdf

OECD. (2010). PISA 2009 Results: Learning to learn - student engagement, strategies and practices (Volume III). Retrieved from http://dx.doi.org/10.1787/978926 4083943-en.

Ohio Literacy Resource Center. (2015). What is family literacy? Retrieved from http:// literacy.kent.edu/familyliteracy/whatisit.html

Oldenburg, R. (1999). The great good place. New York, NY: Da Capo Press.

ONIE. (2014). ONIE Strategies and emphasis for fiscal year 2015. Bangkok: ONIE. (In Thai).

Online Teachers and Educational Professionals Association of Thailand. Early Childhood Curriculum B.E. 2560 (2017). Retrieved from http://www.kruthai.info/5589/

Pandian, A. (2011, August 24-25). How Malaysian reads: Home and school initiatives. Paper presented at Thailand Conference on Reading 2011, Bangkok, Thailand.

Pholkaw, N. et al. (2009). Report on "Let's Read to Children Project." Bangkok, Thailand :Thailand Research Fund. (In Thai) 
Phosri, N. (2014). A synthesis of research on/reading in Thailand published between B.E. 2544 to B.E. 2553 (2001-2010). (Master's thesis, Khon Kean University, Khon Kean, Thailand). (In Thai).

Pickard, A. J. (2013). Research methods in Information. 2nd ed. London: Facet.

PISA Thailand. (2015). PISA 2015 Result. Retrieved from http://pisathailand.ipst.ac.th/ news/pisa2015result

Pradit, A. (2006, September $20-24)$. Reading promotion in primary schools of Thailand. Paper presented to the International Board of Books for Young People Congress. Macau. Retrieved from http://www.ibby.org/index.php?id=671

Preschool Education Association of Thailand (2018). Holistic ECD: ECD Crisis and solutions. Bangkok: The Association.

Pribesh, S. Gavigan, K., \& Dickinson, G. (2011). The Access gap: Poverty and characteristics of school library media centers. The Library Quarterly 81(2), 143-160.

Promotion of Healthy Lifestyle Program. (2014). Media trends in Thailand, 2014-2021. Retrieved from http://mediamonitor.in.th/archives/10970

PUBAT. (2015). Reading and book buying behaviours of the Thai population. (2015). Bangkok: PUBAT.

---.-. (2018). Salvation or death?: Future of the country and the government with no national book system. Retrieved from https:// pubat.or.th/archives/1040

Pyati, A.K., Kamal, A.M. (2012). Rethinking community and public space from the margins: A study of community libraries in Bangalore's slums. Aear, 44(3), 336-343.

Rajchagool, C. (n.d.). Tambon Administration Organization: Are the people in the Dramatis personae or in the audience? Retrieved from http://citeseerx.ist.psu. edu/viewdoc/download?doi=10.1.1.562.9252\&rep=rep1\&type=pdf

Rakyutidharm, A. (2014). Chapter 32 Patronage, Dominance, or Collaboration? Thailand's NGOs and the Thai Health Promotion Foundation. In P. Liamputtong (Ed.) Contemporary socio-cultural and political perspectives in Thailand ( $\mathrm{pp}$. 517-531). Heidelberg, Germany: Springer Netherlands.

Ramasut, N., \& Rohitsatien, B. (2016). Office of the Minister Ministry of Education News: Reading policy. Retrieved from http://www.moe.go.th/websm/2016 /mar/125. html (In Thai).

READ Global. (2015). READ Nepal recognized for best practices in literacy - Library of Congress. Retrieved from http://www.readglobal.org/blog/181-library-of-congress

Rhaiti, A. (2016). 'It Takes a whole village to raise a child' - An African saying. Retrieved from http://www.rightforeducation.org/all-topics/culture/it-takes-a-wholevillage/ 
Sacchanand, C., Prommaphan, B., \& Sacchanand, S. (2008). Performance evaluation of public libraries in Thailand according to the public library standards. TLA Research Journal, 1(1), 1-16. (In Thai).

Save the Children Fund. (2014). Read on. Get on: How reading can help children escape poverty. London: Save the Children Fund.

Schwartz, R. M. (2015). Ideology and early literacy evidence: A Response to Chapman \& Tunmer (2015). Retrieved from https://readingrecovery.org/wp-content/ uploads/2016/12/chapman_tunmer_2015_response.pdf

Setthamalinee, S. (2010). A Case study of New Generations: The role of education in raising Chinese Muslim in Northern Thailand. Retrieved from http://gulen conferences.com/wp-content/uploads/2014/04/Suchart-Setthamalinee.pdf

Shrestha, S., \& Krolak, L. (2015). The potential of community libraries in supporting literate environments and sustaining literacy skills. International Review of Education, 61, (3), 399-418.

Smalley W.A. (1994). Linguistic diversity and national unity: Language ecology in Thailand. Chicago, IL: University of Chicago Press.

Stake, R.E. (1995). The Art of case study research. Thousand Oaks, CA: Sage. (2006). Multiple case study analysis. New York. NY: The Guilford Press.

Stranger-Johannessen, E. (2014). Promoting a reading culture through a rural community library in Uganda. IFLA Journal, 40(2), 92-101.

. Asselin, M., \& Doiron, R. (2015). New perspectives on community library development in Africa. New Library World, 116(1/2), 79 - 93.

Sturges, P. (2014). Donations to Libraries: A Problem in International Cooperation in S. Chakraborty and A. Kumar Das (eds). Collaboration in International and Comparative Librarianship 17-27.

Suchiva, N. (2016). PUBAT: Thais still love books. Retrieved from http://www.bangkok post. com /business /news/1110965/pubat-thais-still-love-books.

Sugiharto, S. (2011, August 24-25). The Myth of non-literate Culture: The Case of Indonesian Children. Paper presented at Thailand Conference on Reading 2011, Bangkok, Thailand.

Sullivan, A., \& Brown, M. (2013). Social inequalities in cognitive scores at age 16: The role of reading. London: Centre for Longitudinal Studies, University of London.

Suwanpitak, S. (2008). Thailand's path to literacy. International Review of Education $54,763-771$. 
Talja, S., Tuominen, K. \& Savolainen, R. (2005). "Isms" in information science: constructivism, collectivism and constructionism. Journal of Documentation, 61(1), $79-101$.

Tambiah, S. (1968). Literacy in a Buddhist village in north-east Thailand in J. Goody (Ed.) Literacy in traditional societies (pp. 86-131). Cambridge: Cambridge University.

Tamrackitkun, K. (2010). Extensive Reading: An empirical study of its effects on EFL Thai students' reading comprehension, reading fluency and attitudes. (Doctoral dissertation University of Salford, United Kingdom). Retrieved from http://usir.salford.ac.uk/11924/

Tan, T. (2011). Children's Publishing in Asia. Retrieved from https://www.publishers weekly.com/pw/by-topic/childrens/childrens-industry-news/article/46447children-s-publishing-in-asia.html

Thai Tribune. (2017). Dr. Prasert pointed out the end of Learning by Memorizing Century and suggested state unlock exam in grade 1 . Retrieved from http://www.thaitribune.org/contents/detail/304?content_id=30619\&rand=151 3302972

The American Heritage New Dictionary of Cultural Literacy. (2016). 3rd ed. Retrieved from http://www.dictionary.com /browse/ socioeconomic-status

The Asia Foundation. (2013). Profile of the protestors: A Survey of pro and antigovernment demonstrators in Bangkok on November 30, 2013. Retrieved from https://asiafoundation.org/resources/pdfs /FinalSurveyReportDecember20.pdf

TK Park. (2008). Thais' reading attitudes and reading behaviours. Bangkok, Thailand: TK Park. (In Thai) - (2009). 20 perspectives to challenge the reading dilemma. Bangkok, Thailand: TK Park. (In Thai)

(2010). Thailand reading situation and reading index 2010: Development of reading index to and factor affecting the reading index 2010. Bangkok, Thailand: TK Park. (In Thai)

-.-. (2015). Collaboration. Retrieved from http://www.tkpark.or.th/tha/page /network_map (2018). Box: Library world, learning world, experience world. Bangkok, Thailand: TK Park. 
Tosakul, R. (2013). Chapter 9 Relatedness: Capitalism and the Sufficiency Economy in rural Northeastern Thailand in P. Liamputtong (Ed.) Contemporary SocioCultural and Political Perspectives in Thailand (pp. 140-153). Heidelberg, Germany: Springer Netherlands.

Trading Economics. (2018). Thailand minimum daily wage 1973-2018. Retrieved from https://tradingeconomics.com/thailand/minimum-wages

Trentacosti, G. (2015). Thailand's transition towards digital publishing: Thai publishers are called to capitalize on smartphone and online reading momentum. Retrieved from http://2seasagency.com/international-publishing-insightsthailand/.

Tunmer, W.E., Chapman, J.W., Greaney, K.T., Prochnow, J.E., \& Arrow, A.W. (2013). Why the New Zealand National Literacy Strategy has failed and what can be done about it: Evidence from the Progress in International Reading Literacy Study (PIRLS) 2011 and Reading Recovery monitoring reports. Australian Journal of Learning Difficulties, 18(2), 139-180.

Taylor, R.S. (1968). Question-negotiation and information seeking in libraries. College \& Research Libraries, 29(3), 178-194.

UNESCO. (2008). Improving the quality of mother tongue-based literacy and learning: Case Studies from Asia, Africa and South America. Bangkok: UNESCO Bangkok. Retrieved from http://unesdoc.unesco.org/images/0017/001777 /177738e.pdf

--... (2014). Reading in the Mobile Era: A Study of mobile reading in developing countries. Retrieved from www.unesco.org/new/en/unesco/themes/icts/m4ed /mobile-reading/reading-in-the-mobile-era/

. (2017). 2017/2018 Global Education Monitoring Report: Thailand highlights. Retrieved from https://bangkok.unesco.org/content/20172018-globaleducation-monitoring-report-thailand-highlights

UNICEF. (2016). Early childhood development. Retrieved from http://www.unicef.org/ thailand/education_14938.html

UNICEF Thailand. (2014). Public Expenditure Tracking Survey (PETS) on the 15-Year Free Education Program: Kingdom of Thailand. Retrieved from https://www.unicef .org/thailand/PETS_ENG_web.pdf

United Kingdom Literacy Association (2008). Teachers as readers: Building communities of readers, 2007-08 Executive Summary. London: United Kingdom Literacy Association.

United Nations. (2002). A World Fit for Children adopted by the UN General Assembly at the 27th Special Session of the UN General Assembly, 10 May 2002, Annex, no. 7 para. 4. 
Vanhaleweyk, G. (2017). GDP per capita and population data for the provinces of Thailand. Retrieved from http://www.thaiwebsites.com/provinces-GDP.asp.

Veinot, T.C., \& Williams, K. (2012). Following the "community" thread from sociology to information behaviour and informatics: Uncovering theoretical continuities and research opportunities. Journal of the American Society for Information Science and Technology, 63(5), 847-864.

Vongsirinavarn, M. \& Pleehajinda, T. (2010). Study on book rotation system in small primary schools. Bangkok: National Research Council of Thailand. (In Thai)

Wallace, D.P., \& Van Fleet, C. (2012). Knowledge into Action: Research and Evaluation in Library and Information Science. Santa Barbara, CA: Libraries Unlimited.

Warr, P. (2007). Long-term economic performance in Thailand. ASEAN Economic Bulletin, 24(1), 138-163.

Wasee, P. (2011). The education system that would heal the nation. Nakorn Pathom, Thailand: Contemplative Education Center, Mahidol University. (In Thai) (2014). Leadership for the future. Nakornprathom, Thailand : Contemplative Education Center, Mahidol University. (In Thai)

Wech-o-sotsakda, C. (2015). Reading culture in produce organic vegetables group at Don Du Village, Kanthararat Sub-district, Katharawichai District, Mahasarakham Province. Journal of library and information science Srinakharinwirot University. $7(2), 14-22$.

Wexelbaum, R. (2016). The Library as safe space. In S. S. Hines and K. M. Crowe (Ed.), The Future of library space (pp. 37-78). Bingley, UK: Emerald.

Wildemuth, M.B. (2009). Applications of social research methods to questions in Information and Library Science. Westport, CT: Libraries Unlimited.

Wimolsittichai, N. (2017). School libraries and their roles in rural Thailand: Perceptions of public primary school principals. (Doctoral dissertation, Queensland University of Technology, Brisbane, Australia). Retrieved from https://eprints. qut.edu.au /view/person/Wimolsittichai,_Nilobon.html

World Bank. (1996). Thailand growth, poverty and income distribution: An Economic report. Bangkok, Thailand: Country Operations Division, East Asia and Pacific Region.

World Bank Group. (2016). Data: Education. Retrieved from http://data.worldbank.org /topic/education

World Economic Forum. (2018). The Global Competitiveness Report 2017-2018. Retrieved from http://www3.weforum.org/docs/GCR2017-2018/05Full Report/ TheGlobalCompetitivenessReport2017\%E2\%80\%932018.pdf 
Wyatt, D. K. (1969). The politics of reform in Thailand: Education in the Reign of King Chulalongkorn. New Haven: Yale University Press.

Yin, R. K. (2014). Case study research: Design and methods. 5th ed. Thousand Oaks, CA: Sage.

Yutisri, P. (2015). The Publishing Industry in Thailand. Publishing Research Quarterly 32(3), 261-265.

Zach, L. (2006). Using a Multiple-case studies design to investigate the informationseeking behavior of Arts administrators. Library Trends, 55(1) 4-21. 


\section{Appendices}

\section{Appendix A}

Information sheet and consent form for participating community libraries 


\title{
Participant Information Sheet for Participating Community Libraries
}

\author{
Research Project Title: Factors Affecting Reading for Pleasure Practices in Community \\ Libraries in Thailand
}

Researcher: $\quad$ Chommanaad Boonaree, School of Information Management, Victoria University of Wellington, New Zealand

As part of the completion of my PhD, I am undertaking a study designed to explore the factors affecting reading for pleasure (RfP) practice in community libraries (CLs) in the Northeast region of Thailand, and how these factors facilitate or hinder RfP practice in the region. The study will support the Thai government's attempts to identify appropriate practices to promote reading behaviour within the region.

For selected CLs, I am inviting three groups of people to participate in this research. They are: 1) educators/local reading promoters, 2) local leaders/CL committee, and 3) CL staff/ volunteers. In each library, data will be collected in the following manner:

1. Semi-structured interviews. I will interview individuals who are involved with the practice of RfP in the community. Participants will be asked to take part in an hour-long interview. Permission will be asked to audio record the interview, and a transcript of the interview will be sent to participants for checking.

2. Observation. I will observe the RfP practice in the library by conducting library visits and attending relevant library activities. Photographs will be taken and used for data analysis but will not be used in my thesis nor in any published reports. A Consent Form for Observation and an Observation Participant Consent Form for Librarians will be completed by the authorised persons responsible for library operation and activities being observed.

In addition, I will request information that is directly related to RfP practice such as statistical records of books borrowed and relevant management documents (i.e. annual plan, service policy, and acquisition policy).

Participation is voluntary, and the name of your library will not be identified in any written report produced as a result of this research including possible publication in academic conferences and journals. All material collected will be kept confidential, and will be viewed only by myself and my supervisors, Professor Anne Goulding and Dr. Dan Dorner. The thesis will be submitted for examining to the School of Information Management, and subsequently deposited in the University Library. Should any library wish to withdraw from the project, they may do so until 30th March 2016 and the data 
collected from them up to that point will be destroyed. All data collected from libraries will be destroyed within 5 years after the completion of the project.

If you have any questions or would like to receive further information about the project, please contact me at tara.boonaree@vuw.ac.nz or telephone 089444 4887, or you may contact my supervisors Professor Anne Goulding and Dr. Dan Dorner at anne.goulding@vuw.ac.nz and dan.dorner@vuw.ac.nz or telephone 0016444635309.

The research has been approved by Victoria University of Wellington Human Ethics Committee. Should you have any concerns about this study, please contact Associate Professor Susan Corbett at susan.corbett@vuw.ac.nz, or telephone 0016444635480.

Chommanaad Boonaree 


\title{
Letter for Library Access
}

\author{
Chommanaad Boonaree \\ School of Information Management \\ Victoria University of Wellington, New Zealand
}

Date:

To: (Name of library)

Re: Permission to gain access to the library and its staff

As part of the completion of $\mathrm{my} \mathrm{PhD}, \mathrm{I}$ am undertaking a study designed to explore the factors affecting reading for pleasure (RfP) practice in community libraries (CLs) in the Northeast region of Thailand, and how these factors facilitate or hinder RfP practice in the region. The study will support the Thai government's attempts to identify appropriate practices to promote reading behaviour within the region.

For selected CLs, I am inviting three groups of people to participate in this research. They are: 1) educators/local reading promoters, 2) local leaders/CL committee, and 3) CLs staff/ volunteers. In each library, data will be collected mainly by semi-structured interviews and observation. Photographs will be taken and used for data analysis but will not be used in my thesis nor in any published reports. In addition, I will request information that is directly related to RfP practice such as statistical records of books borrowed and relevant management documents (i.e. annual plan, service policy, and acquisition policy).

I wish to seek permission from you to gain access to (name of the library) and its staff. Details of my study can be found in the attached Participant Information Sheet for Participating Community Libraries.

\section{Best regards}

Chommanaad Boonaree 


\title{
Appendix B
}

\section{Information sheet and consent form for individual interviewees}

\author{
B 1 Participant information sheet
}

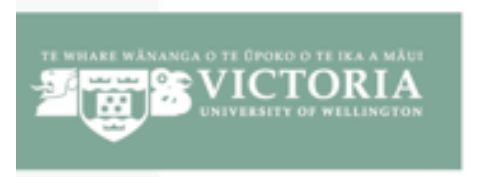

SCHOOL OF INFORMATION MANAGEMENT

TE KURA TLAKI, WHAKCAWHITI KÖRERO

LEVEL 5, RUTHERFORD HOUSE, PIPITEA CAMPUS, 23 LAMBTON QUAY, WELLINGTON

PO Box 600, Wellington 6140, New Zealand

Phone + 64-44463 5103Fax +64-4-463 5446Emailsim Qvuw.ac.nzWebaitewwr.victoria. ac.nz/sim

\section{Participant Information Sheet}

Research Project Title: Factors Affecting Reading for Pleasure Practices in Community Libraries in Thailand

Researcher: $\quad$ Chommanaad Boonaree, School of Information Management, Victoria University of Wellington

As part of the completion of my PhD, I am undertaking a study designed to explore the factors affecting reading for pleasure (RfP) practice in community libraries (CLs) in the Northeast region of Thailand, and how these factors facilitate or hinder RfP practice in the region. The study will support the Thai government's attempts to identify appropriate practices to promote reading behaviour within the region.

I am inviting you to participate in this research. In first stage of the study I aim to interview individuals who are involved with the practice of RfP in the community. Participants will be asked to take part in an hour-long interview. Permission will be asked to audio record the interview, and a transcript of the interview will be sent to participants for checking.

Participation is voluntary, and you will not be identified personally in any written report produced as a result of this research including possible publication in academic conferences and journals. All material collected will be kept confidential, and will be viewed only by myself and my supervisors, Professor Anne Goulding and Dr. Dan Dorner. The thesis will be submitted for examining to the School of Information Management, and subsequently deposited in the University Library. Should any participants wish to withdraw from the project, they may do so until 30th March 2016 and the data collected from them up to that point will be destroyed. All data collected from participants will be destroyed within 5 years after the completion of the project.

If you have any questions or would like to receive further information about the project, please contact me at tara.boonaree@vuw.ac.nz or telephone 0894444887 , or you may contact my supervisor Professor Anne Goulding and Dr. Dan Dorner at anne.goulding@vuw.ac.nz and dan.dorner@vuw.ac.nz or telephone 001644463 5309.The research has been approved by Victoria University of Wellington Human Ethics Committee. Should you have any concerns about this study, please contact Associate Professor Susan Corbett at susan.corbett@vuw.ac.nz, or telephone 0016444635480. 


\title{
Consent Form for Interview
}

\author{
Research Project Title: Factors Affecting Reading for Pleasure Practices in \\ Community Libraries in Thailand \\ Researcher: \\ Chommanaad Boonaree, School of Information Management, \\ Victoria University of Wellington, New Zealand
}

I have been given and have understood an explanation of this research project. I have had an opportunity to ask questions and have them answered to my satisfaction.

I understand that I may withdraw myself (or any information I have provided) from this project, without having to give reasons, by e-mailing tara.boonaree@vuw.ac.nz or telephone 0894444887 by $30^{\text {th }}$ March 2016.

I understand that any information I provide will be kept confidential to the researcher and their supervisor, the published results will not use my name, and that no opinions will be attributed to me in any way that will identify me or my organisation, though I understand that in some cases readers might be able to identify related government organizations from the context of this study.

I understand that the data I provide will not be used for any other purpose or released to others.

I understand that, if this interview is audio recorded, the recording and transcripts of the interviews will be erased within 5 years after the conclusion of the project. Furthermore, I will have an opportunity to check the transcripts of the interview.

Please indicate (by ticking the boxes below) which of the following apply:

I would like to receive a summary of the results of this research when it is completed.

I agree to this interview being audio recorded.

I agree to the interviewer taking notes.

Signed:

Name of participant:

Organization's name and department:

Name of library:

Date: 


\section{Appendix C}

\section{Individual Interview Protocol}

\section{Interview Protocol}

\section{Resources for interviews}

1.1 Recorder and extra batteries for the recorder

1.2 Pens and notebooks

1.3 Copy of signed interview consent form

1.4 Copies of letter of information, interview consent form to be provided to interviewees in case the researcher has not received signed consent form.

1.5 A note taker (research assistant), in case that interviewees do not agree to be recorded.

\section{Interview schedule}

\subsection{Date/Time}

2.2 Name of interviewee

2.3 Organisation and position

2.4 Contact details of interviewee

\section{Procedure of interviews}

\subsection{Advance Preparation}

a. Contact the interviewees and schedule the time for interviews

b. Send the information sheets and consent forms to them

\subsection{Introduction}
a. Introduce myself
b. Explain the purpose of the study and the purpose of the interview
c. Explain why the participant has been invited and why he or she is important to the study
d. Explain the term "reading for pleasure (RfP)"

\subsection{Interviewing}
a. Start with their working experience in community libraries (CLs)
b. Ask about their perspectives on the function of $\mathrm{CLS}$
c. Ask about their personal experience in reading promotion activities
d. Ask about their understanding of reading for pleasure (RfP) concepts and practices
e. Ask for their opinions about factors (enablers and barriers) affecting RfP practice in $\mathrm{CLS}$
f. Based on the flow of discussion, ask follow-up questions
g. Ask for their perspectives on factors in the preliminary model which they have not yet mentioned
h. Do a final check of the list (below) of Areas for questioning to ensure all areas have been discussed.

\subsection{Closing}

a. Ask the participant if there are any additional ideas

b. Thank the participant for participation and ask for their future help when needed 


\subsection{Preliminary Review}

Review notes and do preliminary coding to formulate lessons learned to be applied in the next interviews.

\section{Areas for Questioning:}

4.1 Working experience in community libraries (CLs)

4.2 Perspectives on the function of CLs

4.3 Personal experience in reading promotion activities

4.4 Understanding of reading for pleasure (RfP) concepts and practices

4.5 Factors affecting RfP in CLs (from preliminary model)

a. Government policy and policy implementation- e.g. literacy policy, education policy

b. Language environment - e.g. mother-tongue language

c. Reading culture - e.g. reading policy, cooperation,

d. Social and cultural elements - e.g. social structure, collectivism, learning tradition

e. CLs management elements - e.g. library materials, staffing 


\section{Appendix D}

\section{Themes for gathering evidence}

\section{THEMES FOR GATHERING EVIDENCE}

Themes

1. LITERACY

1.1 Family Literacy

1.2 Thai Literacy Policy

1.3 Isan Language

1.4 Reading in a Second Language

2. DEVELOPMENT OF READING CULTURE

2.1 The Government Reading Policy

2.2 Cooperation by Organizations in Society

2.3 Parents and Teachers

2.4 Reading Atmosphere

2.5 Sharing Reading

3. SOCIAL AND CULTURAL ELEMENTS

3.1 Vertical Social Structure

3.2 Collectivist Society

3.3 Normative Culture

3.4 Learning Tradition

3.5 Isan Identity

4. COMMUNITY LIBRARY MANAGEMENT ELEMENTS

4.1 Reading Materials

4.2 Staffing

4.3 Library Activities/Services 


\title{
Appendix E
}

\section{Information sheets and consent forms for observation}

\author{
E 1 Observation participant information sheet for librarians
}

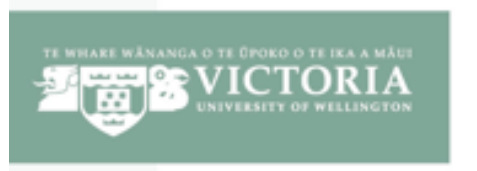

SCHOOL OF INFORMATION MANAGEMENT

TE KURA TLAKI, WHAKAWHITI KÖRERO

LEVEL 5, RUTHERFORD HOUSE, PIPITEA CAMPUS, 23 LAMBTON QUAY, WELLINGTON

PO Box 600, Wellington 6140, New Zealand

Phone + 64-4-463 5103Fax +64-4-463 5446Emailsim Qvuw.ac.nzWebaitewwar.victoria.ac.nz/sim

(E1)

\section{Observation Participant Information Sheet for Librarians}

\author{
Research Project Title: Factors Affecting Reading for Pleasure Practices in Community \\ Libraries in Thailand \\ Researcher: $\quad$ Chommanaad Boonaree, School of Information Management, \\ Victoria University of Wellington, New Zealand
}

As part of the completion of my PhD, I am undertaking a study designed to explore the factors affecting reading for pleasure (RfP) practice in community libraries (CLs) in the Northeast region of Thailand, and how these factors facilitate or hinder RfP practice in the region. The study will support the Thai government's attempts to identify appropriate practices to promote reading behaviour within the region. Victoria University requires, and has granted, approval from the School's Human Ethics Committee. Should you have any concerns about this study, please contact Associate Professor Susan Corbett at susan.corbett@vuw.ac.nz, or telephone 0016444635480.

This is an invitation to participate in the study. I will observe library activities today that are relevant to RfP practice and will take notes of what the librarian does and says and how the attendees react. Photographs will be taken and used for data analysis but will not be included in any published reports. Participation is voluntary, and neither you nor any attendees will be identified personally in any written report produced as a result of this research, including possible publication in academic conferences and journals. All material collected will be kept confidential, and will be viewed only by myself and my supervisors: Professor Anne Goulding and Dr. Dan Dorner. School of Information Management, Victoria University of Wellington.

Your involvement in this study is voluntary and you may withdraw your participation from the study up to 30th March 2016 and withdraw any data that has been gathered from you to that point.

All data collected from participants will be destroyed within 5 years after the completion of the project.

If you have any questions or would like to receive further information about the project, please contact me at tara.boonaree@vuw.ac.nz or telephone 089444 4887, or you may contact my supervisor Professor Anne Goulding and Dr. Dan Dorner at anne.goulding @vuw.ac.nz and dan.dorner@vuw.ac.nz or telephone 00164 4463 5309. 


\section{Observation Participant Consent Form for Librarians}

Research Project Title: Factors Affecting Reading for Pleasure Practices in Community Libraries in Thailand

Researcher: Chommanaad Boonaree, PhD Student, School of Information Management, Victoria University of Wellington, NZ.

I have been given and have understood an explanation of this research project. I have had an opportunity to ask questions and have them answered to my satisfaction.

I understand that I may withdraw myself (or any information I have provided) from this project, without having to give reasons, by e-mailing tara.boonaree@vuw.ac.nz by 30th March 2016 or by calling 0894444887 .

I understand that any notes taken in the session will be kept confidential to the researcher and her supervisors, the published results will not use any names, and that no actions or words will be attributed in any way that will identify me.

I understand that the data I provide will not be used for any other purpose or released to others.

I understand that, the notes and photos from the observation session will be erased within 5 years after the conclusion of the project. Furthermore, I will have an opportunity to receive a summary of the results of the research by emailing the researcher.

Signed:

Name of participant:

Name of the library:

Date: 


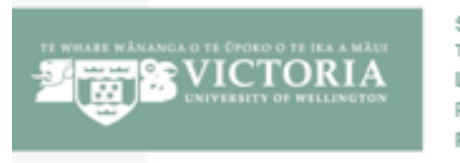

\title{
Observation Participant Information Sheet for Parents/Caregivers
}

\author{
Research Project Title: Factors Affecting Reading for Pleasure Practices in Community \\ Libraries in Thailand \\ Researcher: $\quad$ Chommanaad Boonaree, School of Information Management, \\ Victoria University of Wellington
}

\begin{abstract}
As part of the completion of my PhD, I am undertaking a study designed to explore the factors affecting reading for pleasure (RfP) practice in community libraries (CLs) in the Northeast region of Thailand, and how these factors facilitate or hinder RfP practice in the region. The study will support the Thai government's attempts to identify appropriate practices to promote reading behaviour within the region. Victoria University requires, and has granted, approval from the School's Human Ethics Committee. Should you have any concerns about this study, please contact Associate Professor Susan Corbett at susan.corbett@vuw.ac.nz, or telephone 0016444635480.
\end{abstract}

This is an invitation to participate in the study. I will observe the library activities today that are related to RfP practice and will take notes of what the librarian does and says and how the users react. Photographs will be taken and used for data analysis but will not be used in my thesis nor in any published reports.

Your involvement in this study is voluntary and you may withdraw yourself and any children who are with you from the study before the activity begins. Neither you nor any of the children attending the activities will be identified personally in any written report produced as a result of this research, including possible publication in academic conferences and journals. All material collected will be kept confidential, and will be viewed only by myself and my supervisors: Professor Anne Goulding and Dr. Dan Dorner, School of Information Management, Victoria University of Wellington.

All data collected from participants will be destroyed within 5 years after the completion of the project.

If you have any questions or would like to receive further information about the project, please contact me at tara.boonaree@vuw.ac.nz or telephone 0894444887 , or you may contact my supervisor Professor Anne Goulding and Dr. Dan Dorner at anne.goulding @vuw.ac.nz and dan.dorner@vuw.ac.nz or telephone 0016444635309.

Chommanaad Boonaree 


\section{Factors Affecting Reading for Pleasure Practices in Community} Libraries in Thailand

\section{Observation Verbal Consent Script}

Hello, my name is Chommanaad Boonaree and I am a PhD student in the School of Information Management, Victoria University of Wellington, New Zealand

I'm currently involved in a research study looking at reading for pleasure (RfP) practice in community libraries (CLs) in the Northeast region of Thailand. Here is a sheet which gives you more information about the study [hand out Participant Information Sheet -E3].

Today, I would like your permission to sit in the library to observe how the librarian and children engage in reading activities. I will be taking notes throughout my 1-hour visit, observing how the librarian and children engage in reading activities. Photographs will be taken and used for data analysis but not be used in published reports.

Being in this study is optional, and you can tell me if you want to stop being in the study at the beginning of the activity.

Do you have any questions about the study?

Is there anybody who is not happy about me observing the session today?

If you have questions about this study in the future, you can contact me at tara.boonaree@vuw.ac.nz by 30th March 2016 or call 0894444887. 


\section{Observation Witness Consent Form}

STUDY TITLE: Factors Affecting Reading for Pleasure Practices in Community Libraries in Thailand

INVESTIGATOR: Chommanaad Boonaree, School of Information Management, Victoria University of Wellington, New Zealand

I confirm that the researcher has explained the elements of informed consent to the participants.

The subjects know that their participation is voluntary, and that they can withdraw at the beginning of the activity. The purpose of the research has been explained and the procedures have been outlined. The participants understand issues of confidentiality.

Witness Name:

Witness Signature: 


\title{
Appendix F
}

\section{Research assistant and research transcriber confidentiality agreements}

F 1 Research assistant confidentiality agreement

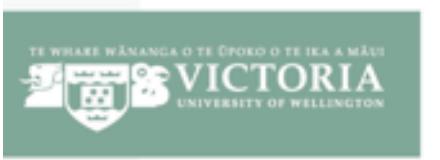

SCHOOL OF INFORMATION MANAGEMENT

TE KURA TLAKI, WHAKCAWHITI KÖRERO

LEVEL 5, RUTHERFORD HOUSE, PIPITEA CAMPUS, 23 LAMBTON QUAY, WEUUNGTON

PO Bax 600, Welington 6140, New Zealand

Phone + 64-4-463 5103Fax +64-4-463 5446Emailsimgvuw. ac.nzWebsitenww. victoria. ac.nz/sim

\author{
Note Taker Confidentiality Agreement \\ Research project: \\ Factors Affecting Reading for Pleasure Practices in Community \\ Libraries in Thailand \\ Researchers: \\ Chommanaad Boonaree \\ School of Information Management, Victoria University of Wellington
}

I have read the information sheets that have been sent to participants of this research project.

I agree to treat the names of the participants and their responses as confidential.

All electronic information will be kept in a password-protected file and all written material will be kept in a locked file

Name:

Signature:

Date: 


\title{
Transcriber Confidentiality Agreement
}

Research project:

Factors Affecting Reading for Pleasure Practices in Community Libraries in Thailand

\section{Researchers:}

Chommanaad Boonaree

School of Information Management, Victoria University of Wellington

\begin{abstract}
$\square$ I have read the information sheets that have been sent to participants of this research project.

$\square$ I agree to treat the names of the participants and their responses as confidential.

$\square$ All electronic information will be kept in a password-protected file and all written material will be kept in a locked file.
\end{abstract}

Name:

Signature:

Date: 


\section{Translator Confidentiality Agreement}

Research project:

Factors Affecting Reading for Pleasure Practices in Community Libraries in Thailand

\section{Researchers:}

Chommanaad Boonaree

School of Information Management, Victoria University of Wellington

\footnotetext{
$\square$ I have read the information sheets that have been sent to participants of this research project.

$\square$ I agree to treat the names of the participants and their responses as confidential.

$\square$ All electronic information will be kept in a password-protected file and all written material will be kept in a locked file.
}

Name:

Signature:

Date: 